\title{
Risk attitude : preference models and applications to bargaining
}

Citation for published version (APA):

Köbberling, V. (2003). Risk attitude : preference models and applications to bargaining. [Doctoral Thesis, Maastricht University]. Universiteit Maastricht. https://doi.org/10.26481/dis.20030424vk

Document status and date:

Published: 01/01/2003

DOI:

10.26481/dis.20030424vk

Document Version:

Publisher's PDF, also known as Version of record

\section{Please check the document version of this publication:}

- A submitted manuscript is the version of the article upon submission and before peer-review. There can be important differences between the submitted version and the official published version of record.

People interested in the research are advised to contact the author for the final version of the publication, or visit the DOI to the publisher's website.

- The final author version and the galley proof are versions of the publication after peer review.

- The final published version features the final layout of the paper including the volume, issue and page numbers.

Link to publication

\footnotetext{
General rights rights.

- You may freely distribute the URL identifying the publication in the public portal. please follow below link for the End User Agreement:

www.umlib.nl/taverne-license

Take down policy

If you believe that this document breaches copyright please contact us at:

repository@maastrichtuniversity.nl

providing details and we will investigate your claim.
}

Copyright and moral rights for the publications made accessible in the public portal are retained by the authors and/or other copyright owners and it is a condition of accessing publications that users recognise and abide by the legal requirements associated with these

- Users may download and print one copy of any publication from the public portal for the purpose of private study or research.

- You may not further distribute the material or use it for any profit-making activity or commercial gain

If the publication is distributed under the terms of Article $25 \mathrm{fa}$ of the Dutch Copyright Act, indicated by the "Taverne" license above, 


\section{Risk Attitude:}

Preference Models and Applications to Bargaining 


\section{Risk Attitude:}

\section{Preference Models and Applications to Bargaining}

\section{PROEFSCHRIFT}

ter verkrijging van de graad van doctor aan de Universiteit Maastricht, op gezag van de Rector Magnificus, Prof. dr. A.C. Nieuwenhuijzen Kruseman, volgens het besluit van het College van Decanen, in het openbaar te verdedigen op donderdag 24 april 2003 om 16.00 uur

door

Veronika Köbberling 


\section{Promotores:}

Prof. dr. H.J.M. Peters

Prof. dr. P.P. Wakker

Beoordelingscommissie:

Prof. dr. J.J. Herings (voorzitter)

Dr. H. Bleichrodt (Erasmus Universiteit Rotterdam)

Prof. dr. G. Kirchsteiger

NWO Project 425-11-003-P 


\section{Contents}

Preface

1 Introduction

2 Strength of Preference and Cardinal Utility 5

2.1 Introduction . . . . . . . . . . . . . . . . . . 5

2.2 Preference Foundations . . . . . . . . . . . . . . . . . 7

2.3 Generalizations . . . . . . . . . . . . . . . . .

2.4 Related Literature $\ldots \ldots \ldots \ldots$

2.5 Conclusion . . . . . . . . . . . . . . . . . 15

2.6 Appendix A: Proofs . . . . . . . . . . . . . . . . 16

2.7 Appendix B: Note on "Karni \& Safra (1998)" . . . . . . . . . . 24

3 A Tool for Qualitatively Testing, Quantitatiwely Measuring, and Normatively Justifying Expected Utility 27

3.1 Introduction . . . . . . . . . . . . . . . 27

3.2 The Intuition Underlying our Condition $\ldots \ldots \ldots \ldots$

3.3 Discussion . . . . . . . . . . . . . . . . . . . 35

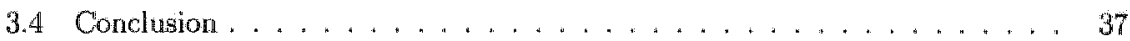

4 Preference Foundations for Nonexpected Utility: A Generalized and Simplifled Technique $\quad 39$

4.1 Introduction . . . . . . . . . . . . . . . . . . . 39

4.2 Basic Definitions of Decisiom under Uncertainty, and their Restrictions to Subsets of the Domain ....................... 41

4.3 A General Tool for Axiomatizing Models . . . . . . . . . . . . . . . 42 
4.4 Applications of our Technique . . . . . . . . . . . . 44

4.4.1 The Algebraic Approach for Subjective Expected Utility . . . . . 44

4.4.2 The Algebraic Approach for Chaquet Expected Utility . . . . . . 47

4.4.3 The Topological Approach for Prospect Theory . . . . . . . . 49

4.5 Further Applications . . . . . . . . . . . . . . . . . . 52

4.5 .1 Infinite State Spaces $\ldots \ldots \ldots \ldots \ldots \ldots . \ldots \ldots$

4.5 .2 Restriction to Two-Outcome Acts . . . . . . . . . . 53

4.5 .3 Decision under Risk. . . . . . . . . . . . . . . . . 53

4.5 .4 Vtility Curvature . . . . . . . . . . . . . . . . 54

4.6 A Review of Axiomatizations of Nonexpected Utility $\ldots \ldots \ldots \ldots 5$

4.7 Conclusion. . . . . . . . . . . . . . . . . . . . . 58

4.8 Appendix A: The Generality of our Approach . . . . . . . . . 58

4.9 Appendix B: Preference-Tradeoff Consistency, and other Implications of Tradeoff Consistency . . . . . . . . . . . . . . . . . . 62

4.10 Appendix $\mathrm{C}$ Further Proofs . . . . . . . . . . . . . . . 70

5 An Index of Loss Aversion $\quad \mathbf{7 5}$

5.1 Intraduction . . . . . . . . . . . . . . 75

5.2 Prospect Theory . . . . . . . . . . . . . . . . 77

5.3 An Index of Loss Aversion . . . . . . . . . . . . . . . . 78

5.4 Comparative Loss Aversion . . . . . . . . . . . . . . . . . 79

5.5 Empirical Findings and Suggestions . . . . . . . . . . . . 81

5.6 Alternative Definitions of Loss Aversion . . . . . . . . . . . . . 83

5.7 Concluding Remarks . . . . . . . . . . . . . . . . . 85

5.8 Appendix A: Implications for Parametric Families of Utility . . . . . . 85

5.9 Appendix B: Proofs . . . . . . . . . . . . . . . . . . . . . . . 87

6 Comparative Loss Aversion in Prospect Theory 89

6,1 Introduction . . . . . . . . . . . . . . . . . . . 89

6.2 Prospect Theory for General Outcomes . . . . . . . . . . . . . . 90

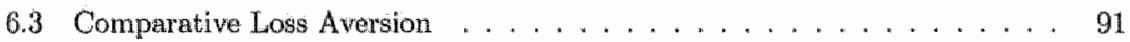

6.4 Conclusion. . . . . . . . . . . . . . . . . . . . . . 93

6.5 Appendix . . . . . . . . . . . . . . . . . 93 
7 The Effect of Decision Weights in Bargaining Problems 97

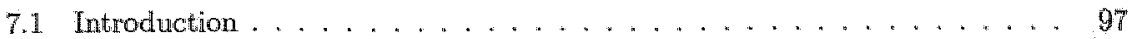

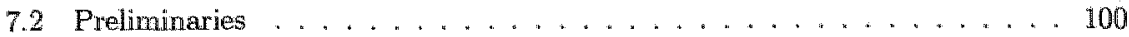

7.2 .1 Rank-dependent Utility . . . . . . . . . . . . . 100

7.2 Comparatiwe Risk Aversion . . . . . . . . . . . . . . . . . 100

7.2 .3 Bargaining Problems and Solutions . . . . . . . . . . . 101

7.2 .4 Feasible Sets of Bargaining Problens . . . . . . . . . . . . . 102

7.2 .5 The Kalat-Smorodinsky Bargaining Solution . . . . . . . . . . 103

7.3 Sensitivity of the KS Bargaining Solution to Risk . . . . . . . . . . . 104

7.3 .1 Utility Risk Aversion . . . . . . . . . . . . . . . 104

7.3 .2 Probabilistic Risk Aversion .................. 104

7.3 .3 Discussion and Strategic Implications . . . . . . . . . . . 105

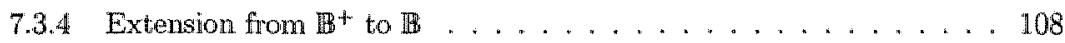

7.4. An Axiomatization of the Kalai-Smonodinsky Bargaining Solution . . . . 109

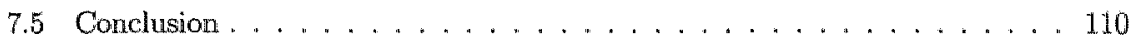

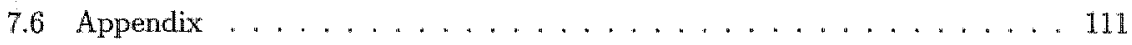

8 Loss Aversion in Bargaining Problems $\quad 121$.

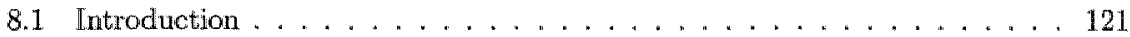

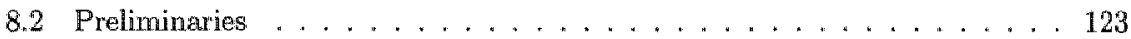

8.2.1 Prospect Theory and Loss Aversion . . . . . . . . . . . . . . . . . 123

8.2.2 Bargaining Problems and Solutions . . . . . . . . . . . . 124

8.2.3 The Kalai-Smorodinsky Bargaining Solution . . . . . . . . . . 125

8.2 .4 The Kalai-Rosenthal Bargaining Solution . . . . . . . . . . . 126

8.3 Loss Awersion in Bargaining . . . . . . . . . . . . . . . . . 126

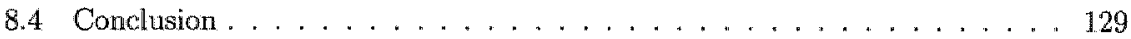

9 An Empirical Test of Numeraire Illusion by Comparing Rukk Attitudes Before and After the Introduction of the Euro 131

9.1 Introduction . . . . . . . . . . . . . . . . . . 132

9.2 Method . . . . . . . . . . . . . . . . . . . . . . . . . . . . . . . . . . . . . . . . .

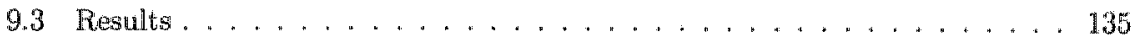

9.4 Conclusion . . . . . . . . . . . . . . . . . . . 137

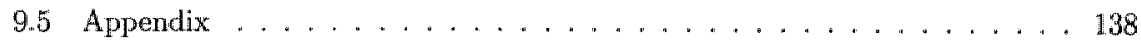




\section{Preface}

About four years ago, I started my Ph.D.-research under the supervision of Prof. Dr. Hans Peters and Prof. Dr. Peter P. Wakker. Most of the results in this thesis were first formulated in joint papers, written within the last four years. I am truly grateful to Hans Peters and Peter P. Wakker, who both advised and supported me in a dedicated and very friendly manner.

Also, I would like to thank my family and friends for moral (and financial) support, as well as my colleagues at the Department of Quantitative Economics and beyond for their help, their suggestions and the pleasant working atmosphere.

Finally, I would like to thank the Dutch Science Foundation, NWO for financial support under grant 425-11-003-P. NWO also financed a two month visiting scholarship at the Kellogg School of Management, Northwestern University, USA, in spring 2002. 



\section{Chapter 1}

\section{Introduction}

This thesis consists of four different parts. The first part (Chapters 2, 3 \& 4) provides axiomatic foundations for cardinal utility (Chapter 2), expected utility (Chapter 3) and nonexpected utility (Chapter 4) based on preference conditions. The benefit from axiomatizations is threefold.

First, the used preference conditions represent a link between observable (or introspective) preference judgements and the model under consideration. The conditions improve our understanding of the different ingredients (e.g. the utility function) and help us to see the consequences and limitations of the model. Second, the characterizing preference conditions can be used for justifying or criticizing the model under consideration. If all the conditions are intuitively plausible and normatively desirable, this must also hold for the model. The preference conditions might convince an individual who's preferences do not fit the model, but who considers the conditions as desirable, to change some of the preferences in order to make better decisions. Third, the conditions can be used to test the validity of the model empirically. The model itself is not directly observable, but the characterizing preference conditions can be verified. If it turns out that a model is empirically not valid, the violated conditions can help developing a new model that better accommodates the observed data.

It is desirable that the conditions used to characterize a model, are as simple and general as possible. By providing simpler and more general preference foundations for difference representations, Chapter 2 shows that cardinal utility is easier to obtain than traditionally thought. The chapter unifies all earlier derivations by showing that they can be obtained as direct corollaries. Chapter 3 introduces a new preference condition based on comparisons of 
tradeoffs that can be used for characterizing expected utility. The new condition simplifies and generalizes previously-published tradeoff conditions, and is, at the same time, better suited for empirical measurements of utility than its predecessors, and accordingly easier to test. Chapter 4 generalizes the methods of Chapter 3 and extends them to rankdependent utility and prospect theory. Whereas Chapter 3 is particularly addressed to readers without a strong mathematical background, Chapter 4 is the theoretical, more advanced, and more general counterpart.

Rank-dependent utility and prospect theory are both generalizations of expected utility that can better incorporate certain psychological aspects of risk attitude. The risk attitude of an agent can be determined by analyzing how the agent values a lottery in comparison to the sure monetary amount that is equal to the expected value of the lottery. The agent is risk awerse if the sure amount is preferred, risk seeking if the lottery is preferred, and risk neutral in the case of indifference. Usually than risk seeking. In expected utility, risk attitude is modeled through utility curvature alone. The utility function is usually concave, which reflects decreasing marginal utility and implies owerall risk aversion.

In rank-dependent utility, risk attitude can be modeled through an additional component besides utility curvature, namely, through nonlinear weighting of probabilities. Rank-dlependent utility can, for instance, accommodate the prevalent observed behavior that agents overestimate small probabilities and underestimate high probabilities, which results in a mixture of risk averse and risk seeking behavior. Therefore, rank-dependent utility can explain, at the same time, gambling (agents pay more than the expected gain to participate in a lottery with a low chance of winning a high amount) and insurance (the same agents pay more than the expected loss to insure against a high loss that does not occur with a very high probability).

Prospect theory, like rank-dependent utility, allows for nomlinear probability weighting, but in addition it takes into account that agents often focus on the status quo or on a reference point, which further affects their risk attitude. Outcomes are rated relative to the reference point, and are gains or losses. As a consequence, loss aversion, a third component of observed risk aversion, can be modeled in prospect theory.

Loss aversion is the topic of the second part of the thesis (Chapters 5 \& 6). It reflects the observed behavior that agents are more sensitive to losses than to gains, resulting in an overweighting of losses relative to comparable gains. Many empirical studies have 
confirmed loss aversion and have suggested that it is an important component of observed risk aversion. However, little theory on loss aversion has been developed so far. Chapter 5 proposes a theoretically founded index of loss aversion to govern the exchange rate between gain and Ioss utility units if outcomes are monetary. This proposal leads to a clesar decomposition of risk attitude into three distinct components: basic utility, probability weighting, and loss aversion. For monetary outcomes the proposed loss aversion index is a natural idea, but if outcomes are nonmonetary (e.g. health states) the definition of a loss aversion index is problematic. This is discussed in Chapter 6. Nevertheless, also in those cases it is possible to obtain relative comparisons of loss aversion.

The third part of the thesis (Chapters $7 \& 8$ ) applies rank-dependent utility and prospect theory to bargaining game theory, and examines the consequences of the three components of risk attitude in bargaining. Game theory models situations where two or more agents with conflicting interest interact. Bargaining is a typical example. The gametheoretic setup abstracts and simplifies real world problems, so that the problems can be analyzed through mathematical tools. The idea is that the mathematical results for the simplified problems give indications for the more complex real world problens.

If two agents bargain, they have certain possibilities to agree on. These possibilities usually comprise uncertainties, so that the agents often do not know the final consequence of their agreement. The risk attitude of the bargainers is likely to influence the result of a bargaining process. Hence, it is a natural idea to analyze the impact of risk attitude. To do so, a simplified mathematical model of a bargaining problem is considered, as follows. A bargaining game problem is described by a set of potential sure outcomes, including a disagreement outcome. The bargainers try to reach agreement on one of the outcomes or on a lottery between some outcomes. If they fail, the disagreement outcome results. The bargainers are assumed to have preferences according to rank-dependent utility (Chapter 7 ) or prospect theory (Chapter 8 ). Different solution concepts to bargaining game problens have been suggested in the literature. Chapters $7 \& 8$ examine the stability of some well-known solution concepts if the risk attitude of one of the bargainers changes. The three components of risk attitude are considered separately (Chapter 7: utility curvature and probability weighting; Chapter 8: loss aversion). It turns out that the components, surprisingly, have partly opposite consequences.

For models and theories that concern psychological aspects of decision making, it is irmportant that they can be tested empirically. Chapter 9 , the last part of the thesis, deals 
with an empirical experiment on risk attitude. The chapter describes a simple experiment that can be used to test whether people, when evaluating money, think in terms of numbers instead of in terms of real values. It is examined whether numbers or real values have more influence on the risk attitude. In the experiment, risk attitudes are compared before and after the introduction of the Euro. The change of currency in Europe gave the unidque opportunity to observe changes in risk attitude caused by changing numbers separattely from that caused by changing real values.

All chapters in the thesis are self-contained. As a consequence, overlaps could nott be avoided. The results of the chapters were first formulated in different research papeers, joint work in part with Peter P. Wakker or Hans Peters. 


\section{Chapter 2}

\section{Strength of Preference and Cardinal}

\section{Utility}

By providing simpler and more general preference foundations for difference representam tions, this chapter shows that cardinal utility is easier to obtain than traditionally thought. The chapter unifies all earlier derivations by showing that they can be obtained from the present chapter as direct corollaries. Furthermore, the results show how to test cardinality empirically. ${ }^{1}$

\subsection{Introduction}

Before the ordinal revolution of the 1930 s, cardinal utility was a central, but not very well founded concept in economics. Logical positivism, which became the dominant scientific and academic approach at the end of the 19th/ beginning of the 20th century, also became influential in economic theory. Only empirically testable claims were accepted, non-testable theories were disapproved. In econornics, the basic idea became to base utility solely on observable choice. From observable choice, only ordinal utility can be derived. Preference intensities or marginal utilities are not directly observable, and therefore, cardinal utility had to be given up. The ordinal view was promoted by, for instance, Hicks \& Allen (1934) and is since then dominant in economics. The question whether utility is cardinal

\footnotetext{
The anthor thalaks particularly Peter $P$. Wakker for helpful comments and sughegtions. Also, the

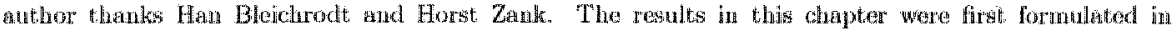
(Kolbbaring, 2002k).
} 
or ordinal has been debated ever since. Cardinal utility is mostly considered to be an outdated concept. Onlly within specific contexts such as decision making under risk (van Neumamn \& Morgenstern, 1944), utilitarian welfare evaluations (Harsamyi, 1955), casebased decision theory (Gilboa, Schmeidler, \& Wakker, 2002), or discounted utilities: for intertemporal evaluations (Samuelson, 1937), cardinal utility is usually accepted.

Preference differences play a key role in obtaining cardinal utility. This means that an individual is not only able to decide which of available commodity bundles is the most. preferred, but also, the individual is able to compare the improvement between a first and a second commodity bundle to the improwement between a third and a fourth commodity bundle. Such comparable preference differences, accompanied by suitable axioms, are sufficient to give cardinal utility. In economics, Pareto (1906) and. Frisch (1926) introduced the idea of comparable preference differences.

An important paper on ordinal versus cardinal utility was Alt (1936). It is the first paper that shows in a mathematically sound way how preference differences, if accepted as primitives, imply cardinal utility. Alt did not advocate either ordinal or cardinal utility, but he argued that relatively simple assumptions that were, surprisingly, also accepted and even used by some ordinalists, are sufficient to imply cardinal utility.

Alt stated that the crucial question is whether comparisons between preference differences can be obtamed from introspection and experience. A positive answer would stupport the cardinal view, a negetive answer the ordinal view. More than forty years later, Sarin (1982) noted: "... Unless the conceptual problem of the meaning and measurement of strength of preference is satisfactorily resolved, this theory will only see limited commercial application." Psychologist are usually less reluctant than economists to permit introspection. Farquhar \& Keller (1989), among others, suggested positive answers to the problem of measuring strength of preferences, and discussed different meastrement procedures.

Numerous empirical studies observed various choice anomalies (Camerer, 1995). This led Wrahmeman (1994) and others to argue that in some situations observed choices do not provide better infomation than introspection does. Several other papers have recently argued in fewor of utility concepts that are "more than choice-based", e.g. Gilboa \& Schmeicller (2001), Loomes \& Sugden (1982, 1987), Rabin (2000 footnote 3), Sen (1993 p. 498), and Timbergen (1991), and the interest in cardinal utility has increased.

In view of the importance of the cardinal versus ordinal debate, many other papers 
have been published following Alt (1936), both in psychology and in economics, giving sufficient conditions for a cardinal utility representation of preferences. They are reviewed in Section 2.4. The present chapter is of a similar nature and gives new conditions that are at the same time simpler, more general, and closer to empirical measurement procedures than those published before. All currently existing theorems follow as corollaries of this chapter.

This chapter is meant to be in the spinit of Alt (1936), not arguing for either ordinal or cardinal utility. The purpose is to show that even simpler conditions than traditionally thought need to be satisfied for obtaining cardinal utility, and, thus, to further clarify the differences between ordinal and cardinal utility. Nevertheless, those that consider preference differences as entirely meaningless, will not be convinced of the value of cardinal utility by the arguments of this chapter. But, if preference difference are per se not rejected, it is shown that relatively simple consistency conditions already imply cardinal utility.

The organization of the chapter is as follows. Section 2.2 states the main theorem, Section 2.3 provides some relaxations, Section 2.4 discusses related literature, and Section 2.5 concludes. The proofs can be found in the Appendix A of the chapter.

\subsection{Preference Foundations}

$X$ is a set of outcomes, e.g. monetary amounts, commodity bundles, or health states. The crucial assumption is that pairs of outcomes can be ordered by a difference relation $\succcurlyeq$ Pairs of outcomes are denoted by $a b$, where $a, b$ are elements of $X$. The following interpretation can be given to $a b \geqslant c d$ : The improvement obtained from receiving outcome $a$ instead of outcome $b$ is at least as good as the improvement obtained from receiving outcome $c$ instead of outcome $d$. An improvement can be positive or negative. Formally, $\succcurlyeq$ is a relation on $X \times X$. The symbols $\sim, \succ, \preccurlyeq$, and $\prec$ are defined us ustal. An underlying preference relation on $X$ is defined by $a \succcurlyeq^{\prime} b$ if $a b \Rightarrow b b$. Again, the symbols $\sim^{\prime}, \gamma^{\prime}, \xi^{\prime}$, and $\prec^{\prime}$ are defined as usual.

It would be desirable to have a function $u: X \rightarrow \mathbb{R}$ such that $u(a)-u(b) \geq u(c)-u(d)$ if and only if $a b \succcurlyeq c d$. Then, the $u$-differences represent the difference relation. For that reason, such a $u$ is called a diffenence representution. Furthermore, substitution shows that $u(a) \geq u(b)$ if and only if $a \succcurlyeq^{\prime} b$, that means the function $u$ also represents the preference relation $\vartheta^{\prime}$ and is, therefore, a utility function. 
To obtain a function as above, some assumptions must be satisfied. First, has to be a weok order on $X \times X$, that is, it has to be complete (i.e. for any two pairs ab, cd either $a b=a d$ or $a b \leqslant c d$ and transitive. Second, has to be wealy separable, that is, if $a c \geqslant b c$ then $a d ; b d$, and if $c a \succcurlyeq c b$ then $d a \succcurlyeq d b$, respectively, for all $a, b, c, d \in X$. In words, if who pairs in $X \times X$ only differ in one coordinate, then the difference between these two pairs solely depends on the noncommon outcome.

The next requirement uses standard sequences. A standard sequence is a finite or infinite sequence of elements $a^{0}, a^{1}, a^{2}, \ldots$ with $a^{1} x^{3} a^{0}$ and $a^{n+1} a^{n} \sim a^{n} a^{n-1}$ for all $n$. The improvement between any two subsequent elements in the sequence is always equally good. A standard secuence is increasing if $a^{0} \alpha^{\prime} a^{1}$ and decreasing if $a^{0}>^{3} a^{1}$. The difference relation $\succcurlyeq$ is Archimedean if any bounded standard sequence is finite (a standard sequence is bounded if there exist $b, c \in X$ with $b \succcurlyeq^{\prime} a^{n} \mho^{\prime} c$ for all $a^{n}$ in the standard sequence). This mearis thet there cannot exist an infinite increasing (decreasing) sequence of elements with fixed "stepsize", such that there is an ellement better (worse) than all elements in the swequence. If this were the case, the element better (worse) than all elements in the sequence would need to have infinite (minus infinite) utility.

For: the derivations, a "richness" assumption on $X$ is needed, called solvability. The difference relation is solvable if $x c \succ a b>z c$ implies the existence of a $y$ with $y c \sim a b$, and $c x>a b ; c z$ implies the existence of a $y$ with $c y \sim a b$, respectively, for all $a, b, c, x, z \in X$. Solwability holds in particular in the commonly assumed cases, where $z$ is continuous and

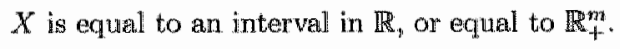

The following condition is straightforward. Neatrality holds if $a a \sim b b$ for all $a, b$. Loosely speaking, neutrality means that the improwement obtaimed from exchanging "the same for the same" is always equally good, i.e. there is no gam from trivial exchange.

'The abowe widely accepted and mostly' uncontroversial assumptions and conditions are not enough to guarantee the existence of a difference representation as desired. Nevertheless, and this is a crucial point of the chapter, the following additional condition is sufficient to derive a difference representation. The concatenation condition holds if $a b \sim a^{\prime} b$ ' and be $\sim b^{h} c^{t}$ imply ac $\sim a^{\prime} c^{\prime}$. The condition has an apperent interpretation. If the stepwise improvements from $a$ to $b$ and from $b$ to $c$ are exch equally good as the stepwise improvements from $a^{\prime}$ to $b^{\prime}$ and from $b^{\prime}$ to $c^{\prime}$, respectively, then the overall improvement from a to $c$ should be equally good as the overall improvement from $a^{\prime}$ to $c^{\prime}$. Semmelson (1938) already noted that the concatenation condition is a necessary condition for a difference represen- 
tation. In line with the ordinal view, Samuelson judged it as an arbitrary, i.e. undesired, restriction. The following theorem shows that the concatenation condition together with the other natural conditions implies the existence of a difference representation as desired. That is, the concatenation condition is the critical condition for cardinal utility.

Theorem 1 Let $\geqslant$ be a binary relation on $X \times X$ that satisfies solvabulity. Then the following two statements ane equivalent:

(1) There exists a difference representation $u: X \rightarrow \mathbb{R}$ with $u(a)-u(b) \geq u(c)-u(d)$ if and only if $a b \geqslant c d$.

(ii) $\succcurlyeq$ is an Archimedean, weakly separable, weak onder on $X \times X$ that satisfies neutrality and the concatenation condition.

The furction $u$ in (i) is cardinal, i.e. it is unique up to scale and location, and represents the underlying preference relation $\succcurlyeq^{\prime}$ an $X$.

The proof of Theorem $1 \mathrm{can}$ be found in the Appendix A of the chapter. The theorem demonstrates that Samuelson (1938) had the right intuition. The concatenation condition is precisely the point at issue.

\subsection{Generalizations}

This section starts with the following simple and useful observation: A two-dimensional additively separable representation satisfying neutrality is a difference representation. A relation $\succcurlyeq$ on $X \times X$ is additively separable if there exist functions $u, v: X \rightarrow \mathbb{R}$ such that $a b \geqslant c d$ if and only if $u(a)+v(b) \geq u(c)+v(d)$. Imposing neutrality in addition, leads to $v(\cdot)=-u(\cdot)+\gamma$ for some constant $\gamma$. Because $u(a)-u(b)+\gamma$ represents $\succcurlyeq$, $u(a)-u(b)$ also does, i.e. $u$ is a difference representation. Because of the wide range of results for additively separable representations, the above observation is useful. Any result on additively separable representations can be adapted to difference representations by simply adding neutrality. An example is giwen hereafter.

Assume that $=$ is an Archimedean, weakly separable, weak order on $X \times X$ that satisfies solvability. The hexagon condition is known as the weakest axiom sufficient to imply an additively separable representation (Wakker, 1989; Karni \& Safra, 1998; see 
also the Appendix B that discusses a problematic aspect of Karni \& Safra (1998)]. It holds if $a b \sim c d$ and $e d \sim c b \sim a f$ imply $c f \sim e b$. By adding neutrality, a difference representation is obtained. Therefore, Theorem 1 can be reformulated, by replacing the concatenation condition in (ii) by the hexagon condlition. Algebraic derivations show that, given separability and neutrality, the concatenation condition implies the hexagon condition, and, therefore, Theorem 1 is more general, if it is stated with the hexagon condition instead of the concatenation condition. The relationship between the two axioms is discussed further in Section 2.4. Although less general, the concatenation condition has the strong advantage that it has a more apparent interpretation in the context of difference representations than the hexagon condition.

In the rest of this section, it will be shown that there is an even more general condition than the hexagon condition, which would also be sufficient in Theorem 1.(ii). This more general condition has the advantage that it has an apparent interpretation, similarly as the concatenation condition, and that it is close to empirical measurement procedures. It has the disadvantage that it needs standard sequences and is, therefore, seemingly more complex than the concatenation condition.

In empirical measurement, it is common to let individuals indicate stepwise improvements all equally good as some benchmark interval $a^{1} a^{0}$ with $a^{1} \succ^{\prime} a^{0}$ (compare the introduction in Krantz, Luce, Suppes \& T versky (1971) and section 5 in Stigler (1950)). By this procedure an increasing standiard sequence $a^{0}, a^{1}, a^{2}, \ldots$ is obtained and $a^{n+1} a^{n} \sim a^{1} a^{0}$ for all $n$. The improvement of $a^{1}$ instead of $a^{0}$ serves, so-to-say, as a measuring rod. The improvement between any two subsequent elements in the sequence is always equally good as the improvement of receiving $a^{1}$ instead of $a^{0}$. To $a^{0}$ and $a^{1}$ some arbitrary values in the reals are assigned, say 0 to $a^{0}$ and 1 to $a^{1}$. Because the stepwise improvements are all equally good, value $i$ is then assigned to $a^{i}$. By this assignment of values, it is implicitly Essumed that any $k$ subsequent steps taken together are equally good as any other $k$ subsequent steps taken together, e.g. $a^{k} a^{0} \sim a^{k+1} a^{1} \sim a^{k+2} a^{2}$ etc. This assumption can be formalized as the standard-sequence condition, which is fulfilled if for all $k, m, n \in \mathbb{N}$ and all (increasing or decreasing) standard sequences $a^{0}, a^{1}, a^{2}, \ldots$ we have $a^{m+k} a^{n+k} \sim a^{m} a^{n}$. In words, if all one step improvements are equally good, then so are all k-step improvements.

The standard-sequence condition is not implied by meutrality and the concatenation condition. It is, however, in the presence of an uncontroversial condition, called the reversal condition, which is satisfied if $a b \sim c d$ implies $b a \sim d c$ for all $a, b, c, d \in X$. Given solvability, 
neutrality and the concatenation condition imply the reversal condition, which is proven in the Appendix A of the cliapter.

Except for the degenerate and trivial case of $a \sim^{\prime} b$ for all $a, b \in X$, neutrality is implied by the standard-sequence condition, but the reversal or the concatenation condition are not implied. Therefore, the standard-sequence condition is weaker and will lead to more general results than the other three conditions taken together.

The standard-sequence condition implies restricted wersions of the reversel and the concatenation condition, restricting the two conditions to standard sequences as follows. The midpoint condition is satisfied if $a b \sim b d$ implies ba $\sim d b$ for all $a, b, d \in X$ (the midpoint differs from the reversal condition only in that respect that $b=c$ ). The ssconcatenation condition is satisfied if $a^{i} a^{j} \sim a^{l} a^{\text {m }}$ and $a^{j} a^{k} \sim a^{m} a^{\text {m }}$ imply $a^{i} a^{k} \sim a^{l} a^{n}$ for all standard sequences $a^{0}, a^{1}, a^{2}, \ldots$ in $X$ and all $i, j, k, b, m, n \in \mathbb{N}$ (The abbreviation "ss" stands for standard sequence).

Lemma 2 For a weakly separable, weak onder $\Rightarrow$ on $X \times X$ such that $a, b \in X$ exist with $a \varkappa^{\prime} b$, the following two conditions are equiwalent:

(i) $\succcurlyeq$ satisfies neutrality, the midpoint condition, and the ss-concatenation condition.

(ii) $\succcurlyeq$ satisfies the standard-sequence condition.

In the proof of Theorem 1, where solvability is assumed, the concatenation condition is not needled in full strength, but only the midpoint and the ss-concatenation condition implied by the standard-sequence condition, which leads to the following observation.

Observation 3 Neutrality and the concatenation condition in Theorem 1.(ii) can be weakened to the standard sequence condition in the nontrivial case where $a, b \in X$ exist with $a x^{\prime} b$.

In this section two characterizations of difference representations alternative to the one in Theorem 1.(ii) via the concatenation condition have been provided, one using the hexagon condition and one using the standard-sequence condition as core axioms. Both of them are more general than the one in Theorem 1.(ii). It can be seen (see Appendix A of the chapter) that neutrality and the hexagon condition imply the standard-sequence condition but not the other way around, so that the characterization wia the standardsequence condition is, indeed, the most general result. 
Athough the standard-sequence condition gives the most general result, the author has chosen to pressent the main theorem (Theorem 1) via the concatenation condition. The tudvantage is that neutrality and the concatenation condition are simpler and mone accessible. On the other hand, the standard-sequence condition has the advantage that it is closer to empinical measurement procedures for elicitimg utility differences. In the next section, other charactenzations of difference representations that appeared in the literature are discussted. Besides stronger structural assumptions, the matjor difference in these approaches is that stronger axioms are used than the concatenation or the standard-sequence condition. All of these axioms imply the concatenation condition and the standard sequence condition. The characterizations in thils chapter are, therefore, common generalizations of all approaches in the literature, and simplify and unify the existing characterizations of cardimal utility.

\subsection{Related Literature}

In this section different axioms are discussed that appeared in the literature before. Figure 2.1 is meant to clarify the organization, and summarizes the implications (indicated by $\Rightarrow$ ) between the axioms defined hereafter. This will help the reader to keep the orientation in the rest of this section.

The implications in Fugure 2.1 do not invoke solvability and, therefore, the implications are elementary and apply to all weak orders. The proof can be found in the Appendix A of the chapter. For each " an example is provided.

Theorem 4.2 in Krantz, Luce, Suppes \& Tversky (1971) is closely related to Theorem 1, except that instead of neutrality and the concatenation condition these authors used stronger versions of the reversal and the concatenation condition, which imply, in particular, weak separability. The strong neversal condition holds if $a b \neq c d$ implies $b a \leqslant d c$. The strong concotenation condition holds if $a b \geqslant a^{\prime} b^{\prime}$ and $b c=b^{\prime} c^{\prime}$ imply $a c \neq a^{\prime} c$. In words, the strong reversal condition is a preference version of the reversal condition, and the strong concatenation condition is a preference version of the concatenation condition. By applying the strong reversal and the strong concatemation condition twice to the indifferences in the reversal and the concatenation condition, once replacing each indifference by " $"$, "and once by replacing each indifference by " $;$ " it follows that the strong reversal condition implies the reversal condition, and that the strong concatenation condition 
Let be a weak order on $X \times X$. Then,

Neutrality \& Thomsen $\Rightarrow$ crossover $\Rightarrow$

neutrality, reversal \& concatenation $\Rightarrow$

neutrality \& hexagon $\Rightarrow$ standard-sequence.

Even under neutrality and reversal,

Standard-sequence hexagon

concatenation crossover Thomsen.

Figure 2.1: Relations between the different conditions

implies the concatenation condition. Furthermore, the strong reversal condition also implies neutrality. Therefore, the standard-sequence condition is also implied. Because of these relations, the theorem in Krantz, Luce, Suppes \& Tversky (1971) can be obtained from Theorem 1 or Observation 3 as a corollary. For the converse, it is not too hard to see that weak separability plus neutrality, the reversal, and the concatenation condition do not imply the strong reversal condition. More interestingly, even given the strong reversal condition, those conditions do not imply the strong concatenation condition for general weak orders, as the following example shows. Theorem 1 and Observation 3 are, therefore, more general than Theorem 4.2 in Krantz, Luce, Suppes $\&$ Tversky.

Example 4 Let $X=\{a, b, c, d\}$. Define $\succcurlyeq$ on $X \times X$ by $a d \succ b d \succ a c \succ a b \succ b c \succ c d \succ$ $a a \sim b b \sim c c \sim d d \succ d c>c b>b a \succ c a ; d b \succ d a$. Weak separability, neutrality, the reversal condition, the concatenation condition, and even the strong reversal condition are fulfilled, but not the strong concatenation condition, because $a b \succ b c$ and $b c \succ c d$, but $a c \prec b d$.

Theorem 5.3 in Wakker (1988) also used the strong reversal and the strong concatenation condition, but in addition, instead of the Archimedean axiom and solvability, he assumed a continuity axiom and a connected domain $X$, which leads to a continuous utility 
function in the difference representation. These continuity assmmptions imply solvability and the Archimedean property (see the proof of Theorem 6.14 in Krantiz, Lnce, Suppes \& Twersky, 1971). The firsit paper that introduced a version of the strong concatenation condition, in combination whth stronger structural assumptions, was Alt (1936).

The most frequently usied, appealing axiom is the following. The crossover condition is fulfilled if $a b \sim$ cd implies $a c \sim b d$. For the crossover condition, there is also a preferences version. The strong crossover condition holds if $a b=c d$ implies $a c$ bd. The strong crossover condition appeared in Suppes \& Winet (1955), Davidson \& Marschak (1956), Scott \& Suppes (1958), Suppes \& Zinnes (1963), Debreu (1958), and Pfanzagl (1968). The indifference version was introduced by Shapley (1975). In the latter paper, Shapley proved a result similar to Theorem 1 , but with the crossover condition instead of neutrality, the reversal and the concatenation condition, and with continuity instead of solvability and the Archimedean property. Clearly, the strong crossover condition implies the crossover condition, but not the other way around. More interestingly, in the Appendix A of the chapter it will be shown that for general weak orders the crossover condition implies neutrality, the reversal condition, and the concatenation condition, and, therefore, also the standand-sequence condition. The reversed implication does not hold, i.e. the crossover condition is stronger and more restrictive than neutrality, the reversal condition, and the concatenation condition together.

A further related axiom can be found in Debreu (1960), of which a special case requires that $a b=a^{\prime} b^{\prime}$ and $c b \leqslant a^{\prime} c^{\prime}$ imply $a c^{\prime} \geqslant c b^{\prime}$. The weaker indifference version thereof is better known as the Thomen condition, which holds if $a b \sim a^{\prime} b^{\prime}$ and $a^{\prime} c^{\prime} \sim c b$ imply $a c^{\prime} \sim a b^{\prime \prime}$. The Thomsen condition and neutrality imply the crossover condition and, therefore, also the reversal, the concatenation, and the standard-sequence condition. The Thomsen condition usually plays a role in the derivation of additively separable representations, see Krantz, Luce, Suppes \& Tversky (1971) or Wakker (1989). As shown in Section 2.3, an additively separable representation satisfying neutrality is a difference representation.

Basu (1982) discussed conditions for the uniqueness result of cardinal utility given a conneted range, which is less general than solvability and the Archimedean axiom. His resultis follow as corollaries from those in the present chapter.

Although Figure 2.1 shows that even given neutrality and the reversal condition the five conditions, the Thomsen, crossover, concatenation, hexagon, and standard-sequence condition, are not equiwalent in general, the following remark shows that they are, nevertheless, 
equivalent in the case that the conditions are restricted to holld on standard sequences. For the remark, the following convention is introduced, where ... standls for reversal, Thomsen, crossover, concatenation, or hexagon, respectively. The $s s \%$... condition is satisfied on $X$ if the $\ldots$ condition is satisfied on every standard sequence $a^{0}, a^{1}, a^{2}, \ldots$ in $X$.

Remark 5 Let $\succcurlyeq$ be a weak onder on $X \times X$, such that $a$ w' b for some $a, b \in X$. The following five conditions are equivalent.

(i) $\succcurlyeq$ satisfies neutrality and the ssw Thomsen condition.

(ii) $\succcurlyeq$ satisfies the ss-crassover condition.

(wii) $\succcurlyeq$ satisfies neutrality and the ss-reversal and the ss-concatenation condition (the ssreversal condition can be shown to be equivalent to the midpoint condition).

(iv) $\succcurlyeq$ satisfies neutrality and the ss-hexagon condition.

(v) $\succcurlyeq$ satisfies the standard sequence condition.

Because of Observation 3, any of the conditions $(i)$ - (u) in Remark 5 carn replace neutrality, the reversal and the concatenation condition in Theorem 1.(ii) if $X$ is such that $a \nsim^{\prime} b$ for some $a, b \in X$.

A surprising feature of Theorem 1 and Observation 3 is that these are joint generalizations of all existing representation theorems for difference representations, and thereby unify all earlier derivations.

\subsection{Conclusion}

This chapter has organized and depicted the logical relations between all existing preference foundations for utility differences, and has provided unifying generalizations. The main theorem is a representation theorem for difference representations. It primarily builds on Theorem 4.2 in Krantz, Luce, Suppes \& Tversky (1971) and on Shaplley (1975). Observation 3 has given a further generalization of Theorem 1 . The central axioms, the concateration and the standard-sequence condition, are closely related to empirical measurement procedures. 
Whether utility is ordinal or cardinal depends on the acceptance of the characterizing axioms. As usual, some of these are uncontroversial and some are critical. The concatenation and the standard-sequence condition belong to the latter group. However, these conditions are as weak as possible, and may, thereby, further clarify the different nature of ordinal and cardinal utility. Moreover, the two conditions can be used for empirical tests of cardinality.

\subsection{Appendix A: Proofs}

Lemma 6 Let $\Rightarrow$ be a weak order on $X \times X$ satisfying neutrality and weak separability. Then $a \succcurlyeq^{\prime} b$ if and only if $a c \geqslant b c$, respectively, if and only if $c b \succcurlyeq$ ca for all $a, b, c \in X$. Furthermore, the latter implies that if $a^{0}, a^{1}, a^{2}, \ldots$ is a standard sequence in $X$ with $a^{1} \succ^{\prime} a^{0}\left(a^{1} \prec^{\prime} a^{0}\right)$ then $a^{i+1} \succ^{\prime} a^{i}\left(a^{i+1} \prec^{\prime} a^{i}\right)$ for all $i$.

Proof: $a \succcurlyeq b$ is equivalent to $a b \succcurlyeq b b$. Weak separability implies that the latter is equivalent to $a c \succcurlyeq b c$ for all $c \in X$. Furthermore, neutrality implies that $a \succcurlyeq b$ is equivalent to $a b \succcurlyeq a a$. Weak separability implies that the latter is equivalent to $c b \succcurlyeq c a$ for all $c \in X$.

Let $a^{a}, a^{1}, a^{2}, \ldots$ be a standard sequence in $X$ with $a^{l} \succ^{\prime} a^{0}\left(a^{1} \prec^{\prime \prime} a^{0}\right)$. Then $a^{i+1} a^{i} \sim$ $a^{1} a^{0} \succ(\prec) a^{0} a^{0} \sim a^{i} a^{i}$ for all $i$, i.e. $a^{i+1} \succ^{\prime} a^{i}\left(a^{i+1} \prec^{\prime} a^{i}\right)$.

Lemma 7 Let $\succcurlyeq$ be a weak orter on $X \times X$ that is weakly separable and solvable, and that satisfies neutrality and the concatenation condition. Then the reversal condition is also satisfied.

Proof: For a contradiction assume $a b \sim c d$, and $b a \succ d c$ for some $a, b, c, d \in X$. Three cases can occur.

Case 1: Assume $b a \succ d c \succcurlyeq b b$. Solvability implies that there exists an $x \in X$ with $b x \sim d c$. The concatenation condition applied to $a b \sim c d$ and $b x \sim d c$ implies $a x \sim c c$. Neutrality implies $a x \sim x x$. Lemma 6 implies $a \sim x$ and $b a \sim b x \sim d c$, contradicting $b a>d c$.

Case 2: Assume $b b \succ b a \succ d c$. Neutrality implies $d d \succ b a \succ d c$. Solvability implies that there exists an $x \in X$ with $b a \sim d x$. The concatenation condition applied to $a b \sim c d$ and $b a \sim d x$ implies $a a \sim c x$. Neutrality implies $c c \sim c x$. Lemma 6 implies $c \sim x$ and $b a \sim d x \sim d c$, contradicting $b a \succ d c$. 
Case 3: Assume $b a \succ b b \succ d c$. Neutrality implies $d d \succ d c$. Weak separability implies $a a>a b$ and $c d \succ c c$. Neutrality implies $c d \succ c c \sim a a \succ a b$, contradicting $a b \sim c d$.

All three cases lead to a contradiction. Therefore, $b a \preccurlyeq d c$. Similar contradictions can be inferred when $b a \prec d c$ is assumed, i.e. $b a \sim d c$.

Proof of Lemma 2: (ii) $\Rightarrow$ (i) : Assume $\geqslant$ satisfies the standard-sequence condition. First, neutrality is derived. Let $a, b \in X$. If $a \alpha^{\prime} b$, consider the special standard sequence $a^{0}, a^{1}$ with $a^{0}=a$ and $a^{1}=b$. The standard-sequence condition implies that $a^{0} a a^{0} \sim a^{1} a^{1}$, i.e. $a a \sim b b$. If $a \sim b$ then there exists a $c \in X$ with $a \sim^{\prime} c \alpha^{\prime} b$. As before, it can be inferred that $a c \sim c c \sim b b$. Neutrality is satisfied.

Second, the midpoint condition is derived. Let $a, b, c, d \in X$ with $a b \sim b c$. If $a x^{\prime} b$, consider the special standard sequence $a^{0}, a^{1}, a^{2}$ with $a^{0}=c, a^{1}=b$ and $a^{2}=a$. The standard-sequence condition implies that $a^{1} a^{2} \sim a^{0} a^{1}$, i.e. $b a \sim c b$. If $a \sim^{y} b$, then we have $b a \sim a a$ and $b c \sim a b \sim b b$. Weak separability implies $c c \sim c b$. Neutrality gives $b a \sim c b$. The midpoint condition is satisfied.

Third, the ss-concatenation condition is derived. Let $a^{0}, a^{1}, a^{2}, \ldots$ be a standard sequence and let $a^{i} a^{j} \sim a^{l} a^{m}$ and $a^{j} a^{k} \sim a^{m} a^{n}$. The standard-sequence condition implies that $a^{i} a^{j} \sim a^{l} a^{l-i+j}$. Lemma 6 implies that $a^{m} \sim^{l} a^{l-i+j}$. Furthermore, Lemma 6 implies that $a^{p} \sim a^{q}$ only if $p=q$, i.e. $i-j=l-m$. Similarly, $j-k=m-n$. We have $i-k=l-n$. The standard-sequence condition implies $a^{i} a^{k} \sim a^{l} a^{n}$. The ss-concatenation conclition is satisfied.

(i) $\Rightarrow$ (ii) : Let $a^{0}{ }_{n} a^{1}, a^{2}, \ldots$ be a standard sequence. An inductive reasoning shows that it suffices to prove $a^{m+1} a^{n+1-1} \sim a^{m} a^{n}$. We prove the latter by induction on $|m-n|$. For $|m-n|=0$ the hypothesis is true by neutrality. For $m-n=1$ the hypothesis is true by the definition of the standard sequence. For $m-n=-1$ we have to show that $a^{m+1} a^{m+2} \sim$ $a^{m} a^{m+1}$. The definition of the standand sequence implies that $a^{m+2} a^{m+1} \sim a^{m+1} a^{m}$. The midpoint condition implies $a^{m+1} a^{m+1} \sim a^{m} a^{m+1}$ and the case $m-n=-1$ is done. Assume it is already shown for some $l \geq 2$ that $a^{m+1} a^{n+l} \sim a^{m} a^{n}$ if $|m-n|<l$. Assume $m-n=l$. Then $a^{n+1} a^{n+2} \sim a^{m n} a^{n+1}$ and $a^{n+2} a^{n+1} \sim a^{n+1} a^{n}$. The ss-concatenation condition implies $a^{m+1} a^{n+1} \sim a^{m} a^{n}$. Assume $m-n=-l$. Then $a^{m+1} a^{n} \sim a^{m n} a^{n-1}$ and $a^{n} a^{n+1} \sim a^{n-1} a^{n}$. The ss-concatenation condition implies $a^{m+1} a^{n+1} \sim a^{m} a^{n}$. The induction is completes.

Remark: The restriction in Lemma 2 on $X$ that $a, b \in X$ exist with $a w^{\prime} b$ has the following reason. If we had $a \sim^{\prime} b$ for all $a, b \in X$, then the standard-sequence condition 
does not imply neutrality (E.g. $X=\{a, b\}$ and $a b \sim b b>b a \sim a a$ is possible). But, neutralty is the crucial condition which gives a fanction $w$ conetant on $X$, as desired in such a sitituation.

Proof of Theorem 1: The implication $(i) \Rightarrow(i)$ is straight forward. Left to show is the implication $(i i) \Rightarrow(i)$.

Within the rest of the proof, sometimes claims are made, of which the proofs are elaborated in brackets like 0 . These elaborations are usually of a technical nature using solvability and can be skipped.

Assume that $₹$ is an Archimedean, weakly separable, weak order on $X \times X$ that is solvable and satisfies neutrality and the concatenation condition. Lemma 7 implies that the reversal condition is satisfied. In the rest of the proof, we will only use the midpoint condition, which is implied by the reversal condition and the weak concatenation condition, which is implied by the concatenation condition.

CASE 1: Assume that $a \sim^{\prime} b$ for all $a, b \in X$. Neutrality implies $a b \sim b b \sim d d \sim c d$ for all $a, b, c, d \in X$. Let $u$ be any function that is constant on $X$. Then, $u$-differences represents the difference relation.

CASE 2: Assume that there exist $a, b \in X$ with $a x^{\prime} b$. A maximal standard sequence with respect to the mesh $a^{1} \succ^{\prime} a^{0}$ is defined to be a sequence $\ldots, a^{-2}, a^{-1}, a^{0}, a^{1}, a^{2}, \ldots$ with $a^{i+1} a^{i} \sim a^{1} a^{0}$ for all $i$ that has maximal length, i.e. if there exist an isuch that $a^{i}$ is defimed but not $a^{i+1}$, then there exists no $c \in X$ writh $c a^{i} \sim a^{\mathrm{l}} a^{0}$, and similarly, if there exist a $j$ such that $a^{j}$ is defined but not $a^{j-1}$, then there exists no $d \in X$ with $a^{j} d \sim a^{1} a^{0}$. Because of solvability, $a^{n+11}$ exists whenever $\left[a^{n}\right.$ exists and there is an element $b$ with $\left.b a^{n} \succcurlyeq a^{1} a^{0}\right]$, and $a^{-(n+1)}$ exists whenever $\left[a^{-n}\right.$ exists and there is an element $b$ with $a^{-n} b \succcurlyeq a^{2} a^{0}$ ]. An element $a$ is maximal, if $a \succcurlyeq b$ for all $b \in X$, and minimal, if $a \preccurlyeq b$ for all $b \in X$. In the rest of the proof, $r \in X$ is a nonmaximal fixed element.

REASONING 1 : Let $. ., a^{-2}, a^{-1}, a^{0}, a^{1}, a^{2}, \ldots$ be a maximal standand sequence. We show that $a^{\text {ratk }} a^{n+k} \sim a^{3 n} a^{n}$ for all integers $m, n, k$. Let $a=\min \{m, n, m+k, n+k\}$, $j=\max \{m, n, m+k, n+k\}$ and consider the standard sequence $b^{0}, b^{1}, b^{2}, \ldots, b^{j-i}$ with

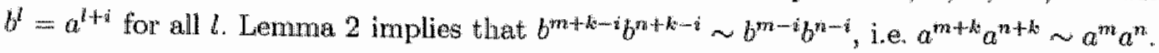

CASE 2.1: In this subcase a representing function is constructed for the case that an $a w^{\prime} r^{\prime}$ exists such that $b \succcurlyeq a$ for all $b y^{\prime} r$ (remember, $r \in X$ is fixed and nommaximal). 
This case is called the equally spaced case. Assume $a \succ^{\prime} r^{\prime}$ has the latter property. Let $\ldots, a^{-1}, a^{0}, a^{1}, \ldots$ be a maximal standard sequence with respect to the mesh $a \gamma^{\prime} r$, where $a^{1}=a$ and $a^{0}=r$.

Next, it is shown that for each $b$ an $i$ with $b \sim^{\prime} a^{i}$ exists. For a contradiction, assume that there is a $b$ with either $a^{i+1} \succ^{\prime} b \succ^{\prime} a^{i}$ for some $i$, or $b \succ^{\prime} a^{i}$ and $a^{i+1}$ does not exist $\left(a^{i}\right.$ is the maximal element in the standard sequence), or $a^{i+1} \chi^{\prime} b$ and $a^{i}$ does not exist ( $a^{i+1}$ is the minimal element in the standard sequence). Only the case $a^{i+1} \succ^{\prime} b \succ^{\prime} a^{i}$ for some $i$ is treated, the other two cases are similar. We have $a^{0} a^{0} \sim b b \prec a^{i+1} b \prec a^{i+1} a^{i} \sim a^{1} a^{0}$, where the second " $\prec$ " follows from Lemma 6. Solvability implies that there is a $c$ with $a^{i+1} b \sim c a^{0}$ and again Lemma 6 implies $a=a^{1} \succ^{\prime} c \succ^{\prime} a^{0}=r$, which contradicts the equally spaced case.

Define $u: X \rightarrow Z$ by $u(b)=i$ if $b \sim a^{i}$. Lemma 6 and Reasoning 1 imply that $a^{k} a^{j} \geqslant a^{k} a^{l}$ if and only if $i-j \geq k-l$, i.e. if and only if $u\left(a^{i}\right)-u\left(a^{j}\right) \geq u\left(a^{k}\right)-u\left(a^{l}\right)$. This implies that $u$-differences represent the difference relation $\succcurlyeq$ on $X \times X$.

CASE 2.2: Assume $X$ is such that for every $a \succ^{\prime \prime} r$ there exists a $b$ with $a \succ^{\prime \prime} b \succ^{\prime \prime} r$. This case is called the densely spaced case. In a first step, a function $u: X \rightarrow R$ is constructed that is shown to be a representing function in a second step. From now on, we may assume that $r$ is also nonminimal (the latter can be done because if no such $r$ would exist the equally spaced case applies).

STEP1: Let ..., $a^{-1}, a^{0}, a^{1}, \ldots$ be a maximal standard sequence with respect to the mesh $a^{1} \succ^{\prime} x^{\prime}=a^{0}$, where $a^{n}$ is arbitrary. For each $b \succcurlyeq^{\prime} r$ let $z_{b a}$ be the maximal integer with $b \succ^{\prime} a^{z_{b a}-1}$, and for $b \prec r$ let $z_{b a}$ be the minimal integer with $a^{z_{b a}} \succcurlyeq^{\prime} b$. The Archimedean axiom implies that $z_{b b a}$ exists.

REASONING 2: Let $\ldots, c^{-1}, c^{0}, c^{1}, \ldots$ be a second maximal standard sequence with respect to the mesh $c^{1} \succ^{\prime} r=c^{0}$, where $c^{1}$ is arbitrary. Next, it is shown for all integers $i, j$ that if $a^{i} \aleph^{\prime}\left(\alpha^{\prime}\right) c^{j}$, then for $n \geq 1$ we have $a^{i n} \alpha^{\prime \prime}\left(\prec^{\prime}\right) c^{j n}$ (if $a^{i n}$ and $c^{j n}$ exist), which is proven inductively. Assume that $a^{i} \preccurlyeq^{\prime}\left(\alpha^{\prime}\right) c^{j}$ and $a^{i k} \leqslant\left(\alpha^{\prime}\right) c^{j k}$. We show that $a^{i(k+1)} \leqslant^{\prime}\left(\prec^{\prime}\right) c^{j(k+1)}$. Lemma 6 and Reasoning $\mathbb{1}$ imply that $a^{i(n+1)} a^{i n} \sim a^{i} a^{0} \leqslant(<) c^{i} c^{0} \sim$ $c^{j(n+1)} e^{j n} \preccurlyeq(\prec) c^{j(n+1)} a^{i n}$. Lemma 6 implies $a^{i(n+1)} \leqslant\left(\alpha^{\prime}\right) c^{j(n+1)}$.

Furthermore, if $a^{i} \preccurlyeq^{\prime \prime}\left(\alpha^{\prime}\right) c^{j}$, then for $m \leq-1$ we have $a^{i m} \xi^{\prime}\left(\zeta^{\prime}\right) c^{j m}$ (if $a^{\text {tm }}$ and $c^{j m}$ exist), as is shown next. First, the latter is shown for the case $m=-1$. Lemma 6 \& Reasoning 1 imply that $a^{0} a^{-i} \sim a^{i} a^{0} \leqslant(<) c^{j} c^{0} \sim c^{0} c^{-j}$, i.e. because of Lemma 6 
$a^{-i} \succcurlyeq^{\prime}\left(\succ^{\prime}\right) c^{-j}$. Next, $m \leq-1$ is arbitrary. We already know that $a^{-i} \succcurlyeq^{\prime}\left(\succ^{\prime}\right) c^{-j}$. By the first pait of Reasoning 2 we know that $a^{-i|m|} \nvdash^{\prime}\left(\succ^{\prime}\right) c^{-j|m|}$, i.e. $a^{i m} \succcurlyeq^{\prime}\left(\zeta^{\prime}\right) c^{i m m}$.

We define an infinite sequence $a_{1} \succ^{\prime} a_{2} \succ^{\prime \prime} a_{3} \succ^{\prime \prime} \ldots \succ^{\prime} r$ recursively as follows (by $\ldots, a_{i}^{-1}, a_{i}^{0}, a_{i}^{1}, \ldots$ we denote the maximal standard sequence with respect to the mesh $a_{i} \succ^{\prime} r$, where $a_{i}^{ \pm}=a_{i}$ and $\left.a_{i}^{0}=r\right)$. The first element of the sequence $a_{1} \succ^{\prime} r$ is chosen arbitrarily, but such that $\alpha_{1}^{2}$ and $a_{1}^{-1}$ exist (Such an $a_{1}$ can be found because $r$ is neither minimal nor maximal, and by making use of solvability and the densely spaced property. In particular: Let $x \succ^{\prime} r$. Choose $y$ such that $x \succ^{\prime} y \succ^{\prime} r$. If $y^{2}$ exists then define $\bar{a}=y$. If $y^{2}$ does not exists then $y r \succ x y \succ r r$, and solvability implies the existence of a $y \succ^{\prime} z \succ^{\prime} r^{\prime}$ with $x y \sim z r$ and $x z \succ z r \succ r r$. Solvability implies the existence of $z^{2}$. Define $\bar{a}=z$. Finally, if $\bar{a}^{-1}$ exists then define $a_{1}=\bar{a}$. Otherwise choose a $w \prec^{\prime} r$. We know $\bar{a} r \succ r w \succ r$. Solvability implies that there exists an $\widehat{a}$ with $\bar{a} \succ^{\prime} \widehat{a} \succ^{\prime} r$ and $\widehat{a} r \sim r w$. Solvability implies that $\widehat{a}^{2}$ exists and $\widehat{a}^{-1}=w$. Define $a_{1}=\widehat{a}$. $)$.

If $a_{n i}$ is defined, $a_{n+1}$ is chosen such that $\left.a_{n+1}^{2}\right\}^{\prime} a_{n n}$. Similar as before, solvability and the densely spaced property imply that such an $a_{n+1}$ can always be found.

A function $u: X \rightarrow R$ will be constructed with the normalization $u\left(a_{1}^{0}\right)=0, u\left(a_{1}^{1}\right)=1$. Furthermore, $u\left(a_{1}^{i}\right)=i$ and $z_{b a_{1}}-1<u(b) \leq z_{b a_{1}}$ for all $b$. In particular, we will have $u(b)=\left\{\lim _{n \rightarrow \infty} \frac{z_{z_{a_{n}}}}{z_{a_{3} u_{32}}}\right.$.

Because $a_{n} \succcurlyeq^{\prime} a_{n+1}^{2}$ for all $n$, we have $a_{n} \succcurlyeq^{\prime} a_{n+1+i}^{2^{i}}$ and $a_{r i}^{-1} \preccurlyeq{ }^{\prime} a_{n+i}^{-2^{i}}$, as shown in Reasoning 2. From the Archimedean axiom it follows that for every $b \succ^{\prime \prime} r$ there is an $a_{i}$ with $b \succ^{\prime} a_{i}$ (if $b$ were " $\preccurlyeq \prime a_{i}$ " for all $i$ then because of Reasoning 2 also $b^{n}$ would be " $\preccurlyeq a_{i}^{n n}$ for all $i$ and all $n \in N$, i.e. $b^{n}$ would be " $\preccurlyeq$ ' $a_{i}$ " for all $i$, and wo would obtain an infinite bounded stanclard sequence, which is ruled out by the Archimedean axiom $\rangle$. Therefore, because of Reasoning $2, b \succ^{\prime} a_{i}^{z_{b a_{i}}-1} \succcurlyeq^{\prime} a_{i+n}^{\left(z_{b_{a}}-1\right) 2^{n}}$ for all n, i.e. $\lim _{n \rightarrow \infty} z_{b a_{n}}=\infty$. Similarly, for $b \prec^{\prime} r$

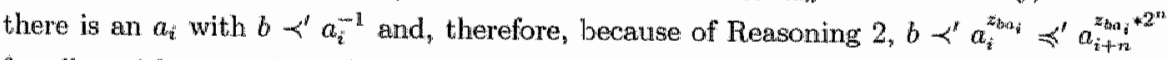
for all $n$ with $z_{b a_{i}}<0$, il.e. $\lim _{n \rightarrow \infty}, z_{b a_{n}}=-\infty$.

Furthermore, the Archimedlean axiom and solvability ensure that for all nonmaximal and nonminimal $b$ an $N_{b}$ exists such that for all $j \geq N_{b}$ the elements $a_{j}^{z_{b a_{j}}}$ and $a_{j}^{z_{b a_{j}}-1}$

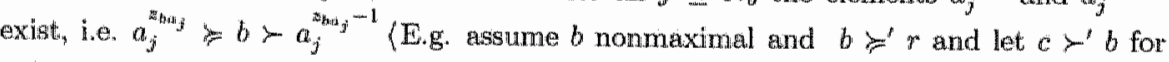
some c. If such an $N_{b}$ did not exist, there would be an infinite sequence $j_{1}<j_{2}<\ldots$ such that $a_{j_{k}, x_{k}}$ did not exist for all $k$, and, therefore, because of Lemma 6 and solvability

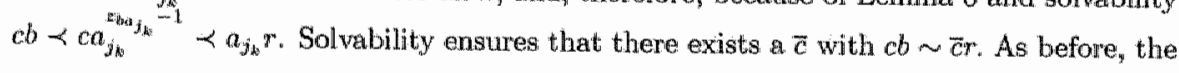


Archimedean axiom ensure that there exists an $a_{i}$ with $\vec{c} \succ^{\prime} a_{i}$, i.e. $c b>a_{i} r$. Therefore, because of Lemma $6, c b \succ a_{i+n} r$ for all $n \geq 1$, contradicting the existence of the above sequence $\left.j_{1}<j_{2}<\ldots.\right\rangle$.

REASONING 3: In this reasoning we will relate products of factors involving $z_{\text {bra }}$ and $z_{a_{j} a_{j+n}}$ to $z_{b a_{j+n}+}$. Let $b \succcurlyeq^{\prime} r$ be nonmaximal, and let $j \geq N_{b}$. In this case $z_{b a_{j+n}} \geq 0$ for

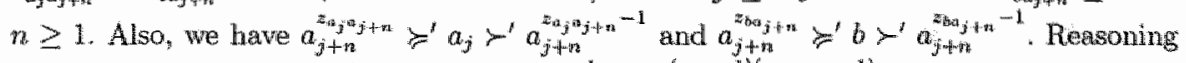

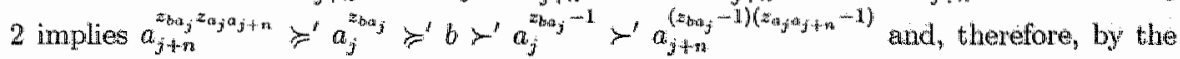
definition of $z_{b_{a_{j+n}+n}}, z_{b a_{j}} z_{a_{j} a_{j+n}}>z_{b a_{j+m}}-1 \geq\left(z_{b a_{j}}-1\right)\left(z_{a_{j} a_{j+n}}-1\right)$. By similar arguments it can be shown that also for maximal $\&$ we have $z_{b a_{j}} z_{a_{j} a_{j+n}}>z_{b a_{j ;}, n}-1 \succcurlyeq\left(z_{b a_{j}}-1\right)\left(z_{a_{j} a_{j+n}}-1\right)$.

Similarly, let $b \prec^{\prime} r$ be nonminimal, and let $j \geq N_{b}$. In this case $z_{b_{a_{j+n}+n}} \leq 0$ for $n \geq 1$. As

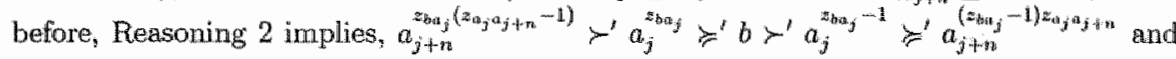
therefore $z_{b a_{j}}\left(z_{a a_{j} a_{j+n}}-1\right)>z_{b_{a_{j+n}+n}}-1 \geq\left(z_{b a_{j}}-1\right) z_{\mathrm{a}_{j} a_{j+n}+n}$. By means of similar arguments it can be shown that also for minimal $b$ we have $z_{b a_{j}}\left(z_{a_{j} a_{j+\cdots}}-1\right)>z_{k a_{j+m}}-1 \geq\left(z_{b a_{j}}-1\right) z_{a_{j} a_{j+\cdots}+\cdots}$.

For all $b \succcurlyeq r$, for $j \geq N_{b}$, and for all $n \geq 1$ we have, because of Reasoning 3 (once

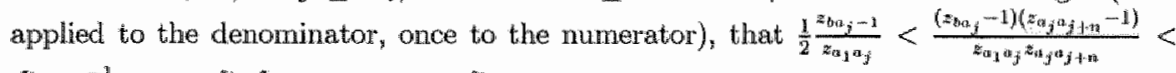

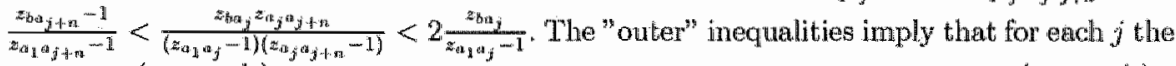

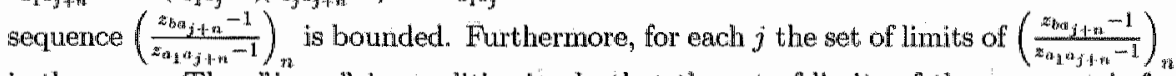
is the same. The "inner" inequalities imply that the set of limits of the sequence is for

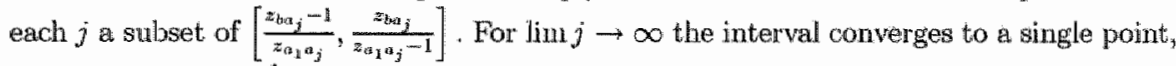

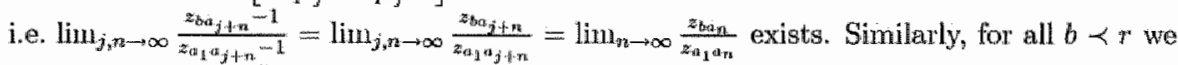

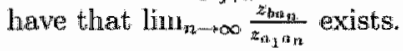

The function $a: X \rightarrow R$ is defined in the following way:

$$
u(b)=\left\{\lim _{n \rightarrow \infty} \frac{z_{b a_{n}}}{z_{a a_{1} a_{n}}}\right.
$$

STEP 2: Left to show is that differences of $u$, derived in Step 1, represent the difference relation. First, it is shown that $a b \sim c d$ implies $u(a)-u(b)=u(c)-u(d)$. Let $a b \sim c d$. For $a \sim b$ and $c \sim^{\prime} d$, clearly $u(a)-u(b)=u(c)-u(d)=0$. Assume next $a x^{\prime} b$ or $c w^{\prime} d$, say $c x^{\prime} d$. It can be seen that Reasoning 1 implies $\|\left(z_{a a_{i}}-z_{b a_{i}}\right)-\left(z_{c a_{i}}-z_{d a_{i}}\right) \mid \in\{0,1,2\}$ for all $i$ (E.g. assume $\left(z_{a a_{i}}-z_{b a_{i}}\right)-\left(z_{c a_{i}}-z_{d a_{i}}\right) \geq 3$. If $a_{i j}^{z_{d a i_{i}}-1}, a_{i}^{z_{b a_{i}}}, a_{i}^{z_{c a_{i}}}, a_{i}^{z_{d a_{i}}-1}$ exist, then Lemma 6 and Reasoning 1 imply $a b \succcurlyeq a_{i}^{x_{a a_{i}}-1} a_{\hat{i}}^{z_{b a_{i}}} \succ a_{i}^{z_{\Delta a_{i}}} a_{i}^{z_{d a_{i}}-1} \succcurlyeq c d$, yielding a contradiction. 


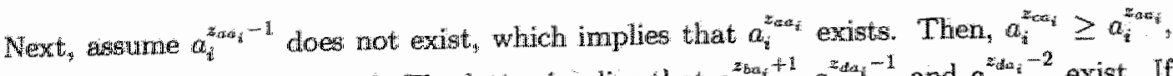

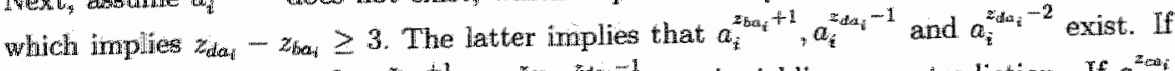

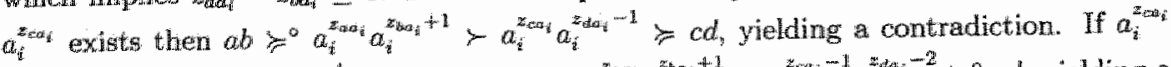

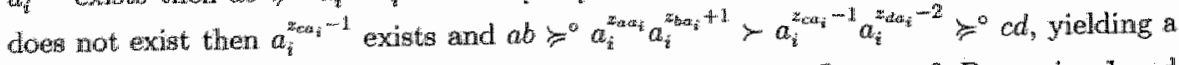
contradiction (the three " $"$ marked with o follow by using Lemma 6 , Reasoning 1 and solvabily). Other cases are similar. $\rangle$.

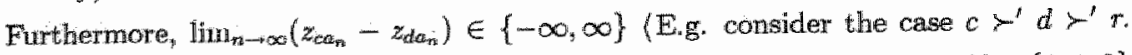

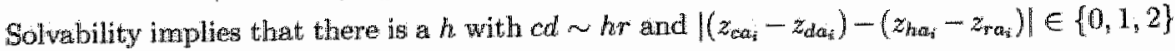

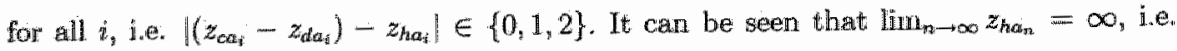
$\lim _{n \rightarrow \infty}\left(z_{n a n_{n}}-z_{d a_{n}}\right)=\infty$. Similar reasonings apply to other cases $\}$.

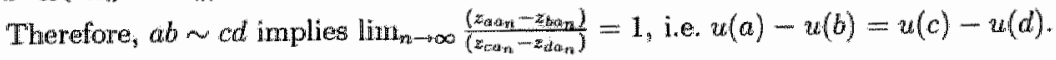

Second, it is shown that $a \succ^{\prime} b$ implies $u(a)>u(b)$. Let $a \succ^{\prime \prime} b$. Then, by similar: reasonings as before there exist $i, k$ with $a \approx^{*} a_{i}^{k} \gamma_{i}^{k+1} \alpha^{\prime} b$. Clearly, $u(a) \leq x\left(a_{i}^{k}\right)$ and $u\left(a_{i}^{\text {As }}\right) \leq u(b)$. We have $a_{i}^{k+1} a_{i}^{k} \sim a_{i}^{1} a_{i}^{0}$. By what was shown in the first part of

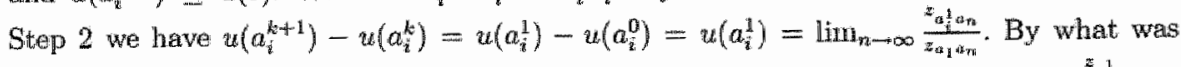

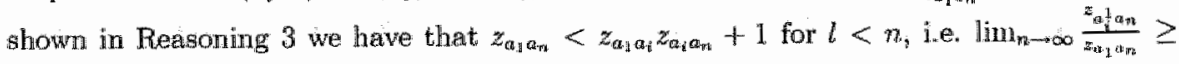

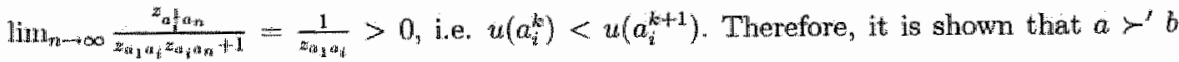
implies $u(a)>u(b)$.

The latter implication and solvability imply that if $a b \succ c d$, then $u(a)-u(b)>u(c)-$ $u(d)$. This suffices to show that $u$-differences represent the difference relation $\succcurlyeq$. The proof of $(i i) \Rightarrow(i)$ is complete.

Clearly, the function $u$ also represents the underlying preference relation. Uniqueness up to scale and location can be inferred from Theorem 4.2 in (Krantz, Luce, Suppes \& Twersky, 1971 ).

Proof of the Implications depicted in Figure 2.1: Neutrality \& Thomsen $\Rightarrow$ crossover: Let $a b \sim c d$. Neutrality implies $b b \sim c c$. Thomsen implies $a c m b d$.

Crossover $\Rightarrow$ nentrality, reversel \& concatenation: Clearly, $a b \sim a b$. Crossover implies $a a \sim b b$, i.e. neutrality is satisfied. Let $a b \sim c d$. Crossover implies $a c \sim b d$. Applying crossover to $b d \sim a c$ gives $b a \sim d c^{\text {, }}$ i.e. reversal is satisfied. Let $a b \sim a^{\prime} b^{\prime \prime}$ and $b c \sim b^{\prime} c^{\prime}$. Crossover implies that $a a^{\prime} \sim b b^{\prime}$ and $b b^{\prime} \sim c c^{\prime}$, je. $a a^{\prime} \sim a c^{\prime}$. Crossover implies $a c \sim a^{\prime} c^{\prime}$, i. e. concatenation holds.

Neutrality, reversal \& concatenation $\Rightarrow$ neutrality \& hexagon: Let ab $\sim c d$ and $e d \sim$ 
$c b \sim a f$. The first two indifferences, reversal, and concatenation imply $c a \sim e c$. The latter and the third indifference and concatenation imply of $\sim e b$. Therefore, hexagon is satisfied.

Neutrality \& hexagon $\Rightarrow$ standard-sequence: Let $\ldots, a^{-2}, a^{-1}, a^{0}, a^{1}, a^{2}, \ldots$ be a standard sequence. An inductive reasoning shows that it suffices to prove $a^{m+1} a^{n+1} \sim a^{m} a^{n}$. We prove the latter by induction on $|m-n|$. For $|m-n|=0$ the hypothesis is true by neutrality. For $m-n=1$ the hypothesis is true by the definition of the standard sequence. For $m-n=-1$ we have to show that $a^{m+1} a^{m+2} \sim a^{m m} a^{m+1}$. The definition of the standard sequence and neutrality imply that $a^{m+2} a^{m+1} \sim a^{m+1} a^{m}$ and $a^{m+1} a^{m} \sim$ $a^{m+1} a^{m+1} \sim a^{m+2} a^{m+2}$. Hexagon implies $a^{m+1} a^{m+2} \sim a^{m} a^{m+1}$ and the case $m-n=-1$ is completed. Assume it is already shown for some $l \geq 2$ that $a^{m+1} a^{n+1} \sim a^{m} a^{m}$ if $|m-n|<l$. Assume $m-n=l$. Then $a^{m-1} a^{n+1} \sim a^{n n} a^{n+2}$ and $a^{m+1} a^{n+2} \sim a^{m} a^{n+1} \sim a^{m-1} a^{n}$. Hexagon implies $a^{m+1} a^{n+1} \sim a^{m} a^{n}$. Assume $m-n=-l$. Then $a^{m+2} a^{n} \sim a^{m+1} a^{n-1}$ and $a^{m} a^{n-1} \sim a^{m+1} a^{n} \sim a^{m+2} a^{n+1}$. Hexagon implies $a^{m+1} a^{n+1} \sim a^{m} a^{n}$. The induction is complete.

For the rest of the proof, we assume that neutrality and the reversal condition are satisfied.

Standard-sequence $\Rightarrow$ hexagon: Let $X=\{a, b, c, d, e\}$ and $a e>a d \sim b e \succ a c \sim b d \sim$ $c e \succ a b \sim b c \succ c d \sim d e \succ a a \sim b b \sim c c \sim d d \sim e e \succ e d \sim d c \succ c b \sim b a \succ e c \sim d b \sim c a \succ$ eb $\sim d a \succ e a$. Neutrality, reversal and standard-sequence are fulfilled, but not hexagon, because $a d \sim b e$ and $c e \sim b d \sim a c$, but $b c \succ c d$.

Hexagon $\Rightarrow$ concatenation: Let $X=\{a, b, c, d\}$ and $d a \succ d b \succ c a \succ d c \sim c b \sim b a \succ$ $a a \sim b b \sim c c \sim d d \succ a b \sim b c \sim c d \succ a c \succ b d \succ a d$. Neutrality, reversal and hexagon are fulfilled, but not concatenation, because $d c \sim c b$ and $c b \sim b a$, but $d b \succ c a$.

Concatenation $\Rightarrow$ crossover: Let $X=\{a, b, c, d\}$ and $a d \succ b d \succ a c \succ a b ; c d \succ$ $b c \succ a a$. Furthermore assume reversal, concatenation and weak separability. The given preferences together with weak ordering and weak separability of $₹$ and with neutrality, reversal and concatenation already determine all preferences between any two pairs in $X \times X$. Becruse $a b \succ c d$ and $b d \succ a c$, crossover is not fulfilled.

Crossover Thomsen: Let $X=\{a, b, c, d, e\}$ and $a e \succ a d \succ b e \succ a c \sim c e \succ a b \succ$ de $\succ b d \succ b c \sim c d \succ a a \sim b b \sim c c \sim d d \sim e e \succ d c \sim c b \succ d b \succ e d \succ b a \succ e c \sim c a \succ$ $e b \succ d a \succ$ ea. Neutrality, reversal and crossover are fulfilled, but not Thomsen, because $a c \sim c e$ and $c b \sim c d$, but ad $\succ$ be. 
Proof of Remark 5: Because of the implications depicted in Figure 2.1 we already know that $(i) \Rightarrow(i i) \Rightarrow(i i i) \Rightarrow(i v) \Rightarrow$ (v) . Left to show is that $(v) \Rightarrow$ (i). Let $\Rightarrow$ satisfy the standard-sequence condition. Let $a^{0}, a^{1}, a^{2}, \ldots$ be a standard sequence. The standard-sequence condition implies that $a^{m+k} a^{m} \sim a^{n+k} a^{n}$ for all $k, m, n$. Furthermore, the standard-sequence condition implies neutrality (Lemma 2) and Lemma 6 implies that if $a^{m+k_{a}} a^{m} \sim a^{n+l} a^{n}$, then $k=l$. Assume that $a^{i} a^{j} \sim a^{k} a^{l}$ and $a^{m} a^{j} \sim a^{k} a^{n}$. Then $i-j=k-l$ and $m-j=k-n$, i.e. $i-n=m-l$. Therefore, $a^{i} a^{r} \sim a^{m} a^{l}$ and the Thomsen condition is satisfied within the standard sequence, i.e. $(v) \Rightarrow(i)$ is shown.

\subsection{Appendix B: Note on "Karni \& Safra (1998)"}

Karmi \& Safra (1998) proved that, under the algebraic approach to conjoint measurement, a binary relation $\succcurlyeq$ has an additive representation if and only if $\succcurlyeq$ satisfies the hexagon condlition, given some natural assumptions. Their result is a joint generalization of the corresponding result for the topological approach (Wakker, 1989), which imposes more restrictive structural assumptions, and of related results for the algebraic approach, which uses preference conditions that are more restrictive than the hexagon condition. Additive representations play an important role in the economic and the psychological literature. Karni and Safra provided the most general result for additive representations on two dimensions. From this all earlier results follow as corollaries. Therefore, a clarification of a problematic step in the proof is warranted.2

In the beginning of page 398 , the authors assume that $\widehat{a}$ and $\hat{b}$ can be chosen such that $(\widehat{a}, \widehat{b}) \sim\left(a^{\prime \prime}, b\right)$ and $G\left(\left(a, b^{\prime \prime}\right), \widehat{a}, \widehat{b}\right)$ is a grid. A grid requires that $(a, \widehat{b}) \sim\left(\widehat{a}, b^{\prime \prime}\right)$. To see that this is problematic, note that restricted solvability implies that there exists an $a^{*} \succ a$ such that $\left(a^{*}, b^{\prime \prime}\right) \sim\left(a^{\prime \prime}, b\right)(\sim(\widehat{a}, \widehat{b}))$. Because $(a, \widehat{b}) \sim\left(\widehat{a}_{3}, b^{\prime \prime}\right)$ and $(\widehat{a}, \widehat{b}) \sim\left(a^{*}, b^{\prime \prime}\right)$, $\widehat{a}$ would be the "midpoint" between $a$ and $a^{*}$ in terms of the function $\kappa_{i}(x)$, if an additive representation $\kappa(\cdot)+\sigma(\cdot)$ exists. Such midpoints exist under the topological approach, but not necessarily under the weaker algebraic approach. It is typical of the novelty of the algebraic approach that midpoints need not exist (Example III.6.8 in Wakker, 1989), which motivated this note.

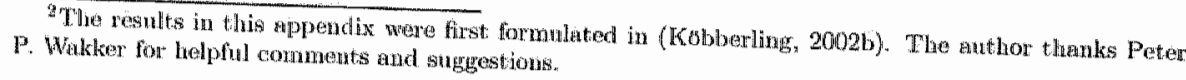


The described problem can be solved in the following way. ${ }^{3}$ It suffices to show that there exist $\widehat{a} \succ a$ and $\widehat{b} \succ b^{n}$ with $(a, \widehat{b}) \sim\left(\widehat{a}, b^{z}\right)$ and $(\widehat{a}, \widehat{b}) \preccurlyeq\left(a^{\prime \prime}, b\right)$. With this small adaptation and the "new" $\widehat{a}$ and $\widehat{b}$, the rest of the original proof goes through (note the following typos: line $9 \& 10$, replace each $\vec{a}^{\prime \prime}$ by $\vec{a}^{\prime}$; line 11 , replace $b$ by $\vec{b}$ ). In Figure 2 the point $(\hat{a}, \widehat{b})$ should lie below the indifference curve through $\left(a^{\prime \prime}, b\right)$.

Left to show is that $\widehat{a}$ and $\hat{b}$ as desired exist. Take $a^{*}$ as defined above. By the assumption, there exists an $\widetilde{a}$ with $a<\widetilde{a}<a^{*}$. By restricted solvability, there exists a $\widetilde{b} \succ b^{\prime \prime}$ with $\left(\tilde{a}_{,} \tilde{b}\right) \sim\left(a^{\prime \prime}, b\right)$. By restricted solvability, there is a $\tilde{b}^{\prime}$ with $b^{\prime} \succ \tilde{b}^{\prime} \succ b^{\prime \prime}$ and $\left(\widetilde{a}, b^{\prime \prime}\right) \sim(a, \widetilde{b})$. If $\widetilde{b^{\prime}} \preccurlyeq \widetilde{b}$, let $\widehat{a}=\widetilde{a}$ and $\widehat{b}=\widetilde{b}^{\prime}$. Otherwise, there is, by restricted solvability, an $\widetilde{a}^{t}$ with $\widetilde{a} \succ \widetilde{a}^{\prime} \succ a$ and $(a, \tilde{b}) \sim\left(\widetilde{a}^{\prime}, b^{\prime \prime}\right)$. In this case, let $\widehat{a}=\widehat{a}^{\prime}$ and $\widehat{b}=\widetilde{b}$.

\footnotetext{
${ }^{3}$ The choser way of correcting the proof was suggested by Safna and Kami in porsonal communicotion.
} 


\section{Chapter 3}

\section{A Tool for Qualitatively Testing, Quantitatively Measuring, and Normatively Justifying Expected Utility}

This chapter introduces a new preference condition that can be used to justify (or criticize) expected utility. The approach of this chapter is alteriative to Savage's, and is accessible to readers without a mathematical background. It is based on a method for deriving "comparisons of tradeoffs" from ordinal preferences. Our condition simplifies previously-published tradeoff conditions, and at the same time provides more general and more powerful tools to specialists. The condition is more closely related to empirical methods for measuring utility than its predecessors. It provides a unifying tool for quantitatively measuring, qualitatively testing, and normatively justifying expected utility. ${ }^{1}$

\subsection{Introduction}

A central topic of debate in decision theory is the normative status of expected utility. Expected utility provides a general framework for decisions under uncertainty and was considered normative by its early contributors (Ramsey, 1931; de Finetti, 1937; won Neumann

\footnotetext{
'The results in this chaptar were first formulated in (Koblbering \& Waklker, 2002 a). Jondthan Baron and Han Bleichrodt made helpfil comments.
} 
\&. Morgenstern, 1944; Savage, 1954; Raiffa, 1968). Many have challenged the normative status of expected utility (Allais, 1953; Ellsberg; 1961; Epstein, 1992; Machina, 1982 ; Loomes $\mathrm{K}_{\mathrm{K}}$ Sugden, 1982; Schmeidler, 1989). The debate has usually centered around the plausiblity of the characterizing preference conditions.

Von Neumann \& Morgenstern (1944) provided a simple and appealing characterization of expected utillty under risk by means of their independence preference condition. Risk. refers to the special case of known probabilities. Economically more relevant is the case of uncertainty, where probabijities are unkmown. Characterizations and measurements are more complex for this cuse Savage (1954) provided a first characterization of expected utility for uncertainty. We propose an altermative characterization, based on a method for measuring utility under uncertainty. This method uses comparisons of "tradeoffs" (Wakker, 1984), by means of which orderings of utility differences can be inferred from ordinal preferences between acts. Wakker (1989) showed that expected utility holds if and only if such comparisons of tradeoffs do not lead to contradictions. A variation of this method, renamed tradeoff consistency, was used by Twersky \& Kahneman (1992) to characterize cumulative prospect theory. We simplify and generalize the tradeoff conditions published so far.

This chapter concentrates on the intuition of the tradeoff condition, leading to a new intuitive foundation for expected utility. In short, Savage's (1954) event-independent ordering of outcomes (P3) is extended to an event-independent ordering of strengths of preferences between outcomes, Mathematical generalizations will be given in the follow-up paper Kobberling \& Wakker (2002b, see Chapter 4).

While our approach is simpler than earlier approaches and, therefore, more easily accessible to monspecialists (compane LeRoy \& Wemer, 2000, Section 8.6), it is at the same time more general than earlier approaches in a mathernatical sense. It derives the same implications from weaker assumptions, thereby providing more powerful techniques" for specialigts. The obtained simplification allows for a new characterization, alternative to tradeof: consistency: Expected utility holds if and only if the rewealed "equivalences of tradeolfs ${ }^{\mathrm{k}}$ satisfy transitivity.

A further advantage of our tradeoff consistency is that it is closer to empirical measurements of utility than its predecessors, e.g. those in Wakker (1984, 1989, 1994), Tversky \& Kahnernan (1992), and Abdellaoui (2002). This chepter can, indeed, be considered the theoretical counterpart to the empirical utility measurement of Wakker \& Deneffe (1996). 
We hope that the tradeoff technique can appeal to a basic concept of cardinal utility, prior to risk or uncertainty, and can, thus, fomalize an intuition described by Broome (199: p. 147). A simular concept of utility was advocated by Harsanyi (1978) for expected utility, and by Allais (1953) and Loomes \& Sugden (1982) in their derivations of nonexpected utility models. The tradeoff technique may provide a link between risky ${ }^{2}$ utility, which it formally reveals, and other concepts of cardinal utillity (discussed by Fishburn, 1989; Ng, 1997; Samuelson, 1937 p. 161; Zeuthen, 1937), while renaining within the revealed-preference paradigm. In a follow-up paper, Abdellaow, Barrios, We Waker (2002) tested this claim experimentally. They found that risky utilities, measured by means of the techniques of this chapter, agree well whith riskless utilities measured through direct strength of preference questions, indeed. The latter are not based on revealed preferences but on direct judgments.

Like earlier derivations based on tradeoff consistency, our derivation can deal with finite state spaces, extending Savage (1954) who required an infinite state space. Our derivation can also deal with infinite state spaces, using Wakker's (1993b, Corollary 2.14) technique, generalizing Savage (1954) in allowing for atoms. Other extensions of Savage's expected utility to finite state spaces can be found in Grodal (1978), Nakamura (1990), Gul (1992), and Chew \& Karni (1994).

The tracleoff method can be used to empinically classify deviations from expected utility. For example, if tradeoff consistency can be violated in general but is never violated when the considered acts are "comonotonic" (defined in Schmeidler, 1989), then trankdependent model can be shown to apply (Köbberling \& Wakker, 2002b, see Chapter 4), If tradeoff consistency can be violated when the considered acts are comonotonic but only if they are not co-signed (co-signed means that there is no state yielding a gain under one act and a loss under another), then curmulative prospect theory holds (Kobberling \& Wakker, 2002b, see Chapter 4). Under betweenness theories (Dekel 1986, Epstein 1992), tradeof consistency is never violated within indifference classes (Gilboa, Schmeidler, \& Wakker, 2002, Lenma A.5), but can be between.

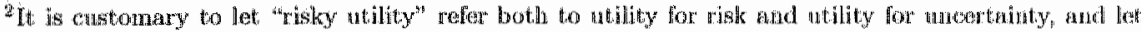

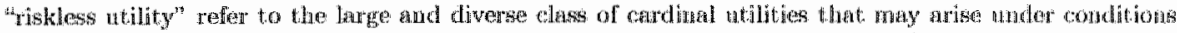
whithout risk, such as intertemporal or interpersonal aggregations. Although the teminology may be misleading tor the purposes of this chapter, which concerns general uncertanity it is so customary that we will adture to it:
} 


\subsection{The Intuition Underlying our Condition}

This section presents the derivation of expected utility that is alternative to Savage (1954), and is accessible to readers without a mathematical bachground. As a preparation, we discuss the measurements of strengths of preferences. These measurenents have been controversial in the economic literature. For example, assume that an agent judges that kifs strength of preference for $\$ 100$ instead of $\$ 0$ exceeds that for $\$ 1101$ instead of $\$ 1000$. Such subjective judgments cannot be revealed from observed choice. Whereas they may be psychologically meaningful (Pareto, 1906, p. 191 in 1971 translation), it became generally accepted that they should not be part of the economic domain (Hicks \& Allen, 1934).

This section proposes a different concept of strength of preference that can be revealed from preferences under uncertainty. We emphasize that these strengths of preferences are derived from ordinal empirical inputs only, and are not assumed as empirical primitives. Our approach, therefore, remains within the revealed preference paradigm. We will see that the mentioned revelations capture the intuition of expected utility and lead to a new foundation thereof. For simplicity of the presentation, only monetary outcomes are considered henceforth.

Assume that a clecision maker has to choose between state-contingent assets called acts. For instance, an act may be $(A, 310 ; B, 0)$, yielding $\$ 310$ if event $A$ obtains (e.g., candidate 1 will win the elections) and $\$ 0$ if event $B$ obtains (another candidate will win). Subjective expected utility requires that a probability $P$ is assigned to each event and a utility $U$ to each outicome. If no probabilities of events are known, subjective probabilities have to be assigned that best reflect the degree of belief of the decision maker. Actsine evaluated by their probability-weighted average utility, e.g. this is $P(A) U(310)+P(B) U(0)$ for the act just described. From available acts, the one with the highest expected utility is chosen.

In the following analysis, we do not assume expected utility but instead consider preference conditions that are argued to be reasonable for normative purposes. We then show that they imply expected utility. The intuitions underlying our analysis are similar to those in Loomes \& Sugden (1982), Broome (1991, p. 146/147), and Baxon (1994, Section 17.1.5). To explain the basic tool of this chepter, we consider the following two indiffer-

\footnotetext{
"We usually drop "subjective" henceforth.
} 


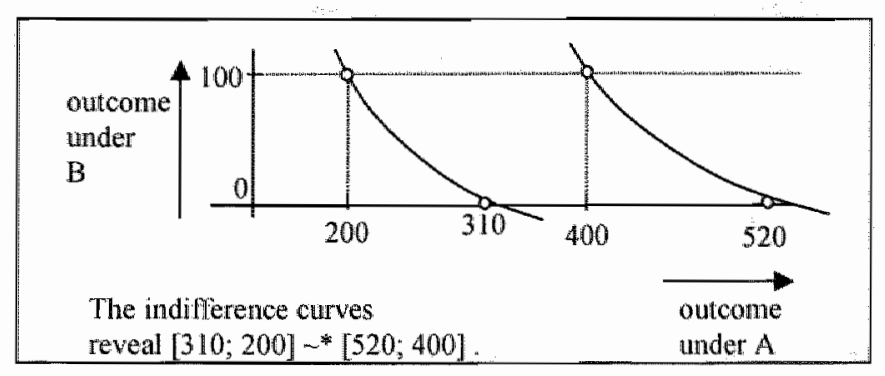

Figure 3.1: Indifference Curves

ences, depicted in Figure 3.1.

$$
\begin{aligned}
& (A, 310 ; B, 0) \sim(A, 200 ; B, 100) \text { and } \\
& (A, 520 ; B, 0) \sim(A, 400 ; B, 100) .
\end{aligned}
$$

In both indifferences, event $B$ provides an argument against the left act because then outcome 0 instead of 100 is received. Henceforth, $[0 ; 100]$ is a linguistic abbreviation of "receiving outcome 0 instead of outcome 100." In both indifferences, event $A$ provides an argument in favor of the left act corresponding to [310;200] (receiving outcome 310 instead of outcome 200) in the first indifference and to $[520 ; 400]$ in the second.

The strength of the argument against the left act provided by $[0 ; 100]$ depends, obviously, not only on how much worse it is to receive 0 instead of 100 but also on event $B$, in particular its perceived likelihood. The likelihood of $A$ is similarly relevant to the weight of the arguments provided by $[310 ; 200]$ and $[520 ; 400]$, respectively. At any rate, the first indifference suggests the following interpretation:

$([310 ; 200]$ contingent on event $A)$ exactly offsets $([0 ; 100]$ contingent on event $B)$.

Similar "statewise" comparisons are described by Tversky (1969 p. 41) and are central in regret theory (Loomes \& Sugden, 1982) and in Fishburn's skew-symmetric bilinear theory 
(Fishburn \& Lavalle, 1988). The second indiference can be interpreted likewise, in.

$([520,400]$ contingent on event $A)$ exactly offsets $([0 ; 100]$ contingent on event $B)$.

These two interpretations together suggest that $[520 ; 400]$ can do exactly the same as $[310 ; 200]$, ie offset $[0 ; 100]$ contingent on event $B$ whille being contingent on the same event $A$. We interpret this as $[310 ; 200]$ being an equally good improvement as $[520 ; 400]$, and use the notation

$$
[310 ; 200] \sim[520 ; 400]
$$

as a linguistic abloreviation of this interpretation. Note how $[0 ; 100]$ contingent on $B$ hass served ass a gauge to establish Eq. 3.5.

Asuming expected utility for now, the first indifference (Eq. 3.1) implies

$$
P(A)(U(310)-U(200))=P(B)(U(100)-U(0))
$$

(compare the interpretation in Eq. 3.3). The second indifference (Eq. 3.2) implies

$$
P(A)(U(520)-U(400))=P(B)(U(100)-U(0))
$$

(compare the interpretation in Eq. 3.4). We obtain the following quantitative counterpart to the indifference $\sim *$ in Eq. 3.5 .

$$
U(310)-U(200)=U(520)-U(400)
$$

We now return to the general analysis, where expected utility is not assumed, and formalize the concepts discussed so far. The relations $\sim$ " can be derived fron ohrer

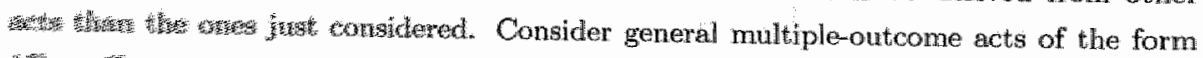

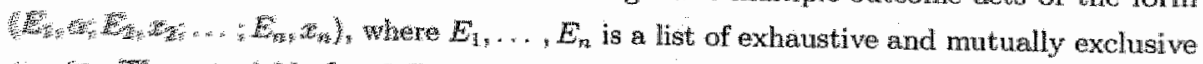

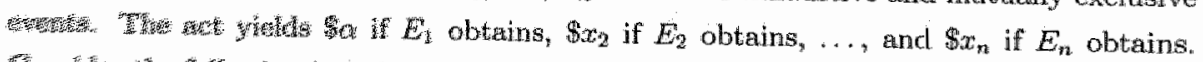

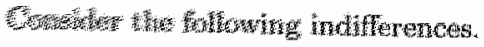

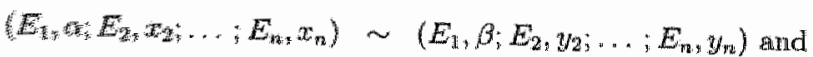

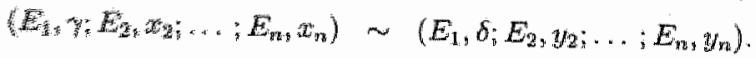


We formally write

$$
[\alpha ; \beta] \sim[\gamma ; \delta]
$$

whenever we can find events $E_{1}, \ldots, E_{n}$ and outcomes $x_{2}, \ldots, x_{n}, y_{2}, \ldots, y_{n}$ such that the above indifferences hold. The interpretation is the same as discussed at the beginning of this section. The two indifferences in Eqs. 3.1 and 3.2 are a special case of those in Eqs. 3.6 and 3.7 (take $n=2, A=E_{1}, B=E_{2}, \alpha=310, \beta=200, \gamma=520, \delta=400, x_{2}=$ $\left.0, y_{2}=100\right)$. In Eqs. 3.6 and $3.7,[\alpha ; \beta]$ and $[\gamma: \delta]$, contingent on $E_{1}$, offset the same gauge (receiving the $x$ outcomes instead of the $y$ outcomes) contingent on not- $E_{1}$. The latter gauge is more complex than what we saw before, with various outcomes and events involved, but this does not change the reasoning. By algebraic manipulations as before,

$$
U(\alpha)-U(\beta)=U(\gamma)-U(\delta)
$$

follows under expected utility.

If the interpretation of the relation $\sim^{*}$ is accepted, then transitivity of $\sim^{*}$ seems to be a reasonable requirement for normative purposes. That is, if $\{\alpha ; \beta]$ can offset the same gauge as $[\gamma ; \delta]$ in one situation, and $[\gamma ; \delta]$ can offset another "same gauge" as $\left[\sigma_{;} \tau\right]$ in another situation, then it seems reasonable that $[\alpha ; \beta]$ can offset the same gauges as $[\sigma ; \tau]$ in other situations.

In the same vein as the above reasoming, the relations $\sim$ * should not reveal contradictory orderings in the following sense. Assume that we have the two indifferences in Eqs. 3.6 and 3.7 , with $\alpha=315, \beta=200, \gamma=520$, and $\delta=400$ so that $[315 ; 200] \sim \sim^{*}[520 ; 400]$ follows. Assume that we also have the two indifferences at the beginning of this section, implying $[310 ; 200] \sim[520 ; 400]$. In a way similar to transitivity, this generates the undlesirable suggestion that [315;200] would be an exually good improvement as [310; 200], both being equally good as $[520 ; 400]$. To exclude such undesirable suggestions, we define tradeoff consistency as the requirement that not $\left.\left[\alpha^{*} ; \beta\right] \sim^{* \prime} \mid \gamma ; \delta\right]$ and $\left[\alpha_{n}^{*}, \beta\right] \sim^{*}[\gamma ; \delta]$ for an $\alpha^{\prime} \neq \alpha$ ( $\alpha^{\prime} \nsim \alpha$ for general nonmonetary outcomes; this chapter assumes monetary outcomes and monotonicity, implying that equivalence is the same as identity). In words:

Tradeoff consistency requires that, if one of the four outcomies in a $\sim^{*}$ relation is replaced by a nonequivalent outcome, then the $\sim$ " relation should not hold any more. 
As it turns out, transitivity and tradeoff consistency of $\sim$ * need not hold for general preferences. We will see that each of these two conditions in isolation holds, under some natural conditions, if and only if expected utility holds. In particular, the two conditions are equivalent. The mentioned natural conditions are weak ordering (completeness of preference for every pair of acts and transitivity), monotonicity (the more money the better), and continuity (small changes in outcomes do not generate large changes in preference).

Observation 8 Assume that preferences satisfy weak ordering, monotonicity, and continuity. The following three statements are equivalent.

(i) Subjective expected utility holds.

(ii) Tradeoff consistency holds.

(iii) $\sim *$ is transitivive.

If tradeoff consistency or transitivity of the revealed orderings of tradeoffs is deemed (un)desirable in an empirical or normative sense, then so should expected utility be. We sketch the proof of the theorem in the main text because it illustrates how to measure probabilities and utilities empirically. Topological and other technical aspects of the proof are elaborated in Köbberling \& Wakker (2002b, see Chapter 4), where the case of nonmonetary outcomes is also considered.

To define utility $U$, we set $U^{\gamma}\left(x^{0}\right)=0$ for some $x^{0}$ and $U\left(x^{1}\right)=1$ for some $x^{1}>x^{0}$. For all $j$ (also negative), we take $x^{j}$ s such that $x^{j} x^{j-1} \sim^{*} x^{1} x^{0}$, and define $U\left(x^{j}\right)=j$. If utility is bounded there will be bounds on the indexes $j$ for which such $x^{j}$ can be defined. Next, we refine the domain of $U$. For all $j$, we find $y^{j} y^{j-1} \sim^{*} y^{1} x^{0}$ in such a wey that $y^{2}$ coincides with $x^{1}$, meaning that $y^{1}$ is the preference-midpoint between $x^{0}$ and $x^{1}$. We define $U\left(y^{j}\right)=j / 2$. With further similar refinements, $U$ can be defined to any degree of specificity. By continuity, $U$ is extended to all outcomes. Tradeoff consistency, or transitivity of $\sim$, implies, together with the other conditions, that the construction of utility does not run into contradictions. For unstance, it can be demonstrated that $y^{2 k}=x^{k}$ not only for $k=1$, but also for all $k>1$. The subjective probability of each event $A$ is the utility gained for sure that is equivalent to gaining one utility unit conditional on event $A$. Again, tradeaff consistency together with the other conditions ensures that this definition of subjective probabilities does not run into contradictions and yields an additive probability 
measure. Finally, tradeoff consistency also ensures that the expectation generated by the constructed probabilities and utilities properly evaluates acts.

\subsection{Discussion}

Jonathan Baron (personal communication) pointed out an analogy between tradeoff consistency and Savage's $\mathrm{P} 3$, a well-known monotonicity condition.

From $\left(E_{1}, \alpha ; E_{2}, x_{2} ; \ldots ; E_{n n}, x_{n 2}\right) \succcurlyeq\left(E_{1}, \beta ; E_{2}, x_{2} ; \ldots ; E_{n}, x_{n}\right)$ we can derive the preference ordering $\alpha \neq \beta$ between the outcomes. Savage's P3 requires that such derived orderings of outcomes be independent of (the nomnull) event $E_{1}$ and of the common outcomes $x_{j}$ outside of $E_{1}{ }^{4}$ Tradeoff consistency extends this independence to derived orderings of strengths of preferences. The resulting condition is, of course, considerably more restrictive, but the intuitive analogy is interesting.

Earlier versions of tradeoff consistency, used by Wakker (1989) and athers, used two *-preference orderings over strengths of preferences instead of our $\sim$, a strict one with weak preference $\succcurlyeq$ in $\mathrm{Eq} .3 .6$ and strict preference $\prec$ in Eq. 3.7, and a weak one with weak preferences $\succcurlyeq$ and $\preccurlyeq$ in those equations. Wakker (1989, Theorem IV.2.7) used absence of contradictions between these relations to characterize subjective expected utility. Theoretical follow-up papers used similar preference conditions (Abdellaoui, 2002; Bleichrodit \& Quiggin, 1999; Ebert, 2000; Gilboa, Schmeidler, \& Wakker, 2002; Karni, 2001; Prelec, 1998; Schmidt \& Zank, 2001a; Tversky \& Kahneman, 1992; Wakker \& T'versky, 1993). The version of this chapter is less restrictive and more powerful, closer to empirical applications, and simpler and more appealing because it avoids asymmetries in the definition.

Several empirical studies have used "tradeoff" reasonings as described here to meat sure utility. As we saw $\sim^{*}$ reveals identities of utility differences under expected utility. Wakker \& Demeffe (1996) elicited values $x^{0}, \ldots, x^{4}$ as described in the proof of Observation 8 , at the end of the preceding Section. Normalizing utility such that $U\left(x^{0}\right)=0$ and $U\left(x^{4}\right)=1$, implies $U\left(x^{j}\right)=j / 4$ for all $j$. We think that measurements by means of the relation $\sim^{*}$ encourage subjects to invoke rational procedures of weighing positive against negative arguments, rather than resorting to irrational noncompensatory heuristics:

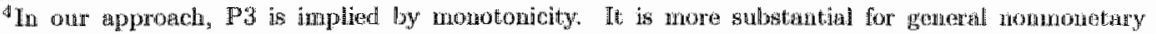
outcomes.
} 
(compare Broome, 1991, p. 7). In this manner, the relation $\sim$ is suited for constructive elicitations of preferences (Payne, Bettman, \& Schkade, 1999).

Fenneme \& van Assen (1998) used the method to investigate whether utility for losses is convex or concave. Whereas economic studies have usually assumed concavity of utility throughout, psychologists have found evidence for convex utilities for losses (Tversky \& Kalmeman , 1992). The traditional methods for measuring utility have been confounded by other aspects of risk attitude such as probability transformations. Fennema and van Assen's study avoided a number of such confoundings and found more convexity than concavity for utility in the loss domain. Other works that used the tradeoff method for measuring utility are Fennema \& van Assen (1998), Abdellaoui (2000), and Bleichrodt \& Pinto (2000). Abdelleoui \& Munier (2001) used the method in a dual manner to measure probability transformation.

Tradeoff consistency can be criticized for being based on the relation $\sim$ * which is not a primitive but is derived from the preference relation. The derivation is, however, simple, and substitution of the definition of $\sim^{*}$ in tradeoff consistency directly yields a restatement of tradeoff consistency in terms of preferences: Eqs. 3.6 and 3.7 , and

$$
\left(A_{1}, \alpha ; A_{2}, v_{2} ; \ldots ; A_{m}, v_{m}\right) \sim\left(A_{1}, \beta ; A_{2}, w_{2} ; \ldots ; A_{m}, w_{m}\right)
$$

should al ways imply"

$$
\left(A_{1}, \gamma_{i}, A_{2}, v_{2} ; \ldots ; A_{m}, v_{m}\right) \sim\left(A_{1}, \delta ; A_{2}, w_{2} ; \ldots ; A_{m n_{2}}, w_{m}\right)
$$

Tests of this preference condition naturally result from cross-checkings in quantitative utility measurements. Such tests were conducted in an informal experiment as follows, where a pair of outcomes $(x, y)$ denotes a fifty-fifty gamble yielding $x$ or $y$. First, outcomes $10,000=x^{6}, \ldots, x^{4}$ were elicited such that $\left(1000, x^{j}\right) \sim\left(8000, x^{j-1}\right)$ for $j=1, \ldots, 4$, implying $\left[x^{j} ; x^{j-1}\right] \sim \sim^{*}\left\{x^{1} ; x^{0}\right]$ for all $j$. Second, after some intermediate questions to redkce memory effects, the experimenter chose a $g>x^{4}$ and $G, y^{2}, \ldots, y^{4}$ were elicited such that $\left(g, y^{j}\right) \sim\left(G, y^{j-1}\right)$ for $y=1$ to 4 , where we set $y^{0}=x^{0}$ and $y^{1}=x^{1}$. Tradeoff consistency requires that $y^{j}=x^{j}$ also for $j=2, \ldots, 4$. The null lypothesis of these equatities was not rejected. The discrepancies between the $y^{j}$ 's and $x^{j}$ 's were smaller then discrepancies caused by some framing effects that were also tested and that were significant. Such findings, if confirmed by thorough experiments, suggest that deviations 
from expected utility primarily concern basic rationality conditions such as transitivity and framing invariance, and to a lesser extent sophisticated conditions such as tradeoff consistency, the sure-thing principle, etc.

\subsection{Conclusion}

The relation $\sim *$, introduced in this chapter, generalizes and simplifies earlier preference foundations for expected utility based on the tradeoff technique. It provides a unifying tool for quantitative empirical elicitations, qualitative axiomatic testing, and theoretical derivations of expected utility. Extensions to nonexpected utility models will be provided by Köbberling \& Wakker (2002b, see Chapter 4 ). We hope that the $\sim^{*}$ relation, while formally revealing risky utility differences, nevertheless appeals to an intuition of strength of preference and thereby can help to relate risky and riskless utility (Abdellaoui, Barrios, \&. Wakker, 2002). In general, we hope that one concept of cardinal utility, relevant for risky decisions, intertemporal decisions, welfare evaluations, and central in all social sciences, will emerge from current decision theories, an index of goodness in the sense of Bentham. (1789), Broome (1991), or Robson (2001). 


\section{Chapter 4}

\section{Preference Foundations for}

\section{Nonexpected Utility: A Generalized and Simplified Technique}

This chapter examines a tradeoff-consistency technique for testing and axiomatically foundung decision models. The techmique modifies earlier tradeoff-consistency techniques by only considering indifferences, not strict preferences. The resulting foundations are, at a time, more general and more accessible than earlier results, regarding both the technical and the intuitive axioms. The technique is applied to three popular theories of individual decision under uncertainty and risk, i.e. expected utility, Choquet expected utility, and prospect theory. The conditions used are better suited for empirical measurements of utility than earlier conditions, and accordingly are easier to test. ${ }^{1}$

\subsection{Introduction}

This chapter presents preference foundations for expected utility, Chocuet expected utility, and prospect theory. Our technique can be applied to any model based on weighted averages, such as intertemporal choices with discounted utility (Gilboa, 1989), utilitarian welfare (Harsany, 1955), and their rank-and sign-dependent generalizatiors. We will formulate our results for decision under risk and uncertainty.

Many preference foundations have been proposed before. Such foundations typically

\footnotetext{
"The rosults in this chapter were first formulated in (Koblerling \& Wakker, 2002b).
} 


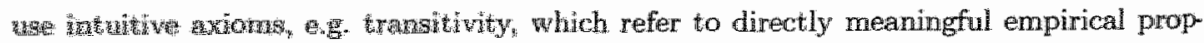

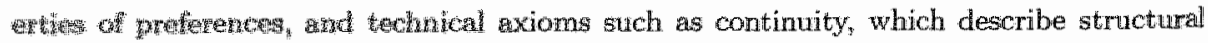
we a

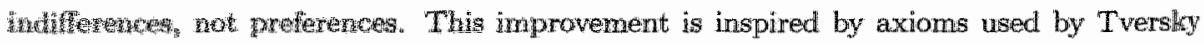

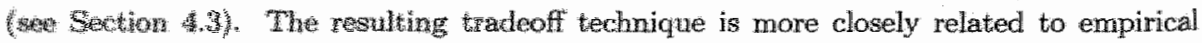

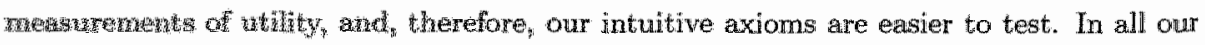

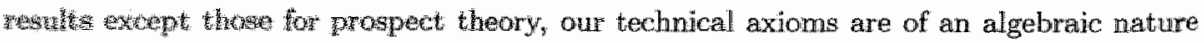

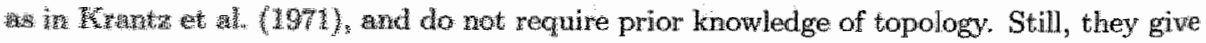

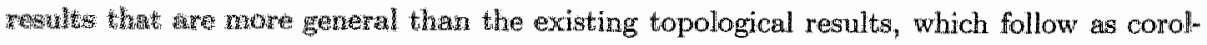

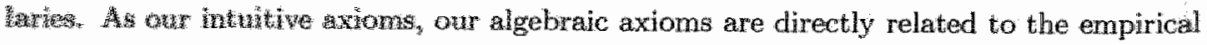

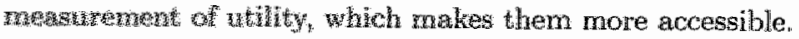

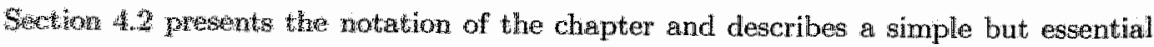

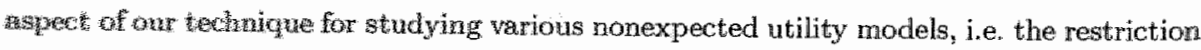

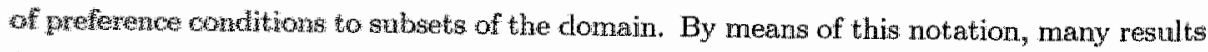

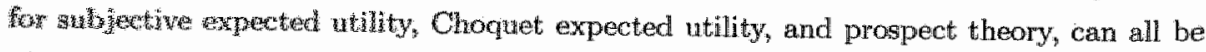

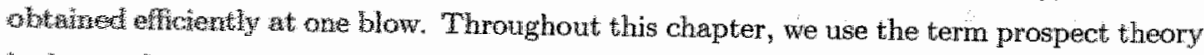

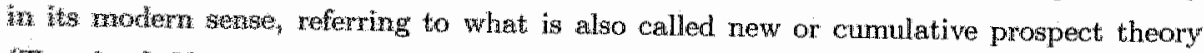

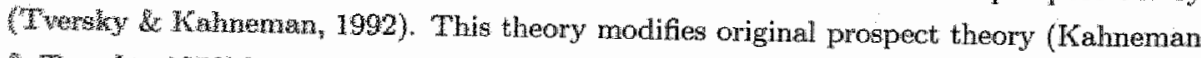
(1: Twersky, 1979) by incorporating Quiggin's (1981) and Schmeidier's (1989) ideas of rank-

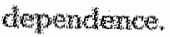

Section 4.3 describes the indifference-tradeoff technique. Section 4.4 is the central sectron of wis chepter, with preference foundations for subjective expected utility in Subsec-

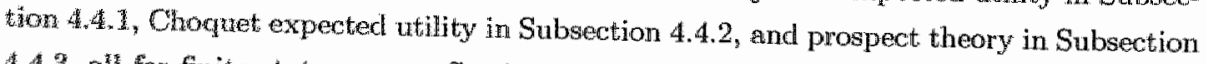
4.4.3, the finite state spaces. Section 4.5 describes extensions to infinite state spaces, wo-butwome arts, and decision under risk, and conditions for studying the curvature of utility. Section $4: 6$ presents a detalled comparison with the existing literature. Most preference axiomatizations hawe assumed rich outcome spaces. For such outcome spaces, our results are the most general in the sense that for each existing axiomatization, the axfoms used imply our axioms but not vice versa. Siection 4.7 concludes. Appendix A of this chapter elaborates on the way in which our techniques are more general than earlier tecliniques, with details about enrlier tradeoff-consistency axioms deferred to Appendix $B$. Proofs are in Appendix C. 


\subsection{Basic Definitions of Decision under Uncertainty, and their Restrictions to Subsets of the Domain}

We use Savage's (1954) model for decision under uncertainty, with $S=\{1, \ldots, n\}$ the state space and $X$ the outcome space. For simplicity, the state space is taken finite. Subsection 4.5.1 describes extensions to general state spaces. In the derivations of expected utility and Choquet expected utility (Subsections 4.4 .1 and 4.4.2), richness is imposed on $X$ through a solvability axiom. This axiom can be satisfied if $X$ is an interval in the reals, designating money, or $I R_{+}^{m s}$, designating commodity bundles. It can also be satisfied for particular finite ("equally-spaced") sets of, sey, discrete and qualitative health states. In the derivation of prospect theory (Subsection 4.43), a more restrictive topological condition, namely connectedness, is imposed on $X$, which includes intervals in the reals and $\mathbb{R}_{+*}^{m}$, but no finite sets.

$X^{n}$ is the set of acts. An act $f=\left(f_{1}, \ldots, f_{n}\right)$ is identified with the function assigning $f_{j}$ to each state $j$. Outcomes are identified with constant acts. A preference relation $\succcurlyeq$ is a binary relation on $X^{n}$. Strict preference $\succ$, indiffenence or equivalence $\sim$, and reversed preferences $\preccurlyeq$ and $\prec$, are defined as usual. Given that outcomes are identified with constant acts, preferences also apply to outcomes.

A function $V$ represerts $\succcurlyeq$ on a set $F \subset X^{n}$ if

for all acts $f, g \in F, f: g$ if and only if $V(f) \geq V(g)$.

In this definition, we implicitly assume that $F$ is contained in the domain of $V$, and that $V$ maps acts to the reals. Restrictions of representations and preference conditions to subsets of acts, such as $F$ above, play a central role in this chapter. In the above definition, as in other definitions throughout this chapter, we omit "on $F^{\prime \prime}$ if $F=X^{n}$. If a representing function exists (i.e., on $\left.X^{n}\right)$, then $\succcurlyeq$ is a weak order, meaning that it is complete $(f \geqslant g$ or $f \preccurlyeq g$ for all acts $f, g$ ) and transitive. In all models in this chapter, weak monotonicaty will hold, i,e. if $f_{j} \succcurlyeq g_{j}$ for all $j$ then $f \succcurlyeq g$. 
5ubjective expected utilty (SEU) holds on a set $F \subset X^{n}$ if there exist nomnegatina probabilities $p_{1, \ldots,} p_{n}$ summing to one and a utilty function $U: X \rightarrow I R$ such that

$$
S E U^{r}: f \rightarrow \sum_{j=1}^{n} p_{j} U\left(f_{j}\right)
$$

represents preferences on $F$. We then call $F$ an $S E U$-set.

For an outcome $\alpha_{1}$ an act $f_{3}$ and a state $f_{3}, \alpha_{i} f$ denotes the act $f$ with $f_{i}$ replaced by $\alpha$. A state $z$ is $r u l l$ on $F \subset X^{n}$ if $\alpha_{i} \sim f$ for all $\alpha_{i} f f$ in $F$. Otherwise the state nonnul on $F$. If a state is nonnull on an SEU-set $F$, then its probability must be positive. The converse implication holds under minimal richness of $F$ (if $F$ contains $\alpha_{i} f$, f with $\left.U(\alpha)>U\left(f_{i}\right)\right)$. Finally, $\succcurlyeq$ is trivial if all acts are indifferent.

\subsection{A General Tool for Axiomatizing Models}

The following $\sim^{*}$ relation is central in this chapter. It can be interpreted as a strength of preference relation over outcomes revealed from ordinal preferences over acts. For $F \subset X^{\mathrm{m}}$ and ontcomes $\alpha, \beta, \gamma, \delta$, we write

$$
[\alpha ; \beta] \sim \sim_{F}^{*}[\gamma ; \delta] \text { or } \alpha \beta \sim \sim_{F}^{*} \gamma \delta
$$

if there exist acts $f . g$ and a state $j$ that is nommull on $F$, such that

$$
\alpha_{j} f \sim \beta_{j} g \text { and } \gamma_{j} f \sim \delta_{j} g
$$

with all four displayed acts contaimed in $F$. The interpretation is that receiving $\alpha$ instead of $\beta$ apparently does the same as receiving $\gamma$ instead of $\delta, i$ exactly offset the joint receipt

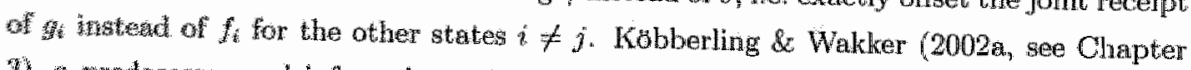

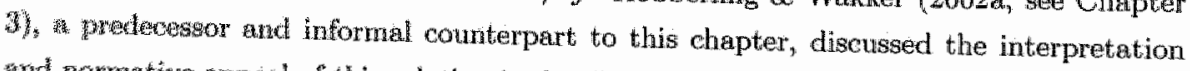

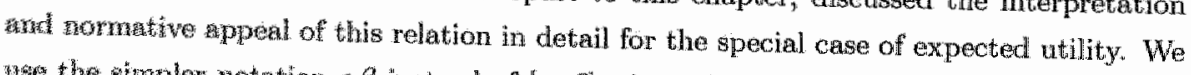

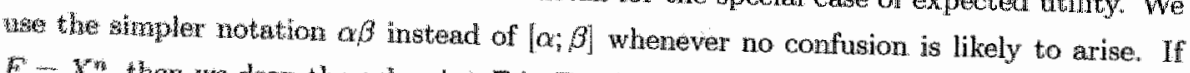
$F=X^{*}$, then wo drop the stibscript $F$ in Eq. 4.1 and write $\cdots$. A similar relation, where mtax wat ond cimension are matched whenever they match the same interval on another dumsion, defined by Tversky (1977, appendix invariance axiom). Substitution shows 
that, for SEU-sets $F$,

$$
\alpha \beta \sim_{F}^{*} \gamma \delta \Rightarrow U(\alpha)-U(\beta)=U(\gamma)-U(\delta)
$$

We next list some symmetries for the relation $\sim_{F}^{*}$ that correspond with the interpretation as derived strength of preference.

Observation 9 If $\succcurlyeq$ is a weak onder, then the following four relations ane equivalent:

(i) $\alpha \beta \sim_{F}^{*} \gamma \delta$;

(ii) $\gamma \delta \sim \sim_{F}^{*} \alpha \beta$;

(iai) $\beta \alpha \sim \sim_{F}^{*} \delta \gamma$;

(iv) $\delta \gamma \sim_{F}^{*} \beta \alpha$.

The second symmetry follows from the first by reversing the right-hand and left-hand indifferences in Eq. 4.2, the third from reversing the right-hand and left-hand acts in both indifferences in Eq. 4.2, and the fourth by combining these two operations. The $\sim_{F}^{*}$ relation need not be transitive and, consequently, need not be an equivalence relation. We will see in Observation 34 that transitivity is a strong restriction that comes close to implying SEU on the relevant set $F$.

Eq. 4.3 shows that the $\sim_{F}^{*}$ relation can be used to elicit equalities of utility differences, which, for cardinal utility, suffices to determine the entire utility function. Obviously, we would want such measurements to be consistent, and not lead to contradictions. Therefore, there should not exist one pair of acts $f, g$ and a nonmull state $j$ that reveal $\alpha \beta \beta \sim F$ as in Eq. 4.3, and another pair of acts $f^{\prime}, g^{\prime}$ and a nonnull state $j^{\prime}$ that imply $\alpha^{\prime} \beta \sim_{F^{\prime}}^{*} \gamma \delta$ for an $\alpha^{\prime} \nsim \alpha$. Such a finding would not combine well. with the intuition that, e.g., $\alpha^{\prime} \beta$ should exceed $\alpha \beta$ as soon as $\alpha^{\prime} \succ \alpha$, and, therefore, not both should match the same $\gamma \delta$. Substitution of SEU shows that the two $\sim_{F}^{*}$ relations require the equallity $U(\alpha)=U\left(\alpha^{\prime}\right)$, indeed. Similarly (and logically equivalent by Observation 9), if any one of the four outcomes in a $\sim_{F}^{*}$ relation is replaced by a nonequivalent outcome, then the $\sim_{F}^{*}$ relation should not hold any more. This requirement is called trudeoff consistency on $F$.

Preference conditions similar to tradeoff consistency can be found in some early papers co-authored by Amos Tversky (Krantz et all, 1971, Section 6.11.2, standard-sequence 
invariance; Tversky, 1977, appendix, invariance; Tveraky \& Kahmemam, 1991, reference interlocking; Tversky, Sattath, \& Slovic, 1988, the interlocking condition), and recently also in Myramoto (1988, Definition 7.10; 1999, Definition 12) and Skiadas (1997, Axiom A10). All of these papers used additional conditions to ensure the existence of additue representations, with their tradeof conditions serwing only to imply proportionality across different dimensions. Such proportionality is also "automatically" implied by conditions that characterize parametric families of utility, or multiattribute decomposability (Zank, 2001).

The tradeoff-consistency conditions are formulated in terms of $\sim$-relations, which are not empirical primitives, but are derived from preferences. The condition can easily be reformulated directly in terms of preferences, through the requirement that the three indifferences $\alpha_{i} f \sim \beta_{i} g_{n} \gamma_{i} f \sim \delta_{i} g_{3}$ and $\alpha_{j} x \sim \beta_{j} y$, should always imply the fourth indifference $\gamma_{j} x \sim \delta_{j} y$, under restrictions corresponding to the theary under consideration. This condition is equivalent to tradeoff consistency and is analyzed in Appendix B of this: chapter. We use the $\sim_{F}^{*}$-relations in the main text because they are usefui concepts in decision making.

\subsection{Applications of our Technique}

This section shows how our tradeoff technique can be applied to three poptlar models of decision under uncertainty: subjective expected utility, Choquet expected utility, and prospect theory. Gilboa, Schmeidler, \& Wakker (2002) applied a similar technique to case-based decision theory.

\subsubsection{The Algebraic Approach for Subjective Expected Utility}

We use the notation $\alpha_{i} \rho_{j} f$ to denote the act $f$ with $f_{i}$ replaced by and $f_{j}$ replaced by $\beta$, for $i \neq y$. Empirical measurements of utility often elicit standard sequences on sets $F \subset X^{m}$, i.e. sequences of outcomes $\alpha^{0}, \alpha^{1}, \ldots$ for which there exist an act $f$, states $i \neq j$

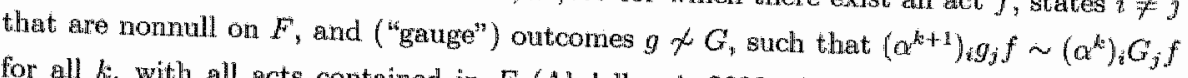
for all $k$, with all acts contained in $F$ (Abdellaoui, 2000; Abdellaoui, \& Munier, 2001; Bleiclirodt \& Pinto, 2000, 2001; Bleichrodt, Pinto, \& Wakker, 1999; Fennema \& van Assen, 1998; Wakker \& Deneffe, 1996). A standard sequence entails a special way of 
obserwing that $\alpha^{k+1} \alpha^{k} \sim_{F^{*}}^{*} \alpha^{k} \alpha^{k-1}$ for all $k$, and, thus, of obtaining a sequence of outcomes that is equally spaced in utility units on an SEU-set. The nonequivalence of the gauge outcomes $g$ and $G$ serves to avoid trivial standard sequences of equivalent outcomes. The standard sequence is increasing if $G \succ g$ and decreasing if $G \prec g$. Under monotonicity requirements, $\alpha^{k+1} \succ \alpha^{k}$ for all $k$ if the standard sequence is increasing and $\alpha^{k+1} \prec \alpha^{k}$ for all $k$ if the standard sequence is decreasing (Lemma 32). Formally, standard sequences can be finite or infinite.

The two technical axioms of the "algebraic approach" defined next are related to the method of measuring utility just described. Solvobility holds if for all acts $f, g$, outcomes $\alpha, \gamma$, and states $i$ with $\alpha_{i} f \succ g \succ \gamma_{i} f$ there exists an "intermediate" outcome $\beta$ such that $\beta_{i} f \sim g$. The condition ensures, for instance, that a further outcome $\left(\beta \Rightarrow \alpha^{k+1}\right.$ can be defined to prolong an increasing standard sequence as long as a sufficiently extreme $\gamma$ exists to have $\gamma_{i} g_{j} f>\left(\alpha^{k}\right)_{i} G_{j} f>\left(\alpha^{k}\right)_{i} g_{j} f$. In earlier papers, this condition was usually called restricted solvability. Given its importance, we prefer to use a mare efficient term.

To prepare for the second technical axiom, we make two observations. A standard sequence is bounded if there exist an outcome weakly more-preferred, and an outcome weakly less-preferred, than all elements of the standard sequence. On SEU-sets, a bounded standard sequence must be finite. Otherwise, the standard sequence being equally spaced in utility units, either the utility of the more-preferred outcome would have to be infinite if the standard secuence were increasing, or the utility of the less preferred outcome would have to be minus infinite if the standard sequence were decreasing. In each case, SEU, which does not allow for infinite utility, would be violated.

The second abservation concerns the degenenate case where exactly one state is nonnull. Then preferences do not reveal uncertainty. There do not exist nontrivial standard sequences, and finiteness of the bounded standard sequences is vacuously satisfied. To avoid non-Archimedean representations, we then involie a condition from represertation theory for single-dimensional objects. It is well known that there exists a real-valued representation for a weak order on the set of outcomes if ard only if there exists a courtable onder-dense set of outcomes, i.e. a set of outcomes such that for all outcomes $\alpha, y$ with $\alpha \succ \gamma$ there exists an element $\beta$ of the order-dense set with $\alpha \succcurlyeq \beta \succcurlyeq \gamma$ (Krantz et al, 1971, Theonem 2.2). The Anchimedean axiom holds on $F$ if every bounded standand sequance is finite, and in the degenerate case of exactly one nonnull state on $F$ there exists a countable order-dense set of outcomes. 
The main axiom characterizing SEU is tradeoff consistency, which means that tradeoff consistency holds on $X^{n}$, the whole set of acts. At this point, we are ready to state the first representation theorem, which characterizes SEU.

Theorem 10 /Characterization of SEU] Assume that solvability holds. The following two statements are equivalent for $\geqslant$ on $X^{n}$.

(i) SEU holds.

(ii) $\succcurlyeq$ satisfies

(a) weak ordering;

(b) weak monotonicity;

(c) the Archimedean axiom;

(d) tradeoff consistency.

Observation 11 /Uniqueness Results for Theonem 10/ Suppose that the assumptions and staternents of Theorem 10 hold.

(a) In the trivial case where all states are null, utility is constant and the probabilities can be chosen arbitrarily.

(b) In the degenerate case of exactly one nonnull state, utility is unique up to a strictly increasing transformation. The probability of the nonrull state is one, and all other states have probability zero.

(c) In the nondegenerate case of two or more nonnull states, utility is unique up to unit and location, and the probabilities ane uniquely determined.

The only nonnecessary condition in the above theorem, and in some results given later, is solvability. Examples in Appendix A of this chapter will further illustrate to what extent solvability is restrictive. Theorem 10, as several of its variations in the literature, can be interpreted as a modification of Savage's (1954) SEU characterization that allows for finite state spaces, or as a modification of Anscombe \& Aumann's (1963) SEU characterization to the case where outcomes need not be lotteries oxer prizes to be evaluated linearly through an expected utility formula, and where multistage gambles and assumptions on dynamic optimization need not be invoked. The latter modification is especially desirable 
for the nonexpected utility models derived in later sections. The main motivation for Wakker (1984) to develop a predecessor of our result was yet another, namely that it can be interpreted as an extension of de Finetti's book-making argument, in the sense of subjective expected value maximization, to nonlinear utility.

An interesting alternative characterization of SEU results if tradeoff consistency in (d) in Statement (ii) of Theonem 10 is replaced by transitivity of $\sim$, or by the requirement that $\sim$ is an equivalence relation (Observation 34). The adaptation of these results to nonexpected utility models is not very transparent. It can be seen that the $\sim^{*}$ relation. need no more be complete for such models, so that complex acyclioity conditions have to be invoked instead of transitivity. We do not elaborate on this poimt, and only consider tradeoff consistency in the main text.

\subsubsection{The Algebraic Approach for Choquet Expected Utility}

We next turn to Choquet expected utility; the rank-dependent generalization of SEU. Primarily, it allows for nonadditive probabilities. A weighting function $W$ is a set function $W: 2^{S} \rightarrow[0,1]$ such that $W(0)=0, W(S)=1$, and $A \subset B \Rightarrow W(A) \leq W(B)$. Other terms are capacity or nonadditive probability. Choquet expected utilty (CEU) holds if there exist a weighting function $W: 2^{S} \rightarrow[0,1]$ and a utility function $U: X \rightarrow I R$ such thet

$$
C E U: f \rightarrow \sum_{j=1}^{n} \pi_{p_{j}} U\left(f_{p_{j}}\right)
$$

represents preferences. The decision weights $\pi_{\rho_{j}}$ are nomegative and sum to one. They are derived from $W$ and the act $f$ as follows:

- choose a rank-andering permutetion $p$ on $\{1, \ldots, n\}$ such that $f_{p_{1}} \ldots f_{p_{9}}$;

- defime $\pi_{\rho_{j}}=W\left(\rho_{1}, \ldots, \rho_{j}\right)-W\left(\rho_{1}, \ldots, \rho_{j-1}\right)$ for all $j ;$ in particular, $\pi_{p_{1}}=W\left(\rho_{1}\right)$.

The decision weights $\pi_{p,}$ depend on the act $f$ through the rank-ordering $\rho$, a dependency that is not expressed in the notation.

The $n$ ! sets $F \subset X^{n}$ of the form $\left\{f \in X^{n}: f_{p(n)} \nsucc \ldots\right.$. $\left.f_{p(n)}\right\}$, for some rank-ordering permutation $\rho$, are called comoncomes. A set of acts is comonotonic if it is a subset of 
a comoncone. Comoncones are maximal comonotonic sets; they are cones when mapped into utility units. The following result is immediate.

Observation 12 If CEU holds, then each comoncone is an SEU-set, with decision weights as probibilities.

Many results of CEU are obtained by applying SEU techniques within comoncones. The comonotonic degenerate case halds if on every comoncone exactly one state is nonnull. The comonotonic Archimedean axiom holds if every bounded standard sequence on $F$ is finite for wery comoncone $F$, and if, further, there exists a countable order-dense subset of $X$ in the comonotomic degenerate case. We write $\alpha \beta \sim_{c}^{*} \gamma \delta$ if there exists a comoncone $F$ such that $\alpha \beta \sim_{F}^{*} \gamma \delta$. The symmetries of Observation 9 also hold for $\sim_{c}^{*}$ instead of $\sim_{F}^{*}$. Comonotonic tradeoff consistency holds if replacing one of the four outcomes in a $\sim_{c}^{*}$ rellation by an nonequivalent outcome implies that the $\sim_{c}^{*}$ relation does not hold any more. This condition is slightly stronger than tradeoff consistency on every comoncone because it compares *-relations elicited from different comoncones. This reflects the restriction that the utility function should be the same on all comoncones. Figure 1 in Wakker \& Tversky (1993) demonstrates, for two nonnull states, that tradeoff consistency within each comoncone is too weak to characterize $\mathrm{CEU}$, so that indeed a stronger condition is required.

Theorem 13 /Characterization of CEU/ Assume solvability. The following two statements are equivalent for $\geqslant$ on $X^{n}$.

(i) CEU holds.

(ii) $\succcurlyeq$ satisfies

(a) weak ondering;

(b) weak monotonicity;

(c) the comonotonic Archimedean axiom;

(d) comonotonic tradeoff consistency.

Observation 14 [Uniqueness Results for Theorem 13] Suppose that the assumptions and statements of Theorem 19 hold.

(a) In the (trivial) case where at least one comoncone has only null states, all comoncones have only null states, the preference nelation is trivin, and utility is constant and the weighting furction can be chosen arbitrarily. 
(b) In the degenerate case of exactiy one nommill state for each comoncone, whilty is antique up to a strictly increasing thansformation, and the wetghting function is uniquely determined (taking only the values zero and one) by the requirement that on each comoncane the decision weight of the nonnull state is ane and of all other states it is zero.

(c) In the nondegenerate case whene at least one comoncone has two or more ronnull states, winty is unique up to unit and location, and the weyhting function is unguely determined.

\subsubsection{The Topological Approach for Prospect Theory}

This subsection assumes further, topological, structure (Mendelson, 1962). All the following topalogical conditions are satisfied under the common assumptions of $X$ being an interval in the reals or $X=I_{4}^{m}$. Readers not interested in general topology can simply assime any of these cases and ignore the following topological assumptions. First, we assume that $X$ is a connected topological space and that the set of acts is endowed with the product topology. For the degenerate cases, we will further assume topological separability. Appendix $\mathrm{A}$ of this chapter will demonstrate that the topological assumptions are more restrictive than the algebraic assumptions of the preceding sections, because the continuity conditions imply solvability and the Archimedean axioms.

Contimuity with respect to a connected product topology holds if and only if it holds for $X$ endowed with the order topology. Some results in Luce (2000) and Luce \& Fishburn (1991) used an equiwalent "Dedekind completeness" condition. Before turning to prospect theory, we list a corollary of our earlier results. It characterizes continuous SEU and CEU. The only nontrivial aspect that does not follow immediately from the algebraic results and Appendix $A$, is continuity of the witity functions.

Corollary 15 /Chanacterization of continwous SEV (CEU)/Assume that $X$ is a connected topalogical space and that $X^{n}$ is endowed with the product topology. For the degenerate case of exactly one nommill state (on every comoncone), assume that $X$ is also topologicalby sepanable. The following two statements are equivalent for $\succcurlyeq$ on $X^{n}$.

(1) SEU (CEU) holds with a continuous utility function. 
(it) $\succcurlyeq$ satísfies

(a) weak ordering;

(b) weak monotonucitys:

(c) continuity;

(d) (comonotonic) tradeoff consistencys.

Uniqueness reaults are as in Observation 11 (Observation 14), with anly one modification: In the degenerate case, wility is unique ap to strictly increasing transformations that furthermare have to be continuous.

For prospect theory, an additional structural assumption is added. It is assumed that the outcome set contains a special element, denoted $r$, and called the reference point. Gains are outcomes strictly preferred to $r$, losses are outcomes strictly less-preferred than $r$. For an act $f$ we define the act $f^{+}$by $f_{j}^{+}=f_{j}$ if $f_{j} \geqslant r$ and $f_{j}^{+}=r$ otherwise, and the act $f^{-}$by $f_{j}^{-}=f_{j}$ if $f_{j} \leqslant r$ and $f_{j}^{-}=r$ otherwise.

(Cumulative) prospect theory (CPT) holds if there exist weighting functions $W^{+}, W^{-}$: $2^{S} \rightarrow[0,1]$, and a utility function $U: X \rightarrow \mathbb{R}$ with $U(r)=0$ such that

$$
C P T(f)=C P T^{+}\left(f^{+}\right)+C P T^{-}\left(f^{-}\right)
$$

represents preferences. Here $C P T^{+1}$ denotes Choquet expected utility with respect to $\mathrm{W}^{+{ }^{+}}$, and $C P T^{-}$denotes Choquet expected utility with respect to the dual of $W^{-}$, defined by $A \mapsto 1-W \sim(S-A)$.

The way of integration of prospect theory, symmetric about zero, is reminiscent of the Śpos (1979) integral, which is the special case of $W^{+}=W^{-}$. The Choquet integral is the special case in which $W^{-}$is dual to $W^{+}$. The prospect theory functional is more complex than the SEU or CEU functionals. Preliminary analyses suggest that its axiomatization for the algebraic approach is very complex, primarily beckuse "conditional certainty equivalents," used throughout the topological proofs, no more need to exist. This axiomatization currently remains as an open problem. We, therefore, consider only the topological approach.

A sign-comoncone $F$ is a subset of $X^{n}$ for which there exist a rank-ordering permutation $\rho$, and $0 \leq k \leq n$, such that $F=\left\{f \in X^{n}: f_{\rho(1)} \succcurlyeq \ldots \succcurlyeq f_{\rho(k)} \succcurlyeq r \succcurlyeq f_{\rho(k+1)} \succcurlyeq \ldots \succcurlyeq\right.$ $\left.f_{p(n)}\right\}$. Acts from $F$ yield only gains if $k=n$ and only losses if $k=0$. A set of acts is 
sign-comonotomic if it is a subset of a sign-comoncone. This holds if and only if the acts are comonotonic and, furthermore, there is no state for which one act yields a gain and another a loss.

Observation 16 If CPT holds, then each sign-comoncone is an SEU-set.

We write $\alpha \beta \sim_{s c}^{*} \gamma \delta$ if there exists a sign-comoncone $F$ such that $\alpha \beta \sim_{F}^{*} \gamma \delta$. The symmetries of Observation 9 also hold for $\sim_{s c}^{*}$. Sign-comonotonic tradeoff consistency holds if replacing one of the four outcomes in a $\sim_{g c}^{*}$ relation by a nonequivallent outcome implies that the $\sim_{s c}^{*}$ relation does not hold any more.

The taxonomy of degenerate cases for CPT is complex and space-consuming, with several combinations of gains- and loss-representations. We, therefore, restrict attention to the nondegenerate case in which genuine tradeoffs between gains and losses exist. $\succcurlyeq$ is truly mixed if there exists an act $f$ with $f^{+} \succ r$ and $f^{-} \prec r$. It is easily verified that this condition holds under CPT if and only if there exists an event $A$ such that $W^{*}(A)>0$ and $W^{-}(S-A)>0$, and the outcome set contains at least one gain and one loss.

To guarantee consistency in tradeoffs between gains and losses, one more condition is imposed. Gain-loss consistency holds if for all acts $f_{n} g$ with $f \sim r_{y} f^{+} \sim g^{++}$and $f^{-} \sim g^{-}$ we have $g \sim r$.

Theorem 17 /Characterization of continuous CPT/ Assume that $X$ is a connected topological space and that $X^{n}$ is endowed with the product topology. Let $\$$ on $X^{n}$ be truly mixed. The following two statements are equivalent.

(i) CPT holds with a continuous rutility fusction.

(ii) $\succcurlyeq$ satisfies

(a) weak ordering;

(b) weak monotonicity;

(c) continuity;

(d) gain-loss consistency;

(e) sign-comonotonic tradeoff consistency.

Observation 18 [Uniqueness Results for Theorem 17] Suppose that the assumptions and statements of Theorem 17 hold. Then the weighting functions of CPT in Statement (i) are wnique and, given $U(r)=0$, utility is unique up to unit. 


\subsection{Further Applications}

This sections sketches further applications of the tradeoff techrique.

\subsubsection{Infinite State Spaces}

So far, we have assumed that the state space is a finite set $\{1, \ldots, n\}$. In this section, we briefly explain how the results of the preceding sections can be extended to arbitrary state spaces. Writing out all extensions would take some space, and would be routine; therefore, we only describe the extensions werbally.

Assume teneral state space $S$, endowed with an algebra, possibly a sigma-algebra, whose elements are called events. An act is a finite-valued function from $S$ to $X$, where the inverse of each outcome is an event. An act is denoted by $\left(E_{1}, x_{1} ; \ldots ; E_{n,}, x_{n}\right)$, where the $E_{j}$ s are events partitioning $S$, and the act assigns outcome $x_{j}$ to each state in $E_{j}$.

The extension of the results of previous sections to this setup is obtained as follows. First, we restrict attention to a fixed partition of events $E_{1}, \ldots, E_{n}$. The corresponding set of acts is isomorphic to the suets studied in preceding sections, and here all theorems derived before can be applied. Next, the representations can be made to agree on all finite partitions for which the nondegenerate case applies and, hence, utility is unique up to

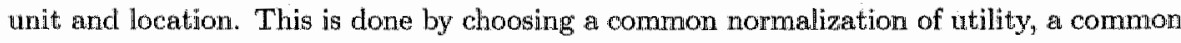
refinement for each pair of partitions, and applying the uniqueness results of preceding sections to ensure that the representations coincidle on their common domain. Because every pait of acts can be considered to be measurable with respect to a sufficiently fine partition, one representation results for the preferences between all acts.

In the preceding peragraph, the ${ }^{*}$-relations $\left(\sim^{*}, \sim_{c}^{*}\right.$; or $\left.\sim_{s c}^{*}\right)$, formally depend on the pertitions chosen, and tradeoff-consistency conditions should be related to particular par. titions. By considering common refinements of partitions, it becomes obvious that these dependencies on partitions can be dropped. Wo can define *-relation as the union of wll the conesponding partition-dependent *-relations. Tradeoff consistency can be defined with respect to the, partition-independent, *relation thus defined. Finally, extensions to acts with infinitey many outcomes can be obtained through the techniques of Wakker (1993b). 


\subsubsection{Restriction to Two-Outcome Acts}

A generalization of CEU (or any of the other models) can be obtained by imposing the model only on two-outcome acts. On this domain, the empirical evidence for CEU is most convincing, and the domain is rich enough to uniquely determine the utility function and the weighting function (Luce, 1991, Luce 2000 Chapter 3). For SEU, this point was obserwed before by Pfanzagl (1959). Ghirardato \& Marinacei (2001, 2002) used this technique to derive many applications such as definitions of ambiguity aversion. Ghirardatio et al. (2001) used it to obtain interesting axiomatizations of Bewley's (1986) incompletepreference model, and Gilboa \& Schmeidler's (1989) and Chateauneuf's (1991) multiple priors model without requiring utility to be linear in probability-mixing.

All of the above papers used restrictions of a "bisymmetry axiom," discussed further in Section 4.6, to two-outcome acts. Tradeoff consistency can be used to provide alternative characterizations of such models. Assume that all sets $F$ considered above are restricted to two-outcome acts. First, our theorems provide representations on ench two-dimensional subspace of acts constant on some given event $A$ and the complement thereof. Next, tradeoff consistency thus restricted ensures that all these two-dimensional representations, for the various events $A$, have the same utility function. Monotonicity of the capacity follows from monotonicity of preference. Thus, one CEU representation on the set of all binary acts follows. SEU and CPT can similarly be generalized to two-outcome acts.

\subsubsection{Decision under Risk}

Decision under risk can be considered a special case of decision under uncertainty (Wakker, 1990). Under decision under risk, we consider an outcome set $X$ as before. The domain of preference consists of lotteries, i.e. finite probability distributions $\left(p_{1}, x_{1} ; \ldots ; p_{i 2}, x_{n}\right)$ over $X$. These probabilities are not endogenously derived from preferences but are available as prior information about the choice objects, in the same way as the outcomes and, for uncertainty, the events are. Such probabilities are, therefore, called exogenous.

We define the unit interval $[0,1]$ as the underlying state space, with events being subintervals. Probabilities correspond to lengths of imtervals. Each act generates a lottery over the outcomes, and for eacla lottery there exist several acts that generate it. Preferences between acts are determined by preferences between the corresponding lotteries. Obviously, this implies that different acts that generate the same probability distribution over 
outcomes are indifferent.

As in Chateanneuf \& Wakker (1999, p. 144), the results of the preceding section car be used to axiomatize preferences between lotteries. Under SEU, CEU, and CPT, two intervalds of the same length, and two events with the same probability, have the same: weight in all models. Consequently, the subjective probability measure $P$ of $\mathrm{SEU}$, the

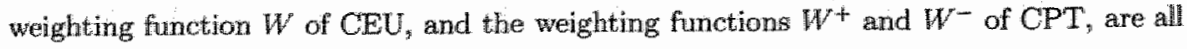
transiforms of the exogenous probabilities. By additivity and nonatomicity, $P$ of SEU must be identical to the exogenous probabilities, and a regular expected utility representation results, alternative to, and obviously less appealing than, the one of won Neumann $b$ Morgenstern (1944). The CEU model reduces to rank-dependent utility, with $W(A)=w(p)$ for $p$ the exogenous probability of $A$ and $w$ a probability transformation function. In this way, our techniques provide a tradeoff-consistency characterization of rank-dependent utility that is alternative, and we think preferable, to Wakker's (1994) Theorem 12, which was based on traditional preference-based tradeoff conditions and topological conditions.

Under CPT for risk, $W^{+}(A)=w^{+}(p)$ and $F^{-}(A)=w^{-}(p)$, with interpretations similar as for rank-dependent utility. A tradeoff-consistency characterization of CPT results that improves upon Chateauneuf \& Wakker's (1999) result for reasons like those mentioned above.

\subsubsection{Utility Curvature}

"The equivalence tradleoff techniques give convenient tools for characterizing properties of utility, alternative to earlier characterizations through tradeofe techniques. For example, assume that outcomes are monetary with continuous strictly increasing utility. Then concave utility is characterized by the requirement that $\alpha>\beta,\left[\alpha_{i}^{*} \beta\right] \sim_{F}^{*}\left[\alpha^{*}+\epsilon ; \beta+\epsilon\right]$, and $\epsilon \geq 0$ can only be if $\alpha^{\prime} \geq \alpha$, where $F$ corresponds with the theory under consideration. This condition "is alternative to Wakker (1994, Corollary 16) and Wakker \& Twersky (1993, Corollary 9.3). A utility function $U_{2}$ being more concave than $U_{1}$, for corresponding decision makers $\Rightarrow$ and $\Rightarrow 2$, $[\alpha ; \beta] \sim \sim_{1, *}^{*}[\gamma ; \delta]$ and $\left[\alpha^{\prime} ; \beta\right] \sim \sim_{2, *}^{*}\{\gamma ; \delta]$ can only be if $\alpha^{\prime} \geq \alpha$. The condition entails that $U_{2}$, when expressed in $U_{1}$ units, exhibits diminishing marginal utility. This result is altemative to Wakker (1994, Theorem 14). Schmidt (2001) used tradeoff techniques to study how wariations of the reference point affect the curvature of utility under prospect 
theory. Abdellaoui (2001) used dual versions of such tools to characterize properties of probability transformations.

\subsection{A Review of Axiomatizations of Nonexpected Utility}

This section gives a detailed review of existing preference axiomatizations, and explains how our tradeoff technique can serve to generalize these results. For other reviews of preference axiomatizations for nonexpected utility, see Fishburn (1988), Karni \& Schmeicller (1991), and Schmidt (1999). Gilboa (1987), Sarin \& Waklker (1992), and Savage (1954), used nonnecessary richness assumptions on the state space in their derivtioms of $\mathrm{CEU}$ and SEU for uncertainty. In decision under risk, such richness is naturally given through the richness of the unit probability interval. Many axiomatizations of expected utility for risk have used this richness (von Neumann \& Morgenstern, 1944; Fishburn, 1970; Fishburn, 1982 and the references therein). To the best of our knowledge, only two derivations of rank-dependent utillity, and no derivation of prospect theory, has used this richness for risk (Abdellaoui, 2001; Nakamura, 1995). There are no logical relations between these works and papers that have assumed richness of outcomes. To the latter, large, class of papers we now tum.

The only nonnecessary axiom in our characterizations of SEU and CEU is solvability. Only a few axiomatizations in decision under uncertainty and risk have used algebraic structural assumptions like we did, with a nomecessary solvability axiom, and thus reached a similar structural generality. Besides Miyamoto (1988) and Wakker (1991b, 1991c), these inchude Nakamura (1990, 1992), and Chew \& Karni (1994), and some results in Luce (2000). The latter four papers used bisymmetnic functional equations, discussed later, which require the existence of certainty equivalents for every act. Therefore, these papers used anore restrictive version of solvability, as follows. For any subse $E$ of the state space, including the case where $E$ is the whole state space, there must exist an outcome $\beta$ for ench $\alpha_{E} f \succ g \succ \gamma_{E} f$ such that $\beta_{E} f m g$. We imposed this condition only for singleton events $E=\{j\}$. Example 22.c in Appendix A will show that our approach is more general. Because of their reliance on certanty equivalents, bisymmetry axioms (discussed later) become vacuous for all sign-comoncones sets that do not contain constant gain- on loss- 
acto, i.e. for wll sign-comoncones that contain mixed acts. Therefore, bisymmetry axioms cannot be irvoked in derivations of prospect theory (Chew \& Tversky, 1990). In addition, thw existence of certainty equivalents rules out the nondegenerate finite cases. Miyamoto (1988), finally, considered two dimensions and one comoncone. He excluded fnite sets through his "densely spaced" Axiom 7.9 ( $\mathrm{p}, 369)$, but other than that used solvability as general as we do.

All other axiomatizations that we are aware of in the richness-of-outcomes approach assumed connected topological spaces, or special cases thereof. The algebraic results of this chapter for Choquet expected utility and rank-dependent utility are therefore, in a. structural sense, the most general presently available. The conditions of our theorems are satisfied in every other axiomatization, but, conversely, for every ot her axiomatization some eassumption need not be satisfied in our resinlts. Within the topological approach, Corollary 15 is the most general presently available, because it does not assume topological separability except for degenarate cases. Several other papers have assumed topological separability: for the nondegenerate case (Chateauneuf, 1999, Remark 4; Quiggin, 1982, through Axioms 3 and R.2; Ghirardato \& Marinacci, 2001, 2002). Many papers assumed, more restrictively; real-valued outcomes and continuity of utility (Chew, 1989; Gul, 1992; Prelec, 1998; Segal, 1989, 1993a, 1993b; Wakker, 1984) or even linearity of utility (Chateauneuf, 1991; de Finetti, 1937; Schmidt \& Zank, 2001b; Yami, 1987; Weymark, 1981, Theorem 3). For" prospect theony, all characterizations known to us have assumed connected topological outcome spaces, often with Euclidean structures added. Bleichrodt \& Miyamoto (2002) start from more general essumptions, but derive continuliby with respect to a connected product-order topology.

Many papers have used the approach of Anscombe \& Aumann (1963), where the outcome set consists of probability distributions over prizes and preferences over outcomes are governed by expected utility (Schmeidler, 1989). In a mathematical sense, these assumptions anount to the outcome space being a convex subset of a linear space and utility being linear. Again, these assumptions are nore restrictive than continuity with respect to a connected topology. Wakker \& Zank (1999) show how SEU-axiomatizations for lin. ear ntility (Anscombe \& Aumann, 1963; de Finetit, 1937), can be derived as corollaries from results for preferencetradeoff consistency. By Proposition 30, they also follow as corollaries from the results in this chapter.

We next turm to the intuitive axions used, i.e. the axions other than continuity with 
respect to a comnected topology, solvability, and the Archimedean axiom. All axionatizations that used tradeoff consistency so far, have used a preference version as discussed in Appendix A. All these results can be generalized by means of the indifference tradeoffconsistency conditions of this chapter. Examples are: Abdellaoui (2001), Bleichrodt \& Miyamoto (2000), Bleichrodt \& Quiggin (1997, 1999), Ebert (2000), Karni (2001), Prelec (1998, Appendix. 1), LeRoy \& Werner (2000, Section 8.6), Schmidt (2001), Schmid \& Zank (2001a), Tversky \& Kahneman (1992).

Another kind of intuitive axiom has often been used to obtain weighted averages of a general outcome-transformation function, besides tradeoff consistency, namely axioms based on bisymmetry. An example of a bisymmetric functional equation is

$$
G(H(\alpha, \beta), H(\gamma, \delta))=H(G(\alpha, \gamma), G(\beta, \delta))
$$

Under a reflexiveness normalization $(G(\alpha, \alpha)=H(\alpha, \alpha)=\alpha)$, and some regularity comditions, this implies that there exists a function $U, 0<p<1$, and $0<q<1$, such that $G(\alpha, \beta)$ is the $U$-inverse of $p U(\alpha)+(1-p) U(\beta)$ and $H(\alpha, \beta)$ is the $U$-inverse of $q U(\alpha)+(1-q) U(\beta)$. If we consider decision under uncertainty with $A$ and $B$ two events, $G(\alpha, \beta)$ the certainty equivalent of the act yielding $\alpha$ for $A$ and $\beta$ otherwise, and $H$ related to event $B$ simillarly, then the bisymmetry equation leads to a preference axiomatization of SEU functionals. An appealing intuitive justification exists if the events can be interpreted as repeatable mutually independent events, as in Luce's works (Luce, 1988, Eqs. 22 and 23; Luce, 1998, "event commutativity"). Also for one-shot-event models, some authors have alluded to this interpretation (Ghirardato et Al, 2001; Pfanzegl, 1959, p. 288; Segal, $1993 \mathrm{~b}$ "order indifference"). The axiom can be naturally extended to more than two outcomes (Chew, 1989; Munnich, Maksa, \& Mokken, 2000; Nakamura, 1992, 1995; Quiggin, 1982, Axiom 4). Gul (1992) introduced a particularly appealing version of this axion that resembles the independence preference-condition of won Neumann \& Morgenstern (194it); see Chew \& Karni (1994). Ghirardato \& Marinacci (2001, 2002) and Ghirardato et al. (2001) used this kind of axiom. Wakker \& Zank (1999, Lemma 12) demonstrated that the bisymmetry axiom implies tradeoff consistency for the special case above of $F=G$, i.e. for state spaces with only two states. Therefore, the bisymmetry results can be obtained as corollaries of the tradeoff-consistency results for this special case. For general state spaces, no simple logical relations between bisymmetry and tradeoff consistency are known to us. 
Besides the greater structural generality that can be obtained with the tradeoff approach, the possibility of this approach to handle sigm-dependence, and the natural rolle of this approach in empirical measurements, we mention two other reasons why we prefer this approach to the bisymmetry approach. First, we think that tradeoffs are a natural concept in deliberations about decisions, and, therefore, we find it natural to put them central in theoretical and empirical analyses. Second, the bisymmetry axioms invoke many certainty equivalents, which complicates their empirical testing. For criticisms of tradeoff consistency, see Luce (2000, Sections 3.7.3 and 5.3.1.1).

\subsection{Conclusion}

This chapter has presented an improved technique for deriving preference foundations for decision models based on weighted averages, and their rank- and sign-dependent generalizations. We formulated our results for decision under risk and uncertainty, but they apply as well to the measurement of inequality and welfare, and intertemporal decisions. Our results are at a time more accessible and more general than earlier results, and this claim holds both for the intuitive and for the technical axioms used.

\subsection{Appendix A: The Generality of our Approach}

This appendix explains how our approach generalizes and simplifies earlier approaches.

The Generality of Thadeoff Consistency. Many axiomatizations in the literature have adopted different versions of tradeoff consistency, using preferences instead of indifferences ("preference-tradeoff consistency"). These conditions: are as follows: No four preferences should exist of the form

$$
\alpha_{i} f=\beta_{i} g, \gamma_{i} f \leqslant \delta_{i} g, \alpha_{j} v \preccurlyeq \beta_{j} w, \gamma_{j} v \succ \delta_{j} w,
$$

whth nonwall, and the acts of the second two preferences contained in a set $F^{\prime} \subset X^{\mathrm{v}}$ on which gtates $j$ is nommul. For pnefenence-tradeoff consistency, $F=F^{t}=X^{n}$, for comonotonic preference-tradeoff consistency $F$ and $F^{r}$ are required to be comoncones, and for sign- 
comonotonic preference-tradeoff consistency $F$ and $F^{t}$ are required to be sign-comoncones. Some papers used the notation $\alpha \beta \succcurlyeq * \gamma \delta$ for the first two preferences in $\mathrm{Eq} .4 .4$, and the notation $\gamma \delta \succ^{* *} \alpha \beta$ for the latter two preferences.

Wakker (1984) introduced this kind of conditions, primarily for the purpose of normative defenses of expected utility. Wakker (1989, endnote rf13 on p. 168) refers to earlier similar ideas, in particular standard sequence invariance of Krantz et al. (1971). As argued by Köbberling \& Wakker (2002a, see Chapter 3 ) in a normative defense of expected utility, we now prefer the indiflerence-tradeoff consistencies. They are more transparent and yield more general mathematical results. The basic reason is that assuming indifferences, as done in our conditions, is more restrictive than assuming preferences. More restrictive premises lead to less restrictive axioms and more general theorems.

Proposition 19 Under weak ondering,

- preference-tradeolf consistency implies weak monotonicity and trudeoff consistency,

- comonotonic prejerence-tradeoff consistency implies weak monotonicity and comonotonic tradeoff consistency, and

- sign-comonotonic preference-tradeoff consistency implies weak monotonicity and signcomonotonic tradeoff consistency.

The proposition is elementary in the sense that it only invokes weak ordering and no technical richness assumptions such as solvability or continuity. Results using the earlier versions of tradeoff consistency can, therefore, be obtained as elementary corollaries of the results based on the tradeoff consistencies of this chapter. Under weak ordering, any set of data (finite in practice) that reveals a violation of our indifference versions of tradeoff consistency, automatically implies a violation of the preference versions of tradeoff consistency. The reversed implication need not hold (Example 23 in Appendix B). The Generality of the Algebraic Approach. Luce et al. (1990, p. 49, 1. 10), and Wakker (1988, 1989 end of Section III.6) argued that the algebraic approach is preferable to the topological approach. Most of Wakker's papers used the topological approach because it stays claser to the common conventions in the economic literature.

We first state two lemmas demonstrating that the topological assumptions imply the algebraic assumptions, so that the latter lead to more general results. Then we give some 
examples where the algebraic approach applies but the topological does not, dernonstrating that the former approach leads to strictly more general results.

Lemma 20 Assume that $\succcurlyeq$ is a continuous weak onder urth respect to a connected product topology. Then solvability holds.

In the next lemmat, we a monotonicity condition that is slightly stronger than the one defined in the main text. It is, however, implied by the other conditions in all of our main theorems, as we will see later (Lemma 26). Strong monotonicity holds on $F \subset X^{\text {m }}$ if $f_{i}$ for all $f, g \in F, f>g$ whenever $f_{j} \succcurlyeq g_{j}$ for all $j$ and $f_{j} \succ g_{j}$ for a state $j$ that is nomarll on $F$. Comonotonic strong monotonicity holds if strong monotonicity holds on each comoncone, holds oration sign-comoncone.

Lemma 21 Aswume that is a continuous weak order with respect to a connected product topolomy on $X^{*}$, that $X$ is topoloyically separable in the degenerate case, and that weak and

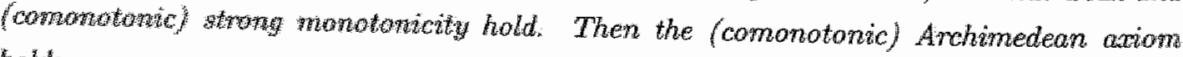
holltis.

The following examples modify examples of Krantz et al. (1971, Sections 4.8 and 6.11.1)

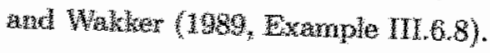

Example 22 Asstzme that $n=2$, and that SEU holds with probabilities 0.5 for each state and when the thentity function as atility. Consider the following three cases.

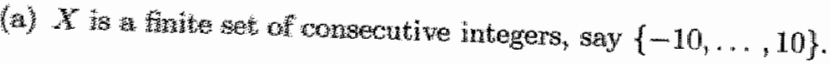

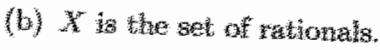
(c) $X$ contains all real numbers of the form $i+j \sqrt{2}$ with $i$ and $j$, possibly negative,
integers.

Solvability easily follows in each case because $X$ is always closed under addition and subtraction. In each case, $\geqslant$ is not continuous with respect to a connected product topology: Because $\sqrt{3}$ is not contained in any of the sets $X$, the sets $\{\alpha \in X: \alpha>\sqrt{3}\}$ and $\{\alpha \in X: \alpha<\sqrt{3}\}$ constitute a nontrivial partition of $X$ into sets that are open with 
respect to the order topology. Neither the order topology nor any other topology for which $\succcurlyeq$ is contimuous on $X$ can be connected.

In Example (c), there do not exist certainty equivalents for each act, i.e. outcomes equivalent to the act. For the act $(0, \sqrt{2})$, the certainty equivalent would have to be $\sqrt{2} / 2$, but this real number is not contained in $X(i+j \sqrt{2}=\sqrt{2} / 2$ would require that $\sqrt{2}=i /(1 / 2-j)$, i.e. that $\sqrt{2}$ were rational, which it is not). Similar reasonings show that there need not exist midpoints, in terms of utility units, between each pair of outcomes ( 0 and $\sqrt{2})$ or between each pair of equivalence classes $(e . g$., those of $(0,0)$ and $(0, \sqrt{2})$ ).

Example (a) shows that our axioms can be satisfied in finite models. Such models must be equally-spaced, i.e. the outcome set consists of one standard sequence plus other outcomes equivalent to elements of the standard sequence (Krantz et al, 1971, Section 4.8; Wakker, 1991a). In this case, different comoncones, or sign-comoncones, all have the same number, say $k$, of equally-weighted nonnull states, and can only differ in which $k$ of the $n$ states are nonmull. With $X$ the set of rationals as in Example (b), all nonexpected utility models of this chapter can be applied, as long as all decision weights are rational too. Example (c) demonstrates that the common proof techmiques, which extensively use midpoints (Ghirardato et al., 2001; Vind, 1991; Wakker, 1989, p. 60) and (conditional) certainty equivalents, cannot be used in the algebraic approach. Our proofs are primarily based on the algebraic techniques of Krantz et al. (1971), further on some results derived therefrom (Wakker, 1991b, 1991c).

We prefer the algebraic approach to the topological approach not only because its axioms are more general, but also because we consider these axioms to be more natural. The primary empirical meaning of continuity with respect to a connected procluct topology is precisely the solvelbility condition that it implies. It is more natural to state this empirical meaning explicitly, than to reinforce it into a stronger condition, of which the further empirical implications for finite data sets are not clearly identified. Compare the beginning of Subsection 4.4.1.

A fundamental problem of continuity and solvability assumptions, and thereby of all preference axiomatizations in the literature, is that these assumptions are not merely technical. They add empirical implications to the other axioms, and it is not clear what precisely these empirical implications are. Proposition 30 and Example 23 illustrate the point: The example shows that a finite daten set can violate preference-tradeoff consistency without violating indifference-tradeoff consistency. As the proposition shows, the two 
versions of tradeoff consistency become equivalent under sol vability or continuity assumptions. These assumptions, therefore, do add empirical implications to indifference-tradeoff consistency, such as preference-tradeoff consistency. This phenomenon is not typical of the particular axioms studied here, but pertains to continuity and solvability axioms in general. Further discussion of this issue can be found in Krantz et al. (1971, Section 9.1) and Wakker (1988). The Archimedean axiom is not problematic in this respect because it does not add empirical implications (Adams, Fagot, \& Robinson, 1970, Theorem 3; Krantz et al. 1971, Section 6.5.1; Luce et al., 1990, Theorem 21.21; Pfanzagl 1968 Sections 6.6 and 9.5 ), or only infinitesimally so (Narens, 1985, Theorem 2.8.3), to the other axioms. The axiom merely reflects the mathematical convention of excluding nonstandard real numbers and does not affect interpretations of finite data sets. Separating out this empirically harmless technical axiom is another virtue of the algebraic approach.

\subsection{Appendix B: Preference-Tradeoff Consistency, and other Implications of Tradeoff Consistency}

Self-contained proofs of the theorems in the mein text could be obtained, adapting earlier proofs to indifference-tradeoff conditions. Wakker's (1989) Remark III.7.3, showing thet inany derivations of arditive conjoint measurement only need indifference versions of the additivity conditions plus monotonicity, would be used extensively, and several other modifications would be required. These proofs would take much space. Therefore, we have chosen an alternative approach. It will consist of deriving preference-tradeoff conditions from the indifference conditions used in the main text, and then invoking existing theorems. That is, we reverse the implications of Proposition 19. The derivation of this reversal is not elementary, and needs the technical assumptions. The reversal does not hold in general, e.g. for finite models, and, therefore, data violating preference-tradeoff consistency need not violate indifference-tradeoff consistency, as illustrated by the following example. This can abviously be a reason to check not only indifference versions of tradeoff consistency, but also preference versions, in data.

Example 23 Assume two states (healthy or ill) and four outcomes, $\alpha$ (airplane flight), $\beta$ (travel by boat), $\gamma$ (travel by Greyhound bus), $\lambda$ (long delay). Preferences are represented by $\left(f_{1}, f_{2}\right) \mapsto V_{1}\left(f_{1}\right)+V_{2}\left(f_{2}\right)$, with $V_{1}$ and $V_{2}$ defined in the following table. 


$\begin{array}{ccccc} & \alpha & \beta & \gamma & 6 \\ V_{1} & 1 & 0.9 & 0.3 & 0 \\ V_{2} & 0.99 & 0.44 & 0.33 & 0\end{array}$

With always $\lambda$ and $\gamma$ as gauge outcomes, $(\lambda, \alpha) \succcurlyeq(\gamma, \beta)$ and $(\lambda, \beta) \prec(\gamma, \gamma)$ imply $\alpha \beta \succcurlyeq *$ $\gamma^{\delta}$ whereas $(\alpha, \lambda) \preccurlyeq(\beta, \gamma)$ and $(\beta, \lambda) \succcurlyeq(\gamma, \gamma)$ imply $\beta \gamma \succ^{* *} \alpha \beta$. Preference-tradeoff consistency (Eq. 4.4) is violated, as well as its [sign-jcomonotonic versions. Indifferencetradeoff consistency holds vacuously because there are no non-reflexive indifferences. No nonzero $V_{1}$ difference for the first state can be matched exactly by a $V_{2}$ difference for the second state, the former always being multiples of 0.1 and the latter never.

In the rest of the proofs, we will oiten use wealk monotonicity conditions without explicit mention. We first sketch the main idea of the derivation of preference-tradeoff consistency from tradeoff consistency. Assume that we have a violation of preferencetradeoff consistency, i.e. four preferences $\alpha_{i} f \succcurlyeq \beta_{i} g, \gamma_{i} f \preccurlyeq \delta_{i} g, \alpha_{j} v \preccurlyeq \beta_{j} w_{2} \gamma_{j} v \succ \delta_{j} w$ hold as in $\mathrm{Eq}_{\text {. }}$ 4.4. Assume that we can worsen the $f_{k}$ for $k \neq i$ so much, and improve the $g_{k}$ for $k \neq i$ so much, that after these changes, $\alpha_{i} f \sim \beta_{i} g$ (Lemma 31). The preference $\gamma_{i} f \preccurlyeq b_{i} g$ will only be intensified by such changes. Imagine that, next, we carn improve outcome $\gamma$ and worsen outcome $\delta$ so much that after these changes, the indifference $\gamma_{i} f \sim \delta_{i} g$ results (Step 2 in the main proof of Proposition 30). The preference $\gamma_{j} v \succ \delta_{j} w$ will only be intensified by such changes. Imagine that, next, we can improve the $v_{k}$ for $k \neq j$ so much, and worsen the $w_{k}$ for $k \neq j$ so much, that after these changes, $\alpha_{j} v \sim \beta_{j} w$ (Step 3 of proof of Proposition 30). The preference $\gamma_{j} v \succ \delta_{j} w$ will only be intensified by such changes. After such manipulations, the special case of Eq. 4,4 has resulted where the first three preferences are indifferences. It can be demonstrated that such cases are excluded by tradeoff consistency (Corollary 28). The above manipulations extensively use technical conditions such as solvability. Changes as suggested may not always be possible, in which case alternative similar changes have to be devised, leading to a complex taxonomy of different cisises.

To begin our formal analysis, we first derive some preparatory results that may have interest on their own. Proposition 30 will then complete the proof. Henceforth, statements will often be combined by means of braces and square brackets such as in ["sign-) comonotonic) strong monotonicity, etc. If this notation is used then, for the signcomonotonic case, all texts, both those between square brackets and those between braces, are to be read. For the comonotonic case, the texts between braces are to be read but those 
between square brackets are to be skipped. For the case without sign- or oomonotonicity restrictions, all texts between square brackets and braces are to be skipped.

The following condition provides a useful tool in the subsexuent proofs. It can also serve as an alternative formulation of the tradeoff-consistency conditions defined in the main text, because it is equivalent under appropriate assumptions (Corollary 28) and is stated directly in terms of preferences. \{/Sign-/comonotonic\} strong indifference-tradeoff consistency holds if $\alpha_{i} f \sim \beta_{i} g, \gamma_{i} f \sim \delta_{i} g$, and $\alpha_{j} x \sim \beta_{j} y$ imply $\gamma_{j} x \sim \delta_{j} y$, whenever $\left\{\alpha_{i} f, \beta_{i} q_{,} \gamma_{i} f, \gamma_{i} g \in F\right.$ and $\alpha_{j} x_{i} \beta_{j} y, \gamma_{j} x, \delta_{j} y \in F^{\prime}$ for some [sign-]comoncones $F$ and $F^{*}$, where $i$ is nomull \{on $F$ \}.

Lemma 24. Assume weak ordering, weak monotonicity, and \{[sign-jcomonotonic\} stnong monotonicity. Then (/sign-/comonotonic) strong indiffenence-tradeoff consistency implies [sign-/comonotonic\} tradeoff consistency.

Proof: Suppose that the assumptions of the lemma hold, and that \{[sign-]comonotonic\} tradeoff consistency is violated. We derive a violation of [[sign-]comonotonic\} strong indifference-tradeoff consistency. There exist four indifferences $\alpha_{i} f \sim \beta_{i} g, \gamma_{i} f \sim \delta_{i} g$, $\alpha_{j} x \sim \beta_{j} y$, and $\gamma_{j} x \sim \delta_{j}^{k} y$, with $i$ and $j$ nonnull \{on appropriate [sign-]comonconess containing the relevant acts\} and $\delta^{\prime} \nsim \delta$, say, $\delta^{\prime} \prec \delta$. (Because of symmetries, other cases are similar.)

For $F=F^{\prime}=X^{n}$ (no sign- or comonotonic restrictions), we replace $\delta^{\prime}$ by $\delta$ in the last indifference, changing it into a strict preference by strong monotonicity, and, thus, generating a violation of strong indifference-tradeoff consistency. Under the nonexpected utility theories considered in this chapter, we have to ensure the appropriate sign- and comonotonicity conditions. We then proceed as follows. In each case hereafter, the changed acts will be in the same [sign-]comoncone as the original ones because the new outcomes are always between the original ones and $\beta$, thus satisfy all required sign- and comonotonicity conditions as do $\beta$ and the original outcome.

(a) If $\delta^{\prime} \prec \delta \leqslant \beta$, then we replace $\delta^{\prime}$ by $\delta$ in the llast indifference, turning it into a strict preference $\gamma_{j} x \prec \delta_{j} y$ by $\{[\mathrm{sigm}-]$ comonotonic $\}$ strong monotonicity. Thus, a violation of $\{[$ sign- $]$ comonotonic $\}$ strong indifference-tradeoff consistency results.

(b) If $\delta^{\prime} \prec \beta \prec \delta$, then we have $\gamma_{i} f \sim \delta_{i} g \succ \beta_{i} g \sim \alpha_{i} f$ implying $\gamma \succ \alpha$, but we also have $\gamma_{j} x \sim \delta_{j}^{y} y \prec \beta_{j} y \sim \alpha_{j} x$ implying $\alpha \succ \gamma$. A contradiction has resulted.

(c) If $\beta \approx \delta^{\prime} \prec \delta$, then we replace $\delta$ by $\delta^{\prime}$ in the second indifference, to obtain $\alpha_{i} f \sim \beta_{i} g$, 
$\gamma_{i} f>\delta_{i}^{*} g, \alpha_{j} x \sim \beta_{j} y$, and $\gamma_{j} x \sim \delta_{j}^{\prime} y . \quad\{$ Sign-Jcomonotonic $\}$ strong indifference-tradeoff consistency is violated by relabeling and symmetry.

In the remainder of this Appendix B, we will often use the following assumption.

Assumption 25 Weak ondering, weak monotonicity, and [sign-jcomonotonic\} trudeoff consistency hold.

Lemma 26 Under Assumption 25. (/sign-/comonatonic) strong monotonicity holds.

Proof: Let $F$ be a [sign-jcomoncone, or $X^{n}$, as the case may be. For $f, g \in F$, assume that $f_{j} \succcurlyeq g_{j}$ for all states $j$ and $f_{i} \succ g_{i}$ for some state $i$ that is nonmull on $F$. The following reasoning proves $f \succ g$. \{We assume, without loss of generality, that the acts in $F$ are rank-ordered from best to worst by the identity order.\}

Define $h=\left(f_{1}, \ldots, f_{i}, g_{i+1}, \ldots, g_{n}\right)$. Then $f \succcurlyeq h \succcurlyeq\left(g_{i}\right)_{i} h \succcurlyeq g$ \{with all acts contained in $F$ \}. It suffices to show $h=\left(f_{i}\right)_{i} h \succ\left(g_{i}\right)_{i} h_{\text {. }}$. Assume, for contradiction, that $\left(f_{i}\right)_{i} h \sim$ $\left(g_{i}\right)_{i} h$. Combining this indifference with $\left(f_{i}\right)_{i} h \sim\left(f_{i}\right)_{i} h$ implies $f_{i} g_{i} \sim_{F}^{*} f_{i} f_{i}$, writing $\left(f_{i}\right)_{i} h \sim\left(f_{i}\right)_{i} h$ twice gives $f_{i} f_{i} \sim_{F}^{*} f_{i} f_{i}$. \{[Sign-jcomonotonic $\}$ tradeoff consistency implies $f_{i} \sim g_{i}$, contradicting the assumed $f_{i}>g_{i}$

Lemma 27 Under Assumption 25 and solvability, \{/sign-jcomonotonic $\}$ strong indifference-tradeoff consistency holds.

Proof: We will only consider the, most complex, sign-comonotonic case. Assume, for contradiction, a violation of sign-comonotonic strong indifference-tradeoff consistency. We have $\alpha_{i} f \sim \beta_{i} g, \gamma_{i} f \sim \delta_{i} g$, and $\alpha_{j} x \sim \beta_{j} y$, and, say, $\gamma_{j} x \prec \delta_{j} y$, with all acts in appropriate sign-comoncones and with $i$ nounull on the relevant sign-comoncone. The strict preference implies that $j$ is nonnull on the relevant sign-comoncone because at lenst one of the latter two acts is not equivalent to $\alpha_{j} x \sim \beta_{j} y$.

First, assume $\alpha \succcurlyeq \gamma$. Then $\beta_{i} g \sim \alpha_{i} f \geqslant \gamma_{i} f \sim \delta_{i} g$. Transitivity and sign-comonotonic strong monotonicity (Lemma 26) imply $\beta \succcurlyeq \delta$. That is, $\alpha_{j} x \sim \beta_{j} y \succcurlyeq \delta_{j} y \succ \gamma_{j} x$. There exists, because of solvability, an $\varepsilon$ with $\varepsilon_{j} x \sim \delta_{j} y$ and, further, $\alpha \succ \varepsilon>\gamma$. (It can, but need not, be seen that, in general, $\varepsilon \succ \alpha$ is possible if state $j$ is nonnull on the set now considered, but is null elsewhere. In this case, we can always take $\varepsilon=\alpha$.) We have $\varepsilon_{j} x \in F^{r}$ because $\alpha_{j} x \in F^{\prime}$ and $\gamma_{j} x \in F^{\prime}$. Because $j$ is nonnull on $F^{\prime \prime}$, the equivalences 
$\alpha_{j} x \sim \beta_{j} y$ and $\varepsilon_{j} x \sim \delta_{j} y$ imply $\alpha \beta \sim_{s c}^{*} \varepsilon \delta$. Also $\alpha \beta \sim_{s c}^{*} \gamma \delta$ and $\gamma \nsim \varepsilon$, which violates sign-comonotonic tradeoff consistency.

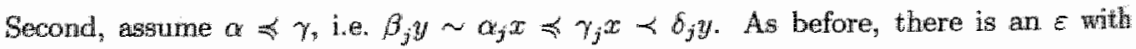
$\gamma_{j} x \sim \varepsilon_{j} y$ and $\beta \preccurlyeq \varepsilon \prec \delta$, so that $\varepsilon_{j} y \in F^{\prime}$. The equivalences $\alpha_{j} x \sim \beta_{j} y$ and $\gamma_{j} x \sim \varepsilon_{j} y$ imply $\alpha \beta \sim_{a c}^{*} \gamma \varepsilon$, but also $\alpha \beta \sim_{s c}^{*} \gamma \delta$ and $\delta \nsim \varepsilon$, which violates sign-comonotonic tradeoff consistency.

Both cases lead to a contradiction, therefore, $\gamma_{j} x \sim \delta_{j} y$ must hold.

Corollary 28 Under weak ordlering, weak monotonicity, and \{(sign-jcomonotonic\} strong monotonicity, \{/sign-lcomonotonic\} atrong indifference-tradeoff consistency implies \{/signjcomonotonic] tradeoff consistency. Under weak ordering, weak monotonicity, and solvability, \{/sign-/comonotonic\} tradeoff consistency implies \{/sign-/comonotonic\} strong indifference-tradeolf consistency.

The following corollary demonstrates that tradeoff consistency implies a restriction of the sure-thing principle for indifferences, corresponding to the theory under consideration.

Corollary 29 Under Assumption 25 and solvability, $\left(\alpha_{i i} f \sim \alpha_{i} g\right) \Leftrightarrow\left(\beta_{i} f \sim \beta_{i} g\right)$ \{whenever the acts are [sign-/comonotonic\}.

Proof: [Let the mentioned acts be contained in a [sign-]comonotonic set $F$.\} If $i$ is null \{on $F\}$, then $\alpha_{i} f \sim \beta_{i} f$ and $\alpha_{i g} \sim \beta_{i} g$, from which everything follows. Next suppose that $i$ is not null \{on $F\}$. Then, by Lemma $27, \alpha_{i} f \sim \alpha_{i} f, \beta_{i} f \sim \beta_{i} f$, and $\alpha_{i} f \sim \alpha_{i} g$, imply $\beta_{i} f \sim \beta_{i}, g$.

Proposition 30 Under Assumption 25 and solvability, \{/sign-jcomonotonic $\}$ prefenencetradeoff consistency holds.

Proof: We derive one final preparatory lemma within this proof. Ends of proofs within proofs are indicated by $Q E D$, not. by $\square$.

Lemma 31 Assume weak ondering, weak monotonicity, and soivability. Let $F$ be either $X^{x^{\prime}}$, or a comoncone, or a stign-comoncone, Let $\alpha_{i} f \nLeftarrow \beta_{i} g$ and $\gamma_{i} f \preccurlyeq(\prec) \delta_{i} g$ where all four acts nore contained in $F$. Then thene exist $\bar{f}, \bar{g}$ with $\alpha_{i} \bar{f}, \beta_{i} \bar{g}, \gamma_{\hat{n}} \bar{f}, \delta_{i} \vec{g} \in F$, such that either $\left(\alpha_{i} \bar{f} \sim \beta_{i} \bar{g}\right.$ and $\left.\gamma_{i} \bar{f} \preccurlyeq(\prec) \delta_{i} \bar{g}\right)$ or $\left(\alpha_{i} \bar{f} \succ \beta_{i} \bar{g}_{i} \gamma_{i} \bar{f} \preccurlyeq(<) \delta_{i} \bar{g}\right.$, and $\bar{f}_{j} \leqslant \bar{g}_{j}$ for all $j \neq i)$. Th the latter case, $\alpha \succ \beta$. Funthermore, in any case, we can have $\vec{f}_{j} \leqslant f_{j}$ and $\bar{g}_{j} \succcurlyeq g_{j}$ for all $j \neq i$. 
Proof: The idea underlying the proof is to consider, one by one, those pairs of outcomes with $f_{j} \succ g_{j}$ for $j \neq i$, and to move either $f_{j}$ down or $g_{j}$ up in preference until either indifference $\alpha_{i} f \sim \beta_{i} g$ results, or $f_{j}$ and $g_{j}$ become indifferent and still $\alpha_{i} f \succ \beta_{i} g$. In the latter case, we continue with the next pair $f_{j} \succ g_{j}$ if existing. To maintain the appropriate sign- and comonotonicity requirements, we move the $f_{j}$ s down for $j$ rank-ordered after i, starting with the last-rank-ordened $j$, and the $g_{j}$ s up for $j$ rank-ordered before $i$, starting with the first-rank-ordered $j$. The details are as follows.

Let $\alpha_{i} f, \beta_{i} g, \gamma_{i} f, \delta_{i} g \in F$, such that $\alpha_{i} f \succcurlyeq \beta_{i} g$ and $\gamma_{i} f \preccurlyeq(<) \delta_{i} g$. As before, we will only consider the most complex case, where $F$ is a sign-comoncone. Without loss of generality it can be assumed that the acts in $F$ are rank-ordered from best to worst by the identity ordering. Further, we may assume that $f=\alpha_{i} f$ and $g=\beta_{i} g$.

STEP 1. We will "push the $g_{j} \prec f_{j}$ up towards $f_{j}$ " and next "push $f_{j} \succ g_{j}$ down toward $g_{j}$," until either an indifference $\alpha_{i} f \sim \beta_{i} g$ results, or $f_{j} \preccurlyeq g_{j}$ for all $j \neq i$ and still $\alpha_{i} f \succ \beta_{i} g$ (then $\alpha$ must be much better than $\beta$ ). If one of these cases already holds, then the rest of Step 1 can be skipped and the proof continues with Step 2. Assume $\alpha_{i} f \succ \beta_{i} g$ and $f_{j} \succ g_{j}$ for at least one $j \neq i$.

STEP 1.1 [ Pushing the $g_{j} \prec f_{j}$ up towards $f_{j}$ ]. Assume that there is a $j<i$ with $f_{j} \succ g_{j}$ (otherwise, go to Step 1.2). Let $l$ be the minimal (hence, rank-ordered first) index for which $f_{l} \succ g l$. Consider the act $\left(f_{l}\right), g$. This act is an element of $F$, because $g_{t-1} \succcurlyeq f_{l-1} \succcurlyeq f_{l} \succ g_{l} \succcurlyeq g_{l+1}$. If $\left(f_{l}\right)_{l} g \leqslant f$ replace the old act $g$ by $g=\left(f_{i}\right)_{l} g$ and start again with Step 1. Otherwise, i.e. if $(f)_{l} g \succ f \succ g$, then there exists, because of solvability, an $\varepsilon$ with $\varepsilon_{l} g \sim f$. We have $f_{l} \succ \varepsilon \succ g_{l}$ so that $\varepsilon_{l g} \in F^{\prime}$. Replace the original act $g$ by $g=\varepsilon_{l} g$ and go to Step 2 .

STEP 1.2 [Pushing $f_{j}>g_{j}$ down towards $\left.g_{j}\right]$. Assume that there is no $j<i$ with $f_{j} \succ g_{j}$. Then there is a $j>i$ with $f_{j} \succ g_{j}$. Let $l$ be the maximel (hence, rank-ordered last) index for which $f_{t} \succ g_{t}$. Consider the act $\left(g_{l}\right)_{l} f$. This act is an element of $F$, because $f_{i-1} \succcurlyeq f_{i} \succ g_{i} \succcurlyeq g_{t+1} \succcurlyeq f_{i+1}$. If $\left(g_{i}\right)_{l} f \succcurlyeq g$, replace the old act $f$ by $f=\left(g_{l}\right)_{i} f$ and start again with Step 1. Otherwise, i.e. if $f \succ g \succ(g) f$, then there exists, because of solvability, an $\varepsilon$ with $\varepsilon_{l} f \sim g$. We have $f_{l} \succ \varepsilon \succ g_{l}$ and $\varepsilon_{l} f \in F$. Replace the original act $f$ by $f=\varepsilon_{u} f$ and go to Step 2 . 
STEP 2. Because there are only finitely many states, the procedure in Step 1 ends after finitely many repetitions. Define $\bar{f}$ and $\bar{g}$ as the newly constructed acts $f$ and $g$ resulting from Step 1. We have either $\alpha_{i} \bar{f} \sim \beta_{i} \bar{g}$ or $\alpha_{i} \bar{f} \succ \beta_{i} \bar{g}$ and $\bar{f}_{j} \preccurlyeq \bar{g}_{j}$ for all $j \neq i$. In what follows, $f$ and $g$ refer again to the original acts $f$ and $g$. For the act $\bar{f}$ we have $\bar{f}_{l}=f_{t}$ if $l<i$ and $\bar{f}_{l} \preccurlyeq f_{l}$ otherwise. Then $\gamma_{i} \bar{f} \preccurlyeq \gamma_{i} f$. For the act $\bar{g}$ we have $\bar{g}_{l}=g$ if $l>i$ and $\bar{g}_{l} \succcurlyeq g_{i}$ otherwise. Then $\delta_{i} \bar{g} \succcurlyeq \delta_{i} g$. Because the $f_{j}$ s for $j<i$ have remained unaffected, and the $f_{j}$ s for $j>i$ have been moved down in preference, still $f_{i-1} \succcurlyeq \gamma \succcurlyeq f_{i+1}$ and $\gamma_{i} \bar{f} \in F$. Similarly, $\delta_{i} \bar{g} \in F$. We have $\gamma_{i} \bar{f} \preccurlyeq \gamma_{i} f \preccurlyeq(\prec) \delta_{i} g \preccurlyeq \delta_{i} \bar{g}$ and, hence, $\gamma_{i} \bar{f} \preccurlyeq\left(\langle) \delta_{i} \bar{g}\right.$. This completes the proof of Lemma 31 . QED

In the rest of the proof of Proposition 30 , we, again, consider only the most complex case, the sign-comonotonic case. Let $y$ satisfy sign-comonotonic tradeoff consistency. Let $F, F^{\prime}$ be two sign-comoncones. Assume that there are acts

$$
\begin{aligned}
& c_{i} f \geqslant \beta_{i} g, \quad \alpha_{j} x \leqslant \beta_{j} y_{n} \\
& \gamma_{i} f \leqslant \delta_{i} g, \quad \gamma_{j} x \succ \delta_{j} y_{,}
\end{aligned}
$$

such that $\alpha_{i} f, \beta_{i} g, \gamma_{i} f, \delta_{i} g \in F, i$ is nomnull on $F$, and $\alpha_{j} x, \beta_{j} y, \gamma_{j} x, b_{j} y \in F^{\prime}$ (the strict preference implies that $y$ is nonnull on $F^{\prime}$ ).

STEP 1. In this step we show that either $\alpha \preccurlyeq \gamma$ and $\beta \preccurlyeq \delta$, or $\alpha \succcurlyeq \gamma$ and $\beta \succcurlyeq \delta$. Assume $\alpha \prec \gamma$. Sign-comonotonic strong monotonicity (Lemma 26) implies $\delta_{i} g \succcurlyeq \gamma_{i} f \succ \alpha_{i j} f \succ \beta_{i} g$. We have $\beta \prec \delta$. Next assume $\alpha \succcurlyeq \gamma$. Then $\beta_{j} y \succcurlyeq \alpha_{j} x \succcurlyeq \gamma_{j} x \succ \delta_{j} y$ and $\beta \succ \delta$.

STEP 2. In this step, we show that there exist $\bar{\alpha}_{i} f \sim \bar{\beta}_{i} g, \bar{\gamma}_{i} f \sim \bar{\delta}_{i} g, \bar{\alpha}_{j} x \prec \bar{\beta}_{j} y$, and $\bar{\gamma}_{j} x \succ \bar{\delta}_{j} y$ with $\bar{\alpha}_{i} f, \bar{\beta}_{i} g_{3}, \bar{\gamma}_{i} f, \bar{\delta}_{i g} \in F$, and $\bar{\alpha}_{j} x, \bar{\beta}_{j} y, \bar{\gamma}_{j} x, \bar{\delta}_{j} y \in F^{v}$. In each of the following two cases, $\bar{\alpha}$ and $\bar{\gamma}$, or $\bar{\beta}$ and $\bar{\delta}$, are between $\alpha$ and $\gamma$, or $\beta$ and $\delta$, respectively, in preference, and, therefore, will satisfy all the required sign- and comonotonicity restrictions.

First, assume $\alpha \preccurlyeq \gamma$ and $\beta \leqslant \delta$. See Figure 4.1.A. We have $\delta_{i} g \succcurlyeq \gamma_{i} f \succcurlyeq \alpha_{i} f \succcurlyeq \beta_{i} g$. Solvability implies that there is a $\bar{\beta}$ with $\alpha_{i} f \sim \bar{\beta}_{i} g$, and a $\bar{\delta}$ with $\gamma_{i} f \sim \bar{\delta}_{i} g$. We can get

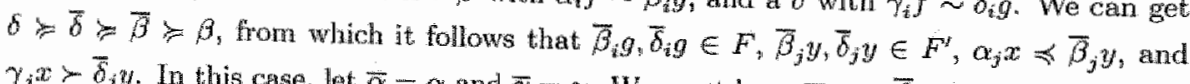
$\gamma_{j} x \succ \bar{\delta}_{j y}$. In this case, let $\bar{\alpha}=\alpha$ and $\bar{\gamma}=\gamma$. We must have $\bar{\alpha}_{j} x<\bar{\beta}_{j} y$ because the other case, $\bar{\alpha}_{j} x \sim \bar{\beta}_{j} y$, would imply, by Lemma 27 , that $\bar{\gamma}_{j} x \sim \bar{\delta}_{j} y$, a contradiction.

Second, assume $\alpha \ni \gamma$ and $\beta \succcurlyeq \delta$. See Figure 4.1.B. Similar as in the first case, solvalbility implies that thare is an $\bar{\alpha}$ with $\alpha \succcurlyeq \bar{\alpha} \succcurlyeq \gamma$ and $\bar{\alpha}_{i} f \sim \beta_{i} g$, and a $\bar{\gamma}$ with $\bar{\alpha} \succcurlyeq \bar{\gamma} \geqslant \gamma$ and $\bar{\gamma}_{i} f \sim \delta_{i} g$. Furthermore, $\bar{\alpha}_{i} f, \bar{\gamma}_{i} f \in F, \bar{\alpha}_{j} x, \bar{\gamma}_{j} x \in F^{\prime}, \bar{\alpha}_{j} x \preccurlyeq \beta_{j} y$, and 


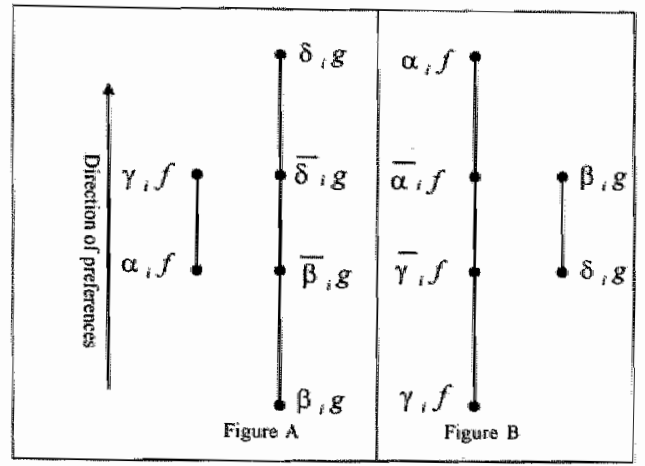

Figure 4.1:

$\bar{\gamma}_{j} x \succ \delta_{j} y$. In this case let $\bar{\beta}=\beta$ and $\bar{\delta}=\delta$. Lemma 27 implies that we must have $\bar{\alpha}_{j} x \prec \bar{\beta}_{j} y$.

As a preparation for the following step, we rename, for notational convenience, $a=$ $\bar{\alpha}, \beta=\bar{\beta}, \gamma=\bar{\gamma}$ and $\delta=\bar{\delta}$ so that we have $\alpha_{i} f \sim \beta_{i} g, \gamma_{i} f \sim \delta_{i} g, \alpha_{j} x \prec \beta_{j} y$, and $\gamma_{j} x \succ \delta_{j} y$. Furthermore, as before, either $\alpha \preccurlyeq \gamma$ and $\beta \preccurlyeq \delta$, or $\alpha \succcurlyeq \gamma$ and $\beta \succcurlyeq \delta$. Because of symmetry, we may essume hereafter $\alpha \preccurlyeq \gamma$ and $\beta \leqslant \delta$.

STEP 3. We will rule out all cases except $\gamma \succ \delta \succcurlyeq \beta \succ \alpha$. By Lemma 31, we can find $\widetilde{x_{i}}, \widetilde{y}$ ("pushing the $x_{j}$ s up and the $y_{j} \mathrm{~s}$ down") such that either $\alpha_{j} \widetilde{x} \sim \beta_{j} \tilde{y}$ and $\gamma_{j} \widetilde{x} \succ \delta_{j} \widetilde{y}$, or $\alpha_{j} \widetilde{x} \prec \beta_{j} \widetilde{y}$ and $\gamma_{j} \widetilde{x} \succ \delta_{j} \widetilde{y}$ with $\widetilde{x}_{l} \succcurlyeq \widetilde{y_{l}}$ for all $l \neq j$. Furthermore, $\alpha_{j} \widetilde{x}, \beta_{j} \widetilde{y}_{1} \gamma_{j} \widetilde{x}, \delta_{j} \widetilde{y} \in F^{\prime}$. The first case leads to a contradiction because of Lemma 27 , and we, therefore, assume that the second case holds. This implies, in particular, $\alpha \prec \beta$, because $\widetilde{x}_{i} \succcurlyeq \widetilde{y}$ for all $l \neq j$.

Again by Lemma 31 , we can find $\bar{x}, \bar{y}$ ("pushing" the $x_{j}$ s down and the $y_{j}$ s up) such that either $\gamma_{j} \bar{x} \sim \delta_{j} \bar{y}$ and $\alpha_{j} \bar{x} \prec \beta_{j} \bar{y}$, or $\gamma_{j} \bar{x} \succ \delta_{j} \bar{y}$ and $\alpha_{j} \bar{x}<\beta_{j} \bar{y}$ with $\bar{x}_{i} \preccurlyeq \bar{y}_{l}$ for all $l \neq j$. Furthermore, $\alpha_{j} \overline{x_{1}}, \beta_{j} \bar{y}, \gamma_{j} \bar{x}, \delta_{j} \bar{y} \in F^{\prime \prime}$. The first case leads to a contradiction because of Lemma 27 , we, therefore, assume that the second case holds. This implies, in particular, $\gamma>\delta_{3}$ becenuse $\bar{x}_{l} \leqslant \overline{y_{l}}$ for all $l \neq j$.

STEF 4. We have $\gamma \succ \delta \succcurlyeq \beta \succ \alpha$. This will lead to the final contradiction. Signcomonotonic strong monotonicity (Lemma 26) implies $\beta_{i} f \succ \alpha_{i} f \sim \beta_{i} g$ and $\delta_{i} f \prec \gamma_{i} f \sim$ 


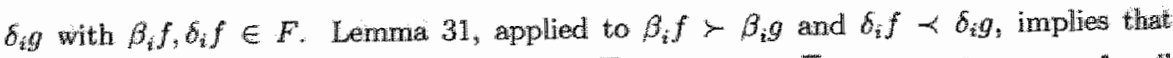
we can find $\bar{f}, \bar{g}$ such that $\beta_{i} \bar{f} \sim \beta_{i} \bar{g}$ and $\delta_{i} \bar{f}<\delta_{i} \bar{g}$ (with $\bar{f}_{j}<f_{j}$ and $\bar{g}_{j} \succcurlyeq g_{j}$ for all $f \neq i$ ). The second case in that lemma, implying the impossible $\beta-\beta$, camnot occur bere Furthermore, $\beta_{i} \bar{f}, \beta_{i}, \delta_{i}, \bar{f}, \bar{g} \in F$. A contradiction with Corollary 29 has resulted. The proof is complete.

\subsection{Appendix C: Further Proofs}

This appendix completes the proofs. We display one more preparatory lemma.

Lemrina 32 Assume weak ondering, \{fsign-/comonotonic $\}$ weak and strong monotonicity. and solvatility. If a \{/sign-fcomanotonic\} standand sequence $\alpha^{0}, \alpha^{1}, \ldots$ is increasing, then $\alpha^{k+11}>\alpha^{k}$ for all $k$. If it is decreasing, then $\alpha^{k+1}<\alpha^{k}$ for all $k$.

Proof: Obvious.

Proof of Theorems 10 and 13, and Observations 11 and 14: For the implications (i) $\Rightarrow$ (ii) in both theorems, necessity of the Archimedean and the tradeoff-consistency axioms follows from Eq. 4.3 as explained in the main text, and the other conditions are obvious. We, therefore, assume the Statements (ii) and derive the corresponding Statements (i) and the uniqueness results in the Observations. Statement (ii) and Proposition 30 imply the preference versions of the tracleoff-consistency axioms.

We first consider the SEU model of Theorem 10, and the uniqueness results of Observation 11. These follow from Theorem 5 in Wakker (1991c) for two or more nonnull staties. For exactly one nomull state, the existence of a representing function on the outcomes follows from Theorem 2.2 of Krantz et al. (1971), and then everything follows. For the trivial case of a trivial preference relation, all results are trivial.

We next turn to the CEU model of Theorem 13 and Observation 14. These follow from Theorem 8 in Wakker (1991c) for the nondegenerate case. If one comoncone has only null states, then all outcomes are equivalent, the corresponding constant acts being contained in this one comoncone. Then the preference relation is trivial, and so are the results for this case. The only remaining case is the degrenerate case of exactly one nonmull state in every comoncone. Then, as before, Theorem 2.2 of Krantz et al. (1971) implies the existence of a representing function $U$ on the outcomes. For every act $f$ in a comoncone with nonnull 
Btate $i, O\left(f_{i}\right)$ determines the preference value of act $f$. From this observation, everytling follows.

We next turn to the claims at the end of Subsection 4.4.1. The following lemma prepares for Observation 34 .

Lemma 33 Assume weak ondering, at least two nonnull states, weak monotonicity, and solvability. Then, for all outcomes $\alpha, \beta$, there exist acts $f, g$ and a nonnull state $j$ such that $\alpha_{j} f \sim \beta_{j} g$. This implies $\alpha \beta \sim * \alpha \beta$ and, hence, reflexivity of $\sim$ ".

Proof: Assume that states 1 and 2 are nonnull. Let $\alpha, \beta$ be outcomes, and $h$ an arbitrary fixed act. W.l.o.g. assume $\alpha \succcurlyeq \beta$ and $\beta_{1} \alpha_{2} h \succcurlyeq \alpha_{1} \beta_{2} h$. We may add " $\succcurlyeq \beta_{1} \beta_{2} h$ " By solvability, there exists a $\delta$ with $\alpha_{1} \beta_{2} h \sim \beta_{1} \delta_{2} h$. Writing the indifference twice and applying Eq. 4.2 with $j=1$ implies $\alpha \beta \sim^{*} \alpha \beta$.

The following observation, formalizing the claim at the end of Subsection 4.4.1, was stated for real-walued outcomes, and without formal proof, by Kobblerling \& Wakker (2002a, see Chapter 3).

Observation 34 In Statement (ii) of Theorem 10, tradeoff consistency (d) can be replaced by the requirement that strong monotonicity holds and that $\sim$ " is transitive. If there are at least two nonnull states, transitivity of $\sim^{*}$ can be replaced by the requarement that $\sim^{*}$ is an equivalence relation.

Proof: $I_{t}$ is obvious that SEU implies strong monotonicity. If $\succcurlyeq$ is trivial, then $\sim^{*}$ is empty and everything follows. In the degenerate case of exactly one nonnull state, $\alpha \beta \sim^{*} \gamma \delta$ if and only if $\alpha \sim \beta$ and $\gamma \sim \delta$ for all outcomes $\alpha, \beta, \gamma, \delta$, and everything follows. Note that in the trivial and the degenerate cases, $\sim^{*}$ is transitive and symmetric but not reflexive and, hence, is not an equivalence relation. The rest of this proof assumes two or more nonirull states.

To demonstrate that SEU implies transitivity of $\sim^{*}$, ensume $\alpha \beta \sim \sim^{\prime \prime} \gamma \delta$ and $\gamma \delta \sim \sim^{*}$ or. The latter $\sim$ implies that $\gamma_{j} f \sim \delta_{j} g$ and $\sigma_{j} f \sim \tau_{j} g$ for some $f, g$, and nonnull $j$. $\alpha \beta \sim^{*} \gamma \delta$ implies $U(\alpha)-U(\beta)=U(\gamma)-U(\delta)$, by Eq. 4.3. This equality, $\gamma_{j} f \sim \delta_{j} g$, and substitution of expected utility imply $\alpha_{j} f \sim \beta_{j} g$. This indifference and $\sigma_{j} f \sim \gamma_{j} g$ imply $\alpha \beta \sim \sim^{*} \sigma \tau$. Transitivity of $\sim$ * follows. Symmetry of $\sim^{*}$ was demonstrated in Observation 
9. Reflexiveness is implied by Lerma 33. Note that the latter requires solvability. We conclude that $\approx *$ is an equivalence relation.

Conversely, assume that Statement (ii) in Theorem 10 holds but with (d) replaced by strong monotonicity and transitivity of $\sim$. We derive tradeof consistency. Assume $\alpha \beta \sim^{\prime \prime} \gamma \delta$ and $\alpha^{\prime} \beta \sim^{*} \gamma \delta, \sim^{*}$ is symmetric (Observation 9) and transitive, i.e. $\alpha \beta \sim^{*} \alpha \beta$. Therefore, there exist acts $f$ and $g$ and a nonnull state $i$ such that $\alpha_{i} f \sim \beta_{i} g$ and $\alpha_{i}^{\prime} f \sim \beta_{i} g$, and, hence, $\alpha_{i} f \sim \alpha_{i}^{\prime} f$. By strong monotonicity, $\alpha \sim \alpha^{\prime}$ follows. Tradeoff consistency holds.

Because Lemmas 20 and 21 are used in the proof of Corollary 15, we give their proofs prior to that of Corollary 15.

Proof of Lemma 20: This result was proved in Wakker (1989, Lemma III.3.3). It can also be inferred from Krantz et al. (1971, Section 6.2.13), where it is stated for three or more dimensions.)

The following lemma prepares for the proof of Lemma 21.

Lemma 35 Assume continuous weak ordering with respect to a connected product topology on $X^{n}$. The restriction of $\succcurlyeq$ to $X$ (through identification with constant acts) is continuous.

Proof: Consider a set $\{\alpha \in X: a \succ \beta\}$, and an element $\gamma$ thereof. The set $T=\{x \in$ $\left.X^{n}: x \succ(\beta, \ldots, \beta)\right\}$ is open in $X^{n}$. For $(\gamma, \ldots, \gamma)$ (as for all other elements of $T$ ), there exists a product set $A_{1} \times \cdots \times A_{n} \subset T$ of open sets $A_{j} \subset X$ that contains $(\gamma, \ldots, \gamma)$. The intersection $A_{1} \cap \cdots \cap A_{n}$ is a neighborhood of $\gamma$ in $\{\alpha \in X: \alpha \succ \beta\}$, proving that the latter set is open. Similarly, $\{\alpha \in X: \alpha \prec \beta\}$ is open.

Proof of Lemma 21: In the degenerate case of exactly one nonnull state in Lemma. 21, continuous weak ordering with respect to separable connected product topology implies that $\succcurlyeq$ on $X$ is continuous (Lemma 35), then that there exists a function representing it (well-known, e.g. Wakker, 1989, Theorem III.3.6), and, finally, that there exists a countable order-dense subset of $X$ (Krantz et al., 1971, Theorem 2.2). Therefore, Lemma 21 is done if the following lemma is proved.

Lemma 36 Assume continuous weak ordering with respect to a connected product topology, Under weak and \{comonotonic\} strong monotonicity, every bounded (comonotonic) standand sequence is finite. 
Proof: (This proof can also be inferred from Krantz et al, 1971, Section 6.12.3, for the noncomonotonic case, and from Wakker, 1991b, Lemma 4.2, for the comonotonic case.) Assume that $\left(\alpha^{k+1}\right)_{i} g_{j} f \sim\left(\alpha^{k}\right)_{i} G_{j} f$ for all $k$, \{with all acts contained in a comoncone $F$ and with $i$ and $j$ nonnull \{on $F$ \}. Assume that the standard sequence is increasing, i.e. $G \succ g$. (The other case is similar.) By Lemma 32, $\alpha^{k} \succ \alpha^{k-1}$ for all $k$. It is abvious that $\alpha^{\circ}$ can serve as an outcome less preferred than all elements of the standard sequence. Let $\sigma$ be an ontcome more preferred than all $\alpha^{k}$ and assume, for contradiction, that the standard sequence is infinite. The set $\left\{\beta: \beta<\alpha^{k}\right.$ for some $\left.k\right\}$ is, by Lemme 35, a union of open sets and, hence, is open. It contains $\alpha^{\text {ll }}$ and is nonempty. Its complement is $\left\{\beta: \beta \succcurlyeq \alpha^{k}\right.$ for all $\left.k\right\}$, and is nonempty because it contains $\sigma$. For contradiction with connectedness, we prove that it is open.

\{Comonotonic restrictions for $F$ may require that all $\alpha^{k}$ are less preferred than some fixed outcome $\sigma^{\prime}$, for $\left(\alpha^{k}\right)_{i} g_{j} f$ and $\left(\alpha^{k-1}\right)_{i} G_{j} f$ to be contained in $F$. For example, if the rank-ordering for $F$ is the identity, if $i=n$, and $j<n-1$, then $\sigma^{\prime}=f_{n-1}$. We then first consider $\sigma \preccurlyeq \sigma^{\prime}$. In this case, for any outcome $\beta$ with $\alpha^{0} \leqslant \beta \leqslant \sigma$, it follows that $\beta_{i} g_{j} f$ and $\beta_{i} G_{j} f$ are contained in $F$. $\}$ Consider the preferences $\sigma_{i} G_{j} f \succ \sigma_{i} g_{j} f \geqslant\left(\alpha^{k+1}\right)_{i} g_{j} f \succ$ $\left(\alpha^{k}\right)_{i} g_{j} f \sim\left(\alpha^{k-1}\right)_{i} G_{j} f$, where the strict preferences follow from \{comonotonic\} strong: monotonicity. From $\sigma_{i} G_{j} f \succ \sigma_{i g_{j}} f \succ\left(\alpha^{k-1}\right)_{i} G_{j} f$ and solvability (Lemma 20) we conclude that $\gamma_{i} C_{j} f \sim \sigma_{i} g_{j} f$ for some $\gamma$, by weak monotonicity $\sigma \succ \gamma \succ \alpha^{k m l}$. This holds for all $k$.

Similarly, for any $\tau$ instead of $\sigma$ such that $\tau \succ \alpha^{k-1}$ for all $k$, there exists a $\gamma^{\prime}$ such that $\tau \succ \gamma^{\prime} \succ \alpha^{k-1}$ for all $k$. $\left\{\gamma^{\prime}=\sigma^{\prime}\right.$ if $\tau \succ \sigma^{\prime}, \gamma^{\prime}$ is constructed as $\gamma$ above if $\tau \leqslant$ $\sigma^{\prime}$. $\}$ By Lemma 35 the set $\left\{\delta \in X: \delta \succ \gamma^{\prime}\right\}$ is open, by transitivity it is contained in $\left\{\beta: \beta \succcurlyeq \alpha^{k}\right.$ for all $\left.k\right\}$ and it contains $\tau$, in short, it is a neighborhood of $T$ within $\left\{\beta: \beta \succcurlyeq \alpha^{k}\right.$ for all $\left.k\right\}$. The latter set is open. A contradiction has resulted, and the standard sequence must be finite. $Q E D$

Proof of Corollary 15: The implication (i) $\Rightarrow$ (ii) is elementary. Next assume (ii). The conditions of Theorems 10 and 13 have been established in the main text and in Lemmas 20,26, and 21. Theorems 10 and 13 can be used to imply the Statements (i), except for continuity of the utility functions. Continuity of utility is proved as in Wakker (1988, Theorem 3.1) and Wakker (1993a, Lemma A.1). The extra continuity requirement in the uniqueness result is obviously satisfied. An alternative proof of this Corollary can be obtained from Proposition 30 and existing results in the literature for the topological approach, i.e. Wakker (1989, Theorems IV.2.7 and VI.5.1 and Remark A3.1). 
Proof of Obserwation 16: For a sign-comonotonic set, we can take one fixed n-thale of decision weights that are all nonnegative. For the states of nature with gain-outconnes they are derived from $W^{4}$, for the states of nature with loss-outcomes they are derwed from the dual of $W^{-}$. If at least one of these weights is positive, then we normalize by dividung each weight by the total sum of clecision weights, leading to probabilities and an SEU-model. If all decision weights are zero, then the preference relation is trivial on the particular set, and we take the probabilities arbitrary and utillity constant. The latter case can occur for general CPT (if $W^{+}(A)=0=W^{-}(S-A)$ for an event $A$ ), but will be excluded in our main results by an assumption that $z$ is "truly mixed."

Proof of Theorem 17 and Obserwation 18: These results follow from Proposition 30 and Theorem 6.3 in Wakker \& Twersky (1993).

Proof of Proposition 19: The preference-tradeoff consistency conditions imply not only weak monotonicity, but also the corresponding strong monotonicities (Wakker 1989 Lemma VI.4.10; Wakker \& Tversky 1993, Lemma A2, 2). They also imply the corresponding strong equivalence-tradeoff conditions, because indiflerences $\alpha_{i} f \sim \beta_{i} g, \gamma_{i} f \sim \delta_{i} g$, and $\alpha_{j} x \sim$ $\beta_{j} y_{1}$ imply both the triple $\alpha_{i} f \preccurlyeq \beta_{i} g_{i} \gamma_{i} f \succcurlyeq b_{i} g_{\text {, and }} \alpha_{j} x \geqslant \beta_{j} y$ and the triple $\alpha_{i} f \geqslant \beta_{i j}$, $\gamma_{i} f \leqslant \delta_{i} g$, and $\alpha_{j} x \leqslant \beta_{j} y$, therefore both $\gamma_{j} x=\delta_{j} y$ and $\gamma_{j} x \leqslant \delta_{j} y$. We conclude that the corresponding strong equivalence-tradeof consistency holds. The proposition follows from Lemma 24 . 


\section{Chapter 5}

\section{An Index of Loss Aversion}

To a considerable extent, risk aversion as it is commonly observed is caused by loss aversion. This chapter proposes a quantitative index of loss aversion. Under prospect theory, the proposal leads to a decomposition of risk attitude into three distinct components: intrinsic utility, probability weighting, and lose aversion. The main theorem shows how the index of loss aversion of different decision makers can be compared through observed choices. Our analysis cests doubt upon utility functions with constant relative risk aversion, commonly assumed in the finance and macroeconomics literature. These functions cannot be reconciled with the empirical findings on loss aversion. Utility with constant absolute risk aversion can be. ${ }^{1}$

\subsection{Introduction}

Given the abundant evidence of the empirical failures of expected utility, the development of tractable alternative theories for decision under risk is desirable (Rabin \& Thaler, 2001; The Economist, 2001; Starmer, 2000). A popular alternative is Quiggin's (1981) rank-dependent utility. Tversky \& Kahneman's (1992) new version of prospect theory generalizes rank-dependent utility by adding loss aversion as a new component of risk aversion. Thus, the new prospect theory combines the mathematical elegance of Quiggin's theory with the empirical realism of Kahneman \& Tversky's (1979) original prospect theory.

\footnotetext{
The xesults in this chapter were first fromulded in (Kobberling \& Wakks, 2002c).
} 
Loss aversion reflects the observed behavior that agents are more sensitive to losses than to gains, resulting in a utility function that is steeper for losses than for gains. The phenomenon is empirically well established, and has played a central role in recent explanations of the equity premium puzzle (Benartzi \& Thaler, 1995). However, little theory has been developed so far, and no axiomatization exists as yet for loss aversion. Quantifications and parametrizations have been chosen on an ad hoc basis. This chapter is the first to propose a theoretically founded index of loss aversion to govern the exchange rate between gain and loss utility units. This proposal leads to a clear decomposition of risk attitude into three distinct components: basic utility, probability weighting, and loss aversion. We hope that this simple decomposition will contribute to the tractability of prospect theory for applications in economics.

Our analysis will shed new light on the appropriateness of parametric utility families: In particular, utilities with constant relative risk tolerance, commonly used in finance and macrocconomics, cannot be reconciled with empirical findings on loss aversion. Constant absolute risk tolerance performs better in this regard. Other members of the HARA family (Merton, 1971), or new parametric families yet to be developed, deserve more attention.

Although we will formulate our results for decision under risk, they can be applied to welfare theory as well, where loss aversion reflects the special sensitivity of consumers to decreases in income. Sign-dependence of welfare transfers, assigning special importance to transfers that reduce the number of individuals facing decreases in income, provides a promising way to generalize welfare evaluation models (Chateauneuf, Gajdos, \& Wilthien, 2002).

Definitions are given in Section 5.2 , and Section 5.3 presents the proposed index of loss aversion. Section 5.4 gives a comparative preference foundation by means of Yaari's (1969) acceptance sets. Section 5.5 presents empirical findings, and discusses the role of loss aversion in Rabin's (2000) paradox. Section 5.6 gives conceptual arguments for our proposal, and compares it to other proposals that were put forward in the literature. Section 5.7 concludes. The analysis of our index for parametric utility families is somewhat complex and, therefore, is given in Appendix A of this chapter. Appendix B provides
proofs. 


\subsection{Prospect Theory}

Outcomes are monetary, with $I R$ the outcome set. We assume that the agent perceives one outeome as the reference potnt, and the other outcomes as changes with respect to this meference point. The reference point often is the status quo. By rescaling the outcomes, we may assume that 0 is the reference point. Then, gains are positive amounts and losses are negative amounts. A lottery, denoted by $P=\left(p_{1}, x_{1} ; \ldots ; p_{n}, x_{n}\right)$, assigns a nonnegative probability $p_{i}$ to each outcome $x_{i}$, where $\sum_{i=1}^{n} p_{i}=1$. Throughout, the outcomes in $P$ are ordered from best to worst, with $x_{1} \geq \ldots \geq x_{k} \geq 0>x_{k+1} \geq \ldots \geq x_{n}$ for some $0 \leq k \leq n$. $L$ denotes the set of all lotteries. Each outcome $x$ is identified with the riskless lottery $(1, x)$. The preferences of an agent over lotteries are denoted by $\succcurlyeq$, with indifference denoted by $\sim$.

We will next define the new version of prospect theory, introduced by Tversky \& Kahneman (1992). The theory splits up a lottery into its gain part, where all negative outcomes are replaced by 0 , and its loss part, where all positive outcomes are replaced by 0 . It them applies different rank-dependent formulas (Quiggin, 1981) to the gain and loss part, and, finally, adds these two formulas. The theory, thus, generalizes rank-dependent utility by bringing in the loss aversion of original prospect theory (Kahneman \& Tversky, 1979), and varies upon original prospect theory by transforming probabilities in Quiggin's (1981) rank-dependent manner. Consequently, it generalizes rank-dependent utility by allowing for different risk attitudes for gains than for losses, an empirically desirable generalization. Formally, prospect theory holds if an evaluation function CPT represents preferences (i.e, $P \succcurlyeq Q \Leftrightarrow C P T(P) \geq C P T(Q)$ for all lotteries $P, Q)$, where CPT is defined as follows.

(i) There exists a utility function $U: \mathbb{R} \rightarrow \mathbb{R}$, which is continuous and strictly increasing with $U(0)=0$.

(ii) There exist weighting functions $u^{+}, w^{-\prime}:[0,1] \rightarrow[0,1]$, which are continuous and strictly increasing with $w^{+}(0)=w^{-}(0)=0$ and $w^{+}(1)=w^{-(1)}=1$.

(iii) $\operatorname{CPT}\left(p_{1}, x_{1} ; \ldots ; p_{n}, x_{n}\right)=\sum_{i=1}^{n i} \pi_{i} U\left(x_{i}\right)$, with $\pi_{i}=w^{+}\left(p_{1}+\cdots+p_{i}\right)-w^{+}\left(p_{1}+\cdots+p_{i-1}\right)$ for $i \leq k$ (rank-dependent utility for gains with respect to $\left.w^{+}\right)$and $\pi_{i}=w^{-}\left(p_{i}+\cdots+p_{n}\right)-w^{-}\left(p_{i+1}+\cdots+p_{n}\right)$ for $i>k$ (rank-dependent utility for losses with respect to the dual of $\left.w^{-}\right)^{2}$

\footnotetext{
${ }^{2}$ In particular, $\pi_{1}=a^{+}\left(p_{1}\right)$ if $k \geq 1$ and $\pi_{n}=w^{-}\left(p_{n}\right)$ if $k<\pi$
} 


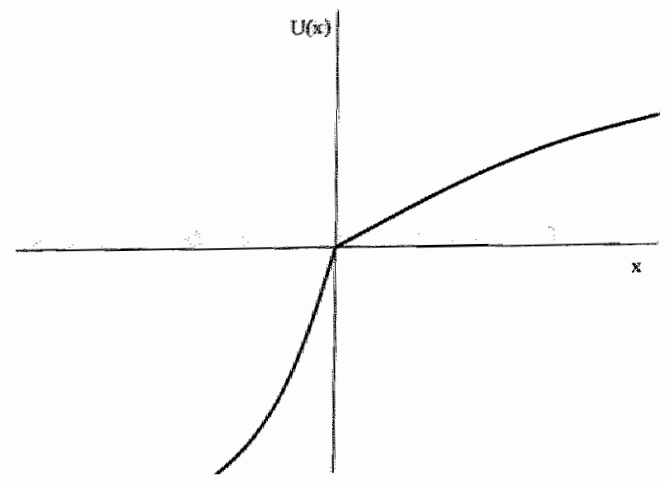

Figure 5.1: A typical $U$

\subsection{An Index of Loss Aversion}

We assume that there exists a basic utility function $u$ reflecting the intrinsic value of outcomes for the agent. Because of the perception of a reference point, however, subjects process losses differently than gains. The observable utility $U$ is a composition of a loss aversion index $\lambda>0$, cepturing the different processing of gains and losses, and the basic. whility z. Formally,

$$
U(x)=\left\{\begin{array}{cc}
u(x) & \text { if } x \geq 0 \\
\lambda u(x) & \text { if } x<0
\end{array}\right.
$$

Typically, people pay more attention to losses, $\lambda$ exceeds 1 , and $U$ is steeper for losses than for gains.

Without specifying a scaling convention for the gain-loss exchange rate of $u$, it is impossible to separate $u$ and $\lambda$ through observed choices. Twersky \& Kahneman (1992), who used the above decomposition, assumed that $u(x)=x^{\alpha}$ for gains and $u(x)=-|x|^{\beta}$ for losses. This amounts to the implicit scaling convention that $u(1)=-u(-1)=1$, implying $\lambda=\frac{-U(-1)}{U(1)}$ (see Section 5.6). This scaling convention requires specification of the unit of payment. We propose another scaling convention that is independent of the unit of payment. 
A typical $U$ is depicted in Figure 5.1. The utility function $U$ has a kink at zero and is smooth everywhere else. It is plausible that the kink is caused by lass aversion, and not by the intrinsic value of outcomes. That is, it is plausible that the basic utility function 2. is smooth (differentiable) at zero. We, therefore, define $\lambda=\frac{b_{t}^{t}(0)}{U_{l}^{t}(0)}$ as the loss aversion inder $U_{f}^{\prime}(0)$ denotes the left and $U_{f}^{\prime}(0)$ the right derivative of $U$ at zero. We assume that $U_{i}(0)$ and $U_{1}^{\prime}(0)$ exist and are positive and finite. The assumption of smoothness of $u$ at zero serves, so to say, as our scaling convention for the gain-loss exchange rate.

The ratio $\frac{U_{f}^{\prime}(0)}{U^{\prime}(0)}$ was suggested informally as a measure of loss aversion by Benartzi \& Thaler (1995, p. 74 1.5-6). Our chapter formalizes their suggestion. The index $\lambda$ is invariant under changes of scale of $U$ and is, therefore, well defined. It is, moreover, invariant under scale transformations of the outcomes, i.e. it is independent of the unit of payment. Further discussions and justifications of our proposal are given in the following sections, and summarized in the conclusion.

\subsection{Comparative Loss Aversion}

Comparisons of loss aversion can be naturally formulated through Yaari's (1969) acceptance sets, leading to a preference foundation for comparative loss aversion. Our preference condition will imply that weighting functions and atility functions are the same for different agents, which entails a considerable restriction. Similar restrictions, however, also applied to the first results on utility and; subsequently, probability weighting, as new components of risk attitude. The classical results on utility did not consider loss aversion and probability weighting and, thus, implicitly assumed that these are the same for all agents (Arrow, 1953, 1965; Bernoulli, 1738; Rothschild \& Stiglit, 1970; Pratt, 1964; Yaari, 1969 with same subjective probabilities for different agents). The first results on probability weighting either assumed linear utility (Yaari, 1987; Schmeidler 1989 w.r.t. probabilistic mixtures), or did not disentangle probability weighting and utility curvature (Chateanneuf \& Cohen, 1994; Chateauneuf \& Tallon, 2002; Chew, Karni, \& Safra, 1987). All the references just mentioned did not consider loss aversion, which then obviously is the seme for all agerts also. Schmidt \& Zank (2002) will characterize strong riglk aversion through th joint condition on utility curvature, probability weighting, and our index of loss aversion without, however, disentangling these components. Their result renders support to our index as a natural component of risk attitude. 
Assume two agerves, 1 and 2, whose preferences over $L$, denoted by $\succcurlyeq_{1}$ and $\geqslant 2$ can be morleled through prospect theory wh utility functions $U_{1}, U_{2}$, underlying basic witity functions $u_{1}, u_{2}$, loss suersion indexes $\lambda_{1}, \lambda_{2}$, and weighting functions $w_{1}^{+}, w_{1}^{-}, w_{2}^{+}, w_{2}^{-}$, respectively. We denote the reference point of each agent by 0 , where this can refer to different absolute levels of wealth for the two agents.

$L^{t}$ denotes the set of lotteries with no loss outcomes and $L^{-}$the set of lotteries with no gain outcomes. A lottery is mixed if it is neither contained in $L^{+}$nor in $L^{-}$, so that it yields both gains and losses with positive probability. For an outcome $x$, we deffie $A_{1}(x)=\left\{P^{\prime} \in L \mid P \& x\right\}$ to be agent 1 's acceptance set, i.e. the set of lotteries that the agent prefers to receiving the sure outcome $x$. The gain acceptance set $A_{1}^{*}(x)=A_{1}(x) \cap L^{*}$ restricts attention to lotteries with no loss ontcomes. Similarly, the lass acceptance set is $A_{1}^{-}(x)=A_{1}(x) \cap L^{-}$. Agent $2: s$ acceptance sets $A_{2}(x), A_{2}^{+}(x)$, and $A_{2}^{-}(x)$ are defined in the same way.

Assume that agent 2's acceptance sets are contained in those of agent 1 . This meansi that agent 2 is more risk averse than agent 1 in Yaari's sense. As will be discrussed ir. Section 5.6, empirical studies suggest that loss aversion is a major factor in risk aversion. It can, therefore, be expected that difierences in observed risk attitudes of different agents are, to a considerable extent, caused by differences in loss aversion, which is observed in mixed lotteries only. This chapter characterizes the extreme but not implausible case where no significant differences are found fot gain-or loss-lotteries, but differences do occur for mixed gambles (Barberis, Huang, \& Santos 2001, p. 17). That is, the gain and loss acceptance sets of the two agents are the same, but for mixed gambles the acceptance sets. of agent 1 are larger than those of agent 2. The following theorem shows that this special case of Yaari's preference condition is characterized in terms of the loss aversion index introduced in Section 5.3, while implying the same $u$ and $w$.

Theorem 37 Assume that the preferences of agents 1 and 2 can be modeled through prospect theory, such that $U_{11}^{\prime}(0), U_{11}^{\prime}(0), U_{42}^{\prime}(0)$, and $U_{12}^{\prime}(0)$ are positive ard finite. Then the following Statements (i) and (ii) are equivalent.

(i) The following three conditions hold:

(a) $w_{2}^{+}=w_{1}^{+}$and $w_{2}^{-\infty}=w_{1}^{+}$;

(b) $u_{1}=\sigma u_{2}$ for some $\sigma>0$ (a.e. $u_{1}$ and $u_{2}$ ane strategrically equzalent):

(c) $\lambda_{2} \geq \lambda_{1}$ 
(ii) The following two conditions hold:

(a) $A_{2}^{-1}(x)=A_{1}^{+}(x)$ and $A_{2}^{-}(-x)=A_{1}^{-}(-x)$ for all $x \in \mathbb{R}^{+}$;

(b) $A_{2}(x) \subset A_{1}(x)$ for all $x \in \mathbb{R}$.

In Theonem $37(\mathrm{i})$, the conditions $(b)$ and (c) are equivalent to the equality $\sigma U_{2}(x)=\left\{\begin{array}{ll}U_{1}(x) & x \geq 0 \\ \gamma U_{1}(x) & x \leq 0\end{array}\right.$ for some $\sigma>0, \gamma \geq 1$

The characterizing preference condition can, equally well, be reformulated in terms of certainty equivalents instead of acceptance sets. That is, Statement (ii) in the theorem can be replaced by

- For all $P$ in $L^{+}$and in $L^{-}$, the certainty equivalents of the two agents coincide;

- For all $P$ in $L$, the certainty equivalent of agent 2 does not exceed that of agent 1 .

Under rank-dependent utility and prospect theory, the implications of Yaari's famous definition of conparative risk aversion by means of acceptance sets are as yet unknown. Under expected utility, Yaari's condition characterizes concave transformations of utility, The restriction of Yaari's condition in Theorem 37 also characterizes concave transformations of utility but of a special kind; with a kink at zero and linear otherwise. We have, therefore, characterized a more restrictive preference condition in a more general model. The restrictive condition precisely captures an important new empirical phenomenon, i.e. loss aversion.

A way to disentangle all components of risk attitude remains a topic for future research. It would require isolating loss aversion without imposing identical acceptance sets over the pure gain and the pure loss domain.

\subsection{Empirical Findings and Suggestions}

Recent empirical studies suggest that loss aversion is a major factor in observed risk aversion (Kahneman, Knetsch, \& Thaler, 1991). Benartzi \& Thaler (1995) explained the equity premium puzzle by myopic loss aversion. Investors are not willing to accept a high variety of outcomes, which might cause short-rum losses, even if the high variety generales gains in the long run. Many other empirical studies have confirmed the importance of 
loss aversion (Barberis, Huang, Santos, 2001; Bateman et al., 1997; Cachon \& Camerer, 1996; Growzy \& Potters, 1997; Hershey. Kunreuther \& Schoemaker, 1982; Hershey \& Schoemaker, 1985; Pryne, Laughhunn \& Crum, 1980, 1981; Schmidt \& Traub, 2000: Thaler et al., 1997; Twersky \& Kahneman, 1991). It is remarkable that no theoretical foundation exist as yet for such an irmortant empinical concept.

Rabin (2000) and Rabin \& Thaler (2001) provided further evidence for the dependence of decisions on reference points. They assumed an agent who would always turr down a $50-50$ gamble of losing $\$ 10$ or gaining $\$ 11$, independently of the initial wealth, and derived an anomaly. Under expected utility, this person would have to reject any bet with a $50 \%$ risk of losing \$100, no matter how high the potential gajns would be. Such an extreme degree of rish avergion is not realistic. Rabin and "Thaler suggested that loss aversion, with initial wealth as reference point, provides a better explanation for the observed preferences. than concave utility.

The obtained separation of the three components is crucial if variations in the reference point are consiclered. Shallev (2000) considered such variations in game theory, and Bleichrodt, Pinto, \& Wakker (2001) used Shalev's model in utility elicitation. A crucial assumption in these models is that basic utility is the same for different reference points. We can then observe the loss aversion index $\lambda$ by comparing the kink of utility $U$ at at point when it is the reference point with the kink of $U$ at that same point when another point is the reference point. A scaling convention need not be imvoked anymore.

There have been many debates on the difference between ordinal and cardinal utility (Arrow, 1965; Ng, 1997; Samuelson, 1938). For advocates of a candinal concept of utlity prior to risk, the difference between such a concept and risk attitude can be ascribed to probability weighting and loss aversion. To what extent loss aversion, comprised in $U$, should be part of a riskless cardinal concept of utility sinch as used in welfare evaluations, is open to debate, both from the nomative and from the empirical perspective.

We expect that the common assuraption of linear utility on small domains will be approximately verified on $[0, \epsilon]$ and $[-\epsilon, 0]$ for $\epsilon>0$ small. The marginal utility on the latter interval will, however, continue to exced the marginal utility on the former to a significant degree. The interval [-e, $\epsilon]$ will continue to exhibit considerable deviations from linearity, also for small 6 . This finding reflects loss aversion, i.e s $\lambda$ exceding one. Therefore, lotiteries $(.5, M+\epsilon ; .5, M-\epsilon)$ will exhibit considerable risk aversion, also for small $c>0$, if $M$ is zero but this will happen less so if $M$ is not zero. Empirical support for 
the latter claim can be found in Hershey, Kunreuther \& Schoemaker (1982) and Hershey \& Schoemaker (1985). Rabin and Thaler alluded to this phenomenon when they suggested lioss aversion as an explanation for the paradoxical phenomena described in their papers.

\subsection{Alternative Definitions of Loss Aversion}

Alternative scaling conventions for the gain-loss exchange rate of $u$ can be devised, leading to different definitions of loss aversion. For example, a $\tau>0$ can be chosen and $-u(-\tau)=$ $w(\tau)(=U(\tau))$ can be set, leading to $\lambda=\frac{-U(-\tau)}{U(\tau)}$. Such alternative conventions usually imply that $u$ is nondifferentiable at zero. Tversky \& Kahneman's (1992) approach is a special case hereof, with $T=1$. The same convention of $\tau=1$ was used by Fishburn (1977) and Holthausen (1981) within the expected utility framework, by Luce (1991) and Luce \& Fishburn (1991, Eq, 4) in their preference foundation of CPT, and by Bleichroth \& Miyanoto (2002). Our definition $\lambda=\frac{U_{f}^{\prime}(0)}{\partial_{t}^{\prime}(0)}$ can be considered the limiting case where $\tau$ approaches zero. Because our definition does not depend on the unit of payment, it is the same across different countries, and does not require readjustment after inflation or a change of currency such as recently in Europe. Decompositions of an overall utility function into an underlying basic utility and additional psychological factors were considered before by Bleichrodt, Pinto, \& Wakker (2001), Cowen (1992, the additional factor lieing equity), Karni \& Safra (2002, the additional factor being faimess), Loomes \& Sugden (1986, the additional factor being disappointment), and Shalev (2000).

The loss aversion index $\lambda$ is of a psychological nature. It is affected by strategically irrelevant perceptions of the reference point, such as underlying the known discrepancies between willingness to pay and willingness to accept (Bateman et al., 1997). In some situations it may be deemed desirable to let the basic utility $u$ have a kink at zero, if losses bring genuine extra inconveniences not matched by corresponding gains. Whether such a change of marginal utility around zero is drastic but still smooth, or abrupt, is not ant empirical question because we do not observe stakes smaller than pennies. It is a question of pragmatic modeling. For consumers who typically receive a fixed anount of money each month and spend it continuously, we think that an abrupt change of marginal utility precisely at the current reference point is implausible (Arrow, 1951, p. 432). Tf there are, however, genuine empirical or pragmatic reasons for an intrinsic kink of utility at some point, and if this point happens to be the reference point, then we think that this kink 
should be incorporated in the basic utility function and not in loss aversion.

In an interesting study, Huber, Ariely, \& Fischer (2001) specified the true preference system of a principal, and asked participants ("agents") to represent the principals in decisions. Remarkably, there still was considerable loss aversion, in deviation from the true preferences. The authors write: "In many settings, one cannot tell whether loss aversion is a bias or merely a reflection of the fact that losses have more emotional impact than gains of equal magnitude. In our choice and rating tasks, however, we found clear evidence that agents motivated to accurately represent the preferences of others gave more weight to negative outcomes than is appropriate" (p. 88).

The scaling convention that we chose to define loss aversion seens to be plausible, but does not cover all concepts of loss aversion advanced in the literature. As these concepts have varied from one context to the other, there can, unfortunately, not be one definition that optimally meets all objectives. Examples can be devised in which our definition is counter to some terminologies used before. For example, assume that $U$ is differentiable at zero so that $\lambda$ is one, $U$ is the identity for positive outcomes, and $U$ is very concave for negative outcomes. Then $-U(-y)$ exceeds $U(y)$ for all $y>0$, a condition which is sometimes interpreted as loss aversion (Kahneman \& Tversky, 1979; Markowitz, 1952). In our terminology, the loss aversion index is one, suggesting no loss aversion. Instead, we say that $U$ is more concave for losses than for gains. Such discrepancies between our definition and others in the literature are, however, not empirically plausible.

Besides the definition of loss aversion of Kahneman \& Tversky (1979) and Markowitz (1952), just mentioned, other definitions of loss aversion have been proposed. Wakker \& Tversky (1993) defined loss aversion as the requirement that $U^{\prime}(-x) \geq U^{\prime}(x)$ for all positive $x$ and provided a preference foundation. An alternative foundation was given by Neilson (2002, Theorem 3). The definition was extended and tested empirically by Schmidt (2002) and Schmidt \& Traub (2000), and characterized by Bleichrodt \& Miyamoto (2002) for the health domain. Neilson (2002, Theorem 2) also considered and characterized a weaker condition, requiring that $U(x) / x \leq U(y) / y$ for all $x<0<y$. Bowman, Minehart, \& Rabin (1999) and Breiter et al. (2001) required $U^{\prime \prime}(-x) \geq U^{\prime}(y)$ for all positive $x, y$. These definitions all have in common that loss awersion cannot be separated from utility curwature. Benartzi \& Thaler (1995) related loss aversion to the local behavior of utility near zero, as this chapter does. In our approach, loss aversion is a third component of risk attitude, separated from probability weighting and basic ntility, thereby from the curvature 
of utility for gains and losses. We feel that the kink at zero is more naturally modeled as a separate conceptual component than as part of (basic) utility. The convenience of the resulting three-component decomposition of risk attitude motivated our study of the factor $\lambda$. Whether $\lambda$ is called an index of loss aversion or otherwise is, in the end, only a terminological issue.

\subsection{Concluding Remarks}

So far, loss aversion has been a well-established empirical phenomenon, but preference foundations and theoretical studies have been virtually absent in the literature. This chapter has presented an index of loss aversion and has analyzed some empirical applications. Our proposal is based on the assumption that differentiability of basic utility at the reference point is plausible. The resulting loss aversion index is independent of the unit of payment. A comparative preference foundation of the index has been given. We have compared our proposal to other, informal, proposals in the literature. The main motivation for our proposal was that it leads to a clear decomposition of risk attitude into three distinct components.

\subsection{Appendix A: Implications for Parametric Fami- lies of Utility}

The cases of $U_{\uparrow}^{\prime}(x)$ and $U_{j}^{\prime}(x)$ equal to zero or infinity have been excluded in our definition of loss aversion. In several empirical studies, however, (e.g. Tversky \& Kahneman, 1992), constant relative risk tolerance has been assumed, with utility functions of the form

$$
U(x)=\left\{\begin{array}{cc}
x^{r} & \text { if } x \geq 0 \\
-s(-x)^{t} & \text { if } x<0
\end{array}\right.
$$

A drawback of these functions is that they have extreme, zero or infinite, deriwatives at zero whenever the power is not one. This drawback complicates the definition of loss aversion. ${ }^{3}$

${ }^{3}$ This draw back was called to our attention by A C.D. Donkers, personal communication. 
Twersky \& Kahneman (1992) found $r=t=0.88$. In such an exceptional case with $r=t$,

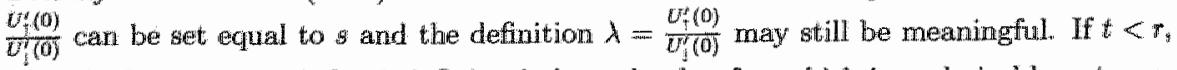
then the loss aversion index is infinite, independently of $s$, which is undesirably extreme. In thiss case no basic utility function, differentiable at zero, can be defined. Further, for all $s$ we then have $U(x)>-U(-x)$ for sufficiently large $x>0$, contrary to empirical findings.

The most commonly found case of power utility is $r<t$. More precisely, utility is concave for gains, convex for losses (Abdellaoui, 2000; Heath, Huddart, \& Lang, 1999), and closer to linear for losses than for gains $(0<r<t<1$; Fenmema \& Van Assen, 1998). Then the loss aversion index is zero, independently of $s$ and, again, no basic utility function can be defined. In this case another complication arises, demonstrating once more the problematic nature of constant relative risk tolerance. No matter how large $s$ is, $U(x)$ exceeds $-U(-x)$ for all $x$ in a sufficiently small neighborhood of zero, contrary to the common empirical findings. In summary, constant relative risk tolerance always entails: the existence of $U(x)>-U(-x)$ contrary to loss aversion, unless the exceptional case of $r=t$.

We next demonstrate that constant absolute risk tolerance, i.e. exponential utility, can accommodate the desirable features of loss aversion described in the preceding paragraphs. Take

$$
x(x)= \begin{cases}\frac{1-e^{-\mu x x}}{\mu} & \text { for all } x \geq 0 \\ \frac{e^{\mu x x}-1}{\nu} & \text { for all } x<0\end{cases}
$$

as basic utility, and define:

$$
U(x)=\left\{\begin{array}{cc}
\frac{1-e^{-\mu x}}{\mu z} & \text { for all } x \geq 0 \\
\lambda\left(\frac{e^{2 x}-1}{w}\right) & \text { for all } x<0 .
\end{array}\right.
$$

The division by $\mu$ and $\nu$ is a normalization guaranteeing that the left and right derivatives of the basic utility are one at zero. For $\mu$ or $\nu$ equal to zero, the functions are to be taken linear. In this family, $\mu$ controls the concavity of utility for gains, $\nu$ the convexity of utility for losses, and $\lambda$ controls loss aversion. For $\lambda \geq 1$ and $0<\nu<\mu$, this family exhibits some desirable features regarding loss aversion discussed above. We have $-U(-x)>U(x)$ for all $x>0$ and, even stronger, $U^{\prime}(-x)=\lambda e^{-\mu x}>e^{-\mu x}=U^{\prime}(x)$ for all $x>0$. The latter 
inequality is a strong form of loss aversion that has been considered in the literature. Pratt's (1964) measure of concavity $A(x)=-U^{\prime \prime}(x) / U^{\prime}(x)$ satisfies $0<-A(-x)=y<$ $\mu=A(x)$ for all $x>0$, showing that $U$ is convex for losses, concave for gains, and closer to linear for losses than for gains, again in agreement with the empirical findings.

\subsection{Appendix B: Proofs}

Proof of Theorem 37: $(i) \rightarrow(i i)$ : Let $(i . a-c)$ hold. It is to be shown that (ii.a-b) hold, of which only (ii.b) is elaborated, because (ii.a) are trivial. Let $x \in \mathbb{R}$ and $P \in A_{2}(x)$, i.e. $C P T_{2}(P) \geq U_{2}(x)$. Let $P=\left(p_{1}, x_{1} ; \ldots ; p_{n}, x_{n}\right)$ with $x_{1} \geq \ldots \geq x_{k} \geq 0>x_{k+1} \geq$ $\ldots \geq x_{n}$ for some $0 \leq k \leq n$. Define $P^{+}=\left(p_{1}, x_{1} ; \ldots ; p_{k}, x_{k} ; 1-p_{1}-\cdots-p_{k, 1}, 0\right)$ and $P^{-}=\left(1-p_{k+1}-\cdots-p_{n}, 0 ; p_{k+1}, x_{k+1} ; \ldots ; p_{n}, x_{n}\right)$. Then $C P T_{2}(P)=C P T_{2}\left(P^{*}\right)+$ $C P T_{2}\left(P^{-}\right)=C P T_{1}\left(P^{+}\right)+\frac{\lambda_{y}}{\lambda_{1}} C P T_{1}\left(P^{-}\right) \leq C P T_{1}(P)$. If $x \geq 0$ then $U_{3}(x)=U_{1}(x)$, and, therefore, $U_{\mathrm{n}}(x) \leq C P T_{1}(P)$. If $x \leq 0$ then $U_{1}(x)=\frac{\lambda_{1}}{\lambda_{4}} U_{2}(x) \leq \frac{\lambda_{1}}{\lambda_{2}} C P T_{2}(P) \leq$ $\frac{\lambda_{1}}{\lambda_{2}} C P T_{1}\left(P^{+}\right)+C P T_{1}\left(P^{-}\right) \leq C P T_{1}(P)$. Hence, $P \in A_{1}(x)$ and, therefore, $A_{2}(x) \subset A_{1}(x)$.

(ii) $\rightarrow(i)$ : Let $(i i . a-b)$ hold. It is to be shown that $(i . a-c)$ hold. $U_{2}$ and $U_{1}$ are continuous and strictly increasing and $\succcurlyeq_{2}$ and $\succcurlyeq_{1}$ coincide on $L^{+}$and $L^{-}$. Standard uniqueness results (Wakker, 1994, Theorem 12) imply that $w_{2}^{+}(p)=w_{1}^{+}(p)$ and $w_{2}^{\prime}(p)=$ $w_{1}^{-}(p)$ for all $p \in[0,1]$. Furthermore, $U_{2}(x)=\alpha U_{1}(x)+\beta$ for all $x \in \mathbb{R}^{*}$ and $U_{2}(x)=$ $\gamma U_{1}(x)+\delta$ for all $x \in \mathbb{R}^{-}$with $\alpha, \beta, \gamma, \delta \in \mathbb{R}$ and $\alpha, \gamma>0$. Because $U_{2}(0)=U_{1}(0)=0$ and $U_{2}(1)=U_{1}(1)=1$ it follows that

$$
U_{2}(x)=\left\{\begin{array}{cc}
U_{1}(x) & x \geq 0 \\
\gamma U_{1}(x) & x \leq 0
\end{array}\right.
$$

It is left to show that $\gamma \geq 1$. Let $P=(p, 1 ; 1-p,-1)$ such that $C P T_{2}(P)<0$. Because $U_{2}$ is continuous, there exists a $y \in \mathbb{I R}$ with $U_{2}(y)=C P T_{2}(P)$, implying $P \in A_{2}(y)$. We have, with the inequalities inplied by $(i i . b), w_{1}^{+}(p) U_{1}(1)+w_{1}^{-}(1-p) \gamma U_{1}(-1)=w_{2}^{+}(p) U_{2}(1)+$ $w_{2}^{-}(1-p) U_{2}(-1)=C P T_{2}(P)=U_{2}(y)=\gamma U_{1}(y) \leq \gamma C P T_{1}(P)=\gamma w_{1}^{+}(p) U_{1}(1)+\gamma w_{1}^{-}(1 \ldots$ p) $U_{1}(-1)$. This implies $\gamma \geq 1$. 


\section{Chapter 6}

\section{Comparative Loss Aversion in}

\section{Prospect Theory}

This chapter points out the difficulties in quantifying loss aversion for general situations with nonmonetary outcomes. A theorem is given that slzows how relative comparisons of loss aversion can, nevertheless, be made through observed choices. It generalizes the main theorem in (Köbberling \& Wakker, 2002c, see Chapter 5)."

\subsection{Introduction}

Loss aversion is an important empirical phenomenon. It reflects the observed behavior that aggents are more sensitive to losses than to gains. Its empirical relevance was discussed in (Kobberling \& Wakker, 2002c, see Chapter 5). Prospect theory (Kahnemen \& Tversky, 1979; Tversky \& Kahneman, 1992) is a decision model that can incorporate loss aversion. Kobberling \& Wakker (2002c, see Chapter 5) proposed a theoretically founded index of loss awersion to gowern the exchange rate between gain and loss utility units for the case that outcomes are monetary. By rueans of this index interpersonal comparisons of loss anversion can be made.

For the proposed loss awersion index it was crucial that the outcomes can be identified with an interval on the real line. This assumption is natural in a monetary background, but in many other applications it is not: For instance, if outcomes correspond to health states,

\footnotetext{
Tha author thanks Peter P. Whiker for helpful connents and suggestions.
} 
the set of outcomes ig usually discrete and finite; this is also the case in choice settings whth a finite number of alternative choices, as jt appears in many individual choice situations or in social choice theory. In situations with general outcomes there is no obvious choice for an index of loss aversion as an absolute quantitative concept. Nevertheless, also for general outcomes it is possible to perform relative comparisons of loss aversion.

Section 6.2 introduces prospect theory for general outcomes, and discusses difficulties in defining an index of loss aversion. Section 6.3 demonstrates how the loss aversion of two agents can be cormpared through observed choices without referring to an index of loss. avension, and provides a generalization of the main theorem in (Kobberling \& Wakker, $2002 c$, see Chapter 5). Section 6.4 concludes. Proofs are collected in the Appendix of this chapter.

\subsection{Prospect Theory for General Outcomes}

The set of outcomes is denoted by $X$. A lottery, denoted by $P=\left(p_{1}, x_{1} ; \ldots ; p_{n}, x_{i n}\right)$, assigns a nonnegative probability $p_{i}$ to each outcome $x_{i}$, where $\sum_{i=1}^{n} p_{i}=1$. Each outcome $x$ is identified with the riskless lottery $(1, x)$. The set of all lotteries is $L(X)$. The preferences of an agent over lotiteries are denoted by $\succcurlyeq$, with indifference denoted by $\sim$, and strict preference denoted by $\succ$. A designated outcome $r \in X$ serves as the reference point for the agent, with respect to which all other outcomes are either gains, if $x \succ r$, or losses, if $x \prec r$. The outcomes in $P$ are ordered from best to worst, such that $x_{1} \succcurlyeq \ldots \geqslant x_{k} \succ r>x_{k+1} \succcurlyeq \ldots \succ x_{n}$ for some $0 \leq k \leq n$.

Next, the new version of prospect theory, introduced by Tversky \& Kahneman (1992), is defined. Prospect theory holds if an evaluation function CPT represents preferences (i.e., $P \succcurlyeq Q \Leftrightarrow C P T(P) \geq C P T(Q)$ for all lotteries $P, Q)$, where CPT is defined as follows.

(i) There exists at atity function $U: X \rightarrow \mathbb{R}$ with $U(r)=0$.

(ii) There exist weighting functions $w^{\text {th }}, w^{-}:[0,1] \rightarrow[0,1]$, which are continuous and strictly increasing with $w^{+}(0)=w^{-(}(0)=0$ and $w^{+}(1)=w^{-}(1)=1$.

(iii) $\operatorname{CPT}\left(p_{1}, x_{1} ; \ldots ; p_{n,}, x_{n i}\right)=\sum_{i=1}^{n} \pi_{i} U\left(x_{i}\right)$, with

$$
\begin{aligned}
& \pi_{i}=w^{+}\left(p_{1}+\cdots+p_{i}\right)-w^{+1}\left(p_{1}+\cdots+p_{i-1}\right) \text { for } \leq k \text { and } \\
& \pi_{i}=u^{-}\left(p_{i}+\cdots+p_{n}\right)-w^{-}\left(p_{i+1}+\cdots+p_{n}\right) \text { for }>k^{2}
\end{aligned}
$$

\footnotetext{
${ }^{2}$ In particular, $\pi_{1}=w^{+}\left(p_{1}\right)$ if $k \geq 1$ and $\pi_{n}=w^{-}\left(p_{n}\right)$ if $k<n$.
} 
The perception of a reference point causes agents to process losses differently than gains. Usually, more attention is paid to potential losses than to potential gains, so that the observed negative utility assigned to losses is bigger than the utility assigned to comparable gains. Köbberling \& Wakker (2002c, see Chapter 5) discussed the idea of a basic utility function $u$ reflecting the intrinsic value of outcomes for the agent. The observable utility $U$ is a composition of a loss aversion index $\lambda>0$, and the basic utility u. Formally,

$$
U(x)=\left\{\begin{array}{cl}
u(x) & \text { if } x \succcurlyeq r \\
\lambda u(x) & \text { if } x \prec r
\end{array}\right.
$$

Typically, people pay more attention to losses, i.e. $\lambda$ exceeds 1 . Nevertheless, without specifying a scaling convention it is impossible to separate $u$ and $\lambda$ through observed choices. For monetary outcomes and reference point zero, Tversky \& Kahneman (1992) suggested the scaling convention $u(1)=1=-u(-1)$ and $\lambda=-U(-1)$. Kabberling \& Wakker (2002c, see Chapter 5 ) proposed an altemative scaling convention: The loss aversion index $\lambda$ was chosen as the quotient of the left and the right derivative at zero of the observable utility function $U$. For both conventions it is crucial thet the outcomes correspond to an interval on the real line. In more general situations with arbitrary outcomes there is no obvious choice for an index of loss aversion, and it seems impossible to define a unique concept for a loss aversion index that is applicable to every situation. Nevertheless, the main theorem in (Köbberling \& Wakker, 2002c, see Chapter 5) that showed how the loss aversion of different agents can be compared through observed choices, can be generalized to general outcome sets.

\subsection{Comparative Loss Aversion}

Henceforth, $\succcurlyeq 1$ and $\geqslant$ denote the preferences over $L(X)$ of two agents who can be modelled through prospect theory with utility functions $U_{1}, U_{2}$ and weighting functions $w_{1}^{+}, w_{1}^{-}, w_{2}^{+}, w_{2}^{-}$. The preferences are assumed to coincide on $X$ and to both have reference

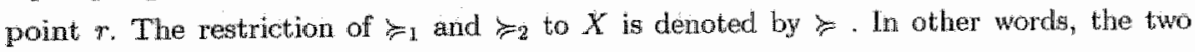
agents order the sure outcomes in the same way, but might have different preferences over lotteries. A lottery $P=\left(p_{1}, x_{1} ; \ldots ; p_{n}, x_{n}\right)$ is mixed if it yields a gain $x_{i} \succ r$ and also a loss $x_{j} \prec r$ with positive probability. A lottery which is not mixed is pure. 
Theorem 38 Assume that $X$ is rich enough such that it contains at least four outcanes $\bar{x}, \underline{x}, \bar{y}, \underline{y} \in X$ with $\bar{x}>\bar{y}>r>\underline{y}>\underline{x}$. The following two statements are equivalent:

(1) For all pure lotberies $P, Q \in L(X)$

$$
P \succcurlyeq Q \Leftrightarrow P \geqslant 2
$$

and for all mixed lotheries $R \in L(X)$

$$
R \geqslant 2 r \Rightarrow R \geqslant 1
$$

(ii) For all $p \in[0,1]$

$$
w_{1}^{*}(p)=w_{2}^{*}(p) \text { and } w_{1}^{-}(p)=w_{2}^{-}(p)
$$

and $U_{2}$ can be scoled such that there exists a $\gamma>1$ with

$$
U_{2}(x)= \begin{cases}U_{1}(x) & \text { for } x \geqslant r \\ \gamma U_{1}(x) & \text { for } x \prec r\end{cases}
$$

If some scaling convention is fixed to separate basic utility and loss aversion such that

$$
U_{1}(x)=\left\{\begin{array}{cc}
u_{1}(x) & \text { for } x \neq r \\
\lambda_{1} u_{1}(x) & \text { for } x \prec r
\end{array}\right.
$$

and if Theorem 38.(ii) is satisfied, then

$$
U_{2}(x)=\left\{\begin{array}{cc}
u_{1}(x) & \text { for } x \geqslant r \\
\gamma \lambda_{1} u_{1}(x) & \text { for } x<\pi
\end{array}\right.
$$

In other word, if Theorem 38. (38) is satisfied, a possible interpretation is that the corresponding valuetion function $C P T_{1}$ and $C P T_{2}$ difter only in loss aversion and agent 2 is more loss averse than agent 1 . The theorem exactly describes when two agents differ only in losis aversion without using a concrete loss aversion index. If two agents differ only in loss aversion, then implication (it) $\rightarrow(b)$ shows that the preferences over pure gain or pure loss lotteries coincide, but that every mixed lottery that is preferred to the reference point 
by the more loss averse agent is also preferred by the less loss averse. The proof of this implication is immediate. The reverse implication also holds and is more difficult to prove (see the Appendix of the chapter). The assumptions in Theorem 38 seem rather restrictive, but there is empirical evidence that no significant differences are found for pure gainor loss-lotteries, but differences do occur for mixed lotteries (Barberis, Huang, \& Santos 2001 , p. 17). Alternatively, the reader may think of only one agent, who's preferences are considered at two different time points; intermediate experiences in losses changed the loss aversion of that agent, whereas all other aspects did not change significantly.

The above theorem is a generalization of the main theorem in Kobberling \& Wakker (2002c, see Chapter 5). It relaxes the assumptions on the outcome set $X$. Theorem 38 . (i) requires that the preferences over pure lotteries of the two agents are the same. The more restrictive theorem in Kobbberling \& Wakker (2002c, see Chapter 5) only required equivalence of gain and loss acceptance sets, which is, because of the existence of certainty equivalents, for the special case of the real numbers virtually the same as requiring the same preferences over pure lotteries.

\subsection{Conclusion}

So far, loss aversion has been a well-established empirical phenomenon, but not much theoretical work has been devoted to it. $\mathrm{Ht}$ is a common understanding thet under prospect theory loss aversion is covered in the utility function. However, for general outcomes it is an open problem how to specify a basic utility underlying the observable utility that is not distorted by loss aversion. Nevertheless, Theorem 38 characterizes in terms of preferences what it means that one agent is more loss averse than another, if the two agents differ only in loss aversion. The two major contributions of this chapter are that it points out the difficulties in modeling loss aversion for general outcomes, and that it generalizes the main theoren in (Köbberling \& Wakker, 2002c, see Chapter 5).

\subsection{Appendix}

Proof of Theorem 38: The implication from (ii) $\rightarrow$ (i) is easy to see, so only the implication $(i) \rightarrow($ ii $)$ will be proven. Assume (i) is fulfilled. First it will be shown that $w_{1}^{+}(p)=w_{2}^{+}(p)$ for all $p \in[0,1]$. For that purpose the following auxiliary lemma is needed. 
Lemma 39 Assume that Theorem 38.(i) is fulfilled, and assume that $\bar{x}, \underline{x}, \bar{y}, \underline{y} \in X$ exist with $\succ \bar{y} \succ r ; \underline{y} \succ \underline{x}$. Then,

$$
w_{1}^{+}(p)-w_{1}^{+}(q)=w_{1}^{+}(s)-w_{1}^{+}(t) \Leftrightarrow w_{2}^{+}(p)-w_{2}^{+}(q)=w_{2}^{+}(s)-w_{2}^{+}(t)
$$

for all $p, q, s, t \in[0,1]$.

Proof: Assume that

$$
w_{1}^{+}(p)-w_{1}^{+}(q)=w_{1}^{+}(s)-w_{1}^{+}(t)
$$

Without loss of generality is can be assumed that $p \geq s \geq q \geq t$.

Case 1: If $w_{1}^{+}(q) \leq w_{1}^{+}(t) \frac{V_{2}(\vec{z})}{U_{5}(\bar{T})}$ then $\left(q, \overline{x_{i}}, 1-q, r\right) \geqslant 1(t, \bar{y} ; 1-t, r)$ and $(q, \bar{y} ; 1-q, r) \leqslant 1$ $(t, \bar{x} ; 1-t, r)$, i.e. it is possible to find $k, l \in[0,1]$ such that

$$
(q-k, \bar{x} ; k, \bar{y} ; ;-q, r) \sim_{1}(l, \bar{x} ; t-l, \bar{y} ; \mathbb{1}-t, r) .
$$

Therefore,

$$
\left(q-k ; \bar{x}_{;} ;, \bar{y}_{;} 1-q, r\right) \sim_{2}\left(l, \bar{x} ; t-l, \bar{y}_{;} 1-t, r\right)
$$

Indifference $(6.2)$ implies

$w_{1}^{+}(q-k)\left[U_{1}(\bar{x})-U_{1}(\bar{y})\right]+w_{1}^{+}(q) U_{1}(\bar{y})=w_{1}^{+}(l)\left[U_{1}(\bar{x})-U_{1}(\bar{y})\right]+w_{1}^{+}(t) U_{1}(\bar{y})$.

Together" with (6.1) we get

$w_{1}^{+H}(q-h)\left[U_{1}(\bar{x})-U_{1}(\bar{y})\right]+w_{1}^{+}(p) U_{1}(\bar{y})=w_{1}^{+}(l)\left[U_{1}(\bar{x})-U_{1}(\bar{y})\right]+w_{1}^{+}(s) U_{1}(\bar{y})$, i.e.

$(q-k, \bar{x} ; p-q+k, \bar{y} ; 1-p, r) \sim_{1}(l, \bar{x} ; s-l, \bar{y} ; 1-s, r)$. This implies

$(q-k, \bar{x} ; p-q+k, \bar{y} ; 1-p, r) \sim_{2}\left(l, \bar{x}_{i} s-l, \bar{y} ; 1-s, r^{r}\right)$, i.e.

$$
\left.w_{2}^{+}(q-k)\left[U_{2}(\bar{x})-U_{2}(\bar{y})\right]+w_{2}^{+}(p) U_{2}(\bar{y})=w_{2}^{+}(l)\left[U_{2}(\bar{x})-U_{2}(\bar{y})\right]+w_{2}^{+}(s) U_{2}(\bar{y})\right) .
$$

Equation (6.3) implies

$$
\left.w_{2}^{+}(q-k)\left[U_{2}(\bar{x})-U_{2}(\bar{y})\right]+w_{2}^{+}(q) U_{2}(\bar{y})=w_{2}^{+}(l)\left[U_{2}(\bar{x})-U_{2}(\bar{y})\right]+w_{2}^{+}(t) U_{2}(\bar{y})\right),
$$

i.e. (6.4) and (6.5) yield $w_{2}^{+}(p)-w_{2}^{+}(q)=w_{2}^{+}(s)-w_{2}^{+}(t)$, which we wanted to prove.

Case 2: Assume that $w_{1}^{+}(q)>w_{1}^{+}(t) \frac{V_{1}(x)}{U_{1}(i)}$. We define $t^{0}=t$ and $p^{0}=p$. Successively we will construct $t^{n}, p^{n} \in[0,1]$ for $n \in \mathbb{N}$ with $q \geq t^{n} \geq t, p \geq p^{n} \geq s$,and $w_{1}^{+}\left(p^{n}\right)-w_{1}^{t}(q)=$ 


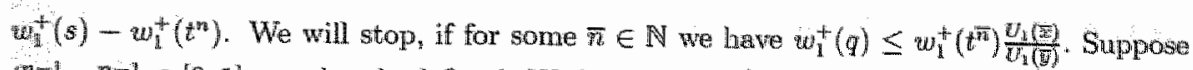
$t^{n-1}, p^{n-1} \in[0,1]$ are already defined. We have $q \geq t^{m-1} \geq t, p \geq p^{n-1} \geq s$, and

$$
w_{1}^{+}\left(p^{n-1}\right)-w_{1}^{+}(q)=w_{1}^{+4}(s)-w_{1}^{+}\left(t^{n-1}\right) .
$$

If $w_{1}^{+}(q)>w_{1}^{+}\left(t^{n-1}\right) \frac{W_{1}(\bar{x})}{U_{1}(\bar{y})}$, we choose $t^{n}$ such that $w_{1}^{+}\left(t^{n}\right)=w_{1}^{+}\left(t^{n-1}\right) \frac{U_{1}(x)}{U_{1}(\bar{y})}$ and $p^{n i}$ such that

$$
w_{1}^{+}\left(p^{n-1}\right)-w_{1}^{+}\left(t^{n}\right)=w_{1}^{+}\left(p^{n}\right)-w_{1}^{+}\left(t^{n-1}\right) .
$$

It follows that $q \geq t^{n} \geq t^{n-1}$ and $p^{n-1} \geq p^{n} \geq s$. Because $w_{1}^{+}\left(t^{n}\right)=w_{1}^{+}\left(t^{n-1}\right) \frac{U_{1}(\tilde{s})}{U_{1}(\bar{y})}$ we can infer as in Case 1 that

$$
w_{2}^{+}\left(p^{n-1}\right)-w_{2}^{+}\left(t^{n}\right)=w_{2}^{+}\left(p^{n}\right)-w_{2}^{+}\left(t^{n-1}\right)
$$

From (6.6) and (6.7) we have $w_{1}^{+}\left(p^{n^{n}}\right)-w_{1}^{+}(q)=w_{1}^{+}(s)-w_{1}^{+}\left(t^{n}\right)$.

If $w_{1}^{+}(q) \leq w_{1}^{+}\left(t^{\bar{n}}\right) \frac{v_{1}(\bar{x})}{U_{1}(\bar{y})}$ for some $\bar{n} \in \mathbb{N}$, then we can infer as in Case 1 that

$$
w_{2}^{+}\left(p^{\bar{n}}\right)-w_{2}^{+}(q)=w_{2}^{+}(s)-w_{2}^{+}\left(t^{\bar{n}}\right)
$$

Because (6.8) holds for all $0 \leq m \leq \pi,(6.8)$ and $(6.9)$ imply $w_{2}^{+}(p)-w_{2}^{+}(g)=w_{2}^{+}(s)-w_{2}^{+}(t)$, which we wanted to prove.

It is left to show that for some $\bar{n} \in \mathbb{N}$ we have $w_{1}^{+}(q) \leq w_{1}^{+}\left(t^{\bar{n}}\right) \frac{V_{1}(\bar{x})}{U_{1}(\bar{x})}$. For all of the constructed $t^{n}$ we have $w_{1}^{+}\left(t^{n}\right) \frac{U_{1}(\bar{g})}{U_{1}(\bar{y})}=w_{1}^{+}\left(t^{n}\right)\left(\frac{U_{1}(\bar{x})}{U_{1}(\overline{\bar{D}})}\right)^{n}$. Because $\frac{U_{1}(\overline{\bar{x}})}{U_{1}(\bar{y})}>1$, there has to exist an $\bar{n} \in \mathbb{N}$ with $w_{1}^{+}(q) \leq w_{1}^{+}\left(t^{\bar{n}}\right) \frac{U_{1}(\bar{x})}{U_{1}(\overline{\bar{x}})}=w_{1}^{+}\left(t^{0}\right)\left(\frac{U_{1}(\bar{x})}{U_{1}(\overline{\bar{q}})}\right)^{\bar{\pi}}$, and the proof is complete.

The proof of the theorem will now continue. Suppose there exists a $p^{0} \in(0,1)$ such that $w_{1}^{+}\left(p^{0}\right) \neq w_{2}^{+}\left(p^{0}\right)$. Without loss of generality it is assumed that $w_{1}^{+}\left(p^{0}\right)>w_{2}^{+}\left(p^{0}\right)$. Because of continuity of $u_{1}^{+}$and $w_{2}^{+}$there have to exist $p^{1}<p^{0}<p^{2}$ such that

$$
w_{1}^{+}\left(p^{1}\right)=w_{2}^{+}\left(p^{1}\right), w_{1}^{+}\left(p^{2}\right)=w_{2}^{+}\left(p^{2}\right)
$$

and for all $p \in\left(p^{1}, p^{2}\right)$

$$
w_{1}^{+}(p)>w_{2}^{+}(p)
$$


Next, $p^{3} \in\left(p^{1}, p^{2}\right)$ is chosen such that $w_{1}^{+}\left(p^{2}\right)-w_{1}^{+}\left(p^{3}\right)=w_{1}^{+}\left(p^{3}\right)-w_{1}^{+}\left(p^{1}\right)$. By the axwitury Lemma $w_{2}^{+}\left(p^{2}\right)-w_{2}^{+}\left(p^{3}\right)=w_{2}^{+}\left(p^{3}\right)-w_{2}^{+}\left(p^{\mathbb{1}}\right)$. Together with $(6.10)$ this implies $w_{k}^{+}\left(p^{3}\right)=w_{2}^{+y^{+}}\left(p^{3}\right)$. But this is a contradiction to (6.11). It follows that $w_{1}^{+}(p)=w_{2}^{+}(p)$ for al $p \in[0,1]$.

Analogously, it can be shown that $w_{1}^{-}(p)=w_{2}^{-}(p)$ for all $p \in[0,1]$.

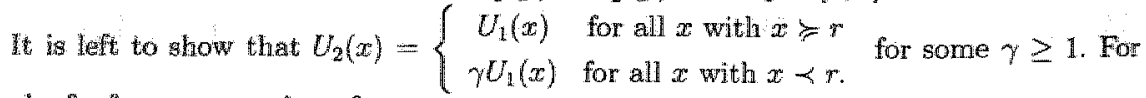
all $x^{2}, x^{2}, x^{3} \in X$ with $x^{1} \succ x^{2} \succcurlyeq r$ a $p \in[0,1]$ can be found such that

$$
\left(p, x^{1} ; 1-p, r\right) \sim_{1} x^{2}
$$

and therefore

$$
\left(p, x^{1} ; 1-p, r\right) \sim_{2} x^{2}
$$

For $(6.12)$ we have $U_{1}\left(x^{2}\right)=w_{1}^{+}(p) U_{1}\left(x^{1}\right)$ and from $(6.13)$ we have $U_{2}\left(x^{2}\right)=w_{2}^{+1}(p) U_{2}\left(x^{1}\right)$. Because $w_{1}^{+4}(p)=w_{2}^{+}(p)$ it follows that $\frac{U_{1}\left(x^{4}\right)}{U_{1}\left(x^{2}\right)}=\frac{U_{y}\left(x^{1}\right)}{U_{2}\left(x^{2}\right)}$. The latter equation holds for all $x^{1}, x^{2} \in X$ with $x^{1}, x^{2} \succcurlyeq r$.

This implies that $U_{2}(x)=\alpha U_{1}(x)$ for all $x \in X$ with $x \succcurlyeq r$ and some $\alpha>0$. Analogously, it can be shown that $U_{2}(x)=\gamma U_{1}(x)$ for all $x \in X$ with $x \leqslant r$ and some $\gamma>0$.

Let $q \in[0,1]$ be such that

$$
(q, \bar{x} ; 1-q, \underline{x}) \sim_{2} r
$$

Then

$$
w_{2}^{+}(q) U_{2}(\bar{x})+w_{2}^{-}(1-q) U_{2}(\underline{x})=w_{1}^{+}(q) \alpha U_{1}(\bar{x})+w_{1}^{-}(1-q) \gamma U_{1}(\underline{x})=0
$$

Indifference (6.14) implies $(q, \bar{x} ; 1-q, \underline{x}) \succcurlyeq_{1} r$, i.e.

$$
w_{1}^{+1}(q) U_{1}(\ddot{x})+w_{1}^{-\mu}(1-q) U_{1}(\underline{x}) \geq 0
$$

The two equations (6.15) and (6.16) imply $a \leq \gamma$. Because $U_{2}$ is unique up to scale (Wakker \& Tversky, 1993), it is possible to choose $a=1$, which implies $\gamma \geq 1$, and the proof is complete. 


\section{Chapter 7}

\section{The Effect of Decision Weights in}

\section{Bargaining Problems}

Bargaining problems are considered where the preferences of the bargainers deviate from expected utility but can be modelled according to rank-dependent utility theory. Under rank-dependent utility two factors influence the risk attitude of a decision maker: the utility function and the probability weighting function. Arising from the same definition of risk aversion, two forms of risk aversion can be distinguished: utility risk aversion and probabilistic risk aversion. The main finding is that these two forms of risk aversion can have surprisingly opposite consequences for bargaining solutions that exhibit a weak monotonicity property. In particular, in a large class of bargaining problems both, increased utility risk aversion and decreased probabilistic risk aversion of the opponent, are adwantageous for a player. This is demonstrated for the Kalai-Smorodinsky bargaining solution. The Nash bargaining solution does not behave regularly in this respect. ${ }^{\text {" }}$

\subsection{Introduction}

A bargaining problem is described by a set of potential outcomes, including a so-catled disagreement outcome. The bargaimers try to reach agreernent on one of the outcomes or on a lottery between these outcoines. If they fail, the disagreement outcome results. A bargaining solution suggests an agreement outcome or lottery for each possible bargaining problem. In this chapter, the somcalled welfarist approach to bargaining is adopted. In

\footnotetext{
The results in this clapter were first formalated in (Kobberlinge \& Paters, 2002 \%.
} 
this approach, the solution to the bargaining problem depends exclusively on its image in utillty space.

The axiomatic approach to two-person bargaining problems was initiated by Nast (1950), wnder the assumption that the two bargainers maximize expected utility. In ex: pected utility, risk aversion is an important empirical phenomenon. Yaari (1969) provides an intuitive definition of risk aversion and shows that under expected utility one decision maker is more risk averse than another if and only if the utility function of the former is a strictly increasing concave transformation of that of the latter. An interesting question, first studied by Kamnai (1977) and Khlstrom, Roth, \& Schmeidler (1981), concerms the effect of risk aversion on the outcomes predicted by bargaining solutions. Their answers to this question confirm a plausible intuition: it is advantageous to play against a more risk. averse bargainer. These results are driven by the fact that a more risk averse bargaines has a more concave utility function. Therefore, utility at low levels increases at a faster rate and satisfaction may appear faster. Because most bargaining solutions are sensitive to this, a more risk averse bargainer may be easier to satisfy, which is advantageous for the opponent (cf. Peters, 1992).

Expected utility as a normative basis for decision making is appealing, but as empirical findings show, it is descriptively unsatisfactory. Therefore, alternative models have been developed of which rank-dependent utility (Quiggin, 1982), which extends expected utility by allowing for distortion of probabilities, is the best known.

This chapter considers bargaining problems where the bargainers' preferences can be represented by rank-dependent utility. In this model Yaari's concept of increased risk aversion has still the same natural meaning: a more risk averse person prefers less lotteries over each riskless altemative. In contrast with expected utility, however, not only the utility function but also a probability weighting function determines the risk attitude of at decision maker. An elegant and simple characterization of increased risk aversion as in the case of expected utility is not yet avallable. Chateauneuf \& Cohen (1994) provide some partial results. Chew, Karni, \& Safra (1987) characterize aversion to mean-preserwing spreads, which is more restrictive than risk aversjon.

In the present clapter, two factors of risk aversion are distinguished: utility risk awersion (as in the expected utility model), associated with the utility function; and probabilistic risk aversion, associated with the probability weighting function. In our application to bargaining we concentrate on the latter, because the results for the former are closely 
reluted to those in expected utility. As in the related literature, the central question is: is ut advantageous or disadvantageous to bargain with a mone risk averse person? In Section 7.3, we investigate the impact of both, utility and probabilistic risk aversion, for the KalaiSmorodinsky bargaining solution. (Kalai \& Smorodinsky, 1975). The main finding is that in a large class of bargaining problems, it is advantageous to have a less probabilistically risk averse, or a mone utility risk averse opponent. The known effect of risk aversion for the expected utility case (cf. Kihlstrom et al., 1981) is a special case of the latter result. Since both forms of risk aversion arise from the same concept of comparative risk aversion, this contrast is rather surprising. On closer inspection, however, it is not counterintuitive that increased probabilistic risk aversion of the opponent could be bad for a player, because such an opponent might insist on larger probabilities for good alternatives and thereby redruce the other player's utility.

The two results also have some strategic implications, if it is assumed that bargainers can be dishonest about their true preferences and pretend to evaluate lotteries by means of a different utility or probability weighting function. Specifically, it does not pay to pretend to be less probabilistically risk averse, or more utility risk averse, but it might sometimes pay to pretend to be more probabilistically or less utility risk averse. Fon the expected utility case related papers in this direction are Crawford \& Varian (1979) and Sobel (1981, 2001).

Although our focus is on the Kalai-Smorodinsky solution the main results in this chapter can be extended to solutions that exhibit some form of monotonicity. Typically, these do not include the Nash bargaining solution. If this solution is used, a more probabilistically risk averse opponent might be better but also worse for a player. We justify the use of the Kalai-Smorodinsky solution in bargaining under rank-dependent utility by showing that the axiomatization of Kalai \& Smorodinsky (1975) still makes sense (see Section 7.4).

Axiomatic bargaining without expected utillity has been studied in other articles (Tubinstein, Safra, \& Thomson, 1992; Safra \& Zilcha, 1993; Satre, Zhou, \& Zilcha, 1990; Burgos, Grant, \& Kajii, 2002; and Volij \& Winter, 1999). The paper of Volij \& Winten (1999) is closest to the present chapter. It applies Yaari's dual theory of choice to bargainung problems where the set of altermatives consists of all distributions of ane wnit of perfectly diwisible good, and wises the stronger notion of aversion to mean-preservinus spreads to study changes in the Nash solution. Shalev (1997) has studied the eflect of loss aversion-see also Section 7.5. The proofs are collected in the Appendix of the chapter. 


\subsection{Preliminaries}

\subsubsection{Rank-dependent Utility}

Throughont, the set; $A$ of rishless alternatives is a nonempty compact topological space. A lottery is a probability measure with finite support on $A$, typically denoted by $P=$ $\left(p^{1}, a^{1}, \ldots ; p^{n}, a^{n}\right)$ where $a^{1}, \ldots, a^{n} \in A, p^{i} \geq 0$ for aill $i$ and $\sum^{n} p^{i}=1$. The set of all lotteries is $L(A)$. A riskless alternative $a \in A$ is identified with the lottery $(1, a) \in L(A)$ :

A wtility function $U: A \rightarrow \mathbb{R}$ is a continuous function that assigns to each riskless alterintive a real number, expressing its value for a decision maker. A weighting function is a continuous, strictly increasing function $w:[0,1] \rightarrow[0,1]$ with $w(0)=0$ and $w(1)=1$, representing the decision maker's personal appraisal of probabilities. For a decision maker with utility function $U$, the preferences over $L(A)$ can be nodelled by rank-dependent

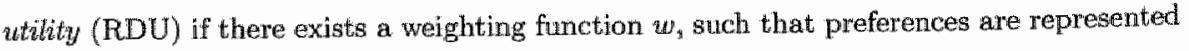
by the functional $R D U: L(A) \rightarrow \mathbb{R}$ defined by

$$
\begin{aligned}
R D U(P)= & w\left(p^{\rho(1)}\right) U\left(a^{\rho(1)}\right)+ \\
& \left.\sum_{i=2}^{n} \mid w\left(p^{\rho(1)}+\cdots+p^{\rho(i)}\right)-w\left(p^{\rho(1)}+\cdots+p^{\rho(i-1)}\right)\right] U\left(a^{\rho(i)}\right),
\end{aligned}
$$

where $\rho$ is a permutation that orders the rimkless alternatives in the lottery from best to worse, i.e., $U\left(a^{\mu(1)}\right) \geq \ldots \geq U\left(a^{\rho(n)}\right)$ (cf. Quiggin, 1982). Expected utility holds if $w(p)=p$ for all $p \in[0,1]$.

Rank-dependent utility, in contrast to exprected utiljty, permits transformations of probabilities. An alternative is weighted by the transformed probability of obtaining the alternative or something better mimus the transformed probability of obtaining something better.

\subsubsection{Comparative Risk Aversion}

Yari (1969) introduced a natural definition of risk aversion. A decision maker is more risk averse than another one, if whenever the former (weally) prefers a lottery to a riskless alternative the latter also does, In expected utility it is well known result that one decision maker is more risk averse than a second one if the utility function of the first is atrictly increasing concave transformation of that of the second. Under rank-dependent 
utilitity, a second component influences the risk attitude, namely, the weighting function. In the lemma below and in the rest of the chapter we consider these two components separately, by keeping one fixed and varying the other.

Definition 40 Let the preferences over $L(A)$ of two decision makers (DM and $\widetilde{D M}$ ) be represented by $R D U$-functionals $R D U$ and $\widetilde{R D U}$. $\widetilde{D M}$ is more risk averse than $D M$ if for all $a \in A$ and all $P \in L(A)$ with $\widetilde{R D U}(P) \geq \widetilde{R D U}(a)$ also $R D U(P) \geq R D U(a)$.

Lemma 41 Let the preferences over $L(A)$ of $D M$ and $\widetilde{D M}$ be represented by $R D U$ and $\widehat{R D U}$ with utility functions $U$ and $\widetilde{U}$ and weighting functions $w$ and $\widetilde{U}$, respectively.

1. If $w=\widetilde{w}$ then $\widetilde{D M}$ is more risk averse than $D M$ if and only if there exists a concave strictly increasing function $k: \mathbb{R} \rightarrow \mathbb{R}$ such that $\widetilde{U}=k \circ U$.

2. If $U=\alpha \widetilde{U}+\beta$ for $\alpha, \beta \in \mathbb{R}, \alpha>0$ then $\widetilde{D M}$ is more rist averse than $D M$ if $\bar{w}(p) \leq w(p)$ far all $p \in[0,1]$. In particular, if $U=\widetilde{U}$ this implies that $\widehat{R D U}(P) \leq$ $R D U(P)$ for all $P \in L(A)$.

If $A$ is connected, the "only if" implication in part 2 of Lemma 41 also holds. In the situation of Lemma 41 , part 1 , we say that decision maker $\widetilde{D M}$ is more utility risk averse than decision maker DM; whereas in the situation of Lemma 41 , part 2 , we say that $\widehat{D M}$ is mone probabilistically risk averse than $D M$. Although the above result is well known and simple to prove, it was not yet stated as in Lenma. 4.1. Part 1 of the lemma is a generalization of Yaari's result (1969) for expected utility; a predecessor of part 2 can be found in Yaari (1986). A proof can also be deduced from Chateauneuf \& Cohen (1994). A general characterization of comparative risk aversion that allows for varying utility and weighting functions at the same time, is not yet available.

\subsubsection{Bargaining Problems and Solutions}

As before, a nonempty compact topological space $A$ is the set of riskless alternativen. Let $R D U_{1}$ and $R D U_{2}: L(A) \rightarrow \mathbb{R}$ be the RDU-functionals of two bargainers with utility functions $U_{1}$ and $U_{2}$ and weighting functions $w_{1}$ and $w_{2}$, respectively. A designated element $\bar{a} \in A$ is the disagrement altemative. We assume throughout that there is a lottery $P \in L(A)$ with $R D U_{1}(P)>U_{1}(\bar{a})$ and $R D U_{2}(P)>U_{2}(\bar{a})$. The two bargainers try to 
reach an agreenent in $L(A)$. If they fail, $\bar{a}$ results. The quadruple $\left(A, \bar{a}, R D U_{1}, R D U_{2}\right)$ is called a (two-person) bargaining problem $\mathbb{B}$ is the set of all bargaining problems.

For a bargaining problem $B=\left(A, \bar{\alpha}_{1} R D U_{1}, R D U_{2}\right)$, define

$$
\begin{aligned}
& S(B)=\operatorname{cl}\left\{\left(R D U_{1}(P), R D U_{2}(P)\right): P \in L(A)\right\} \text { and } \\
& d(B)=\left(U_{1}(\bar{a})_{9} U_{2}(\bar{a})\right)
\end{aligned}
$$

where 'cl' denotes the (topological) closure. The point $d(B)$ is called the disagreement outcome. The set $S(B)$ is called the feasible set of $B$. It is a compact subset of $\mathbb{R}^{2}$. In contrast to the case of bargaining problems under expected utility, examples (with infinite A) can be constructed where the set $\left\{\left(R D U_{1}(P), R D U_{2}(P)\right): P \in L(A)\right\}$ is not closed.

We define

$$
\mathbb{S}=\{(S, d): S=S(B) \text { and } d=d(B) \text { for some } B \in \mathbb{B}\}
$$

A bargaining solution is a map $F: S \rightarrow \mathbb{R}^{2}$ that assigns to each $(S, d) \in \mathbb{S}$ an element of $S$. In the rest of the chapter, we also write $F(B)$ instead of $F(S(B), d(B))$.

\subsubsection{Feasible Sets of Bargaining Problems}

Under expected utility, the set of all possible feasible sets is the set of all compact convex sets in $\mathbb{R}^{2}$. Because of continuity of the weighting functions, a feasible set $S(B)$ is connected under rank-dependent utility, but it is not necessarily convex. It does, however, satisfy a weaker form of convexity, as formulated in the following lemma.

For any points $s$ and $t$ in $\mathbb{R}^{2}$ denote by $[s, t]$ the straight line segment with $s$ and $t$ as endpoints, i.e., the convex hull of $s$ and $t$. The vector inequality $t>s$ means $t_{i}>s_{i}$ for $i=1,2$, and $t \geq s$ means $t_{i} \geq s_{i}$ for $i=1,2$.

Lemma 42 Let $(S, d) \in \mathbb{S}$. Then for all $s, t \in S$ with $s_{1}=t_{1}$ or $s_{2}=t_{2}$ we have $[s, t] \subseteq S$.

This lemma implies in particular that a feasible set $S$ is not only connected but also simply connected: it contains no 'holes' (cf. Lemma 52 in the Appendix of the chapter).

For $(S, d) \in \mathbb{S}$ the Pareto optimal set $P(S)$ is defined by

$$
P(S)=\{s \in S: \text { there exists no } t \in S \text { with } t \geq s \text { and } t \neq s\}
$$


Insitead of $P(S(B))$ we also write $P(B)$.

The following lemma implies that the Pareto optimal set is connected.

Lemma 43 Let $(S, d) \in \mathbb{S}$. Then there is a closed interval $I \subset \mathbb{R}$ and a continuous strictly decreasing function $f: I \rightarrow \mathbb{R}$ such that $P(S)$ is the graph of $f$, i.e.

$$
P(S)=\{(t, f(t)): t \in I\}
$$

Lermma 43 says that Pareto optimal subset of a feasible set $S$ behaves nicely: there are no indentations nor even horizontal or vertical line segments. Analogous results can be derived for the other parts of the boundary of $S$ (cf. Lemma 54 in the Appendix of the chapter). Lemma 42 implies that also the possibly flat parts in the north, east, south, and west cannot have indentations.

The next lemma provides a partial answer to the converse question: Which nonempty compact subsets $S$ of $\mathbb{R}^{2}$ can be gemerated as feasible sets of bargaining problems under rank-dependent utility? The lemma says that every connected compact Pareto optimal set can be generated by an underlying bargaining problem.

Lernma 44 Let $I \subset \mathbb{R}$ be a nonempty closed interval and let $f: I \rightarrow \mathbb{R}$ be a contimuous strictly decreasing function. Then there is a $B \in \mathbb{B}$ such that $P(B)$ is the graph of $f$, i.e.

$$
P(B)=\{(t, f(t)): t \in I\}
$$

\subsubsection{The Kalai-Smorodinsky Bargaining Solution}

For $(S, d) \in \mathbb{S}$, the utopia pornt is defined by:

$$
u(S, d)=\left(\max \left\{s_{1}: s \in S, s \geq d\right\}, \max \left\{s_{2}: s \in S, s \geq d\right\}\right)
$$

For $B \in \mathbb{B}$ we also write $u(B)$ instead of $u(S(B), d(B))$. The Kalai-Smorodinsky solution $K S$ (Kallai \& Smorodinsky, 1975) assigns to each $(S, d) \in \mathbb{S}$ the maximal point of $S$ on the line segment with $d$ and $u(S, d)$ as endpoints. It is well defined because $S$ is compact. By Lemma $43, K S(S, d)$ is a Pareto optimal point for every $(S, d) \in \mathbb{S}$.

This is an adaptation of the definition of KS for expected utility to rank-dependent utility. Under expected utility, the Kalai-Smorodinsky solution can be justified and char- 
acterized by a set of axioms (Kalai \& Smorodinsky, 1975). This axiomatization can be extended to the rank-dependent utility case, as we will show in Section 7.4 below.

\subsection{Sensitivity of the KS Bargaining Solution to Risk}

We investigate the sensitivity of the Kalai-Smorodinsky bargaining solution to changes in the risk attitude of the bargainers. We first assume that both bargainers weakly prefer all riskless alternatives to the disagreement alternative, a condition which will be weakened in Section 7.3.4. We examine the changes in the outcome suggested by the Kalai-Smorodinsky bargaining solution if the risk aversion of one of the players changes. First, we keep the weighting function fixed and vary the utility function; second, we keep the utility function fixed and vary the weighting function. The first case is a generalization to rank-clependent utility of known results for expected utility. Define

$$
\mathbb{B}^{*}=\{B \in \mathbb{B}: s \geq d(B) \text { for all } s \in S(B)\}
$$

\subsubsection{Utility Risk Aversion}

Theorem 45 Let $B$ and $C$ be bargaining problems in $\mathbb{B}^{+}$that are identical except that player 2 is more utility risk averse in $C$ than in $B$. Then $K S_{1}(B) \leq K S_{1}(C)$.

The theorem says that a player does not lose when bargaining with a (new) more utility risk averse opponent. Figure 7.1(a) illustrates this. In the picture we use the fact that under rank-dependent utility, the utility function is unique up to scale and location and the Kalai-Smorodinsky solution satisfies scale invariance (see Section 7.4), so player 2 's utility function in bargaining problem $C$ can be scaled such that $d(B)=d(C)$ and $u(B)=u(C)$.

\subsubsection{Probabilistic Risk Aversion}

Theorem 46 Let $B$ and $C$ be bargaining problems in $B^{+}$that are identical except that ployer 2 is more prababilistically risk averse in $C$ than in $B$. Then $K S_{1}(B) \geq K S_{1}(C)$.

Theorem 46 says that a player does not profit from bargaining with a (new) more probabilistically risk averse opponent. This is illustrated in Figure 7.1(b), where the 


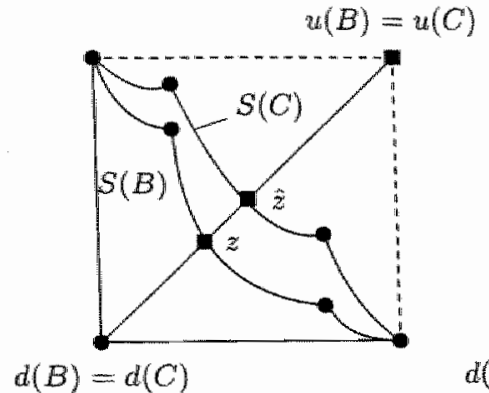

(a)

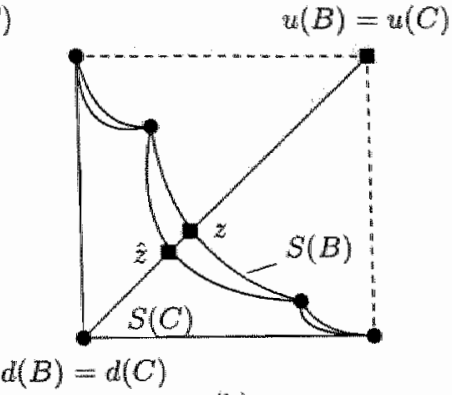

(b)

Figure 7.1: Diagram (a) illustrates Theorem 45 and diagram (b) illustrates Theorm 46. In both diagrams, $z=K S(B)$ and $\hat{z}=K S(C)$. The solid dots correspond to riskless altematives.

utility functions of players 2 in $B$ and $C$ are taken to be equal (cf. Lemma 41 , part 2, and recall that $\mathrm{KS}$ is scale invariant).

Observe that both in Theorem 45 and in Theorem 46 the players 2 in $B$ and $C$ are different players, so that neither these results nor the associated diagrams imply anything about gains or losses of these players. See also the discussion below.

\subsubsection{Discussion and Strategic Implications}

Although utility risk aversion and probabilistic risk aversion have a similar impact on the preferences of a decision maker, namely, the more utility or probabilistically risk averse a player is, the less lotteries are preferred over each sure alternative, they have contrary effects in bargaining problems with respect to the Kalai-Smorodinsky bargaining solution. An intuition for this is as follows. If a player can choose between a more and a less utility risk averse opponent, it is advisable to choose the more utility risk averse one, because the marginal utility of the more utility risk averse player diminishes faster, so that this player is easier to satisfy. But if a player cen choose between a more and a less probabilistically risk averse opponent, it is advisable to choose the less probabilistically risk averse one, because the more probabilistically risk averse player weighs probabilities for alternatives with high outcomes lower and therefore asks for more security for obtaining high outcomes.

These considerations represent a positive view: they are of interest for the case in 
which the Kalai-Smorodinsky solution offers a good description of the bargaining outcome. Additionally, it is interesting to consider the strategic implications of Theorems 45 and 46 for a bargainer under the assumption that preferences are private knowledge, so that it is possible to be dishonest about the true preferences. These implications are relevant from a normative point of view, in particular, for the question of mechanism design.

Let $B=\left(A, \bar{a}, R D U_{1}, R D U_{2}\right)$ be a bargaining problem, where $R D U_{2}$ corresponds to the true preferences of player 2 . We consider four cases, namely that player 2 pretends to be more or less utility or probabilistically risk averse. For simplicity, it is assumed for the rest of this subsection that $A$ is finite. This ensures that the image of $L(A)$ in $\mathbb{R}^{2}$ is closed and, therefore, it also ensures the existence of a lottery with rank-dependent utility imange equal to the point suggested by the Kalai-Smorodinsky solution. Nevertheless, analogons results can be derived for infinite $A$.

1. Does it make sense for player 2 to pretend to be more utility risk averse, with preferences represented by $\widehat{R D U}_{2}$ ?

Let $C=\left(A, \bar{a}_{3} R D U_{1}, \widehat{R D U}_{2}\right)$. We assume that $\widetilde{R D U}_{2}$ is scaled such that $d(B)=d(C)$ and $u(B)=u(C)$. Let $P, Q \in L(A)$ such that $K S(B)=\left(R D U_{1}(P), R D U_{2}(P)\right)$ and $K S(C)=$ $\left(R D U_{1}(Q), \widehat{R D U}_{2}(Q)\right)$. Theorem 45 implies that $R D U_{1}(P) \leq R D U_{1}(Q)$. If $R D U_{1}(P)<$ $R D U_{1}(Q)$, then Pareto optimality implies $R D U_{2}(P)>R D U_{2}(Q)$. If $R D U_{1}(P)=R D U_{1}(Q)_{*}$ then $R D U_{2}(P)=\widehat{R D U}_{2}(Q)$, because $d(B)=d(C)$ and $u(B)=u(C)$. But $\widetilde{R D U}_{2}(Q) \geq$ $R D U_{2}(Q)$ by Lemma 41 . Hence, in every case $R D U_{2}(P) \geq R D U_{2}(Q)$. Thus, player 2 never profits from pretending to be more utility risk averse.

2. Does it make sense for player 2 to pretend to be less utility risk averse, with preferences represented by $\widehat{R D U}_{2}$ ?

Nothing can be said in general here, as the following example shows.

Example 47 Let $\left.A=\left\{(0,0),(1,0),(0,1),\left(\frac{55}{100}, \frac{55}{100}\right)\right\}, \bar{A}=\left\{(0,0),(1,0),(0,1),\left(\frac{1}{2}, \frac{9}{10}\right)\right)\right\}$, $\bar{a}=(0,0), U_{i}(a)=a_{i}$ for $i \in\{1,2\}, \widehat{U}_{2}(0)=0, \widehat{U}_{2}\left(\frac{55}{100}\right)=0.2, \widehat{U}_{2}\left(\frac{9}{10}\right)=\frac{55}{100}, \widehat{U}_{2}(1)=1$, and $w_{1}(p)=w_{2}(p)=\widehat{w}_{2}(p)=p$ for all $p \in[0,1]$.

Let $B=\left(A, \bar{a}_{1} R D U_{1}, R D U_{2}\right)$ and $C=\left(A, \bar{a}_{2} R D U_{1}, \widehat{R D U_{2}}\right)$.

Moreover, let $\bar{B}=\left(\bar{A}, \bar{a}, R D U_{1}, R D U_{2}\right)$ and $\bar{C}=\left(\bar{A}_{1}, \bar{a}_{1} R D U_{1}, \widehat{R D U}_{2}\right)$. The lotteries that 
give rise to the Kalai-Smorodinsky solution are, respectively

$$
\begin{aligned}
& P=\left(1,\left(\frac{55}{100}, \frac{55}{100}\right)\right), \\
& Q=\left(\frac{1}{2},(1,0) ; \frac{1}{2},(0,1)\right), \\
& \bar{P}=\left(\frac{2}{7},(1,0) ; \frac{5}{7},\left(\frac{1}{2}, \frac{9}{10}\right)\right), \\
& \bar{Q}=\left(1 ;\left(\frac{55}{100}, \frac{55}{100}\right)\right) .
\end{aligned}
$$

We have

$$
R D U_{2}(P)=\frac{55}{100}>\frac{1}{2}=R D U_{2}(Q)
$$

but

$$
\operatorname{RDU}_{2}(\bar{P})=\frac{9}{14}<\frac{55}{100}=\operatorname{RDU}_{2}(\bar{Q})
$$

Hence, pretending to be less utility risk averse can be advantageous (player 2 in the pair $\bar{B}, \bar{C}$ ) but also disadvantageous (player 2 in the pair $B, C$ ).

3. Does it make sense for player 2 to pretend to be less probabilistically risk averse with preferences represented by $\widehat{R D U}_{2}$ ?

Let $C=\left(A, \bar{a}, R D U_{1}, \widehat{R D U}_{2}\right)$, and assume again that the utility function $\widehat{U}_{2}$ of player 2 is scaled such that $d(C)=d(B)$ and $u(C)=u(B)$, i.e., $\widehat{U}_{2}=U_{2}$. Let $P, Q \in L(A)$ such that $K S(B)=\left(R D U_{2}(P), R D U_{2}(P)\right)$ and $K S(C)=\left(R D U_{1}(Q), \widehat{R D U}_{2}(Q)\right)$. Theorem 46 implies that $R D U_{1}(P) \leq R D U_{1}(Q)$. If $R D U_{1}(P)<R D U_{1}(Q)$, then Pareto optimality implies $R D U_{2}(P)>R D U_{2}(Q)$. If $R D U_{1}(P)=R D U_{1}(Q)$, then $R D U_{2}(P)=\widehat{R D U}_{2}(Q)$, because $d(C)=d(B)$ and $u(C)=u(B)$. But $\widehat{R D V}_{2}(Q) \geq R D U_{2}(Q)$ by Lemma 41. Hence, in every case $R D U_{2}(P) \geq R D U_{2}(Q)$. Thus, player 2 never profits from pretending to be less probabilistically risk averse.

4. Does it make sense for player 2 to pretend to be more probabilistically risk averse, with preferences represented by $\widetilde{R D U}_{2}$ ?

Nothing can be said in general here, as the following example shows. 
Example 48 Let $A=\{(0,0),(1,0),(0,1)\}, \bar{A}=A \cup\left\{\left(\frac{55}{100}, \frac{55}{100}\right)\right\}, \bar{a}=(0,0), U_{i}(a)=a_{i}$ for all $a \in \bar{A}_{i} i \in\{1,2\}, w_{1}(p)=p_{2} w_{2}(p)=\sqrt{p}, w_{2}(p)=p$ for all $p \in[0,1]$. Let $B=$ $\left(A, \bar{a}_{4} R D U_{1}, R D U_{2}\right)$ and $C=\left(A, \vec{C}_{1}, R D U_{1}, \overline{R D U}_{2}\right)$, and let $\bar{B}=\left(\bar{A}_{1}, \bar{a}_{2} R D U_{1}, R D U_{2}\right)$ and $\bar{C}=\left(\bar{A}_{4}, \bar{a}, R D U_{1}, \widehat{R D U}_{2}\right)$. The lotweries that give rise to the Kalai-Smorodinsky solution in $B, C, \bar{B}$, and $\bar{C}$, are, respectively,

$$
\begin{aligned}
& P \approx(0.618,(1,0) ; 0.382,(0,1)), \\
& Q=\left(\frac{1}{2},(1,0) ; \frac{1}{2},(0,1)\right), \\
& \bar{P} \approx\left(0.401,(1,0) ; 0.241,(0,1) ; 0.298,\left(\frac{55}{100}, \frac{55}{100}\right)\right) \\
& \bar{Q}=\left(1,\left(\frac{55}{100}, \frac{55}{100}\right)\right) .
\end{aligned}
$$

We hive:

$$
R D U_{2}(P) \approx 0.618<0.707 \approx R D U_{2}(Q)_{4}
$$

but

$$
R D U_{2}(\bar{P}) \approx 0.625>\frac{55}{100}=R_{2 D U_{2}}(\bar{Q})
$$

Hence, pretending to be more probabilistically risk averse can be advantageous (player 2 in the pair $B, C$ ) but also disadvantageous (player 2 in the pair $\bar{B}, \bar{C}$ ).

\subsubsection{Extension from $\mathbb{B}^{+}$to $\mathbb{B}$}

In this subsection the restrictions in Theorem 40 on bargaining problems are weakened. Riskless alternatives are permitted that are worse than the disagreement alternative for one or both players. In the following, we only consider the effect of weighting functions. For results concerning utility risk aversion in the expected utility case, see Roth \& Rothblum (1982).

Unfortunately, Theorem 46 cannot be generalized to an arbitrary compact set $A$ of riskless alternatives without any further restrictions, as the following example shows.

Example 49 Let $A=\{(0,0),(1,1),(-1,2)\}, \bar{a}=(0,0), U_{i}=a_{i}$ for all $a \in A, i \in\{1,2\}$, $w_{1}(p)=p_{3} w_{2}(p)=\sqrt{p}, w_{2}(p)=p$ for all $p \in[0,1]$. Let $B=\left(A, \bar{a}_{3} R D U_{1}, R D U_{2}\right)$ and 
$C=\left(A, \bar{a}_{2} R D U_{1}, \widehat{R D U}_{2}\right)$. Then

$$
K S(B) \approx(0.724,1.929)
$$

but

$$
K S(C)=\left(\frac{3}{4}, \frac{9}{8}\right)
$$

Hence player 1 obtains more in the game with the more risk averse player 2 .

Nevertheless we can still partially generalize Theorem 46 .

Theorem 50 Let $B$ and $C$ be bargaining problems in that ane identical except that player 2 is move probabilistically risk averse in $C$ than in $B$. If

$$
\frac{u_{2}(B)-d_{2}(B)}{u_{1}(B)-d_{1}(B)} \leq \frac{u_{2}(C)-d_{2}(C)}{u_{1}(C)-d_{1}(C)}
$$

then $K S_{1}(C) \leq K S_{1}(B)$.

Condition (7.1) says that the line segment with $d(C)$ and $u(C)$ as endpoints is steeper (has a higher slope) than the line segment with $d(B)$ and $u(B)$ as endpoints. If $d(B)=$ $d(C)$ - which can always be arranged without loss of generality - condition (7.1) holds in particular if $U_{1}(a) \geq d_{1}(B)$ for all $a \in A$, that is, if player 1 weakly prefers all riskless alternatives to the disagreement alternative.

\subsection{An Axiomatization of the Kalai-Smorodinsky Bar- gaining Solution}

The Kalai-Smorodinsky solution for rank-dependent utility bargaining problems can be characterized by a similar set of conditions as in the expected utility case (see Kallai \& Smorodinsky, 1975). The axioms are as follows, formulated for a bargaining solution $F$.

Symmetry For every $(S, d) \in \mathbb{B}$ with (i) if $\left(s_{1}, s_{2}\right) \in S$ then also $\left(s_{2}, s_{11}\right) \in S$; (ii) $d_{1}=d_{2}$, we have $F_{1}(S, d)=F_{2}(S, d)$. 
Scale invariance For all $(S, d) \in \mathbb{S}$ and $a, b \in \mathbb{R}^{2}$ with $a>0$, we have $F(a S+b, a d+b)=$ $a F(S, d)+b$, where $a x:=\left(a_{1} x_{1}, a_{2} x_{2}\right)$ for all $x \in \mathbb{R}^{2}$ and $a S+b:=\{a s+b: s \in S\}$.

Weak Pareto optimality For every $(S, d) \in \mathbb{S}$ there is no $x \in S$ with $x>F(S, d)$.

Individual monotonicity For all $(S, d),(\bar{S}, \bar{d}) \in \mathbb{S}$ and all $i \neq j \in\{1,2\}$ with (i) $\bar{S} \subseteq S$; (ii) $\bar{d}=d$; and (iii) $u_{i}(\bar{S}, \bar{d})=u_{i}(S, d)$, we have $F_{j}(\bar{S}, \bar{d}) \leq F_{j}(S, d)$.

Observe that these conditions still make sense in the rank-dependent utility setting. In

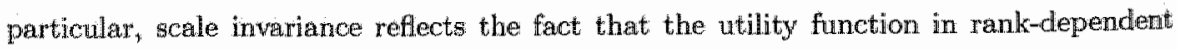
utility is unique up to a positive linear transformation. Also the following characterization is similar to the one obtained in Kalai \& Smorodinsky (1975) but in the proof (see the Appendix of the chapter) the construction of auxiliary bargaining problems needs special attention because of the different domain, in particular the presence and construction of nonconvex problems.

Theorem 51 Let $F$ be a bargaining solution. Then $F$ satisfies symmetry, scale invariance, weak Paneto optimality and individual monotonicity if, and only ij, $F=K S$.

\subsection{Conclusion}

It has been shown in this chapter that, with respect to the Kalai-Smorodinsky solution, in many situations it is on the one hand not advantageous to bargain against a more probabilistically risk averse opponent, but on the other hand also not advantageous to bargain against a less utility risk averse opponent. These results are surprising, since both probabilistic and utility risk aversion arise from the same definition of risk aversion. The intuition behind the results could be that a more utility risk averse bargainer is easier to satisfy and is therefore less demanding in a bargaining problem, whereas a more probabilistically risk averse bargainer wants a higher guarantee to obtain good outcomes and is therefore more demanding. In this chapter we varied the attitude towards risk either with respect to the weighting function or with respect to the utility function, and kept the other component fixed. It may be interesting to design an experiment to test which of the two components has more influence in bargaining problems.

The main results can be generalized to $n$-person bargaining problems with the restriction that the feasible sets are made comprehensive. It is also possible to adapt the 
results to bargaining solutions exhibiting certain monotonicity properties. For instance, the egalitarian bargaining solution defined on the set of comprehensive problens or the Kalka-Rosenthal solution (Kalai \& Rosenthal, 1978) are sucl candidates.

The extension of the Nash bargaining solution (Nash, 1950) to bargaining problems under rank-dependent utility is more problematic. In particular, feasible sets are not necessarily convex, so that the Nash bargaining solution is not uniquely defined. Nevertheless, ewen if we restrict attention to bargaining problems where the Nash bargaining solution is well defined, it does not behave regularly. Both cases can arise: a player can gain or lose with respect to the Nash bargaining solution when bargaining with a more probabilistically risk averse opponent.

Cumulative prospect theory (Tversky \& Kahneman, 1992) differs from rank-dependent utility in the sense that probabilities are weighted in a different way, depending on whether the associated riskless alternatives are preferred to a specific reference point or not. All of the above results can be generalized to cumulative prospect theory. There, a distinction between gains and losses is made, and a thind factor influences the risk attitude of a decision maker, namely loss aversion. The impact of loss aversion in bargaining problems is a subject for further research. Also Shalev (1997) considers loss aversion in bargaining but in a quite different framework.

\subsection{Appendix}

Before proving Lemmes 42 and 43 we first prove some auxiliary resultis from which these lemmas will be derived. By $\|\cdot\|$ we denote the Eucliclean norm on $\mathbb{R}^{2}$. We say that a feasible set $S$ has a hole if there is an $b \in \mathbb{R}^{2}$ and an $\varepsilon>0$ such that (i) $x \notin S$ for all $x \in \mathbb{R}^{2}$ with $\|x-h\|<\varepsilon$ and (ii) for every $x \in \mathbb{R}^{2}$ with $x \neq 0$ there is a number $\lambda_{x}>0$ with $h+\lambda_{x} x \in S$

Lemma 52 Let $B=\left(A, \bar{a}, R D U_{1}, R D U_{2}\right) \in \mathbb{B}$. Then $S(B)$ contains no holes.

Proof. Define $T=\left\{\left(R D U_{1}(P), R D U_{2}(P)\right): P \in L(A)\right\}$. By definition, $S(B)=c l\{T\}$. We say that $T$ contains an abmost-hole if there exists an $h \in \mathbb{R}^{2}$ and a $c>0$ such that (i) $x \notin S$ for all $x \in \mathbb{R}^{2}$ with $\|x-h\|<\varepsilon$ and (ii) there is a $y \in \mathbb{R}^{2}$ such that for every $x \in \mathbb{R}^{2}$ with $x \neq 0$ and $x \neq \tau y$ for every number $\tau \geq 0$ there is a number $\lambda_{s x}>0$ with 
$h+\lambda_{x} x \in S$. Since $T$ is connected and $S(B)$ is the closure of $T$, it is sufficient to show that $T$ contains no almost-holes. We first prove the following claim.

Claim. For all $s_{1} t \in T$ with either $s_{1}=t_{1}$ or $s_{2}=t_{2}$ the straight line segment between these two points is contained in $T$, i.e., $[s, t] \subseteq T$.

Proof of the Clatm: Let $s_{1} t \in T$ with $s_{2}=t_{2}$ (the case $s_{1}=t_{1}$ is analogous). Let $P=$ $\left(p^{1}, a^{1} ; \ldots ; p^{k}, a^{k}\right)$ and $Q=\left(q^{1}, b^{1} ; \ldots ; q^{n}, b^{n}\right) \in L(A)$ with $\left(R D U_{1}(P), R D U_{2}(P)\right)=s$ and $\left(R D U_{1}(Q), R D U_{2}(Q)\right)=t$. Without loss of generality assume that all probabilities in $P$ and $Q$ are positive, and let $C=\left\{a^{1}, \ldots, a^{k}, b^{1}, \ldots, b^{n}\right\}$. Let $U_{2}$ be the utility function of player 2 .

Case 1: For all $c \in C, U_{2}(c)=s_{2}$. Then all lotteries on elements of $C$ have rankdependent utility equal to $s_{2}$ for player 2 and all points in $[s, t]$ can be obtained by such lotteries. Hence, $[s, t] \subseteq T$.

Case 2: There are $c_{3} \tilde{c} \in C$ with $U_{2}(c) \neq U_{2}(\tilde{c})$. Let $\bar{c} \in \arg \max \left\{U_{2}(c): c \in C\right\}$ and $c \in \arg$ min $\left\{U_{2}(c): c \in C\right\}$. Clearly, $U_{2}(\bar{c})>s_{2}>U_{2}(c)$. Let $R=(\rho, \bar{c} ; 1-\rho, c)$ be the unique lottery with $R D U_{2}(R)=s_{2}$. Let $r=\left(R D U_{1}(R), R D U_{2}(R)\right)$, then we will show that the strajght line segment between $s$ and $r$ is in $T$. Similarly, it can be shown that the straight line segment between $r$ and $t$ is in $T$. Therefore, the straight line segment between $s$ and $t$ is contained in $T$.

Observe that for every lottery $\widetilde{P}=\left(\widetilde{p}^{1}, a^{1} ; \ldots ; \widetilde{p}^{k k}, a^{k} ; \vec{p}^{k+1}, \bar{c}^{\prime} ; \widetilde{p}^{k+2}, c\right)$ with $R D U_{2}(\tilde{P})=$ $s_{2}$, for every $i \in\{1, \ldots, k\}$ and for every $\varepsilon$ with $0 \leq \varepsilon \leq \widehat{p}^{i}$, there exist some $\lambda_{2} \pi \geq 0$ with $\lambda+\pi=\varepsilon$ such that for the lottery

$\widetilde{P^{\varepsilon}}=\left(\widetilde{p}^{1}, a^{1} ; \ldots ; \widetilde{p}^{-i-1}, a^{i-1} ; \vec{p}^{i}-\varepsilon, a^{i} ; \vec{p}^{i+1}, a^{i+1} ; \ldots ; \vec{p}^{k}, a^{k} ; \vec{p}^{k+1}+\lambda, \vec{c}_{\xi} \widetilde{p}^{k+2}+\pi, Q\right)$ we have $\operatorname{RDU}_{2}\left(\widetilde{P^{c}}\right)=s_{2}$.

Starting with $P$ we now gradually construct new lotteries with $R D U_{2}$-value equal to $s_{2}$ by shifting, for every $a^{i} \notin\{\bar{c}, c\}$, the corresponding decision weight $p^{i}$ in a continuous way simultaneously to $\bar{c}$ and $\underline{\varepsilon}$ until we reach $R_{3}$ such that the $R D V_{2}$-value stays oonstant. In this way we obtain every point on the straight line segment between $s$ and $r$ as the image of at least one of the constructed lotteries. This completes the proof of the Claim.

We now continue with the proof of the lemma. Assume for contradiction that $T$ contains an almost-hole, and let $h \in \mathbb{R}^{2}$ be as in the definition of an almost-hole. In particular, there exist $\lambda_{(1,0)}, \lambda_{(-1,0)}>0$ with $h+\lambda_{(1,0)}(1,0), h+\lambda_{(-1,0)}(-1,0) \in T$, or there exist $\lambda_{(0,1)}, \lambda_{(0,-1)}>0$ with $h+\lambda_{(0,1)}(0,1), h+\lambda_{(0,-1)}(0,-1) \in T$. Assume the former is the case, 
the latter is analogons. Observe that $\left(h+\lambda_{(1,0)}(1,0)\right)_{2}=\left(h+\lambda_{(-1,0)}(-1,0)\right)_{2}=h_{2}$. Hence the Clam implies that $\left[h-\lambda_{(-1,0)}(1,0), h+\lambda_{(1,0)}(1,0)\right] \subseteq T$. In particular, $h \in T$, which contradicts (i) in the definition of an almost-hole. "Therefore, $T$ contains no almost-holes and the proof of the lemma is complete.

The following lemma is needed to derive Lemma 43.

Lemma 53 Let $B=\left(A, \bar{a}_{2} R D U_{1}, R D U_{2}\right) \in \mathbb{B}$ with utility functions $U_{1}$ and $U_{2}$ and weighting functions $w_{1}$ and $w_{2}$ for players 1 and 2 , respectively. Let $\bar{b}^{2} \in \arg \operatorname{Max}\left\{U_{2}(a): a \in A\right\}$ and $\bar{b}^{\mathrm{i}} \in \arg \max \left\{U_{1}(a): a \in A\right\}$. Let $S=S(B)$ and $x \in P(S)$. Then

(i) if $x_{2}<U_{2}\left(\bar{b}^{2}\right)$ then for every $\varepsilon>0$ there is a $y \in S$ writh $y_{1}<x_{1}, y_{2}>x_{2,}$ and $\|x-y\|<\varepsilon$.

(ii) if $x_{1}<U_{1}\left(\bar{b}^{1}\right)$ then for every $\varepsilon>0$ there is a $y \in S$ with $y_{2}\left\langle x_{2}, y_{1}\right\rangle x_{1}$, and $\|x-y\|<\varepsilon$.

Proof: We only prove (i), the proof of (ii) is analogous. Suppose that (i) is not true. Together with $x \in P(S)$ this implies that there is a $\hat{\varepsilon}>0$ such that

$$
\operatorname{S\cap }\left\{y \in \mathbb{R}^{2}:\|y-x\|<\hat{\varepsilon}, y_{2}>x_{2}\right\}=\emptyset .
$$

Let $P \in L(A)$ be an arbitrary lottery with $R D U_{2}(P) \leq x_{2}$. Such at lottery is of the form $P=\left(p^{0}, \bar{b}^{2} ; p^{1}, a^{\sharp} ; \ldots ; p^{n}, a^{r}\right)$ with $U_{2}\left(\bar{b}^{2}\right) \geq U_{2}\left(a^{1}\right) \geq \cdots \geq U_{2}\left(a^{n}\right)$, and possibly, $p^{0}=0$.

Claim 1 There is a number $0 \leq \bar{p}<1$, independent of $P$, such that $p^{0} \leq \vec{p}$. Proof of Claim 1: Let $\underline{b}^{2} \in \arg \min \left\{U_{2}(a): a \in A\right\}$. Since $R D U_{2}(P) \leq x_{2}$ we have

$$
\begin{aligned}
x_{2} & \geq w_{2}\left(p^{0}\right) U_{2}\left(b^{2}\right)+\sum_{i=1}^{32}\left[w_{2}\left(p^{0}+\cdots+p^{i}\right)-w_{2}\left(p^{0}+\cdots+p^{i-1}\right)\right] U_{2}\left(a^{i}\right) \\
& \geq w_{2}\left(p^{0}\right) U_{2}\left(\bar{b}^{2}\right)+\sum_{i=1}^{n}\left[w_{2}\left(p^{0}+\cdots+p^{i}\right)-w_{2}\left(p^{0}+\cdots+p^{i-1}\right)\right] U_{2}\left(\underline{b}^{2}\right) \\
& =w_{2}\left(p^{0}\right)\left[U_{2}\left(\bar{b}^{2}\right)-U_{2}\left(\underline{b}^{2}\right)\right]+U_{2}\left(\underline{b}^{2}\right) .
\end{aligned}
$$

Hence, $w_{2}\left(p^{0}\right) \leq \frac{w_{2}-U_{2}\left(b^{2}\right)}{U_{2}\left(\hat{b}^{2}\right)-U_{2}\left(\underline{b}^{2}\right)}<1$, so that $p^{0} \leq w_{2}^{-1-1}\left(\frac{x_{1}-U_{2}\left(b^{2}\right)}{U_{2}\left(b^{3}\right)-U_{2}\left(\underline{b}^{2}\right)}\right):=\bar{p}<1$. This proves the claim. 
Claim 2 Let $m=\frac{1}{2}\left(x_{2}+U_{2}\left(\vec{b}^{2}\right)\right)$, and let $k \in\{1, \ldots, n\}$ such that $U_{2}\left(a^{i}\right)<m \Leftrightarrow i \geq k$. Then there is a number $0<\underline{p} \leq 1$, independent of $P$, such that $\sum_{i=k}^{n} p^{i} \geq \underline{p}$. Proof of Claim 2: Since $R D U_{2}(P) \leq x_{2}$ we have

$$
\begin{aligned}
x_{2} \geq & w_{2}\left(p^{0}\right) U_{2}\left(\vec{b}^{2}\right)+\sum_{i=1}^{n}\left[w_{2}\left(p^{0}+\cdots+p^{i}\right)-w_{2}\left(p^{0}+\cdots+p^{i-1}\right)\right] U_{2}\left(a^{i}\right) \\
\geq & w_{2}\left(p^{0}\right) U_{2}\left(\vec{b}^{2}\right)+\sum_{i=1}^{k-1}\left[w_{2}\left(p^{0}+\cdots+p^{i}\right)-w_{2}\left(p^{0}+\cdots+p^{i m-1}\right)\right] m \\
& +\sum_{i=1}^{n}\left[w_{2}\left(p^{0}+\cdots+p^{i}\right)-w_{2}\left(p^{0}+\cdots+p^{i-1}\right)\right] U_{2}\left(\underline{b}^{2}\right) \\
= & w_{2}\left(p^{0}\right) U_{2}\left(\hat{b}^{2}\right)-w_{2}\left(p^{0}\right) m+w\left(p^{0}+\cdots+p^{k-1}\right)\left[m-U_{2}\left(\underline{b}^{2}\right)\right]+U_{2}\left(\underline{b}^{2}\right) \\
\geq & w_{2}\left(p^{0}+\cdots+p^{k-1}\right)\left[m-U_{2}\left(\underline{b}^{2}\right)\right]+U_{2}\left(\underline{b}^{2}\right) .
\end{aligned}
$$

Hence, $w_{2}\left(p^{0}+\cdots+p^{k-1}\right) \leq \frac{x_{2}-U_{2}\left(B^{2}\right)}{m-U_{2}\left(\underline{b}^{2}\right)}<1$, so that $p^{0}+\cdots+p^{k-1} \leq w_{2}^{-1}\left(\frac{z_{2}-U_{2}\left(\underline{b}^{2}\right)}{m_{m}-U_{3}\left(\underline{b}^{2}\right)}\right)<1_{\text {n. }}$ and therefore $\sum_{i=k}^{n} p^{i} \geq 1-w_{2}^{-1}\left(\frac{x_{z}-U_{2}\left(b^{2}\right)}{m-U_{2}\left(\underline{d}^{2}\right)}\right):=\underline{p}>0$. This proves the claim.

Claim 3 Let $\bar{p}$ and $\underline{p}$ be es in Claims $\mathbb{d}$ and 2 , and let $0<\varepsilon \leq \min \{1-\bar{p}, \underline{p}\}$. Let $n$ and $k$ be as in Claim 2 and let $P^{\varepsilon}$ be a lottery obtained from $P$ by increasing $p^{0}$ with $\varepsilon$ and weakly decreasing, for $i=k, \ldots, n, p^{i}$ with $\varepsilon^{i} \geq 0$ such that $\sum_{i=k}^{n} \varepsilon^{i}=\varepsilon$. Then there is a number $0<r(\varepsilon) \in \mathbb{R}$, independent of $P$, such that $R D U_{2}\left(P^{\varepsilon}\right) \geq R D U_{2}(P)+r(\varepsilon)$.

Proof of Claim 3: Define $\underline{w}(\varepsilon):=\min \left\{w_{2}(q+\varepsilon)-w_{2}(q): 0 \leq q \leq 1-\varepsilon\right\}$. Observe that $\underline{w}(\varepsilon)>0$. By rearranging the expression in the definition of RDU and setting $a^{0}:=\bar{b}^{2}$, we have:

$$
\begin{aligned}
R D U_{2}\left(P^{e}\right)= & \sum_{i=0}^{k-1} w_{2}\left(\left(p^{0}+\varepsilon\right)+p^{1}+\cdots+p^{i}\right)\left[U_{2}\left(a^{i}\right)-U_{2}\left(a^{i+1}\right)\right] \\
& +\sum_{i=k}^{n-1} w_{2}\left(\left(p^{0}+\varepsilon\right)+p^{\mathbb{1}}+\cdots+p^{b-1}\right. \\
& \left.+\left(p^{k}-\varepsilon^{k}\right)+\cdots+\left(p^{i}-\varepsilon^{i}\right)\right)\left[U_{2}\left(a^{i}\right)-U_{2}\left(a^{i+1}\right)\right]+U_{2}\left(a^{n}\right) \\
\geq & \sum_{i=0}^{k-1} w_{2}\left(\left(p^{0}+\varepsilon\right)+p^{\mathbb{1}}+\cdots+p^{i}\right)\left[U_{2}\left(a^{i}\right)-U_{2}\left(a^{i+1}\right)\right] \\
& +\sum_{i=k}^{n-1} w_{2}\left(p^{0}+p^{1}+\cdots+p^{i}\right)\left[U_{2}\left(a^{i}\right)-U_{2}\left(a^{i+1}\right)\right]+U_{2}\left(a^{n}\right)
\end{aligned}
$$




$$
\begin{aligned}
& \geq \sum_{i=0}^{k-1}\left[w_{2}\left(p^{0}+p^{1}+\cdots+p^{i}\right)+\underline{w}(\varepsilon)\right]\left[U_{2}\left(a_{i}^{i}\right)-U_{2}\left(a^{i+1}\right)\right] \\
& \quad+\sum_{i=k}^{n-1} w_{2}\left(p^{0}+p^{1}+\cdots+p^{i}\right)\left[U_{2}\left(a^{i}\right)-U_{2}\left(a^{i+1}\right)\right]+U_{2}\left(a^{n}\right) \\
& =\operatorname{RDU}_{2}(P)+\underline{u}(\varepsilon)\left[U_{2}\left(a^{0}\right)-U_{2}\left(a^{k}\right)\right] \\
& \geq \operatorname{RDU}_{2}(P)+\underline{w}(\varepsilon)\left[U_{2}\left(b^{2}\right)-m .\right.
\end{aligned}
$$

The claim follows by defining $r(\varepsilon):=\underline{w}\left(\varepsilon_{d}^{y} \mid U_{2}\left(\bar{b}^{2}\right)-m\right]$.

For the next claim, let $\underline{b}^{2} \in \arg \min \left\{U_{1}(a): a \in A\right\}$.

Chaim 4 Let $\eta>0$ and let $Q=\left(q^{1}, a^{k} \ldots ; q^{n}, a^{n}\right)$ and $Q^{n}=\left(q^{1}, a^{1} ; \ldots ; \hat{q}^{n}, a^{n}\right)$ be arbitrary lotteries such that $Q^{\eta}$ arises from $Q$ by shifting arourd probability such that $\sum_{i=\|}^{n}\left|q^{i}-\vec{q}^{i}\right| \leq \eta$. Then there are positive numbers $M_{1}(\eta)$ and $M_{2}(\eta)$, independent of $Q$, such that $\left\|R D U_{2}(Q)-R D U_{1}\left(Q^{\prime}\right)\right\| \leq M_{1}(\eta)$ and $\left|R D U_{2}(Q)-R D U_{2}\left(Q^{\prime \prime}\right)\right| \leq M_{2}(\eta)$, and, moreover, $M_{1}(\eta), M_{2}(\eta) \rightarrow 0$ as $\eta \rightarrow 0$.

Proof of Claim 4: We only prove the first inequality, the proof of the second one is analogous. Without loss of generality assume $U_{1}\left(a^{1}\right) \geq \cdots \geq U\left(a^{n}\right)$. Then

$$
\begin{aligned}
& \left|R D U_{1}(Q)-R D U_{1}\left(Q^{n}\right)\right|=\mid \sum_{i=1}^{n_{n}-1} w_{1}\left(q^{1}+\cdots+q^{i}\right)\left[U_{1}\left(a^{i}\right)-U_{1}\left(a^{i+1}\right) \mid+U_{1}\left(a^{n}\right)\right. \\
& \left.-\sum_{i=1}^{n-1} w_{1}\left(a^{-1}+\cdots+\tilde{q}^{i}\right)\left[U_{1}\left(a^{i}\right)-U_{1}\left(a^{i+1}\right)\right]-U_{1}\left(a^{n}\right)\right] \\
& =1 \sum_{i=1}^{n-1}\left[w_{1}\left(q^{1}+\cdots+q^{i}\right)-w_{1}\left(\tilde{q}^{1}+\cdots+\tilde{q}^{i}\right)\right] . \\
& \left\{U_{1}\left(a^{i}\right)-U_{1}\left(a^{i+1}\right)\right] \\
& \leq \sum_{i=1}^{n+1} \max \left[\left|w_{1}(q+\eta)-w(q)\right|: 0 \leq q \leq 1-\eta_{i}\right\} \\
& \left.\mid U_{1}\left(a^{i}\right)-U_{1}\left(a^{i+1}\right)\right\rfloor \\
& \leq M_{1}(\eta)
\end{aligned}
$$

with $M_{1}(\eta):=\max \left\{\mid w_{1}\left(q+q_{1}\right)-u(q): 0 \leq q \leq 1-\eta\right\}\left[U_{1}\left(b^{-1}\right)-U_{1}\left(b^{1}\right)\right\}>0$. Since $M_{1}(\eta) \rightarrow 0$ as $\eta \rightarrow 0$ the proof of Claim 4 is complete. 
We will now complete the proof of the lemma. Take a sequence of lotteries $P_{1}, P_{2}, \ldots$ with $\left(R D U_{1}\left(P_{\ell}\right), R D U_{2}\left(P_{\ell}\right)\right) \rightarrow x$ as $\ell \rightarrow \infty$. Assume without loss of generality that $\left\|\left(R D U_{1}\left(P_{\ell}\right), R D U_{2}\left(P_{\ell}\right)\right)-x\right\|<\frac{\varepsilon^{2}}{4}$ for every $\ell$. By $(7.2), R D U_{2}\left(P_{\ell}\right) \leq x_{2}$ for every $\ell$. Take $\eta>0$ so small that $M_{1}(\eta), M_{2}(\eta)<\frac{z}{4}$ (with $M_{1}(\eta), M_{2}(\eta)$ as in Claim 4.). Finally, take $\varepsilon>0$ with $2 \varepsilon<\eta$ and $0<\varepsilon \leq m i n\{1-\bar{p}, \underline{p}\}$ (as in Claim 3). Then Claim 3 implies that for an $\ell^{\prime}$ sufficiently large we have $R D U_{2}\left(P_{\ell^{\prime}}^{\varepsilon}\right) \geq R D U_{2}\left(P_{\ell^{\prime}}\right)+r(\varepsilon)>x_{2}$, with $P_{\ell^{\prime}}^{\varepsilon}$ and $r(\varepsilon)$ as in Claim 3. Since $2 \varepsilon<n$ it follows by Claim 4 that $\left|R D U_{\mathbb{1}}\left(P_{z^{\prime}}\right)-R D U_{1}\left(P_{\varepsilon^{\prime}}^{\varepsilon^{\prime}}\right)\right| \leq$ $M_{\mathbb{1}}(\eta)$ and $\left|R D U_{2}\left(P_{g^{\prime}}\right)-R D U_{2}\left(P_{\ell^{\prime}}^{\varepsilon^{\prime}}\right)\right| \leq M_{2}(\eta)$. Hence, $\left|R D U_{1}\left(P_{q^{\prime}}\right)-R D U_{1}\left(P_{\ell^{\prime}}^{E^{\prime}}\right)\right| \leq \frac{1}{4}$

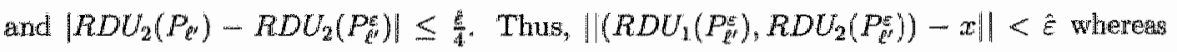
$R D U_{2}\left(P_{k^{\prime}}^{q}\right)>x_{2}$, contradicting $(7,2)$. This completes the proof of lemma.

Proof of Lemma 43: Let $\bar{x}$ be the point of $S$ with maximal first coordinate among the points of $S$ with maximal second coordinate. Similarly, let $x$ be the point of $S$ with maximal second coordinate among the points of $S$ with maximal first coordinate. Clearly, $\bar{x}$ and $\underline{x}$ are Pareto optimal points, and for every point $x \in P(S)$ we have $\bar{x}_{1} \leq x_{1} \leq \underline{x}_{1}$.

To prove the lemma it is sufficient to prowe that for every $\alpha \in \mathbb{R}$ with $\widetilde{x}_{1} \leq \alpha \leq \underline{x}_{1}$ there is a number $f(\alpha) \in \mathbb{R}$ with $(\alpha, f(\alpha)) \in P(S)$ : such a number must then be mique and the implied function $f$ is strictly decreasing and, by Lemma 53 , also continuous. Hence, $f$ satisfies the requirements of the lemma.

For $\alpha=\bar{x}_{1}$ take $f(\alpha)=\bar{x}_{2}$ and for $\alpha=\underline{x}_{1}$ take $f(\alpha)=\underline{x}_{2}$. For $\alpha \in \mathbb{R}$ with $\bar{x}_{1}<\alpha<\underline{x}_{1}$ define $f(\alpha):=\max \{\beta:(\alpha, \beta) \in S\}$. Suppose $(\alpha, f(\alpha)) \notin P(S)$. Then let $x$ be the point with maximal first coordinate among all points of $D:=\{y \in S: y \geq(\alpha, f(\alpha))\}$ with maximal second coordinate. Clearly, $x \in P(S), x_{1}>\alpha$, and $x_{2}<\bar{x}_{2}$. Then Lemma 53.(i) implies that there is a point $y \in D$ withl $y_{2}>x_{2}$, a contradiction to the definition of $x$. This completes the proof of the lemma.

For $(S, d) \in \mathbb{S}$ we define

$P^{+1}(S)=\left\{s \in S\right.$ : there exists no $t \in S$ with $t_{1} \geq s_{1}, t_{2} \geq s_{2}$ and $\left.t \neq s\right\}$, $P^{+-}-(S)=\left\{s \in S:\right.$ there exists no $t \in S$ with $t_{1} \geq s_{1}, t_{2} \leq s_{2}$ and $\left.t \neq s\right\}$. $P^{-1}(S)=\left\{s \in S:\right.$ there exists no $t \in S$ with $t_{1} \leq s_{1}, t_{2} \geq s_{2}$ and $\left.t \neq s\right\}$, and $P^{\cdots-1}(S)=\left\{s \in S:\right.$ there exists no $t \in S$ with $t_{1} \leq s_{1}, t_{2} \leq s_{2}$ and $\left.t \neq s\right\}$.

Observe that $P^{++}(S)=P(S)$.

The following lemma is a generalization of Lemma 43. The proof is analogous to the proof of Lemma 43. It is therefore omitted. The lemma is used in the proof of Lemma 42. 
Lemma 54 Let $(S, d) \in \mathbb{S}$. Ther there are closed intervals $I^{+4}, I^{+-}, I^{-+}$, and $I^{-\infty} \subseteq \mathbb{R}$, contwinons strictly decreasing functions $f^{++}: I^{++} \rightarrow \mathbb{R}^{+-}: f^{-m} \rightarrow \mathbb{R}$ and continuous stretly ancreasing functions $f^{+-}: I^{+-} \rightarrow \mathbb{R}, f^{++}: I^{-+} \rightarrow \mathbb{R}$ such that

$$
\begin{aligned}
& P^{++}(S)=\left\{\left(t, f^{++}(t)\right): t \in I^{++}\right\}, \\
& P^{+-}(S)=\left\{\left(t, f^{+-}(t)\right): t \in I^{+-}\right\}, \\
& P^{-+}(S)=\left\{\left(t, f^{-+}(t)\right): t \in I^{-+}\right\}, \\
& P^{--}(S)=\left\{\left(t, f^{--}(t)\right): t \in I^{--}\right\} .
\end{aligned}
$$

Proof of Lemma 42: We first observe that the points of $S$ with maximal second coordinates form a straight line segment, possibly consisting of only one point: all these points can be obtained by lotteries between riskless alternatives with mimimal and maximal utility for player 1 among all the riskless altematives that have maximal utility for player 2. The same is true for all points with minimal second coordinates, or maximal or minimal first coondimates.

Let $s, t \in S$ be points that have one coordinate in common. Without loss of generality assume $s_{1}<t_{1}$ and $s_{2}=t_{2}$. If $s_{2}$ is maximal or minimal in $S$ then $[s, t] \subseteq S$ follows from the observations in preceding paragraph. Otherwise, any point $\left.h \in \| s_{s} t\right]$ strictly between $s$ and $t$ satisfies condition (ii) in the definition of a hole: this follows from the observations in the preceding paragraph and from Lemma 54. Suppose that there would be such an $h$ with $h \notin S$. Since $S$ is closed there nust be an $\varepsilon$-neighborhood of h that is not in $S$. Hence, $h$ satisfies also condition (i) in the definition of a hole, and thus $S$ has a hole. This contradicts Lemma 52 and completes the proof.

Proof of $\mathbb{L}$ emma 44: Let $I=[\alpha, \beta]$. If $\alpha \neq \beta$, define the continuous strictly increasing function $w:[0,1] \rightarrow[0,1]$ by

$$
w\left(\frac{\beta-t}{\beta-\alpha}\right)=\frac{f(t)-f(\beta)}{f(\alpha)-f(\beta)}
$$

for every $t \in I$. In particular, $w(0)=0$ and $w(1)=1$, so that $w$ satisfies all the requirements of a weighting function. If $\alpha=\beta$, then for $\bar{\alpha}$ take any point in $\mathbb{R}^{2}$ with $\bar{a}<(\alpha, f(\alpha))$, and define $A=\left\{\bar{a}_{,}\left(\alpha, \bar{a}_{2}\right),(\alpha, f(\alpha)),\left(\bar{a}_{1,} f(\alpha)\right)\right\}$. If $\alpha \neq \beta$ then take $\bar{\alpha}=(\alpha, f(\beta))$, and define $A=\{\bar{a},(\beta, f(\beta)),(\alpha, f(\alpha))\}$. Define $U_{i}(\alpha)=a_{i}$ for all $a \in A$ and $i \in\{1,2\}$, 
$w_{1}(p)=p$ and $w_{2}(p)=w(p)$ for all $p \in[0,1]$. Let $B=\left(A, \bar{a}_{1}, R D U_{1}, R D U_{2}\right)$. By construction, $\{(t, f(t)): t \in I\}=\left\{\left(R D U_{1}(P), R D U_{2}(P)\right): P=((\alpha, f(\alpha)), p,(\beta, f(\beta)), 1-\right.$ $p)$ for some $p \in[0,1]\}$. This is the set $P(B)$, and by Lemma $42, S(B)=\left\{x \in \mathbb{R}^{2}: \bar{a} \leq\right.$ $x \leq(t, f(t))$ for some $t \in I)$.

Proof of Theorem 45: Let $B=\left(A, \bar{\alpha}_{1} R D U_{1}, R D U_{2}\right)_{\text {s }}$ $C=\left(A, \bar{a}_{n} R D U_{1}, \widehat{R D U_{2}}\right) \in \mathbb{B}^{*}$ such that $R D U_{2}$ is associated with $U_{2}$ and $\omega_{2}$ and $\widehat{R D U_{3}}$ with $\widetilde{U}_{2}=k \circ U_{2}$ and $w_{2}$ for a concave strictly increasing tramaformation $k: \mathbb{R} \rightarrow \mathbb{R}$. It can be assumed that $\widetilde{U_{2}}$ is scaled such that $d(B)=d(C)$ and $u(B)=u(C)$, since the Kala:Smorodinsky solution is scale invariart (see Theorem 51). This implies that $\widetilde{U}_{2}(a) \geq U_{2}(a)$ for all $a \in A$ and therefore,

$$
\widehat{R D U_{12}}(P) \geq R D U_{2}(P)
$$

for all $P \in L(A)$. Consider $K S(B)$. Because $d(B)=d(C)$ and $u(B)=u(C)$, we either have $K S(B) \leq K S(C)$ or $K S(B)>K S(C)$. Weak Pareto optimality of the Kalai-Smorodinsky solution (see Theorem 51) and (7.3) imply $K S(B) \leq K S(C)$, hence $K S_{1}(B) \leq K S_{1}(C)$.

Proof of Theorem 46: Let $B=\left(A, \bar{\alpha}_{1} R D U_{1}, R D U_{2}\right)$, $C=\left(A, \bar{a}_{1} R D U_{1}, \widehat{R D U_{2}}\right) \in \mathbb{B}^{+}$such that $R D U_{2}$ is associated with $U_{2}$ and $w_{2}$ and $\widehat{R D U}_{2}$ with $U_{2}$ and $\widetilde{w}_{2}$ where $w_{2}(p) \geq \bar{w}_{2}(p)$ for all $p \in[0,1]$. (Hence, without loss of generality we have scaled the utility function of player 2 in $C$ such that it is equal to $U_{2}$, cf. Lemma 41.) Then by Lemma 41 , part 2 ,

$$
\operatorname{RDU}_{2}(P) \geq \widehat{R D U}_{2}(P)
$$

for all $P \in L(A)$. Consider $K S(C)$. Because $d(B)=d(C)$ and $u(B)=u(C)$, either $K S(C) \leq K S(B)$ or $K S(C)>K S(B)$. Weak Pareto optimality of the Kalai-Smorodinsky solution (see Theorem 51 ) and $(7.4)$ imply $K S(C) \leq K S(B)$, hence $K S_{1}(B) \geq K S_{1}(C)$.

Proof of Theorem 50: Let $B=\left(A, \bar{a}_{3} R D U_{1}, R D U_{2}\right)$, $C=\left(A, \vec{a}, R D U_{1}, \widehat{R D U_{2}}\right) \in \mathbb{B}$ such that $R D U_{2}$ iss associated with $U_{2}$ and $w_{2}$ and $\widehat{R D U}_{2}$ with $U_{2}$ and $\tilde{w}_{2}$ where $w_{2}(p) \geq \tilde{w}_{2}(p)$ for all $p \in[0,1]$ - this is similar as in the proof of Theorem $46-$ and $\frac{u_{7}(B)-d_{3}(B)}{u_{1}(B)-d_{1}(B)} \leq \frac{w_{2}(C)-d_{2}(O)}{u_{4}(C)-d_{1}(C)}$. Because the Kalai-Smorodinslky solution is 


\section{APPENDIX}

scale invariant (see Theorem 51 ) we can assume without loss of generality that $d(B)=$ $d(C)=(0,0)$.

Define $H(C)$ to be the maximal point of $S(C)$ on the line segment with $d(B)$ and $u(S(B), d(B))$ as endpoints. As in the proof of Theorem 46 , it can be shown that

$$
H(C) \leq K S(B)
$$

From Lemmina 43 it follows that $H(C)$ is Pareto optimal. Suppose that $K S_{1}(C)>H_{1}(C)$. Then, $K S_{2}(C)=\frac{w_{2}(C)}{u_{1}(C)} K S_{1}(C) \geq \frac{u_{2}(B)}{u_{1}(B)} K S_{1}(C)>\frac{u_{2}(S)}{u_{1}(B)} H_{1}(C)=H_{2}(C)$. This contradicts the Pareto optimality of $H(C)$. It follows that

$$
K S_{1}(C) \leq H_{\mathbb{1}}(C)
$$

From $(7.5)$ and $(7.6)$,

$$
K S_{1}(C) \leq K S_{1}(B)
$$

which was to be proved.

Proof of Theorem 51: Symmetry, scale invariance and individual monotonicity of the Kalai-Smorodinsky solution are immediate. (Weak) Pareto optimality follows from Lemma 43.

Let $F$ be a bargaining solution which satisfies symmetry, scale invariance, weak Pareto optimality and individual monotonicity. We show that, $F=K S$. Our proof deviates from the proof in Kalai \& Smorodinsky (1975) because also certain nonconvex feasible sets are perrouitted.

Let $(S, d) \in \mathbb{S}$. Define $T=\{a s+b: s \in S\}$ with $a=\left(\frac{l}{u_{1}(S, d)-d_{1}}, \frac{d}{\left.u_{1}\left(S_{n} d\right\}\right)-d_{2}}\right)$ and $b=-a d$. Then $(T, 0) \in \mathbb{S}$, (where we write 0 instead of $(0,0))$ and scale invariance implies

$$
\begin{aligned}
K S(T, 0) & =a K S(S, d)+b \text { and } \\
F(T, 0) & =a F(S, d)+b .
\end{aligned}
$$

So it is sufficient to prove that $K S(T, 0)=F(T, 0)$. 
Note that $u(T, 0)=(1,1)$ and, therefore,

$$
K S_{1}(T, 0)=K S_{2}(T, 0)
$$

Let $C=\left(A, \bar{a}_{1} R D U_{1}, R D U_{2}\right) \in \mathbb{R}$ such that $T=S(C)$ and $0=d(C)$. Let $U_{1}$ and $U_{2}$ be the associated utility functions.

First consider the case that $u(T, 0)=(1,1) \in T$, hence $K S(T, 0)=(1,1)$. Since, by Lemmat $43, P(T)=\{(1,1)\}$, there must be an $a \in A$ with $(1,1)=\left(R D U_{1}(a), R D U_{2}(a)\right)$. Define $C^{\prime}=\left(\{\bar{a}, a\}, \bar{a}_{1} R D U_{1}, R D U_{2}\right) \in \mathbb{B}$ where $R D U_{1}$ and $R D U_{2}$ are the restrictions of the rank-dependent utility functionals in $C$. With $L:=S\left(C^{\prime}\right)$, we have $F(L, 0)=(1,1)$ by weak Pareto optimality of $F$. By individual monotonicity of $F$ applied to $L \subseteq T$ we have $F(T, 0) \geq F(L, 0)=(1,1)$, hence $F(T, 0)=(1,1)=K S(T, 0)$. This completes the proof of the theorem for this case.

Second and last, consider the case that $u(T, 0)=(1,1) \notin T$, hence $K S(T, 0)<(\mathbb{1}, 1)$. By Lemma 43 there is a function $f$, the graph of which coincides with $P(T)$. Define $y:=\left(\min \left\{x_{1}: x \in T\right\}, \min \left\{x_{2}: x \in T\right\}\right)$. Let $B=\left(\bar{A}_{1}(0,0), \overline{R D U}_{1}, \overline{R D U}_{2}\right)$, where $\bar{A}=\left\{\left(U_{1}(a), U_{2}(a)\right): a \in A\right\} \cup\left\{y,\left(y_{1}, \max \left\{x_{2}: x \in T\right\}\right),\left(\max \left\{x_{1}: x \in T\right\}, y_{2}\right)\right\}$, with the utility functions $\bar{U}_{i}$ defined by $\bar{U}_{i}(x)=x_{i}$ for every $x \in \bar{A}$, and with weighting functions $\bar{w}_{i}$ equal to those in $C$. Then $d(B)=0, P(B)=P(T)$, and

$$
S(B)=\left\{x \in \mathbb{R}^{2}: y \leq x \leq x^{\prime} \text { for some } x^{\prime} \in P(T)\right\}
$$

Denote $\tilde{T}=S(B)$. Then $u(\widetilde{T}, 0)=(1,1)$ and $T \subseteq \widetilde{T}$, so by individual monotonicity of $F, F(\widetilde{T}, 0) \geq F(T, 0)$. Since $K S(\widetilde{T}, 0)=K S(T, 0)$, it is sufficient to show that $F(\widetilde{T}, 0)=$ $K S(\widetilde{T}, 0)$ : then $K S(T, 0) \geq F(T, 0)$ which implies $K S(T, 0)=F(T, 0)$ since $K S(T, 0)<$ $(1,1)$.

Let $z:=K S(\tilde{T}, 0)\left(\right.$ so $\left.z_{1}=z_{2}\right)$ and let $f:[0,1] \rightarrow[0,1]$ be a continuous strictly decreasing function with $(t, f(t)) \in \widetilde{T}, f\left(z_{1}\right)=z_{2}$, and $f(t)=f^{-1}(t)$ for every $t \in[0,1]$. Then $f(0)=1, f(1)=0$, and by Lemma 44 and its proof there is a $(V, 0) \in \mathbb{S}$ with $P(V)$ equal to the graph of $f$ and $V=\left\{x \in \mathbb{R}^{2}: 0 \leq x \leq x^{\prime}\right.$ for some $\left.x^{\prime} \in P(V)\right\}$. Because $V$ is symmetric by construction, symmetry and weak Pareto optimality of $F$ imply $F(V, 0)=z$. Since $u(V, 0)=(1,1)$ and $V \subseteq \widetilde{T}$, individual monotonicity of $F$ implies $F(\widetilde{T}, 0) \geq F(V, 0)=z$, hence $F(\tilde{T}, 0)=z=K S(\widetilde{T}, 0)$ since $z \in P(\widetilde{T})$. This completes the proof of the second case, and hence of the theorem. 


\section{Chapter 8}

\section{Loss Aversion in Bargaining}

\section{Problems}

This chapter examines the influence of loss aversion in bargaining problems under prospect. theory. For the Kalai and Smorodinsky and the Kalai and Rosenthal bargaining solutions, it is shown that it is aswally better for a player if the opponent is less loss averse. The Nash bargaining solution does not behave wery regular in this respect."

\subsection{Introduction}

A bargaining problem is described by a set of potential alternatives for the bargainers, including a so-called disagreement alternative. The bargainers try to reach an agreement on one of the alternatives or on a lottery between the alternatives. If they fail, the disagreement alternatives results. A bargaining solution suggests an agreement lottery for each possible bargaining problem.

An interesting question, first studied by Kannai (1977) and Kihlstrom, Roth, \& Schmeidier (1981) for the case that both barganers maximize expected utility, concerns the effect of risk aversion on the outcomes predicted by bargaining solutions. Their answers to this question confirm a plausible intuition: in most cases it is advantageous to play against a more risk averse bargainer. These results are drwen by the fact that a more risk averse bargainer has a more concave ubility function. Therefore, utility at low levels increases at a

\footnotetext{
${ }^{1}$ The resnits in this chapter were furs formulated in (Kobberling \& Peters, 2001).
} 
faster rate and satisfaction may appear faster. Because most bargaining solutions are sensitive to this, a more risk averse bargainer may be easier to satisfy, which is advantageous for the opponent (cf. Peters, 1992).

Kobberling \& Peters (2002, see Chapter 7) considered bargaining problems where the bargainers' preferences can be represented by rank-dependent utility (Quiggin, 1982). Rank-dependent utility is a generalization of expected utility and allows for specific transformations of probabilities. In contrast to expected utility, not only the utility function but also a probability weighting function determines the risk attitude of a decision maker. Two forms of risk aversion can be distinguished: utility risk aversion and probabilistic risk aversion. Surprisingly, for some bargaining solutions (like the solution of Kalai \& Smorodinsky, 1975) it turns out that these two forms of risk aversion usually have opposite effects: It is advantageous to play against a more utility risk averse player, but disadvantageous to play against a more probabilistically risk averse bargainer. These results are, however, not counterintuitive. The marginal utility of a more utility risk averse opponent diminishes faster, and satisfaction may appear faster. On the other hand, a more probabilistically risk averse opponent underweighs probabilities for high outcomes, and might, therefore, insist on larger probabilities for good outcomes.

Prospect theory (Kahneman \& Tversky, 1979; Tversky \& Kahneman, 1992) is a generalization of rank-dependent utility. In adidition to the transformation of probabilities as under rank-dependent utility, outcomes are valued as gains and losses relative to some reference point. Loss aversion is a third component of observed risk aversion, and can be modeled in prospect theory because of the existence of a reference point. It refers to the prevalent obserwed behavior that usually more attention is paid to potential losses than to potential gains (Kahneman, Knetsch \& Thaler, 1991). Köbberling \& Wakker (2002c, see Chapter 5) discussed extensively the empirical evidence and relevance of loss aversion, and introduced a quantitative index of loss aversion.

In bargaining problems it is a plausible assumption that the disagreement alternative serves as a reference point for the players. Therefore the distinction between gains and losses is a natural idea in bargaining game theory and loss aversion can be expected to play an importent role. This chapter considers bargaining problems, where the preferences of the players are modeled by prospect theory. Three components of risk aversion have an impact on the bargaining solution: utility risk aversion, probabilistic risk aversion and loss aversion. Chapter 7 discussed the first two under rank-dependent utility. Loss aversion, 
however, is a new component.

It turns out that in many bargaining situations the more loss averse the opponent is, the worse it is for a player. This holds, in particular, for the Kalai-Rosenthal solution (Kalai \& Rosenthal, 1978) and for the Kalai-Smorodinsky solution (Kalai \& Smorodinsky, 1975). The intuition behind this observation is that a more loss averse bargainer may ask for higher compensation for potential losses. Furthermore, this chapter discusses strategic implications for the opponent, under the assumption that it is possible to be dishonest about the true degree of loss aversion.

Shalev (1997) also studied the effect of loss aversion on bargaining, but in a different framework. He assumed that the mage of the set of potential alternatives in utility space is a convex set, whereas in the present paper it can be any compact set. Also, he does not allow for lotteries over the set of alternatives. In the present chapter the reference point is exogenously given by the disagreement outcome. Shalev assumed that the reference point can be any alternative. He defined so-called self-supporting and stable reference points and examined the influence of loss aversion on these points.

\subsection{Preliminaries}

\subsubsection{Prospect Theory and Loss Aversion}

Thronghout, the set $A$ of alternatives is a nonempty compact topological space. A lottery is a probability measure with finite support on $A$, typically denoted by $P=$ $\left(p^{1}, a^{1} ; \ldots ; p^{n}, a^{n}\right)$ where $a^{1}, \ldots, a^{n} \in A, p^{i} \geq 0$ for all $i$, and $\sum_{i=1}^{n} p^{i}=1$. The set of all lotteries is $L(A)$. Each alternative $a \in A$ is identified with the riskless lottery $(1, a) \in L(A)$.

The preferences of an agent over lotteries are denoted by $\succcurlyeq$, with indifference denoted by $\sim$, and strict preference denoted by $\succ$. A designated alternative $r \in A$ serves as the reference point for the agent, with respect to which all other alternatives are either gatns, if $a \succ r$, or losses, if $a \prec r$. Each lottery $P$ is associated with a rank-ordering permutation $\rho$ that orders the alternatives in $P$ from best to worst, such that $\alpha^{\rho(1)} \succcurlyeq \cdots \geqslant a^{\rho(k)} \geqslant r$; $a^{\rho(k+2+1)} \geqslant \ldots \neq a^{\rho(n)}$ for some $0 \leq k \leq n$.

The new version of prospect theory (Tversky \& Kahneman, 1992) holds if an cevaluation function $\mathrm{CPT}$ represents preferences (i.e., $P \geqslant Q \Leftrightarrow C P T(P) \geq C P T(Q)$ for all lotteries $P, Q$ ), where CPT is defined as follows. 
(1) There existes a wtilty function $U: A \rightarrow \mathbb{R}$ with $U(r)=0$.

(ii) There exist weighting functions $w^{4}, w^{-\prime}:[0,1] \rightarrow[0,1]$, which are continuous and strictly increasing with $w^{+}(0)=w^{-}(0)=0$ and $w^{+}(1)=w^{-}(1)=1$.

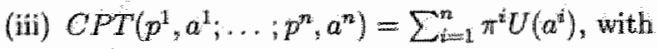

$\pi^{p(i)}=w^{+(}\left(p^{p(1)}+\cdots+p^{p(i)}\right)-w^{n}\left(p^{\rho(1)}+\cdots+p^{p(i-1)}\right)$ for $i \leq k$ and

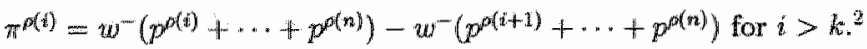

Prospect theory assigns to each lottery some weighted average utility, where the weights are subjective and depend on the probabilities, the rank-ordering, and the reference point.

Loss aversion is an important empirical phenomenon. It refers to the prevalent observed behavior that the negative utility ausigned to losses is usually bigger than the utility assigned to comparable gains. Because of the existence of a reference point, prospect theory is a decision theoretic model that can incorporate loss aversion.

Kobberling \& Wakker (2002c, see Chapter 5) discussed the idea of a basic utility function $\mathbf{u}$ reflecting the intrinsic value of outcomes for the agent. The observable utility $U$ is a composition of a loss aversion index $\lambda>0$, and the basic utilty u. Formally,

$$
U(a)= \begin{cases}\mathbf{u}(a) & \text { if } a \succcurlyeq r \\ \lambda_{\mathbf{u}}(a) & \text { if } a \prec r\end{cases}
$$

The loss aversion index captures the exchange rate between gain- and loss- utility units.

To differentiate between two CPT evaluation functions with different loss aversion indices $\lambda$ and $\mu$, but the same weighting functions $w^{+}, w^{-}$and the same basic utility $u$, the notations $C P T^{\wedge}$ and $C P T^{\mu}$ are used. The following observation is straightforward.

Observation 55 Let $\lambda>\mu$. Then $C P T^{\lambda}(P) \leq C P T^{\mu}(P)$ for every $P \in L(A)$.

\subsubsection{Bargaining Problems and Solutions}

As before, a nonempty compact topological space $A$ is the set of riskless altematiwes. Two bargainers try to reach an agreement in $L(A)$. A designated element $\bar{a} \in A$ is the disagnement altermative. The disagreement alternative serves as the reference point fot"

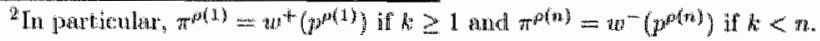


the two bargainers, which are assumed to have preferences according to prospect theory exaluation functions $C P T_{1}$ and $C P T_{2}: L(A) \rightarrow \mathbb{R}^{2}$. In particular, we have $C P T_{1}(\bar{a})=$ $C P T_{2}(\bar{a})=0$. If the bargainers fail to come to an agreement, $\bar{a}$ results. We assume throughout that there is a lottery $P \in L(A)$ with $C P T_{1}(P)>0$ and $C P T_{2}(P)>0$. The quadruple $\left(A, \bar{a}, C P T_{1}, C P T_{2}\right)$ is a (two-person) bangaining problem. $\mathbb{B}$ is the set of all possible bargaining problems.

For a bargaining problem $B=\left(A, \bar{a}, C P T_{1}, C P T_{2}\right)$, define

$$
S(B):=\operatorname{cl}\left\{\left(C P T_{1}(P), C P T_{2}(P)\right): P \in L(A)\right\}
$$

where "cl" denotes the (topological) closure. The set $S(B)$ is called the feasible set of $B$. It is a compact (not necessarily convex) subset of $R^{2}$. In contrast to the case of bargaining problems under expected utility, examples (with infinite A) can be constructed where the set $\left\{\left(C P T_{1}(P), C P T_{2}(P)\right): P \in L(A)\right\}$ is not closed.

We define

$$
\mathbb{S}=\{S: S=S(B) \text { for some } B \in \mathbb{B}\}
$$

A bargaining solution is a map $F: S \rightarrow \mathbb{R}^{2}$ that assigns to each $S \in \mathbb{S}$ an element of $S$. In the rest of the chapter, we also write $F(B)$ instead of $F(S(B))$.

For $S \in \mathbb{S}$ the Paneto optimal set $P(S)$ is defined by

$$
P(S)=\{s \in S: \text { there exists no } t \in S \text { with } t \geq s \text { and } t \neq s\} \text {. }
$$

As under rank-dependent utility (see Chapter 7) it can be shown that $P(S)$ is a connected subset of $S$

\subsubsection{The Kalai-Smorodinsky Bargaining Solution}

For $S \in \mathbb{S}$, the utopia point is defined by:

$$
u(S)=\left(\max \left\{s_{1}: s \in S, s \geq(0,0)\right\}, \max \left\{s_{2}: s \in S, s \geq(0,0)\right\}\right)
$$

For $B \in \mathbb{B}$ we also write $u(B)$ instead of $u(S(B))$. The Kalai-Smorodinsky solution $K S$ (Kalai \& Smorodinsky, 1975) assigns to each $S \in \mathbb{S}$ the maximal point of $S$ on the line 
segment with $(0,0)$ and $u(S)$ as endpoints. It is well defined because $S$ is compact. Clearly, $K S(S) \in P(S)$ for every $S \in \mathbb{S}$.

The definition of this solution concept is an adaptation of the definition for expected utility to prospect theory. Kalail \& Smorodinsky (1975) justified and characterized the solution concept by a set of axioms. In (Kobberling \& Peters, 2002, see Chapter 7) it was. shown that this axiomatization can be extended to rank-dependent utility. Similarly, it can be extended to prospect theory, but we will not elaborate on this.

\subsubsection{The Kalai-Rosenthal Bargaining Solution}

For $S \in \mathbb{S}$, the global utopia point is defined by:

$$
g(S)=\left(\max \left\{s_{1}: s \in S\right\}_{3} \max \left\{s_{2}: s \in S\right\}\right) .
$$

For $B \in \mathbb{B}$ we also write $g(B)$ instead of $g(S(B)$ ). The Kalai-Rosenthal solution $K R$ (Kalai \& Rosenthal, 1978) assigns to each $S \in \mathbb{S}$ the maximal point of $S$ on the line segment with $(0,0)$ and $g(S)$ as endpoints. It is well defined because $S$ is compact. Clearly, $K R(S) \in P(S)$ for every $S \in \mathbb{S}$.

\subsection{Loss Aversion in Bargaining}

In this section we investigate the sensitivity of bargaining solutions towards changes in loss aversion of the bargainers.

Theorem 56 Let $B^{\lambda}=\left(A, \bar{a}_{2} C P T_{1}, C P T_{2}^{\lambda}\right)$ and $B^{\mu}=\left(A, \bar{a}, C P T_{1}, C P T_{2}^{\mu \mu}\right) \in \mathbb{B}$ where $B^{\lambda}$ and $B^{\mu}$ differ only in the loss aversion index of player 2. If $\lambda>\mu$ then

(i) $K R\left(B^{\lambda}\right) \leq K R\left(B^{\mu}\right)$,

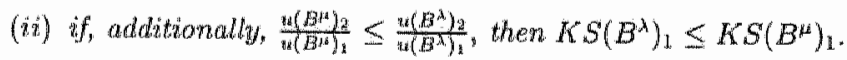

Remank 57 The condition in Theorem 56.(ii) is satisfied if the straight line through $(0,0)$ and $u\left(B^{\lambda}\right)$ is steeper than the straight line through $(0,0)$ and $u\left(B^{\mu}\right)$, which is ustually the case.

Proof of Theorem 56: The proof is elementary, and will only be sketched with the aid of pictures. 


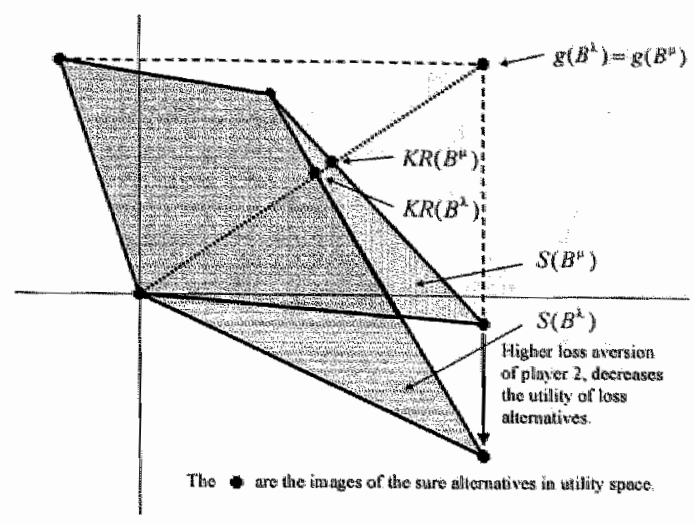

Figure 8.1: Theorem 56.(i)

(i) If $B^{\lambda}$ and $B^{\mu}$ differ only in the loss aversion index of player 2, we have $g\left(B^{\lambda}\right)=g\left(B^{\mu \lambda}\right)$. We know that $K R\left(B^{\lambda}\right) \in P\left(B^{\lambda}\right)$ and $K R\left(B^{\mu}\right) \in P\left(B^{\mu}\right)$, and that they both lie on the straight line through $(0,0)$ and $g\left(B^{\lambda}\right)$. Because of Observation 55 we have $K R\left(B^{\lambda}\right) \leq K R\left(B^{\mu}\right)$. See Figure 8.1. (Figure 8.1 displays a situation with linear weighting functions, so that the feasible sets are convex; for nonlinear weighting functions the feasible sets might be nonconvex.)

(ii) Suppose that $\frac{u\left(B^{\mu} \eta_{2}\right.}{u\left(B^{\alpha}\right)_{1}} \leq \frac{u\left(B^{\lambda}\right)_{2}}{u\left(B^{\lambda}\right)_{1}}$. Because of Observation 55, the straight line through $(0,0)$ and $u\left(B^{\mu}\right)$ intersects $P\left(B^{\lambda}\right)$ closer to $(0,0)$ than $P\left(B^{\mu}\right)$. The straight line trough $(0,0)$ and $u\left(B^{\lambda}\right)$ is steeper than the straight line through $(0,0)$ and $u\left(B^{\mu}\right)$, l.e. the straight line trough $(0,0)$ and $u\left(B^{\lambda}\right)$ intersects $P\left(B^{\lambda}\right)$ further to the left than the straight line trough $(0,0)$ and $u\left(B^{\mu}\right)$ does. Therefore, we have $K S\left(B^{\lambda}\right)_{\text {i }} \leq K S\left(B^{\mu}\right)_{1}$. See Figure 8.2 .

The extra condition $\frac{2\left(B^{N}\right)_{2}}{u\left(B^{x}\right)_{4}} \leq \frac{u\left(\theta^{A}\right)_{2}}{u\left(B^{X}\right)_{1}}$ in Theorem 56.(ii) cannot be ciropped, as the following example shows.

Example 58 Let $A=\{(0,0),(4,-1),(0,2),(0.6,0.4)\}, \bar{a}=(0,0), \mathbf{u}_{i}(a)=a_{i}$ for tail $a \in A, i \in\{1,2\}, \mu=1, \lambda=5, w_{1}^{+}(x)=w_{1}^{-}(x)=x$ for all $x \in[0,1], w_{2}^{+}(x)=0.2 * x$ for $x \in\left[0, \frac{1}{3}\right], w_{2}^{+}(x)=2.6 * x-0.8$ for $x \in\left(\frac{1}{3}, \frac{2}{3}\right), w_{2}^{+}(x)=0.2 * x+0.8$ for $x \in\left[\frac{2}{3}, 1\right]$, 


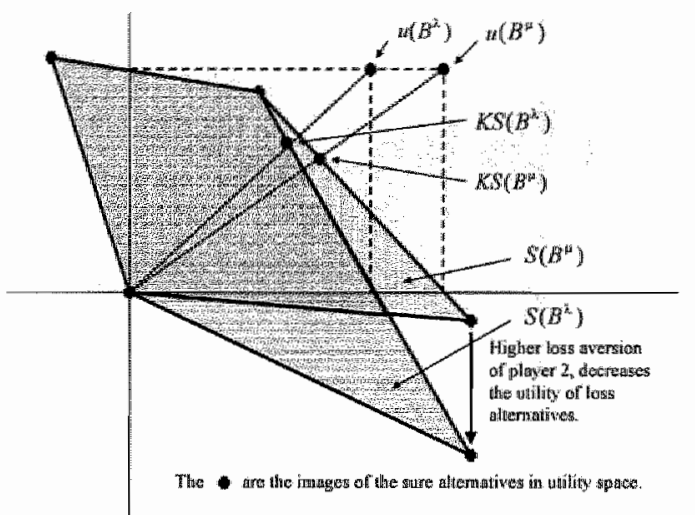

Figure 8.2: Theorem 56.(ii)

and $w_{2}^{-}(x)=1-w_{2}^{+}(1-x)$ for all $x \in[0,1]$. Let $C P T_{1}^{3}$ result from $\mathbf{u}_{1}, w_{1}^{+}, w_{1}^{-}$and loss aversion index $\mu$. Let $C P T_{2}^{\mu}$ and $C P T_{2}^{\lambda}$ result from $\mathbf{u}_{2}, w_{2}^{+,}, w_{2}^{-}$and loss aversion indices $\mu$ and $\lambda$, respectively. Let $B^{\mu}=\left(A, \bar{a}_{1} C P T_{1}, C P T_{2}^{\mu}\right)$ and $B^{\lambda}=\left(A, \bar{a}_{1} C P T_{1}, C P T_{2}^{\lambda}\right)$. Then $K S\left(B^{\mu}\right) \approx(0.69,0.47)$, but $K S\left(B^{\lambda}\right) \approx(0.84,0.42)$.

From Theorem 56 we know that under the Kalai-Rosenthal solution the more loss averse the opponent is, the worse it is for a player. This is also true in most cases under the Kalaj-Smorodinsky solution. An obvious question is, whether the opponent can have strategic advantages from being dishonest about the true loss aversion index.

First, we consider the question, whether player 2 can gain from pretending to be less loss averse. This means that if the true preferences can be represented by an evaluation function $C P T_{2}^{\lambda}$, the player announces preferences according to an evaluation function. $C P T_{2}^{\psi k}$ with $\mu<\lambda$. Because $C P T_{2}^{\lambda}$ represents the true preferences we have to evaluate the solutions of $B^{\mu}$ and $B^{\lambda}$ by $C P T_{2}^{\lambda}$ and to compare these two velues. It turns out that player 2 can never gain from pretending to be less loss averse as the following reasoning explains. By Theorem 56 Player 1 (weally) prefers the solution of $B^{\mu}$ over the solution of $B^{\lambda}$. Because of Pareto optimality of the solution of $B^{\lambda}$, Player 2 cannot strictly prefer the solution of $B^{\text {H4 }}$ over the solution of $B^{A}$. 
Can player 2 gain from pretending to be more loss averse? In many situations this is true, but the following example shows that this is not always the case. In this example the solutions of the two bargaining problems $B^{\lambda}$ and $B^{\mu}$ with $\mu<\lambda$ have to be evaluated by the true evaluation function $C P T_{2}^{\mu}$.

Example 59 Let $A=\left\{(0,0),(0,4),\left(4-\frac{4}{\sqrt{5}}, 0\right),(4,-1)\right\}, \bar{a}=(0,0), \mathrm{u}_{i}(a)=a_{i}$ for all $a \in A, i \in\{1,2\}, \mu=1, \lambda=4, w_{1}^{+}(x)=w_{2}^{-}(x)=x, w_{2}^{+}(x)=x^{2}$ and $w_{2}^{-}(x)=1-(1-x)^{2}$ for all $x \in[0,1]$. Let $C P T_{1}$ result from $u_{1}, u_{1}^{+}, w_{1}^{-}$and loss aversion index $\mu$. Let $C P T_{2}^{\gamma / R}$ and $C P T_{2}^{\lambda}$ result from $u_{2}, w_{2}^{+}, w_{2}^{-}$and loss aversion indices $\beta$ and $\lambda_{1}$ respectively. Let $B^{\mu}=\left(A, \bar{a}, C P T_{1}, C P T_{2}^{\mu}\right)$ and $B^{\lambda}=\left(A, \bar{a}, C P T_{1}, C P T_{2}^{\lambda}\right)$. The lotteries which give rise to the Kalai-Smorodinsky solution are $P^{\mu} \approx(0.75,(0,4) ; 0.25,(4 .-1))$ for $B^{\mu}$ and $P^{\lambda} \approx$ $\left(0.62,(0,4) ; 0.38,\left(4-\frac{4}{\sqrt{5}}, 0\right)\right.$ for $B^{\lambda}$. But $C P T_{2}^{\mu}\left(P^{\mu}\right) \approx 1.69>1.54 \approx C P T_{2}^{\mu}\left(P^{\lambda}\right)$.

\subsection{Conclusion}

This chapter has shown that it is often not advantageous to bargain aganst a more loss averse opponent. The intuition behind this result is that a more loss averse opponent is more demanding in obtaining compensation for possible losses.

The results can be generalized to $n$-person bargaining problems with the restriction that the feasible sets are made comprehensive. This guarantees that the solution acconding to $K R$ or $K S$ is at least weakly Pareto optimal. We omit the details. It is also possible to adapt the results to bargaining solutions exhibiting certain monotonicity properties, for instance the egalitarian bargaining sclution defined on the set of comprehensive problems.

The extension of the Nash bargaining solution (Nash, 1950) to bargaining problems under prospect theory is more problematic. In particular, feasible sets are not necessarily convex, so that the Nash bargaining solution is not always uniquely defined. Nevertheless, even if we restrict attention to bargaining problems where the Nash bargaining solution is well defined, it does not behave regulatly. Both cases can arise: a player can gain or lose with respect to the Nash bargaining solution when bargaining with a more loss averse opponent. 


\section{Chapter 9}

\section{An Empirical Test of Numeraire Illusion by Comparing Risk Attitudes Before and After the Introduction of the Euro}

The change of currency in Europe gave a unique opportunity to test whether people, when evaluating money, think in terms of numbers instead of in terms of real values. It is hard to disentangle the influence of numbers and real values on risk attitude. The currency conversion gave the opportunity to keep one of the two aspects fixed while the other varies, and to observe changes in risk attitude caused lyy changing numbers separately from that caused by changing real values.

In Belgium the scale changed by a, considerable, factor 40. Therefore, we chose Belginum to carry out an empirical study to test the effects of value versus numerical changes. We measured the risk attitudes of 87 subjects in December 2001, shortly belore the intro duction of the Euro, and did the same for 92 subjectes in May 2002, when people started to get accustomed to the Euro. We find that changes in value while keeping numbers constant do affect risk attitude, but changes in numbers while keeping values constant do noti, in agreement with postulates of rationality.!

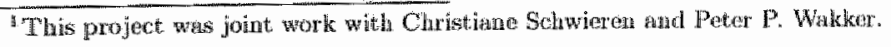




\subsection{Introduction}

A monetary amoumt is associated with two different values, its intrinsic and its nominal whe. The intrinsic value is its worth, for instance, $\$ 100$ is worth as much as 146 CHF (Swiss Francs), i.e. the intrinsic value of $\$ 100$ is the same as that of $146 \mathrm{CHF}$. The nominal walue $\$ 100$ is 100 , but the nominal value of $146 \mathrm{CHF}$ is 146 , i.e. the nominal values differ by the factor equal to the Dollar-Swiss Franc exchange rate.

The starting point of our research project was the question whether peoples exaluation of money in influenced more by intrinsic or more by nominal values. There is empirical evidence that people tend to think in nominal instead of intrinsic values (e.g. Shafir, Diamond \& Tverslky, 1997). Under rationality assumptions only intrinsic values should influence the risk attitudes of economic agents. Whether intrinsic values are expressed in Dollar, Swiss Franc or other currencjes should not change preferences: Dollar or Swiss Franc are only units of measurement. However, if people think in nominal values to a considerable extent, it is likely that the monetary unit, i.e. the currency, with respect to which intrinsic values are measured, does affect their risk attitudes. Glen Harrison (1994) called surch an effect mumeraire illusion.

Intrinsic and nominal vahues are difficult to separate, which makes it hard to disentangle their influence on risk attitude. If it were possible to keep one value fixed while the other value varies, then changes in risk attitude caused by the varying value could be observed separately from the influence of the other value. A simple idea is to measure and compare risk attitudes with respect to two different currencies as follows. First, the risk attitude can be compared for the same intrinsic values. Then, the different currencies obviously cause a change in the nominal values. Second, the risk attitude can be compared for the same nominal values. In this case, the intrinsic values differ. The strength of the differences in risk attitude in the two comparisons can provide a first answer to the question about the pelative impact of intrinsic and nominal values.

The problem is that in such an empirically experiment it can be difficult to control for distorting factors. For example, if risk atitudes are compared across different countries; cultural difleicences cannot be excluded. On the other hand, if an empirical study is conducted within one country, but with respect to different currencies, the problem is that there is always one reference currency. Two thinge can happen: Either the subjects al ways transform intrinsic values to the currency that they are used to and numeraire illusion cannot be observed, or the subjects "forget" that they do not deal with their own currency 
and answer the questions as if the numbers would correspond to their own currency causing some kind of myopic numeraire illusion. The latter form of myopic numeraire illusion is caused by inexperience. It is likely to happen, but we consider it as nninteresting and want to exclude it. Plott \& Sunder (1982) and Forsythe, Palfrey \& Plott (1982) deliberately took advantage of myopic numeraire illusion in experimental studies. In both papers, the experimental design was set up in French Francs instead of in American Dollars, although the experiments was carried out in the US. This was meant to make the subjects think in high norninal values, without making the experiment too expensive.

The introduction of the Euro gave a historically unique opportunity to test the dependence of risk attitudes on the unit of currency, by comparing risk attitudes before and after the introduction. Cultural differences can be exclucled, and also the described effect caused by inexperience can be excluded - at least if risk attitudes are elicited long enough after the introduction.

In the experiment, we first interviewed two clifferent groups of Belgian people before the introduction of the Euro in December 2001 and measured their risk attitudes with respect to Belgian Franc. We later did the same for two further groups of Belgians in May 2002 , i.e. four months after introduction of the Euro, and measured their risk attitude with respect to Eura. Each Belgian Franc group was matched by intrinsic values with one Euro group. Furthermore, one Belgian Franc group was matched by nominal values with one Euro group.

We have chosen Belgium because the exchange rate between Euro and former Belgian Franc is nearly exactly $1: 40$, that is 1 Euro is worth as much as 40 former Belgian Francs. One advantage of the exchange rate is that it is quite big, so that if numeraire illusion exists, we should be able to observe it. A second advantage is that round numbers remein round after the transformation. A further unforeseen advantage of Belgium is that it was the country with the highest overall acceptance and contentment for the Euro in May 2002 (see the Eurobarometer study by the European Commission of May 2002). Of course, we cannot exclude that our subjects still thought in their old currency to a considerable externt, but the subjects were young students and belong; therefore, to the demographic group which is supposed to be the fastest to get used to the new currency. All in all, it can be expected that our subjects already had a good feeling for the new currency when we carried out the second part of the experiment. 


\subsection{Method}

SUBAECT AND TREAMMENTS The experiment was conducted at the Unversity of Diepenbeek in Belgium. Participants were students of different faculties, who were recruited during their breaks in a meeting area of the miversity. We had four different treatments: A low- and a high-stimuli treatment for Belgian Franc (BF) conducted separately on two different days in December 2001, and a low- and a higli-stimuli treatment for Euro conducticl separately on two different days in May 2002. In each of the four treatments, between 43 and 47 subjects participated. We had about the same number of male and ferrale students in ench treatment.

Materual The study wes conducted as a paper/pencil study. The material consisted of one page of instructions in Dutch language and seven pages with one choice task on each pege. In each choice task the subjects were asked to decide whether they preferred to play a lottery with the chance to win some positive amount of money with a certain probability, or whether they preferred to obtain some lower amount of money for sure. The monetary amounts and the winning probabilities varied over the questions. The first two tasks served as learning exercises. In these two tasks it was very clear what the suggestive choice was. The order of the other five choice tasks varied over all participants to control for order-effects. The full questionmaine can be found in an English translation in the Appendix of the chapter.

The guestions for the low-stimuli Belgium Frane and the low-stimuli Euro treatments were identical with respect to intrinsic values, as were the questions for the high-stimuli Belgium Franc and the high-stimuli Euro treatments. Furthermore, the nominal values in the low-stimuli Belgium Franc treatment were identical with thase in the high-stimuli. Euro treatment. An example of the four versions of one of the choice tasks is as follows. What do you prefer:

$\begin{array}{ccc} & \text { A chance of } \frac{3}{4} \text { to win } & \text { for sure? } \\ \text { Low BF } & 600 \mathrm{BF} & 400 \mathrm{BF} \\ \text { Low Euro } & 15 \mathrm{Euro} & 10 \text { Euro } \\ \text { High BF } & 24,000 \mathrm{BF} & 16,000 \mathrm{BF} \\ \text { High Euro } & 600 \text { Euro } & 400 \text { Euro }\end{array}$


It can be seen that the numbers for the low-stimuli Belgian Franc treatment are equal to those in the high-stimuli Euro treatment. The numbers in the two low-stimuli treatments differ by the Euro-BF exchange rate of a factor 40 , as do the numbers for the two highstimuli treatments.

The average gain over the seven choice tasks was about 5 Euro (i.e. $200 \mathrm{BF}$ ) for the low-stimuli questions and 200 Euro (i.e. $8000 \mathrm{BF}$ ) for the high-stimuli questions. Due to budget constraints, the subjects in the high-stimuli treatments had a chance of only $1: 20$ to play one of their choices for real money. In order to have a similar mechanism for the low-stimuli treatments we decided to give those subjects a chance of $1: 2$ to play one of their choices for real money.

Procedure Two experimenters asked students sitting in the meeting area of the University of Diepenbeek whether they were willing to participate in a 10 minute experiment. When students agreed to participate, they received a booklet with one page of instructions, seven pages with the choice questions, and a final page asking for demographics and giving: information how to contact us. Participants were asked to work quietly and individually. After filling in the booklet, it was determined for each subject whether the subject got the chance to play one of the choices for real money. This was done by means of a 20-sided die. The low-stimuli treatment subjects had a chance of $1 / 2$ to play, and were asked to guess whether the die would come up with an even or an odd number. The high-stimuli treatment subjects had a chance of $1 / 20$ to play, and were asked to guess, which number of the die would cone up. The subjects that were selected to play one of the choices for real money, were then asked to draw one of seven cards that determined, which of the seven choices was played. In case that in the corresponding choice task the sure amount was chosen, we payed that amount, in case that the lottery was chosen, we carried out the lottery by means of the 20-sided die again.

\subsection{Results}

To determine the risk attitude of each subject we counted the number of lottery choices excluding the two learning tasks. Then, the four different treatments can be easily compared by an independent samples t-test. The following tables sunmarize the observed datia. We do not find any gender differences in risk aversion. 
Belgian Franc low-stimuli treatment:

$\begin{array}{lllllll}\text { Number of lottery chaices } & 0 & 1 & 2 & 3 & 4 & 5 \\ \text { Frequency } & 1 & 7 & 7 & 13 & 11 & 4 \\ \text { Percent } & 2.3 & 16.3 & 16.3 & 30.2 & 25.6 & 9.3 \\ \text { Cumulative Percent } & 2.3 & 18.6 & 34.9 & 65.1 & 90.7 & 100\end{array}$

Belgian Franc high-stimuli treatment:

$\begin{array}{lllllll}\text { Number of lottery choices } & 0 & 1 & 2 & 3 & 4 & 5 \\ \text { Frequency } & 1 & 15 & 21 & 2 & 5 & 1 \\ \text { Percent } & 2.2 & 33.3 & 46.7 & 4.4 & 11.1 & 2.2 \\ \text { Cumulative Percent } & 2.2 & 35.6 & 82.2 & 86.7 & 97.8 & 100\end{array}$

Euro low-stimuli treatment:

$\begin{array}{lllllll}\text { Number of lottery choices } & 0 & 1 & 2 & 3 & 4 & 5 \\ \text { Frequency } & 2 & 8 & 1.2 & 15 & 10 & 1 \\ \text { Percent } & 4.2 & 16.7 & 25.0 & 31.3 & 20.8 & 2.1 \\ \text { Cumulative Percent } & 4.2 & 20.8 & 45.8 & 77.1 & 97.9 & 100\end{array}$

Euro high-stimuli treatment:

$\begin{array}{lllllll}\text { Number of lottery choices } & 0 & 1 & 2 & 3 & 4 & 5 \\ \text { Frequency } & 4 & 7 & 17 & 13 & 4 & 0 \\ \text { Percent } & 8.9 & 15.6 & 37.8 & 28.9 & 8.9 & 0 \\ \text { Cumulative Percent } & 8.9 & 24.4 & 62.2 & 91.1 & 100 & 100\end{array}$

Usually, if the utility over money for agents is elicited empirically, increasing relative risk aversion is found. It can be explained as follows. Assume that $x$ it the positive amount that makes an agent indifferent between obtaining that amount for sure and playing a lottery with the chance of winning a bigger amount $y$ with probability $p$ and nothing otherwise. Then, the amount $z$ that makes the agent indifferent between obtaining $z$ for sure and playing a lottery with the chance of winning $a * y$ with probability $p$, where $a>1$, is usually smaller than $a * x$. That is, for higher monetary amounts the agents are relatively more risk averse.

Because of increasing relative risk aversion we expect to find more risk aversion for the high-stimuli Belgian Franc treatment than for the low-stimuli Belgian Franc treatment. The difference is significant, indeed (one-tailed: $t_{86}=3.679_{*} p=0.000$ ). For the same reason we expect to find more risk aversion for the high-stimuli Euro treatment than for the 
low-stimuli Euro treatment. Again, this is confirmed by the data (one-tailed: $t_{91}=1.735$, $p=0.043)$.

For the comparison of the two low-stimuli treatments and for the two high-stimuli treatments (in these comparisons intrinsic values are the same, but nominal values differ), the data does not give evidence for a difference in risk attitude, neither for the low- nor for the high-stimuli treatments (low-stimuli treatment two-tailed: $t_{89}=1.316, p=0.191$; high-stimuli treatment two-tailed: $t_{88}=-0.787, p=0.434$ ).

The final interesting comparison is that of the low-stinuli Belgian Franc versus the high-stimuli Euro treatment. In these two treatments the nominal values (lout not the intrinsic values) are identical. It turns out that we find strong evidence for a difference (two-tailed: $t_{86}=2.959, p=0.004$ ).

\subsection{Conclusion}

For the comparison of the groups with identical intrinsic values but different nominall values, we do not find a difference in risk attitude, suggesting that nominal values have no or little influence on the risk attitude. We find a very significant difference for the comparison of the groups with identical nominal values but different intrinsic values. From this, we can conclude that intrinsic values have a strong influence on risk altitude. To summarize, we find that changes in intrinsic value while keeping nominal values constant do affect risk attitude, but changes in nominal values while keeping intrinsic values constant do not.

The data obtained from our empirical study do not give evidence for a change in risk aversion caused by the currency conversion from Belgian Franc to Euro. If numeraire illusion were an important psychological factor, we would expect such a change in risk aversion. Therefore, we conclude that if mumeraire illusion exists, it only plays a minor role - at least as long as the subjects are experienced with the currency under conisiceration. In the domain that we considered up to about 2000 Euro the subjects seern to be aware of the intrinsic values behind the numbers, no matter whether the currency is Euro or Belgian Franc. Maybe numeraire illusion only becomes infuential for much higher monetary amounts, when the subjects loose a good, intuitive feeling for the intrinsic values. 


\subsection{Appendix}

THE INSTRUCTIONS IN AN ENGLISH TRANSLATION

For each of the four treatments the instruction were more or less the same, only one sentence differed between the lowand the high-stimuli treatments. The instruction for the low-stimuli treatments are given hereafter, the deviating sentence for the high-stimuli treatments is given in brackets II.

\section{Research Project on Opinions over Uncertain Payments}

In this research project we are interested in opinions over uncertain payments. Therefore, we will offer you sever choice situations, in which you each time have to choose between obtaining some amount of money with certainty or playing a lottery. In such a lottery you can win some monetary amount with a certain probability, otherwise you win nothing. You can only win money, you never loose money. There are no right or wrong answers in these questions, only your own preferences count. This is also what we are interested in the research project!

- On every of the following seven pages you find a monetary amount that you can get with certainty, and a lottery over money. You are asked to state what you prefer: that you obtain the sure amount of that the lottery is played. Mark your preference each time!

- Later it will be determined if one of your choices will really be played. For this you are asked to guess whether an even or an odd number comes up when you throw a 20-sided die. [For this you are asked to guess which number comes up when you throw a 20 -sided die.] If you guess wrong, the experiment is over for you, and you won unfortunately nothing. If you guess right then one of your marked choices will really be carried out.

- After that, you pick one of seven numbered cards to determine from which page your choice will be carried out.

- Fron the selected page you get the sure amount in case you marked the sure amount, and we play the lottery in case you marked the lottery. 
As already said before, there are no right or wrong answers, and we are interested in your own preferences. It is also the best for yourself to mark on every page your true preference. After all, if a page is selected than what you marked will be carried out.

THE CHOICE TASKS On the following seven pages the seven choice tasks can be found. For each choice task we present the four different version for the different treatments. Each time, the left column gives the choice tasks for the low-stimuli treatments, and the right column those for the high-stimuli treatments. The upper row gives the choice tasks for the Belgian Franc treatments, the lower row those for the Euro treatments. The first two choice task are the learning questions. 

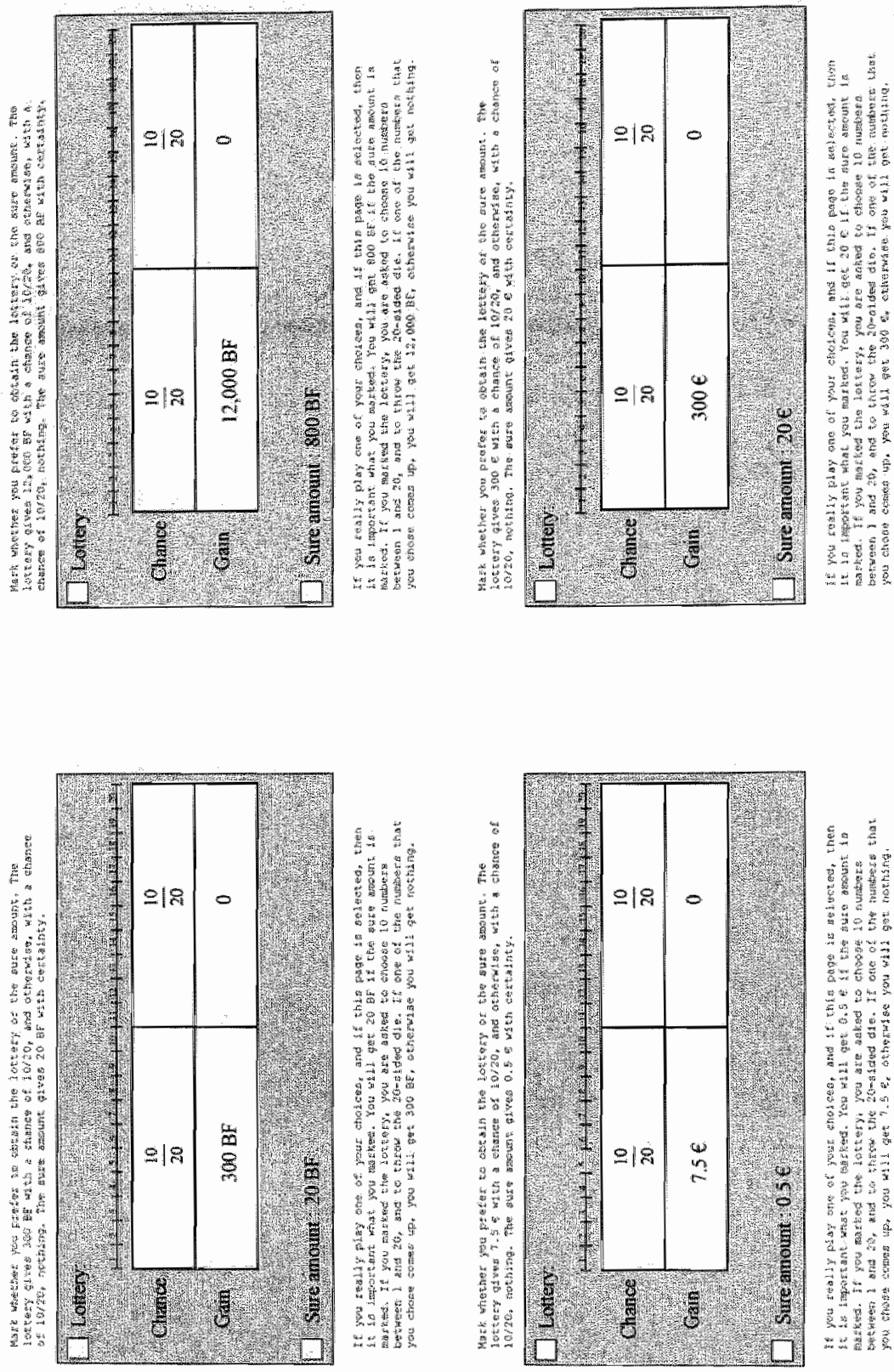

שู

단

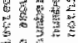

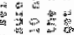

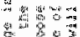

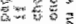

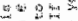

sog.

y.

की

6.

क

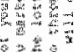

in

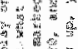

a

要的

3.

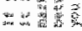



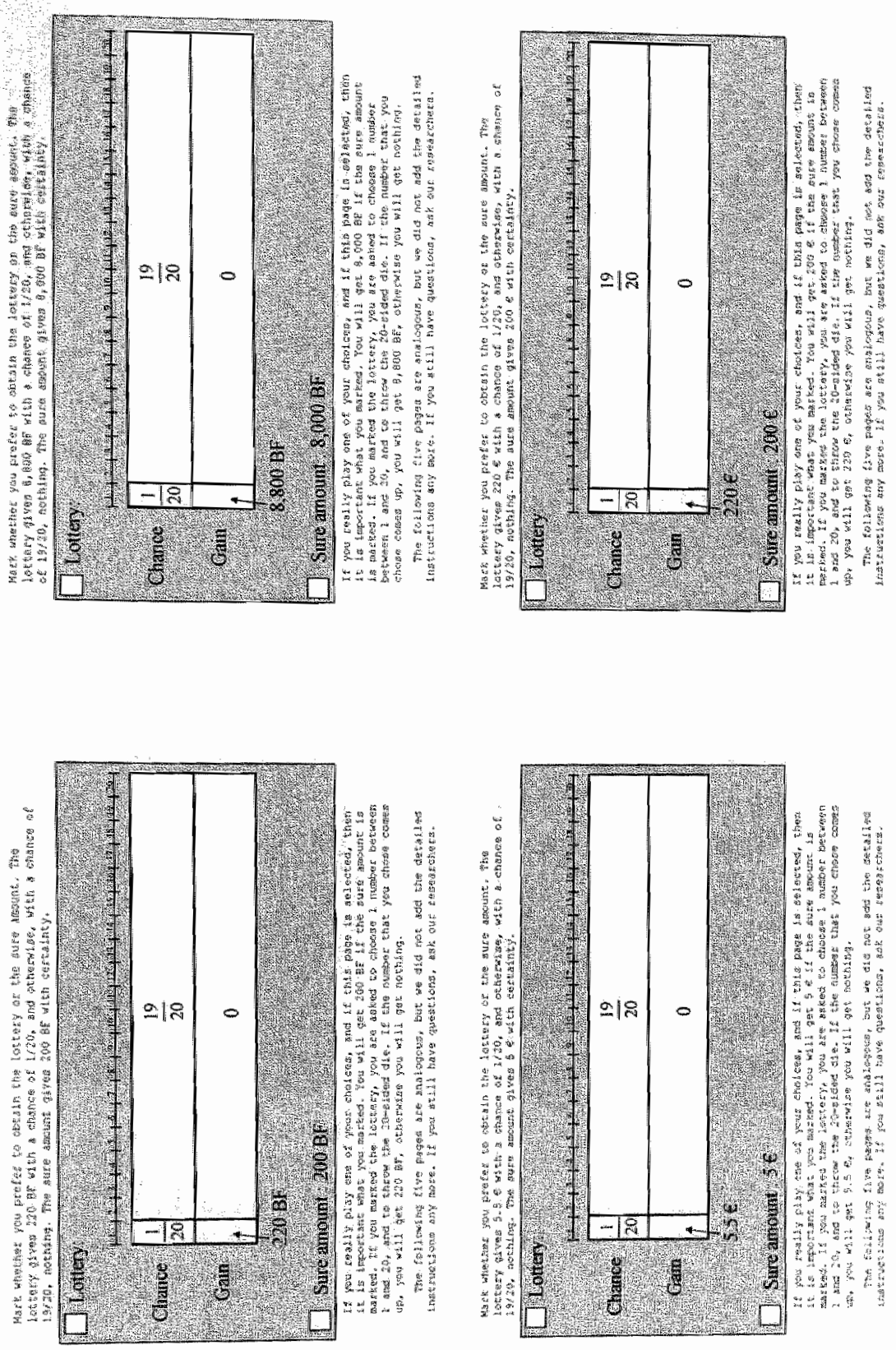

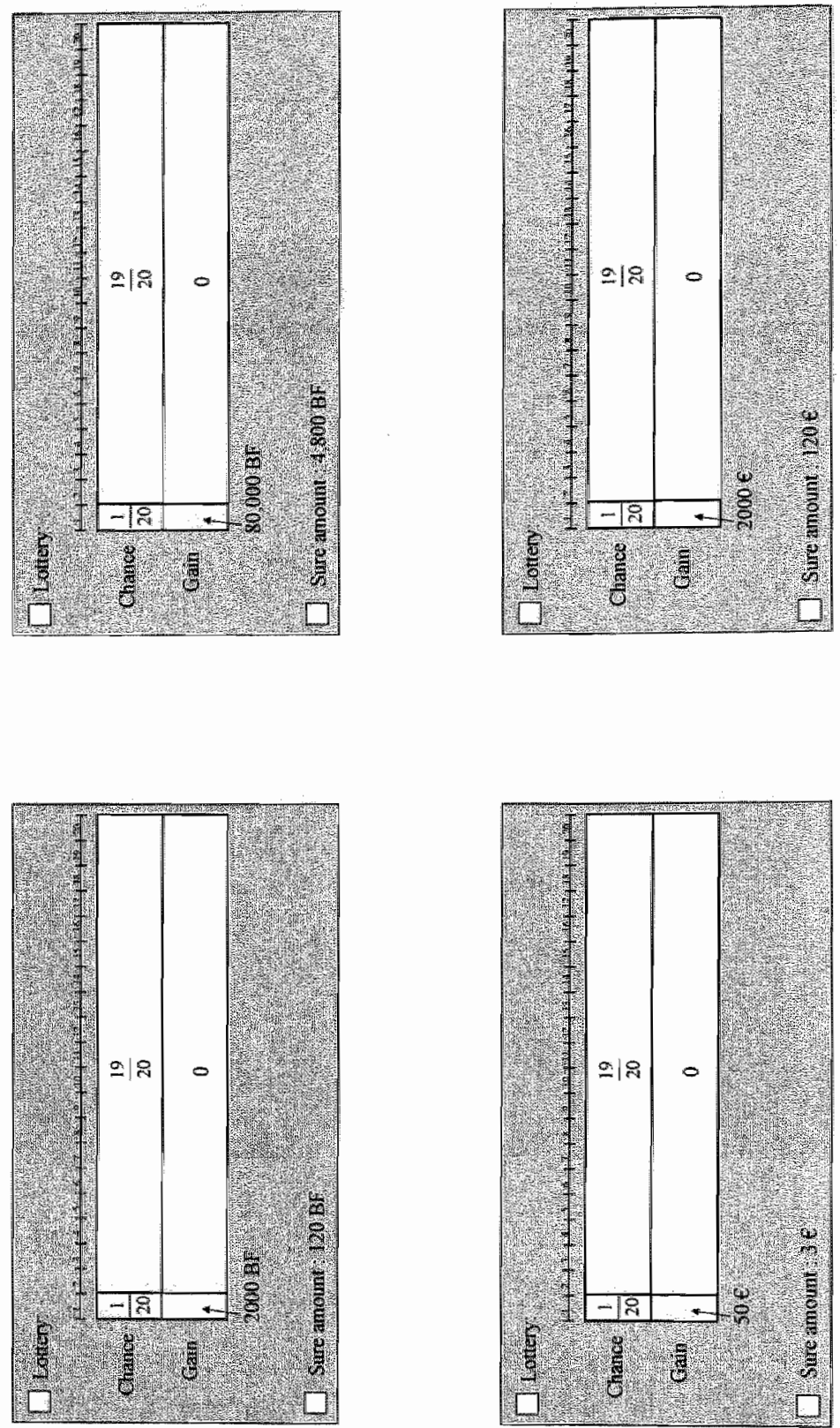

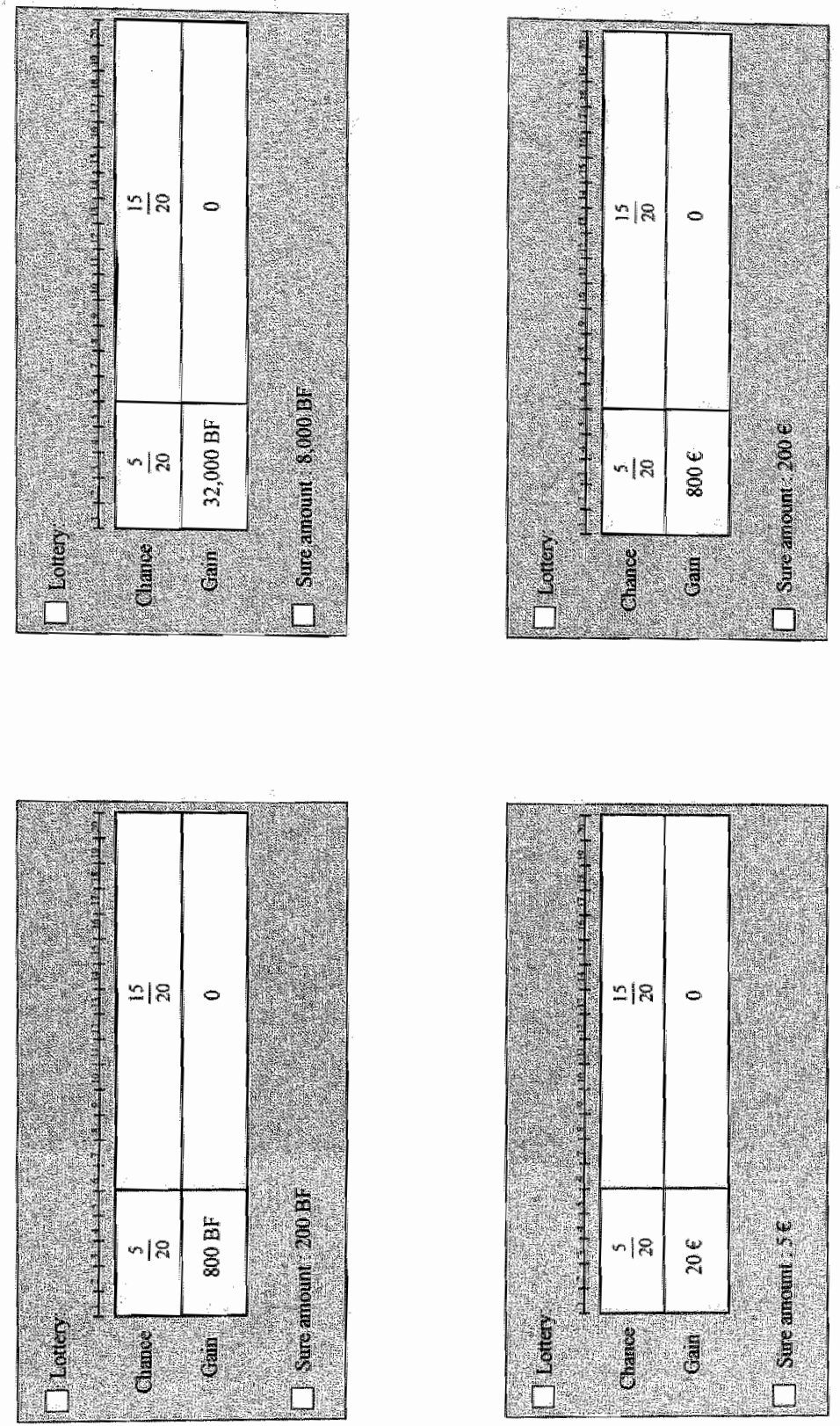

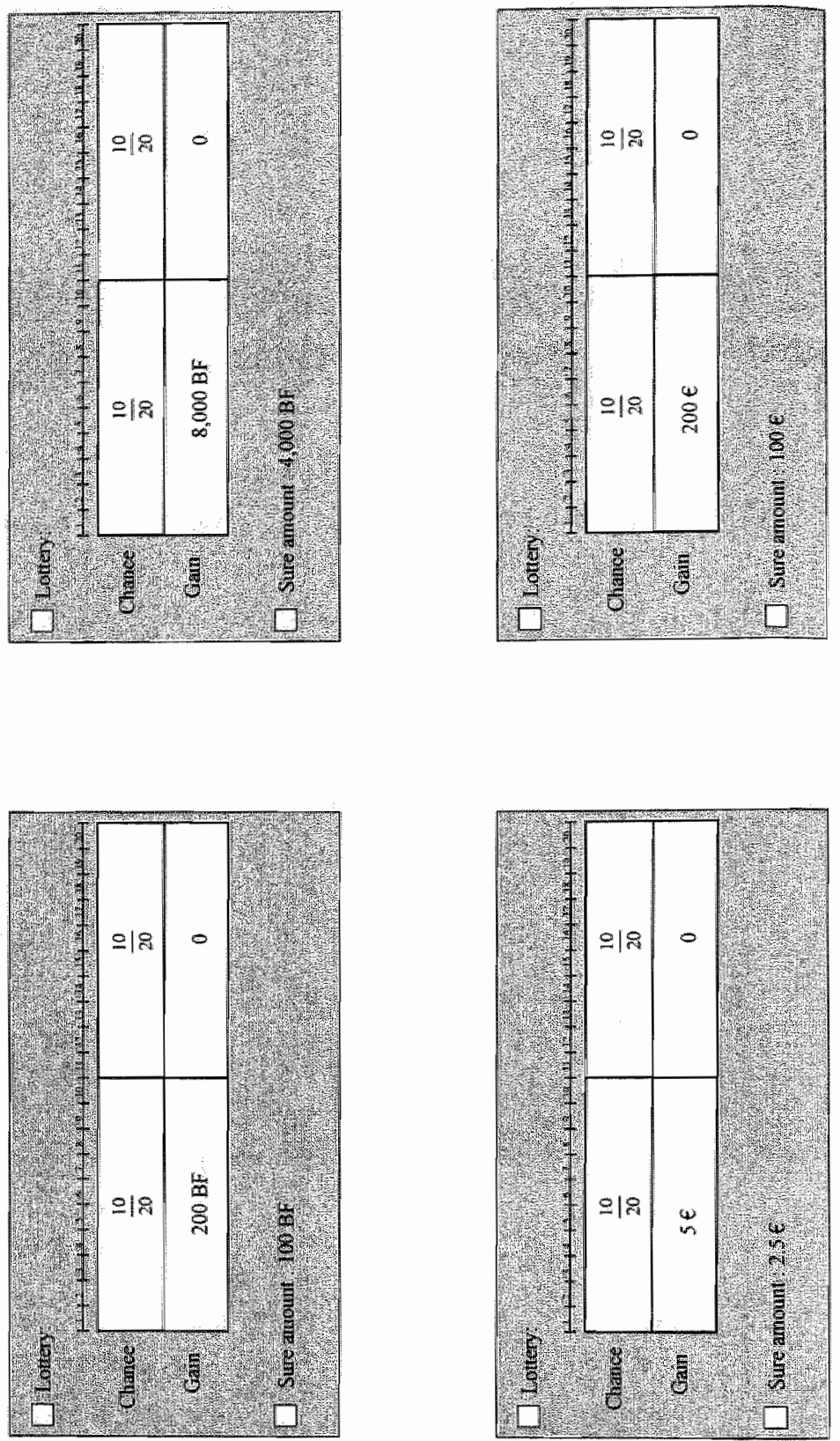

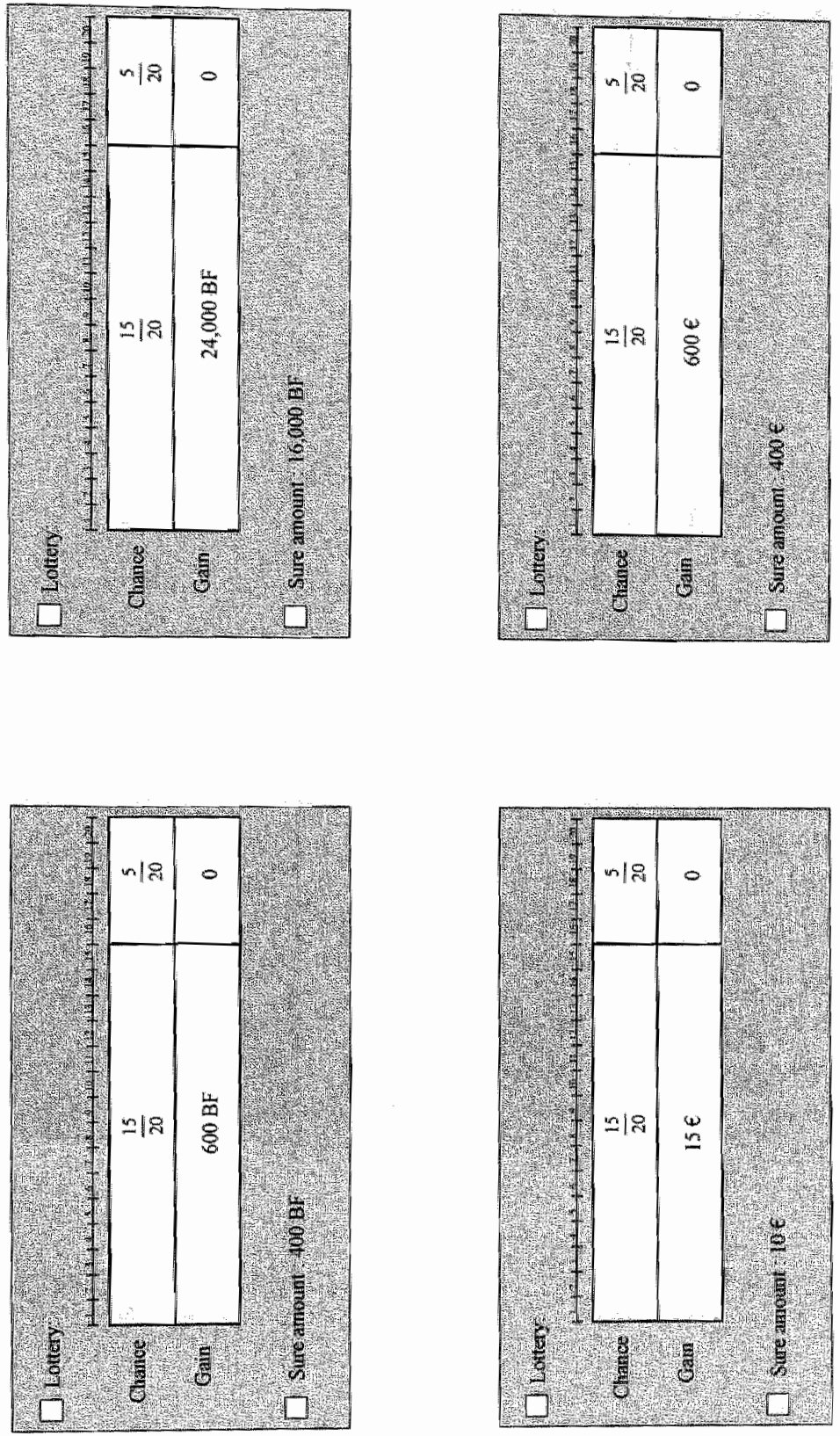

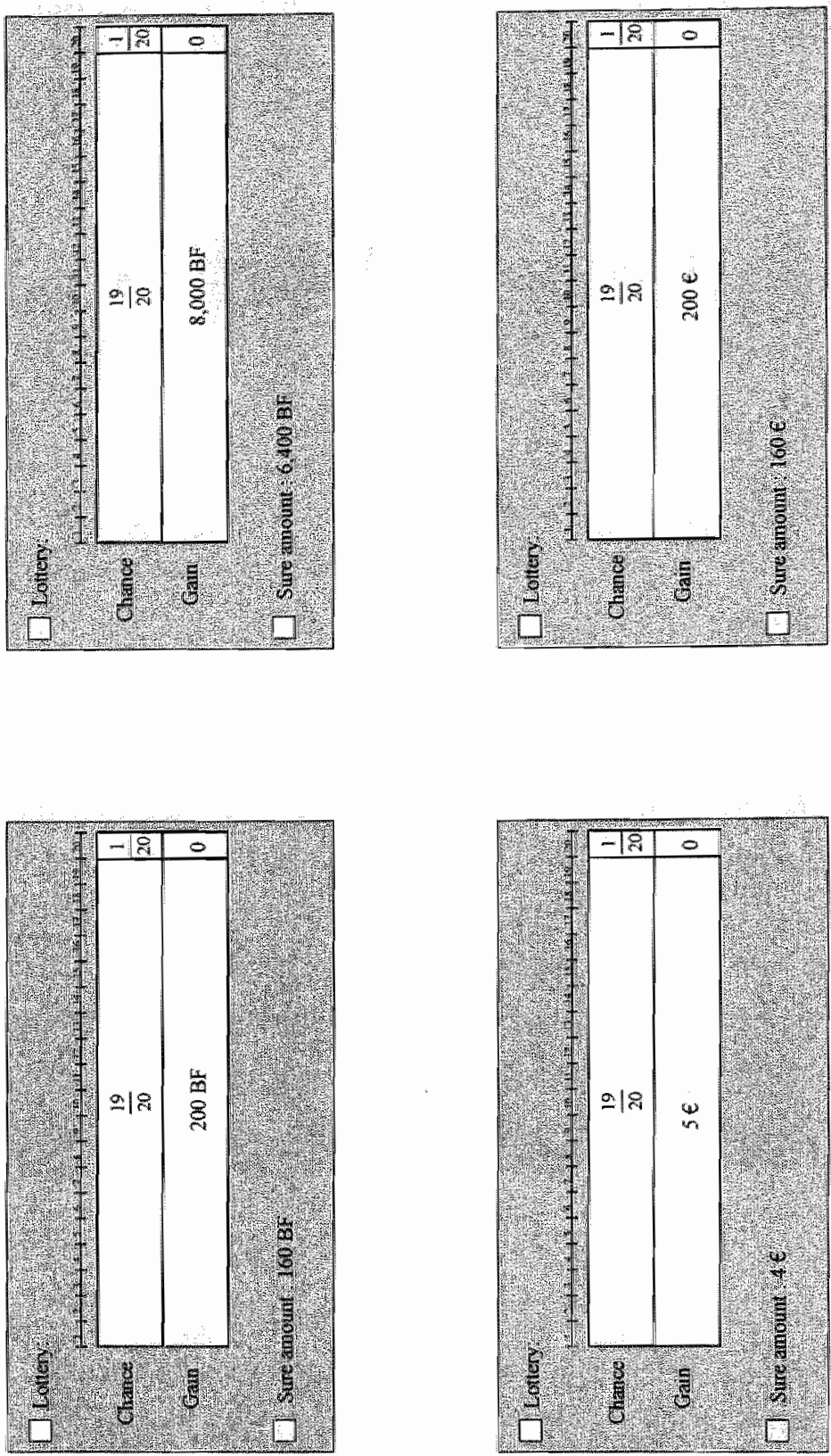


\section{References}

Abdellaoui, Mohammed (2000), "Parameter-Free Elicitation of Utilities and Probability Weighting Functions," Management Science 46, 1497-1512.

Abdellaou, Mohammed (2002), "A. Genuine Rank-Dependent Generalization of the von Neumann-Morgenstern Expected Utility Theorem," Econometrica70, 717-737.

Abclellaoui, Mohammed, Carolina Barrios, \& Peter P. Wakker (2002), "Reconciling Introspective Utility With Revealed Preference: Experimental Arguments Based On Prospect Theory," GRID, Cachan, France.

Abdellaoui, Mohammed \& Bertrand R. Munier (2001), "Testing Consistency of Probability Tradeoffs in Individual Decision-Making under Risk," GRID, Cachan, France.

Adams, Ernest W., Robert F. Fagot, \& Richard E. Robinson (1970), "On the Empirical Status of Axioms in Theories of Fundamental Measurement," Journal of Mathematical Psychology 7, 379-409.

Allais, Maurice (1953), "Fondements d'une Theorie Positive des Choix Comportant un Risque et Critique des Postulats et Axiomes de l'Ecole Américaine," Colloques Internationaux du Centre National de la Recherche Scientifique 40, Econométrie, 257-332. Paris: Centre National de la Recherche Scientifique. Translated into English, with additions, as "The Foundations of a Positive Theory of Choice Involving Risk and a Criticism of the Postulates and Axioms of the American School," in Maurice Allais \& Ole Hagen (1979, Eds.), Expected Utility Hypotheses and the Allais Paradox, 27-145, Reidel, Dordrecht, The Netherlands. 
Alt, Franz (1936), "Uber die Messbarkeit des Nutzens." Zeitschrift fur Nationalökonomie 7, 161-169. Translated into English by Siegfried Schach (1971), "On the Measurability of Utility." In John S. Chipman, Leonid Hurwicz, Marcel KK. Richter, \& Hugo F. Sonnenschein (Eds.), Preferences, Utility, and Demand, Hartcourt Brace Jowanowich, New York, Chapter 20.

Anscombe, F.J. \& Robert J. Aumann (1963), "A Definition of Subjective Probability", Annals of Mathematical Statistics 34, 199-205.

Arrow, Kenneth J. (1951), "Alternative Approaches to the Theory of Choice in Risk-Taking Situations," Econometrica 19, 404-437.

Arrow, Kenneth J. (1953), "Le Róle des Valleurs Boursières pour la Répartition la Meilleure des Risques." Collocues Internationaux du Centre National de la Recherche Scientifique (Econométrie) 40, 41 47. Translated into English as "The Role of Securities in the Optimal Allocation of Risk-Bearing," Review of Economic Studies 31 (1964), 91-96.

Arrow, Kenneth J. (1965), "Aspects of the Theory of Risk-Bearing." Academic Bookstore, Helsinki. Elaborated as Arrow, Kenneth J. (1971), "Essays in the Theory of Risk-Bearing." North-Holland, Amsterdam.

Barberis, Nicholas, Ming Huang, \& Tano Santos (2001), "Prospect Theory and Asset Prices," Quarterly Journal of Economics 66, 1-53.

Baron, Jonathan (1994, second edition), "Thinking and Deciding." Cambridge University Press, Cambridge.

Basu, Kaushik (1982), "Determinateness of the Utility Function: Revisiting a Controversy of the Thirties," Review of Economic Studies 49, 307-311.

Bateman, Ian J. Alistair Murro, Bruce Rhodes, Chris Starmer, \& Robert Sugden (1997), "A Test of: the Theory of Reference-Dependent Preferences," The Quarterly Journal of Economics 112, 647-661.

Benartzi, Shlomo \& Richard H. Thaler (1995), "Myopic Loss Aversion and the Equity Premium Puzzle," The Quarterly Journal of Economics 110, 73-92. 
Bentham, Jeremy (1789), "The Principles of Morals and Legislation." At the Clarendon Presis, Oxford.

Bernoulli, Damiel (1738), "Specimen "Theoriae Novae de Mensura Sortis," Commentari Academiae Scientiarum Imperialis Petropolitanae 5, 175 192. Translated into English by Louise Sommer (1954), "Exposition of a New Theory on the Measurement of Risk" " Econometrica 22, 23-36; or in Alfed N. Page (Ed., 1968), "Utility "Theory: A Book of Readings," Chapter 11, Wiley, New York.

Bewley, Truman F. (1986), "Knightian Decision Theory Part I," Cowles Foundation Discussion Paper No. 807.

Bleichrodt, Han \& John Miyamoto (2002), "A Characterization of Quality-Adjusted LifeYears under Cumulative Prospect Theory," Mathematics of Operations Research, forthcoming.

Bleichrodt, Han \& Jose Luis Pinto (2000), "A Parameter-Free Elicitation of the Probability Weighting Function in Medical Decision Making," Management Science 46, 1485-1496.

Bleichrodt, Han \& Jose Luis Pinto (2001), "The Validity of QALYs under Nonexpected Utility, iMTA, Erasmus University, Rotterdam, The Netherlands.

Bleichrodt, Han, Jose Luis Pinto, \& Peter P. Wakker (1999), "Making Descriptive Use of Prospect Theory to Improve Prescriptive Applications of Expected Utility, Management. Science 47, 1498-1514.

Bleichrodt, Han \& John Quiggin (1997), "Characterizing QALYis under a General Rank Dependent Utility Model," Journal of Risk and Uncertainty 15, 151-165.

Bleichrodt, Han \& John Quggin (1999), "Life Cycle Preferences over Consumption and Health: When is Cost-Effectiveness Analy is Equivalent to Cost-Benelit Analysis?" Journal of Healtih Economics $18,681-708$.

Bonman, David, Deborah Minehart, \& Matthew Rabin (1999), "Loss Aversion in at Consumption-Savings Model," Journal of Economic Behavior and Organization 38, 155178 . 
Breiter, Hans C., Ithak Aharon, Daniel Kahneman, Anders Dale, \& Peter Shizgal (2001), "Functional Imaging of Neural Responses to Expectancy and Experience of Monetary Gains and Losses," Neuron 30, 619-639.

Broome, John R. (1991), "Weighing Goods." Basil Blackwell, Oxford.

Burgos, Albert, Simon Grant, \& Atsushi Kajii (2002), "Bargaining and Boldness," Games and Economic Behavior 38, 28-51.

Cachon, Gerard P. \& Colin F. Camerer (1996), "Loss-Avoidance and Forward Induction in Experimental Coordination Games," Quarterly Journal of Economics 111, 165-194.

Camerer, Colin F. (1995), "Individual Decision Making." In John H. Kagel \& Alvin E. Roth (Eds.), Handbook of Experimental Economics, 587-703, Princeton University Press, Princeton, NJ.

Chateauneuf, Alain (1991), "On the Use of Capacities in Modeling Uncertainty Aversion and Risk Aversion," Journal of Mathematical Economics 20, 343-369.

Chateauneuf, Alain (1.999), "Comonotonicity Axioms and Rank-Dependent Expected Utility Theory for Arbitrary Consequences," Journal of Mathematical Economics 32, 21-45.

Chateauneuf, Alain \& Michèle Cohnen (1994), "Risk Seeking with Diminishing Marginal Utility in \& Non-Expected Utility Model," Journal of Risk and Uncertainty 9, 77-91.

Chateauneuf, Alain, Thibault Gajdos \& Pierre-Henry Wilthien (2002), "The Principle of Strong Diminishing Transfer," Journal of Economic Theory 103, 311-333.

Chateauneuf, Alain \& Jean-Marc Tallon (2002), "Diversification, Convex Preferences and Non-Empty CORE," Economic Theory 19, 509-523.

Chatenuneuf, Alain \& Peter P. Wakker (1999), "An Axiomatization of Cumulative Prospect Theory for Decision under Risk," Joumal of Risk and Uncertainty $18,137-145$.

Chew, Soo Hong (1989), "The Rank-Dependent Quasilinear Mean" Unpublished manuscript, Department of Economics, University of California, Irvine, USA.

Chew, Soo Hong \& Edi Kami (1994), "Choquet Expected Utility with a Finite State Space: Commutativity and Act-Independence," Jcurnal of Economic Theory 62, 469-479. 
Chew, Soo Hong, Edi Karmi, \& Zwi Safra (1987), "Risk Aversion in the Theory of Expected Otility with Rank Dependent Probabilities," Joumal of Economic Theory 42, 370-381.

Chew, Soo Hong \& Amos Trersy (1990), "Chmulative Prospect Theory: ReferenceDependent Axiomatization of Decision under Uncertainty." In preparation (newer conpleted), Stanford University; presented by Tversky at the 5th Foundations of Utility and Risk (FUR) conference, Duke University, Durham NO, 1990.

Cowen, Tyler (1992), "Weighing Goods: Equality, Uncertainty, and Time," Book Review of: John R. Broome (1991), "Weighing Goods," Basil Blackwell, Oxford, UK; Economics and Philosophy 8, $283-284$.

Crewford, Vincent P. \& Hal $\mathbb{R}$. Varian (1979): "Distortion of Preferences and the Nash "Theory of Bargaining," Econormics Letters 3, 203-206.

Davidson, Donald \& Jacob Marschak (1956), "Experimental Tests of Stochastic Decision Theory." In C. West Churchman \& Philburn Ratoosh (Eds.), Measurement: Definitions. and Theories, Willey, New Yonk.

Debreu, Gérard (1958), "Stochastic Choice and Cardinal Utility" Econometrica 26, 440444

Debreu, Gerard (1960), "Topological Methods in Cardinal Utility Theory." In Kenneth J. Arrow, Samuel Karlia, \& Patrick Suppes (1960, Eds..), Mathematical Methods in the Social Sciences, 16-26, Stanford University Press, Stanford, CA.

de Finetti, Bruno (1937), "La Prévision: Ses Lois Logiques, ses Sources Subjectives," Annales de l'Institut Henri Poincaré 7, 1-68. Translated into English by Henry E. Kyburg, "Foresight: Its Logical Laws, its Subjective Sources." In Henry E. Kyburg \& Howard E. Smokler (1964, Eds.), Studies in Subjective Probability, Wiley, New York, 53-118; 2nd edition 1980, Krieger, New York.

Dekel, Eddie (1986), "An Axiometic Characterization of Preferences under Uncertanty: Weakening the Independence Axiom," Jounal of Econonic Theory $40,304-318$.

Ebent, Udo (2000), "Social Welfare, Inequality, and Poverty when Neods Differ: RankDependent Evaluation Functions," Dept. of Economics, University of Oldenburg, Germaxy. 
Ellsberg, Daniel (1961), "Risk, Ambiguity and the Savage Axioms," Quarterly Journal of Economics $75,643-669$.

Epstein, Larry G. (1992), "Behavior under Risk: Recent Developments in Theory and Applications." In Jean-Jacques Laffont (Ed.), Advances in Economic Theory, 1-63 Carnbridge University Press, Cambridge UK.

Eurobarometer study by the European Commission in May 2002, hittp://europa.eu.int/comm/public_opinion/flash/fl121_3_in_en.pdf.

Farquhar, Peter H. \& L. Robin Keller (1989), "Preference Intensity Measurement," Annals of Operations Research 19, 205-217.

Fennema, Hein \& Marcel A.L.M. van Assen (1998), "Measuring the Utility of Losses by Means of the Tradeoff Method," Journal of Risk and Uncertainty 17, 277-295.

Fishburn, Peter C. (1970), "Utility Theory for Decision Making." Wiley, New York.

Fishburn, Peter C. (1977), "Mean-Risk Analysis with Risk Associated with Below-Target Returns," American Economic Review 67, 116-126.

Fishburn, Peter C. (1982), "The Foundations of Expected Utility." Reidel, Dordrecht.

Fishburn, Peter C. (1988), "Nonlinear Preference and Utility Theory." Johns Hopkins University Press, Baltimore, MD.

Fishburn, Peter C. (1989), "Retrospective on the Utility Theory of von Neumann and Morgenstern," Journal of Risk and Uncertainty 2, 127-158.

Fishburn, Peter C. (1992), "Utility as Additive Set Function," Mathematics of Operations Research $17,910-920$.

Fishburn, Peter C. \& Irving H. La Valle (1988), "Context-Dependent Choice with Nonlinear and Nontransitive Preferences," Econometrica 56, 1221-1239. 
Frisch, Ragnar (1926), "Sur un Probleme d'Economie Pure," Norsk Matematisk Foremings. Skrifter Serie 1 16, 1-40. Translated into English by John S. Chipman, "On a Problem in. Pure Economics." In John S. Chipman, Leonid Hurwicz, Marcel K. Richter, Hugo F. Sommenschein (1971, Eds.), Preferenoes, Utillity, and Demand, Chapter 19, Hartcourt, New York.

Forsythe, Robert, Thomas R. Palfey and Charles R. Plott (1982), "Asset Valuation in an Experimental Mar"ket," Econometrica 50, 537-568.

Ghirardato, Paolo, Fabio Maccheroni, Massimo Marinacci, \& Marciano Siniscalch (2001), "Subjective Foundations for Objective Randomization: A New Spin on Roulette Wheels," Economic Dept, University of Bollogna.

Ghirardato, Paolo \& Messimo Marinacci (2001), "Risk, Ambiguity, and the Separation of Utility and Beliefs," Mathematics of Operations Research $26,864-890$.

Ghirardato, Paolo \& Massimo Marimacci (2002), "Ambiguity Made Precise: A Comparative Foundation," Journal of Economic Theory 102, 251-289.

Gilboa, Itzhak (1987), "Expected Utility with Purely Subjective Non-Additive Probabilities," Journal of Mathernatical Economics 16, 65-88.

Gilboa, Itzhak (1989), "Expectations and Variations in Multi-Period Decisions," Econometrica $57,1153-1169$.

Gilboa, Itzhak \& David Schmeidler (1989), "Maxmin Expected Utiliby with a Non-Unique Prior" Journal of Mathematical Economics 18, 141-153.

Gilboa, Itzhak \& David Schimeider (2001), "A Cognitive Model of Individual Well-Being", Social Choice and Welfare 18, $569-288$.

Gilboa, Itzhak, David Schmeidler, \& Peter P. Wakker (2002), "Utility in Case-Based Decision Theory," Journal of Economic "Theory 105, 483-502.

Gneezy, Uri \& $J a n$ Potters (1997), "An Experiment on Risk Taking and Evaluation Periods," The Quarterly Joumal of Economics 112, 631-645. 
Grodal, Birgit (1978), "Some Further Results on Integral Representation of Utility Functions;" Institute of Economics, University of Copenhagen, Copenhagen.

Gul, Faruk (1992), "Sawage"s Theorem with a Finite Number of States," Journal of Economic "Theory 57, 99-110. ("Erratum," Journal of Economic Theory 61, 1993, 184.)

Harrison, Glen W. (1994), "Expected Utility Theory and the Experimentalists," Empirical Economics 19, 223-253.

Harsanyi, John C. (1955), "Cardinal Welfare, Individualistic Ethics, and Interpersonal Comparisons of Utility," Joumal of Political Economy 63, 309-321.

Harsanyi, John C. (1978), "Bayesian Decision Theory and Utilitarian Ethics," American Economic Review, Papers and Proceedings 68, 223-228.

Heath, Chip, Steven Huddart, \& Mark Lang (1999), "Psychological Factors and Stock Option Exercise," Quarterly Journal of Economics 114, 601-627.

Hershey, Jolun C., Howard C. Kunreuther, \& Paul J.H. Schoemaker (1982), "Sources of Bias in Assessment Procedures for Utility Functions," Management Science 28, 936-953.

Hershey, John C. \& Paul J.H. Schoemaker (1985), "Probability versus Certainty Equivalence Methods in Utility Measurement," Management Science 31, 1213-1231.

Hicks, John R. \& Roy G.D. Allen (1934), "A Reconsideration of the Theory of Value: I; II," Economica 1, 52-75; 196-219.

Holthausen, Duncan M. (1981), "A Risk-Return Model with Risk and Return Measured as Deviations from a Target Return," American Economic Review 71, 182-188.

Huber, Joel, Dan Ariely, \& Gregory Fischer (2001), "Expressing Preference in a PrincipalAgent Task: A Comparison of Choice, Rating, and Matching," Organizational Behavior and Human Decision Processes 87, 66-90.

Kahmeman, Daniel (1994), "New Challenges to the Rationality Assumption," Journal of Institutional and Theoretical Economics 150, 18-36. 
Kahneman, Daniel, Jack L. Knetsch, \& Richard H. Thaler (1991), "The Endowment Bffect, Loss Aversion, and Status Quo Bias: Amomalies," Joumal of Economic Perspectues 5 , $193-206$.

Kahneman, Daniel \& Amos Tvensky (1979), "Prospect Theory: An Analysis of Decision under Risk," Econometrica 47, 263-291.

Kalai, Ehud \& Meir Smorodinsky (1975): "Other Solutions to Nash's Bargaining Problem," Econometrica 43,513-518.

Kalai, Ehud \& Robert W. Rosenthal (1978): "Arbitration of Two-Party Disputes Under" Ignorance," International Joumal of Game Theory 7, 65-72.

Kannai, Yakkar (1977): "Concavifiability and Constructions of Concave Utility Functions," Journal of Mathematical Economics 4, 1-56.

Karni, Edi (2001), "On the Representation of Beliefs by Probabilities," The Wolns Hopkins University, Department of Political Economy.

Karmi, Edi \& Zvi Safra (1998), "Hexagon Condition and Additive Representation of Preferences: An Algebraic Approach," Joun nal of Mathematical Psychology 42, 393- 399.

Karni, Edi \& Zvi Safra (2002), "Individual Sense of Justice: A Utility Representation," Econometrica 70, 263-284.

Kami, Fdi \& David Schmeidler (1991), "Utility Theory with Uncertainty." In Werner" Hildenbrand \& Hugo F. Somenschein (Eds), Handbook of Mathematical Enonomics 4 , Chapter 33, 1763-1831, North-Holland, Ansterdam.

Kihlstrom, Richard E., Alvin E. Roth, \& David Schmeidlel (1981): "Risk Aversion and Solutions to Nash's Bargaining Problem," in: O. Moeschlin and D. Pallaschke (Ceds), Gume Theory and Mathematical Economics. Amsterdam: North Holland.

Kobberling, Veronika (2002a), "Strength of Preference and Cardinal Utility," University of Maasticht, The Netherlands.

Kobberling, Veronika (2002b), "Comments on: Edi Karni \& Zvi Safra (1998), The Hexagon Condition and Additive Representations for "Two Dimensions: An Algebraic Approach," Journal of Mathematical Psychology, forthcoming. 
Kobblyerling, Verontka \& Hans Peters (2002): "The Effect of Decision Weights in Bargaiming Problems;" Jommal of Fconomic Theory, forthcoming.

Kobblberling, Veronilka 8 Hans Petert (2001): "Loss Aversion in Bargaining Problems," University of Maasticht. The Netherlands.

Kóbberling, Veronika \& Peter P. Wakker (2002a)," A Tool for Qualitatively Testing, Quantitatively Measuring, and Normatively Justifying Expected Utility, University of Maastricht, The Netherllands.

Kabberling, Veronika \& Peter P. Walker (2002b), "Preference Foundations for Nonexpected Utility: A Generalized and Simplified Technique," Mathematics of Operations Research, forthcoming.

Kobbberling, Veronika \& Peter P. Wakker (2002c), "An Index of Loss Aversion," University of Marasticht, The Netherlands.

Krantz, David H., R. Duncan Luce, Patrick Suppes, \& Amos Tversky (1971), "Foundations of Measurement, Voll. I. (Additive and Polynomial Representations)." Academic Press, New York.

LeRoy, Stephen F. \& Jan Werner (2000), "Principles of Financial Economics." Cambriclge University Press, New York.

Loomes, Graham \& Robert Sugden (1982), "Regret Theory: An Alternative Theory of Rational Choice under Uncertainty," Economic Journal 92, 805-824.

Loomes, Graham \& Robert Sugden (1986), "Disappointment and Dynamic Consistency in Choice under Uncertainty," Review of Economic Studies 53, 271-282.

Luce, R. Duncan (1988), "Rank-Dependent, Subjective Expected-Utility Representations," Journal of Risk and Uncertainty $1,305-332$.

Luce, R. Duncan (1991), "Rank- and-Sign Dependent Linear Utility Models for Binary Gambles," Joumal of Economic Theory 53,75-100.

Luce, R. Duncean (1998), "Coalescing, Event Commutativity, and Theories of Utility" Journall of Risk and Uncertainty $16,87-114$. 
Luce, R. Duncan (2000), "Utility of Gains and Losses: Measurement-Theoretical and Experimental Approaches." Lawrence Eribanm Publishers, London.

Luce, R. Duncan \& Peter C. Fishburn (1991), "Rank- and Sign-Dependent Linear Utility Models for Finite First-Order Gambles," Journal of Risk and Uncertainty 4, $29-59$.

Luce, R. Duncan, David H. Krantz, Patrick Suppes, \& Amos Tversky (1990), "Foundations of Measurement, Vol. III. (Representation, Axiomatization, and Invariance." Academic Press, New York.

Machina, Mark J. (1982), "Expected Utility" Analysis without the Independence Axiom," Econometrica 50, 277-323.

Markowitz, Harry M. (1952), "The Utility of Wealth," Journal of Political Economy 60, $151-158$.

Mendelson, Bert (1962), "Introduction to Topology." Dover, New York. (3rd ed. 1990).

Merton, Robert C. (1971), "Optimum Consumption and Portfolio Rules in a ContinuousTime Model," Journal of Economic Theory 3, 373-413.

Miyamoto, John M. (1988), "Generic Utility Theory: Measurement Foundations and Applications in Multiattribute Utility Theory," Journal of Mathematical Psychology 32, 357404.

Münnich, "Akos, Gyula Maksia, \& Robert J. Mokken (2000), "n-Variable Bisection," Journal of Mathematical Psychology 44, 569-581.

Nakamura, Yutaka (1990), "Subjective Expected Utility with Non-Addlitive Probabilities on Finite State Spaces," Journal of Economic Theory 51, 346-366.

Nakamura, Yutaka (1992), "Multi-Symmetric Structures and Non-Expected Utility," Jour" nal of Mathematical Psychology 36, 375-395.

Nakamura, Yutaka (1995), "Rank Dependent Utility for Arbitrary Consequence Spaces," Mathematical Social Sciences 29, 103-1.29.

Narens, Louis (1985), "Abstract Measurement Theory." MLT Press, Cambridge, MA. 
Nash, John F. (1950): "The Bargaining Problem," Econometrica 18, 155-162.

Neilson, Wiliam S. (2002), "Comparative Risk Sensitivity with Reference-Dependent Preferencess," Journal of Risk and Uncertainty 24, 131-142.

$\mathrm{Ng}$, Yew-Kwang (1997), "A Case for Happiness, Cardinalism, and Interpersonal Compatrability," Economic Journal 107, 1.848-1858.

Pareto, Vilfredo (1906), "Manuele di Economia Politica," Piccolo Biblioteca Scientifica Milan. Translated into French in 1927 as "Manuel d 'Economie Politique," Giard, Paris, second edition. Translated into English by Ann S. Schwier (1971),"Manuel of Political Economy, "MacMillan, London.

Payne, John W., James R. Bettman, \& David A. Schkade (1999), "Measuring Constructed Preferences: Towards a Building Code," Journal of Risk and Uncertainty 19, 243-270.

Payne, John W., Dan J. Laughhunn, \& Roy L. Crum (1980), "Translation of Gambles and Aspiration Level Effects in Risky Choice Behavior," Management Science 26, 1039-1060.

Payne, John W., Dan J. Laughhumn, \& Roy L. Crum (1981), "Further Tests of Aspiration Level Effects in Risky Behavior," Management Science 27, 953-958.

Peters, Hans (1992): "A Criterion for Comparing Strength of Preference, with an Application to Bargaining;" Operations Research 40, 1018-1022.

Pfanzagl, Joham (1959), "A General Theory of Measurement -Applications to Utility;" Naval Research Logistics Quarterly 6, 283-294.

Pfanzagl, Johamn (1968), "Theory of measurement," New York: Wiley, 1968.

Pratt, John W. (1964), "Risk Aversion in the Small and in the Large," Econometrica 32, 122-136.

Prelec, Drazen (1998), "The Probability Weighting Function," Econometrica 66, 497-527. (First version: Prelec, Drazen (1989): "On the Shape of the Decision Weight Function," Harvard Business School, Harvard University, Cambridge, MA, USA.) 
Plott, Charles R. \& Shyam Sunder 1982, "Enffiency of Experimental Security Markats with Insider Information: Am Application of Rational-Expectations Models;" Journal of Political Economy 90, 663m698.

Quiggin, John (1981), "Risk Perception and Risk Aversion among Australian Farmers," Australian Joumal of Agricullural Ecomomics 25, 160-169.

Quiggin, John (1982): "A Theory of Anticipated Utility," Economic Behavior and Organization $3,324-344$.

Rabin, Matthew (2000), "Risk Aversion and Expected-utility Theory: A Calbration The orem?" Econometrica 68, 1281-1292.

Rabin, Matthew \& Richard H. Thaler (2001), "Anomalies: Risk Aversion," Joumal of" Economic Perspectives 15, 219-232.

Raiffa, Howard (1968), "Decision Analysis" Addison-Wesley, London.

Ramsey, Frank P. (1931), "Truth and Probability." In "The Foundations of Mathematics and other Logical Essays," 156-198, Routledge and Kegan Paul, London. Reprinted in Henry E. Kyburg \& Howard E. Smokler (1964, Eds.), Studies in Subjective Probabiliby, 61-92, Wiley, New York.

Rabson, Arthur J. (2001), "Why Would Nature Give Individuals Utility Functions?", The Journal of Political Economy 109, 900-914.

Roth, Alvin E. \& Uriel G. Rothblum (1982)" "Risk Aversion and Nash's Solution for Bargaining Games with Risky Outcomes," Econometrice 50, 639-647.

Rothschil, Michael \& Joseph E. Stiglitz (1970): "Increasing Risk: 1. A Definition," Journal of Economic Theory 2, 225-243.

Rubinstein, Ariel, Zvi Safta, \& Willam Thomson (1992): "On the Interpretation of the Nash Bargaining Solution and its Extension to Non-expected Utility Preferences," Econco. metrica $60,1171-1186$.

Safra, Zw, Lin Zhou, \& Itzhak Zilcha (1990)" "Risk Aversion in the Nash Bargaining Problem with Risky Outcomes and Risky Disagreement Points," Econometrica 58, 961 965. 
Safra, Zvi \& Itzhak Zilcha (1993): "Bargaining Solutions without the Expected Utility Hypothesis," Games and Economic Behavior 5, 288-306.

Samuelson, Paul A. (1937), "A Note on the Measurement of Utility," Review of Economic Studies 4, 155-161.

Samuelson, Paul A. (1938), "The Numerical Representation of Ordered Classifications and the Concept of Utility;" Review of Economic Studies 6, 65-70.

Sarin, Rakesh K. (1982), "Strength of Preference and Risky Choice," Operations Research $30,982-997$.

Sarin, Rakesh K. \& Peter P. Wakker (1992), "A Simple Axiomatization of Nonadditive Expected Utility," Econometrica 60, 1255-1272.

Savage, Leonard J. (1954), "The Foundations of Statistics." Wiley, New York. (Second edition 1972, Dover, New York.)

Schmeidler, David (1989), "Subjective Probability and Expected Utility without Additivity," Econometrica 57, 571-587.

Schmidt, Ulrich (1999), "Alternatives to Expected Utility: Some Formal Theories." In Salvador Barberâ, Peter J. Hammond, \& Christian Seidl, Handbook of Utility Theory, Kluwer Academic Publishers, Dordrecht, in press.

Schmidt, Ulrich (2002), "Reference-Dependence in Cumulative Prospect Theory," Dept. of Finance, Christian-Albrechts-University, Kiel, Germany.

Schmidt, Ulrich \& Stefan Traub (2000), "An Experimental Test of Loss Aversion," Dept. of Finance, Christian-Albrechts-University, Kiel, Germany.

Schmidt, Ulrich \& Horst Zank (2001a), "A New Axiomatization of Rank-Dependent Expected Utility with Tradeoff Consistency for Equally Likely Outcomes," Journal of Mathematical Economics 35, 483-491.

Schmidt, Ulrich \& Horst Zank (2001b), "An Axiomatization of Linear Cumulative Prospect Theory with Applications to Portfolio Selection and Insurance Demand," School of Economic Studies, The University of Manchester. 
Schmidt, Uhrich \& Horst Zank (2002, in preparation), "Risk Aversion in Cumulative Prospect Theory," School of Economic Studies, "The University of Manchester.

Scott, Dana \& Patrick Suppes (1958), "Foundational Aspects of Theories of Measurement" Journal of Symbolic Logic 23, 113-128.

Segal, Uzi (1989), "Anticipated Utility: A Measure Representation Approach" Annals of Operations Research 19, 359-373.

Segal, Uzi (1993a), "The Measure Representation: A Cornection," Jommal of Risk and Uncertainty $6,99-107$.

Segal, Uzi (1993b), "Order Indifference and Rank-Dependent Probabilities," Joumal of Mathematical Economics 22, 373-397.

Sen, Amartya K. (1993), "The Internal Consistency of Choice," Econometrica 61, 495-521.

Shafir, Eldar, Peter A. Diamond, \& Amos Tversky (1997), "Money Illusion," Quarterly Journal of Economics 112, 341-374.

Shalev, Jonathan (1997): "Loss Aversion and Bargaining," CORE Discussion Paper.

Shalev, Jonathan (2000), "Loss Aversion Equilibrium," International Journal of Game Theory 29, 269-287.

Shapley, Lloyd S. (1975), "Cardinal Utility Comparisons from Intensity Comparisons." Report R-1683-PR, The Rand Corporation, Santa Monica, California.

Šipoš, Ján (1979), "Integral with Respect to a Pre-Measure," Math. Slovaca 29, 141-155.

Skiadas, Costis (1997), "Subjective Probability under Additive Aggregation of Conditional Preferences, Joumal of Economic "Theory $76,242-271$.

Sobel, Joel (1981): "Distortion of Utilities and the Bargaining Problem," Econometrica $49,597-619$

Sobel, Joel (2001): "Manipulation of Preferences and Relative Utilitarianism," Geunes and Economic Behavior: $37,196-215$. 
Stamer, Chris (2000), "Developments in Non-Expected Utility Theory: The Hunt for a Descriptive Theory of Choice under Risk" Journall of Economic Literature 38, 332-382.

Stigler, George J. (1950), " The Development of Utility Theory: I; II," Journal of Political Economy 58, 307-327; 373-396. Reprinted in Alfred N. Page (1968), Utility Theory: A Book of Readings, Wiley, New York, 55-119.

Suppes, Patrick \& M. Winet (1955), "An Axiomatization of Utility Based on the Notion of Utility Differences," Management Science 1, 259-270.

Suppes, Patrick \& Joseph L. Zirnes (1963), "Basic Measurement Theory." In R. Duncan Luce, Robert R. Bush, \& Eugene Galanter (Eds.), Handbook of Mathematical Psychology, Vol. 1, 1-76, Wiley, New York.

Thaler, Richard H, Amos Twersky, Daniel Kahneman, \& Alan Schwartz (1997), "The Effect of Myopia and Loss Aversion on Risk Taking: An Experimental Test," The Quarterly Journal of Economics 112, 647-661.

The Economist (2001), "Economics Focus Averse to Reality," The Economist, August 11, p. 61 .

Tinbergen, Jan (1991), "On the Measurement of Welfare," Journal of Econometrics 50, 7-13.

Tversky, Amos (1969), "Intransitivity of Preferences," Psychological Review 76, 31-48.

Twersky, Amos (1977), "Features of Similarity," Psychological Review 84, 327-352.

Tversky, Amos \& Daniel Kahmeman (1991), "Loss Aversion in Riskless Choice: A Reference Dependent Model," Quarterly Journal of Economics 106, 1039-1061.

Tversky, Amos \& Daniel Kahneman (1992), "Advances in Prospect Theory: Cumulative Representation of Uncertainty," Journal of Risk and Uncertzinty 5, 297-323.

Tversky, Amos, Shmuel. Sattath, \& Paul Slovic (1988), "Contingent Weighting in Judgment and Choice," Psychological Review 95, 371-384.

Vind, Karl (1991), "Independent Preferences," Journal of Mathematical Economics 20, $1.19-135$. 
Volij, Oscar \& Eyal Winter (2000): "On Risk Aversion and Bargaining Outcomes," Games and Economic Behavior 41, 120-140.

von Neumam, John \& Oskar Morgenstern (1944, 1947, 1953), "Theory of Games and Economic Behavior." Princeton University Press, Princeton.

Wakker, Peter P. (1984), "Cardinal Coordinate Independence for Expected Utility" Journal of Mathematical Psychology 28, 110-117.

Wakker, Peter P. (1988), "The Algebraic versus the Topological Approach to Additive Representations," Journal of Mathematical Psychology 32, 421-435.

Wakker, Peter P. (1989), "Additive Representations of Preferences, A New Foundation of Decision Analysis," Kluwer Academic Publishers, Dordrecht.

Wakker, Peter P. (1990), "Under Stochastic Dominance Choquet-Expected Utility and Anticipated Utility are Identical," Theory and Decision 29, 119-132.

Wakker, Peter P. (1991a), "Additive Representation for Equally Spaced Structures," Journal of Mathematical Psychology 35, 260-266.

Wakker, Peter P. (1991b)," Additive Representations on Rank-Ordered Sets. I. The Algebraic Approach," Journal of Mathematical Psychology 35, 501-531.

Wakker, Peter P. (1991c), "Additive Representations of Preferences, A New Foundation. of Decision Aralysis; The Algebraic Approach." In Jean-Paul Doignon \& Jean-Claude Falmagne (Eds.), Mathematical Psychology: Current Developments, Springer, Berlin, 7187.

Wakker, Peter P. (1993a), "Additive Representations on Rank-Ordered Setis II. The Topological Approach," Jounmal of Mathematical Ecomomics 22, 1-26.

Wakker, Peter P. (1993b), "Unbounded Utility for Savage"s "Foundations of Statistics" and other Models," Mathematics of Operations Research 18, 446-485.

Wakker, Peter P. (1994), "Separating Marginal Utility and Probabilistic Risk Aversion," Theory and Decision $36,1-44$. 
Wakker, Peter P. \& Daniel Deneffe (1996), "Eliciting von Neumann-Morgenstern Utilities when Probabilities Are Distorted or Unknown," Management Science 42, 1131-1150.

Wakker, Peter P. \& Amos Tversky (1993), "An Axiomatization of Cumulative Prospect Theory," Journal of Risk and Uncertainty 7, 147-176.

Wakker, Peter P. \& Horst Zank (1999), "A Unifed Derivation of Classical Subjective Expected Utility Models through Cardinal Utility;" Journal of Mathematical Economics $32,1-19$.

Weymark, John A. (1981), "Generalized Gini Inequality Indices," Mathematical Social Sciences 1, $409-430$.

Yaari, Menahem E. (1969), "Some Remarks on Measures of Risk Aversion and on Their Uses: $_{3}$, Journal of Economic Theory 1, 315-329.

Yaari, Menahem E. (1986): "Univariate and Multivariate Comperisons of Risk Aversion: A New Approach," in: W. P. Heller, R. M. Starr, and D. A. Starrett (eds.), Uncertainty, Information and Communication, Essays in Honor of Kenneth J. Arrow, Vol. III, 173-187, Cambridge University Press, Cambridge.

Yaari, Menahem E. (1987), "The Dual Theory of Choice under Risk," Econometrica 55, $95-115$.

Zank, Horst (2001), "Cumulative Prospect Theory for Parametric and Multiattribute Utilities," Mathematics of Operations Research 26, 67-81.

Zeuthen, Frederik (1937), "On the Determinateness of the Utility Function," Review of Economic Studies 4, 236-239. 


\section{Author Index}

Abdellaoui, Mohammed, 28, 29, 35-37, $44,55,57,87$

Adams, Ernest W., 62

Aharon, Ithak, 85

Allais, Maurice, 28,29

Allen, Roy G.D., 5, 30

Alt, Franz, 6, 7, 14

Anscombe, F.J., 46, 56

Ariely, Dan, 85

Arrow, Kenneth J., 83

Aumann, Robert J., 46, 56

Barberis, Nicholas, 81, 83, 93

Baron, Jonathan, 30,35

Barrios, Carolina, 29, 37

Basu, Kaushik, 14

Bateman, Ian J., 83,84

Benartzi, Shlomo, $76,78,82,85$

Bentham, Jeremy, 37

Bernoulli, Daniel, 79

Betiman, James R., 36

Bewley, Truman F. 53

Bleichrodt, Han, 35, 36, 44, 56, 57, 83-85

Bowman, David, 85

Breiter, Hans C., 85

Broome, John R, 29, 30, 36, 37

Burgos, Albert, 99
Cachon, Gérard P., 83

Camerer, Colin F, 6, 83

Chateauneuf, Alain, 53, 54, 56, 76, 79, 98, 101

Chew, Soo Hong, 29, 55-57, 79, 98

Cohen, Michèle, 79, 98, 101

Cowen, Tyler, 84

Crawford, Vincent $\mathrm{P}_{\text {. }}, 99$

Crum, Roy L., 83

Dale, Anders, 85

Davidson, Donald, 14

de Finetti, Bruno, 27, 47, 56

Debreu, Gérard, 14

Dekel, Eddie, 29

Deneffe, Daniel, 28, 35, 44

Diamond, Peter A., 132

Ebert, Udo, 35, 57

Ellsberg, Daniel, 28

Epstein, Larry G., 28, 29

Fagot, Robert F., 62

Farquhar, Peter H., 6

Fennema, Hein, 36, 44, 87

Fischer, Gregory, 85

Fishburn, Peter $\mathrm{C}^{4} 29,31,32,49,35,84$

Forsythe, Robert, 133

Frisch, Ragnar, 6 
Gajdos, Thibault, 76

Ghirardato, Paolo, 53,56, 57,61

Giboa, ltohak, $6,29,35,39,44,53,55$

Gneezy, Uri, 83

Gront, Simon, 99

Grodal, Birgit, 29

Gul, Faruk, 29, 56, 57

Harrison, GHen, 132

Harsany:, John C., 6, 29, 39

Heath, Chip, 87

Hershey, John C., 83, 84

Hicks, John R., 5,30

Holthausen, Duncan M., 84

Huang, Ming, 81, 83, 93

Huber, Joel, 85

Huddard, Steven, 87

Kahneman, Daniel, $6,28,35,36,40,44$, $57,75,77,78,82-87,89-91,111$, 122,123

Kajii, Atsushi, 99

Kalai, Ehud, 99, 103, 109-111, 122, 123, 125,126

Kannai, Yakkar, 98, 121

Karni, Edi, 9, 10, 24, 29, 35, 55, 57, 79, 84, 98

Keller, $\mathrm{I}_{\text {i. }}$ Robin, 6

Kililstrom, Richard E, 98, 99, 121

Knetsch, Jack L., 82, 122

Firantz, David $\mathrm{H}, 10,12-15,22$

Kranta, David FI., 40, 43, 45, 59-62, 70, 72,73

Kunrenther, Howard C., 83, 84
Lang, Marti, 87

Laughhum, Dan $₫, 83$

LaValle, Irving $\mathrm{H} ., 32$

LeRoy, Stephen $\mathbb{E}$, 28, 57

Loomes, Grabm, 6, 28-31, 84

Luce, R. Duncan, 10, 12-15, 22, 40, 43, $45,49,53,55,57-62,70,72,73$ 84

Minnich, "Akos, 57

Maccheroni, Fabio, 53, 57, 61

Machina, Marth I., 28

Maksa, Gytula, 57

Marinacci, Massimo, 53, 56, 57, 61

Markowitz, Harry M, 85

Marschak, Jacob, 14.

Mendelson, Bert, 49

Merton, Robert C., 76

Minehart, Deborah., 85

Miyamoto, John, 44,55-57,84,85

Mokken, Robert J, 57

Morgenstern, Oskar, 6, 28, 54, 55, 57

Munier, Bertrand $\mathbb{R}, 36,44$

Munro, Alistain, 83,84

Nakamura, Yutaka, 29, 55, 57

Narens, Loulis, 62

Nash, John F. 98, 99, 111, 129

Neilson, Wiliam S., 85

$\mathrm{Ng}_{\text {, }}$ Yew-Kwang, 29, 83

Palfrey, Thomas R., 133

Pareto, Vilfredo, 6, 30

Payne, John W., 36, 83

Peters, Hans, 98, 121, 122, 126 
Pfanzagl, Johann, 14,53,57, 62

Pinto, Jose Luis, 36, 44, 83, 84

Plott, Charles $\mathrm{R}, 133$

Potters, Jan, 83

Pratt, Jolin W., 79, 88

Prelec, Drazen, 35, 56, 57

Quiggin, John, 35, 40, 56, 57, 75, 77, 98, 100,122

Rabin, Matthew, $6,75,76,83-85$

Raiffa, Howard, 28

Ramsey, Frank P., 27

Rhodes, Bruce, 83,84

Robinson, Richard E., 62

Robson, Arthur J., 37

Rosenthal, Robert. W., 111, 123, 126

Roth, Alvin E., 98, 99, 108, 121

Rothblum, Uriel G., 108

Rothschild, Michael, 79

Rubinstein, Ariel, 99

Safra, Zvi, 9, 10, 24, 79, 84, 98, 99

Samuelson, Paul A., 6, 8, 9, 29, 83

Santos, Tano, 81, 83, 93

Sarin, Rakesh K., 6, 55

Sattath, Shmuel, 44

Savage, Leonard J., 27-30, 35, 41, 46, 55

Schkade, David A., 36

Schmeidler, David, $6,28,29,35,40,44$, $53,55,56,79,98,99,121$

Schmidt, Ulrich, $35,54-57,80,83,85$

Schoemaker, Paul J.H, 83,84

Schwartz, Alan, 83

Scott, Dana, 14
Segal, Uzi, 56, 57

Sen, Amartya K., 6

Shafir, Eldaw, 132

Shalev, Jonathan, 83, 84, 99, 111, 123

Shapley, Lloyd S., 14, 15

Shizgal, Peter, 85

Siniscalchi, Marciano, 53,57,61.

Sipos, Jăn, 50

Skiadas, Costis, 44

Slovic, Paul, 44

Smorodinsky, Meir, 99, 103, 109, 110, 122, 123,125

Sobel, Joel, 99

Stamer, Chris, 75, 83, 84

Stigler, George J, 10

Stiglitz, Joseph E., 79

Sugden, Robert, 6, 28-31, 83,84

Sunder, Shyam, 133

Suppes, Patrick, 10, 12-15, 22, 40, 43, 45, $59-62,70,72,73$

Tallon, Jean-Marc, 79

Thaler, Richard H., 75, 76, 78, 82-85, 122

The Economist, 75

Thomson, William, 90

Tinbergen, Jan, 6

Traub, Stefan; 83,85

Twersky, Amos, $10,12-15,22,28,31,35$, $36,40,42-45,48,54,56,57,59-$ $62,70,72-75,77,78,83-87,89$ $91,96,111,122,123,132$

van Assen, Marcel A.L.M., 36, 44, 87 van Neumann, John, $6,27,28,54$ 
Varian, Hal R, 99

Vind, Karl, 61

Volij, Oscar, 99

von Neumann, John, 55,57

Wakker, Peter P., 6, 9, 13, 14, 24, 28, 29, $34,35,37,42,44,47,48,52-57$, $59-62,70-74,83-85,88-91,93$, 96,122

Werner, Jan, 28, 57

Weymark, John A., 56

Wilthien, Pierne-Henry, 76

Winet, M., 14

Winter, Eyal, 99

Yaari, Menahem. E., $56,76,79,81,82$, 98-101

Zank, Horst, 35, 44, 56, 57, 80

Zeuthen, Frederik, 29

Zhou, Lin, 99

Zilcha, Itzhak, 99

Zimnes, Joseph L., 14 


\section{Subject Index}

acceptance set, 80

act, $30,41,52$

additive separability, 9

altermative, 100,123

Archimedean axiom, 8,45

bargaining problem, 101,125

bargaining solution, 102, 125

basic utility, $78,91,124$

bounded standard sequence, 8,45

certainty equivalent, 61

CEU, 47

Choquet expected utility, 47

comoncone, 47

comonotonic, 47

comonotonic Archimedean axiom, 48

comonotonic degenerate case, 48

comonotonic preference-tradeoff consistency, 58

comonotonic strong monotonicity, 60 comonotonic tradeoff consistency, 48 complete order, 8,41

concatenation condition, 8

$\mathrm{CPT}, 50,77,90,123$

crosssover condition, 14

decision weight, 47

degenerate case, 45 difference relation, 7

difference representation, 7

disagreement alternative, 101,124

disagreement outcoune, 102

dual, 50

event, 52

expected utility, 30, 42

feasible set, 102,125

gain, $50,77,90,123$

gain acceptance set, 80

gain-loss consistency, 51

gauge, 32

global utopia point, 126

hexagon condition, 9

individual monotonicity, 110

intrinsic value, 132

Kalai-Rosenthal solution, 126

Kalaj-Smorodinsky solution, 103,125

loss, $50,77,90,123$

loss acceptance set: 80

losis aversion index, 78,124

lottery, 77, 90, 100, 123

midpoint condition, 11 
mixwed lotitey, 80,91

neutrality, 8

nominal value, 132

null/nonnull state, 42

order-dense set, 45

outcome, $7,77,90$

Pareto optimal set, 102, 125

preference relation, 7,41

preference-tradeoff consistency, 58

probabilistic risk aversion, 101

prospect theory, $50,77,90,123$

pure lottery, 91

rank-dependent utility, 54,100

rank-ordering permutation, 47

RDU, 100

reference point, $50,77,90,123$

relation $\sim \sim_{c}^{*}, 48$

relation $\sim \sim_{F}^{*}, 42$

relation $\sim \sim_{s c}^{*}, 51$

relation $\sim *, 32$

representing function, $7,41,77,90,123$

reversal condition, 10

scale invarience, 110

SEU, 42

SEU-set, 42

sigrilıcomoncone, 50

sign-comonotonic, 51

sigrt-comonotonic preference-tradeoff consistency, 59

sigr-comonotonic strong monotonicity, 60 sign-comonotonic tradeoff consistency, 51 solvabillity, 8,45

si-concatenation condition, 11

standard sequence, 8,44

standard-sequence condition 10

state space, 41,52

strong concatenation condition, 12

strong crossover condition, 14

strong indifference-tradeoff consistency, 64

strong monotonicity, 60

strong teversal condition, 12

subjective expected utility, 30,42

symmetry, 109

Thomsen condition, 14

tradeoff consistency, 33, 43, 46

trivial act, 42

truly mixed, 51

utility function, $7,42,47,50,77,90,100$, 123

utility risk aversion, 101

utopia point, 103, 125

weak monotonicity, 41

weak order, 8,41

weak Pareto optimality, 110

weak separability, 8

weighting function, $47,77,90,100,123$ 


\section{Summary}

This thesis is a collection of essay on decision making. The first of these essays (Chapter 2) deals with the traditional and controversial topic of ordinal versus cardinal utility. By providing simpler and more general preference foundations for difference representations, the chapter shows that cardinal utility is easier to obtain than traditionally thought. The chapter unifies all earlier derivations by showing that they can be obtained as direct corollaries.

Chapter 3 introduces a new preference condition that can be used to justify (or criticize) expected utility. The approach of the chapter is alternative to Savage"s, and is accessible to readers without a mathematical background. It is based on a mothod for deriving "comparisons of tradeoffs" from ordinal preferences. "The new condition simplifies previously-published tradeoff conditions, and at the same time provides more general and more powerful tools to specialists. The condition is more closely related to empirical methods for measuring utility than its predecessors. It provides a unifying tool for quantitatively measuring, qualitatively testing, and normatively justifying expected utility.

Chapter 4 extends the methods from Chapter 3 to rank-dependent utility and prospect theory, and thereby gives axiomatic foundations for those decision models. The resulting foundations are, at a time, more general and more accessible than earlier results. The conditions used are better suited for empirical measurements of utility than earlier conditions, and accordingly are easier to test. Whereas Chapter 3 is particularly addressed to readers without a strong mathematical background, Chapter 4 can be seen as the theoretical, more advanced, and more general counterpart.

Chapters 5 and 6 deal with loss aversion in prospect theory. To a considerable extent, risk aversion as it is commonly observed is caused by loss aversion. Chapters 5 proposes a quantitative index of loss aversion. Under prospect theory, the proposal leacis to a decomposition of risk attitude into three distinct components: intrinsic utility, probability 
weighting, and loss aversion. The main theorem shows how the index of loss aversion of different decision makers can be compared through observed choices. Chelpter 6 points out the difficulties in quantifying loss aversion for general situations with nommonetary outcomes. A theorem shows bow, nevertheless, also for general outcomes it is possible to obtain relative comparisons of loss aversion.

Chapters 7 and 8 apply rank-dependent utility and prospect theory to bargaining garne theory. Under rank-dependent utility two factors infuence the risk attitude of a decision maker: the utility function and the probability weighting function. Arising from the same definition of risk aversion, two forms of risk aversion can be distinguished: utility risk aversion and probabilistic risk aversion. The main finding of Chapters 7 is that these two forms of risk aversion can have surprisingly opposite consequences for bargaining solutions that exhibit a weak monotonicity property. In particular, in a large class of bargaining problems both increased utility risk aversion and decreased probabilistic rjsk aversion of the opponent are advantageous for a player. Chapter 8 examines the influence of loss aversion in bargaining problems under prospect theory. It is shown that it is usually better for a player if the opponent is less loss averse. These results hold in particular for the KalaiSmorodinsky and the Kalai-Rosenthal bargaining solutions. The Nash bargaining solution does not behave regularly in this respect.

Chapter 9 is about an empirical experiment. The change of currency in Europe gave a unique opportunity to test whether people, when evaluating money, think in terms of numbers instead of in terms of real values. It is hard to disentangle the influence of numbers and real values on risk attitude. The currency conversion gave the opportunity to keep one of the two aspects fixed while the other varies, and to observe changes in risk attitude caused by changing numbers separately from that caused by changing real values. In Belgium the scale changed by a, considerable, factor 40 . Therefore, we chose Belgium to carry out an empirical study to test the effects of value versus mumerical changes. We measured the risk attitudes of 87 subjects in December 2001, shortly before the introduction of the Euro, and did the same for 92 subjects in May 2002, when people started to get accustomed to the Euro. We find that changes in value while keeping numbers constant do affect risk attitude, but changes in numbers while keeping values constant do not, in agreement with postulates of rationality. 


\section{About the Author}

Veronila Köbberling was born on September 13, 1976 in Göttingen, Germany. From October 1995 till November 1998 she studied mathematics at the University of Technology Aachen, Germany, and at the Queen Mary and Westfeld College London, England. In November 1998 she received the degree, Master of Science in Mathematics, from the University of London. Since April 2001 she also studies applied computer science at the University of Hagen, Germany.

From May 1999 till April 2003, Veronika Köbberling was a Ph.D.-student at the Department of Quantitative Economics at Maastricht University, The Netherlands. Her research on decision making and game theory was supervised by Prof. Dr. Hans Peters and Prof. Dr. Peter P. Wakker. In spring 2002 she has spent two month as a visiting scholar at the Kellogg School of Management, Northwestern University, Evanston, USA. 